\title{
Cellular function and toxicity of the Parkinson's disease-related genes a-synuclein and catp-6 in C. elegans
}

\section{PhD Thesis}

In partial fulfilment of the requirements

for the degree „Dr. rer. nat.”

in the Neuroscience Program

at the Georg August University Göttingen,

Faculty of Biology

submitted by

Nora Wender

born in

Elmshorn, Germany 
I hereby declare that the $\mathrm{PhD}$ thesis entitled "Cellular function and toxicity of the Parkinson's disease-related genes $\alpha$-synuclein and catp- 6 in C. elegans" was written independently and with no other sources and aids than quoted.

Göttingen, $13^{\text {th }}$ March, 2012

Nora Wender

Thesis Committee Members:

Dr. Stefan Eimer

European Neuroscience Institute

Prof. Dr. Reinhard Jahn

Max Planck Institute for Biophysical Chemistry

Prof. Dr. Andreas Wodarz

Georg August University Göttingen 
To my parents 


\section{Table of contents}

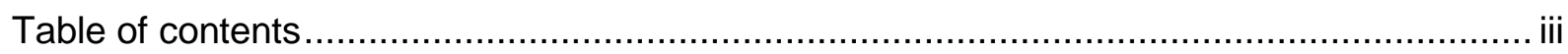

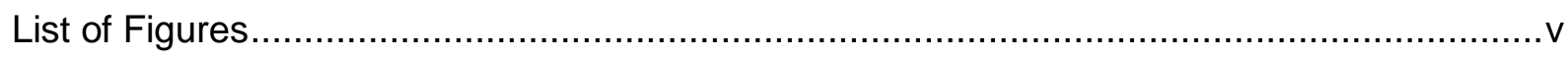

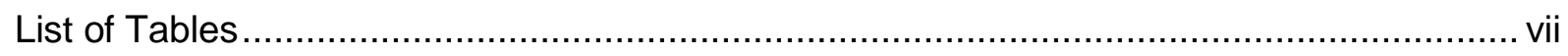

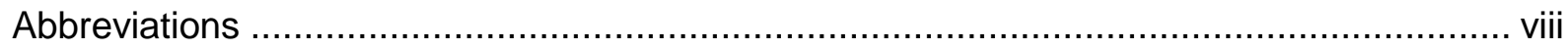

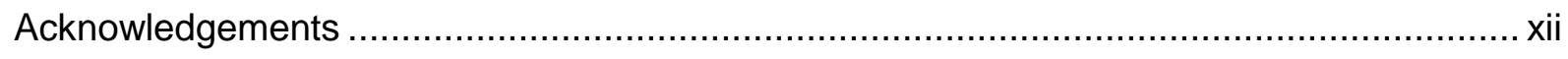

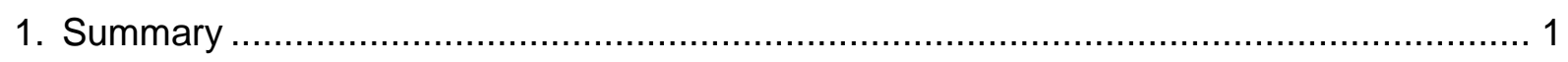

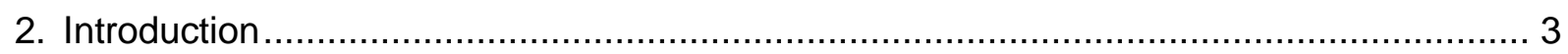

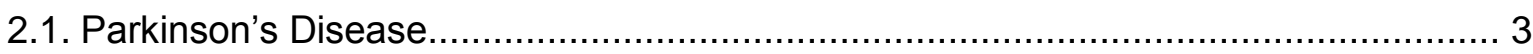

2.1.1. Particular vulnerability of DA neurons in the SN .................................. 4

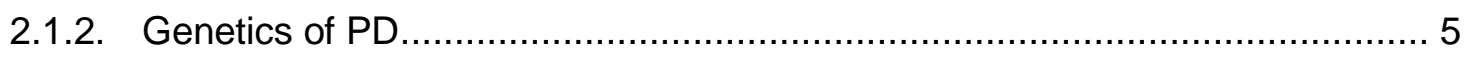

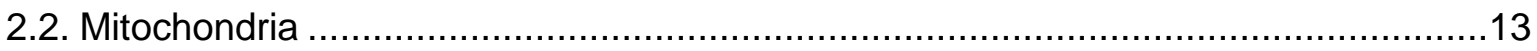

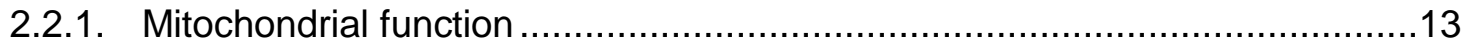

2.2.2. Mitochondrial dynamics ............................................................. 14

2.2.3. Mitochondrial quality control - stress response pathways and

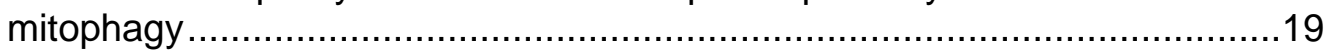

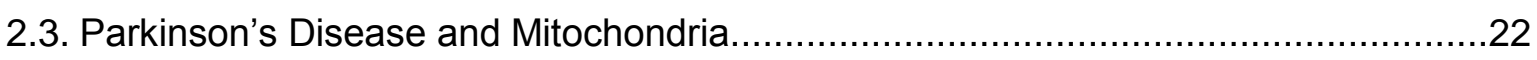

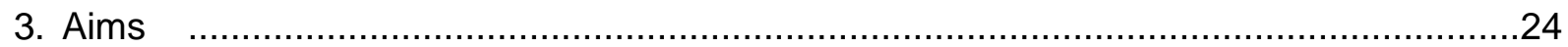

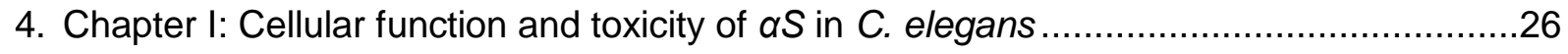

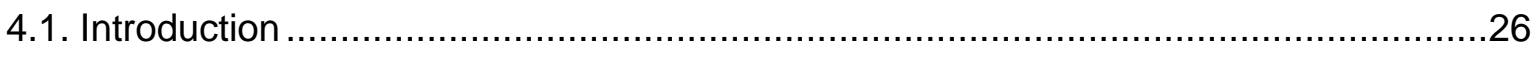

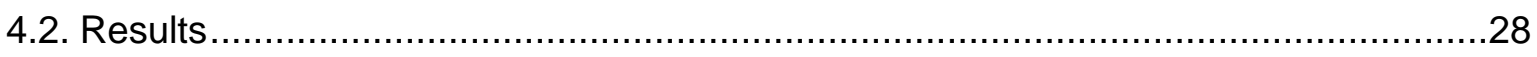

4.2.1. Generation of synthetic aS variants that are not forming fibrils in vitro ..........28

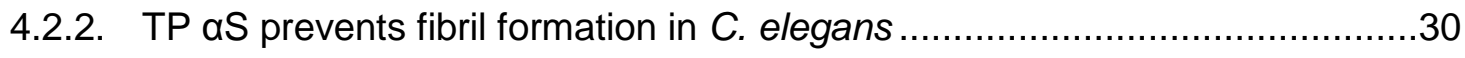

4.2.3. TP aS exhibits increased neurotoxicity in $C$. elegans.................................31

4.2.4. Expression of $\alpha S$ leads to drastic changes of mitochondrial morphology in $C$. elegans muscle cells

4.2.5. Mitochondrial fragmentation similar to that seen upon expression

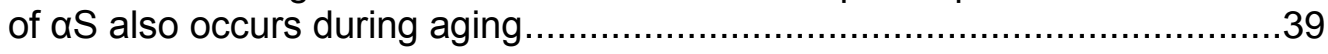

4.2.6. Changes in mitochondrial morphology caused by expression of $\alpha S$ resemble those seen under starvation...

4.2.7. Fibril formation and mitochondrial fragmentation can also be seen in $C$. elegans neurons expressing aS .........................................4

4.3. Discussion

4.3.1. Proline mutations in designed variants of $\alpha S$ prevent fibril formation in vitro and in vivo

4.3.2. TP aS exhibits increased neurotoxicity in $C$. elegans.................................45

4.3.3. Expression of $\alpha S$ leads to drastic changes of mitochondrial morphology in $C$. elegans muscle cells and neurons 
5. Chapter II: Cellular function and toxicity of catp-6 (PARK9) in C. elegans.......................58

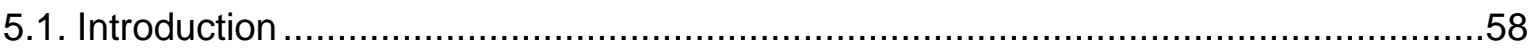

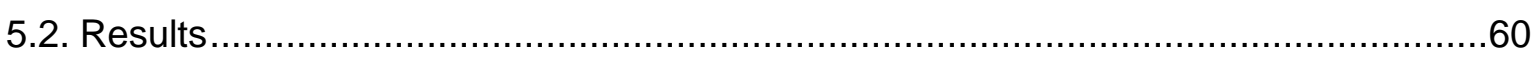

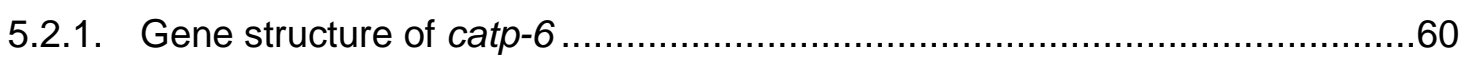

5.2.2. Deletion of catp-6 leads to egg laying defects, causing a reduction in brood size......................................................................60

5.2.3. Postembryonic development is delayed in catp-6 ................................62

5.2.4. catp-6 mutants display locomotion defects due to muscular

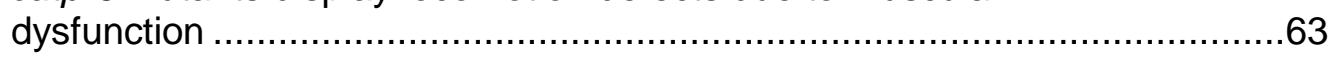

5.2.5. catp- 6 is expressed in multiple tissues ....................................................64

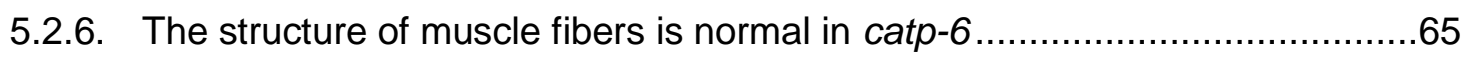

5.2.7. catp- 6 mutants show vulva muscle defects ..............................................66

5.2.8. Mitochondrial morphology is severely affected in muscle cells

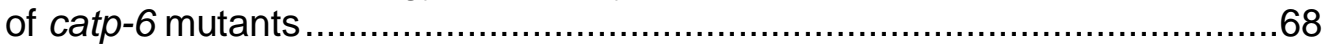

5.2.9. Mitochondrial morphology is already affected at larval stages

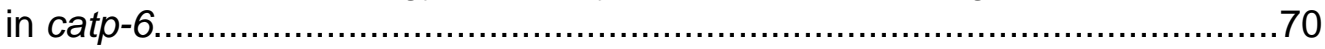

5.2.10. EM analysis reveals collapsing of mitochondria and loss of mitochondrial cristae in catp- 6 mutants

5.2.11. The mitochondrial fission protein DRP-1 is clustering in close proximity to the nucleus in BWM cells of catp-6 mutants ...........................72

5.2.12. RNAi against mitochondrial dynamics genes ............................................ 75

5.2.13. Changes in mitochondrial morphology in neurons of catp-6 worms...............77

5.2.14. Deletion of catp- 6 leads to impaired mitochondrial function...........................79

5.2.15. The mitochondrial stress response is induced in catp- 6 mutants...................82

5.2.16. catp-6 mutants exhibit increased sensitivity to oxidative stress and a reduced life span

5.2.17. Increased activation of AMPK and induction of autophagy in catp- 6 deletion mutants

5.2.18. No excessive apoptosis in the germline of catp-6 ....................................90

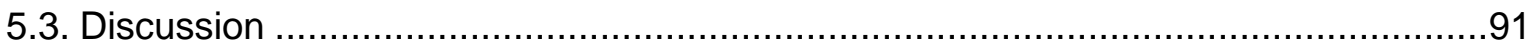

5.3.1. Multiple factors contribute to the reduced brood size of catp-6 mutants

5.3.2. Reduced locomotion of catp- 6 is based on muscular defects that are already apparent at larval stages .............................................95

5.3.3. Deletion of catp-6 leads to changes in mitochondrial morphology .................96

5.3.4. Impaired mitochondrial function in catp-6 deletion mutants ........................100

5.3.5. catp- 6 mutants show an increased sensitivity towards oxidative stress, activation of the mitochondrial stress response and a reduced lifespan ......103

5.3.6. Increased activation of AMPK is not accompanied by increased basal autophagy 105

5.3.7. Deletion of the lysosomal P-type ATPase CATP-6 causes mitochondrial defects - novel link between lysosomes and mitochondria

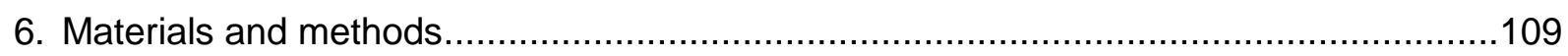

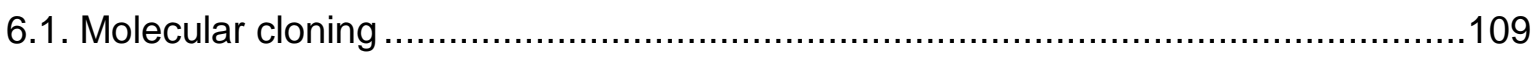

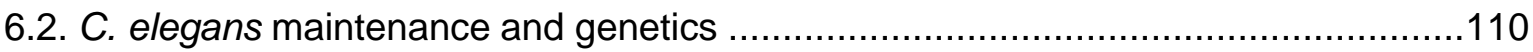

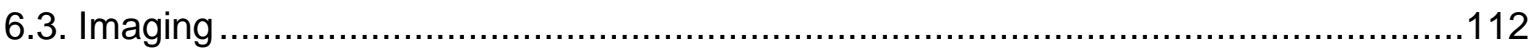




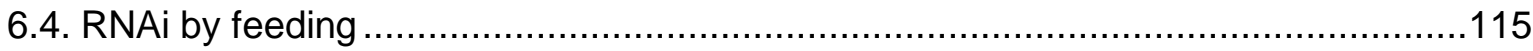

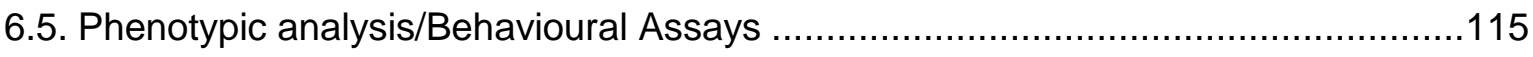

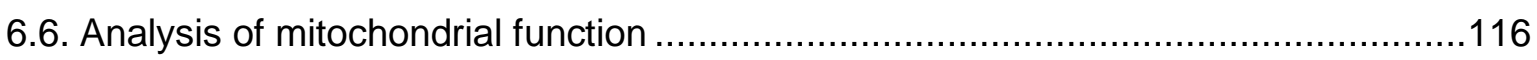

6.7. Assessing induction of the cellular stress responses..........................................117

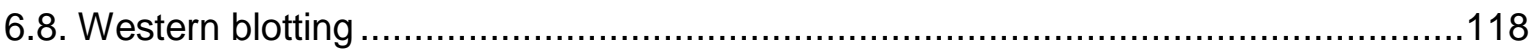

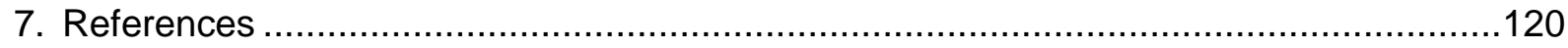

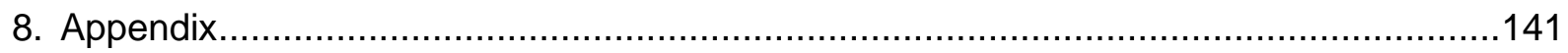

8.1. Alignment of $C$. elegans CATP-6 and human ATP13A2 ..................................141

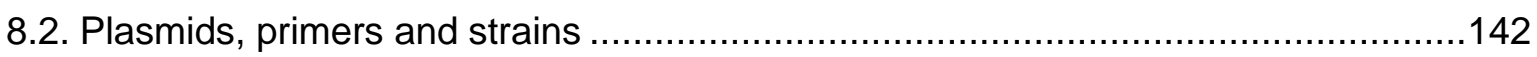

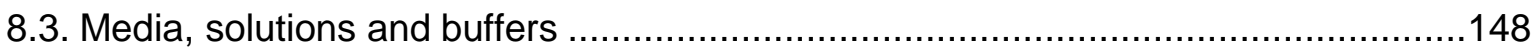

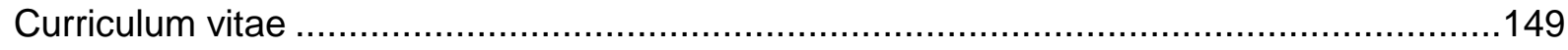

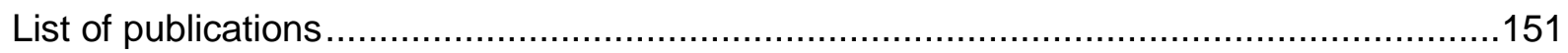




\section{List of Figures}

Fig. 2.1: Lewy bodies are the histological hallmarks of PD .......................................... 3

Fig. 2.2: Structure and reaction cycle of P-type ATPases............................................. 9

Fig. 2.3: Pathways involved in PD pathogenesis and PD-associated genes affecting

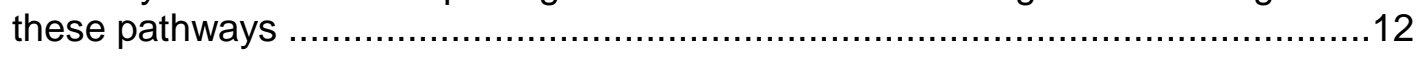

Fig. 2.4: Mitochondrial electron transfer chain ........................................................14

Fig. 2.5: Fusion of the mitochondrial outer membranes is mediated by mitofusins in mammals.

Fig. 2.6: OPA1 mediates inner membrane fusion in mammals ....................................16

Fig. 2.7: Mitochondrial fission is mediated by Drp1 ..................................................... 16

Fig. 2.8: Phosphorylation status of Drp1 regulates mitochondrial fission activity.................17

Fig. 2.9: Mitochondrial unfolded protein response $\left(U^{2} R^{m t}\right)$ in $C$. elegans ........................20

Fig. 2.10: Removal of damaged mitochondria via mitophagy ........................................21

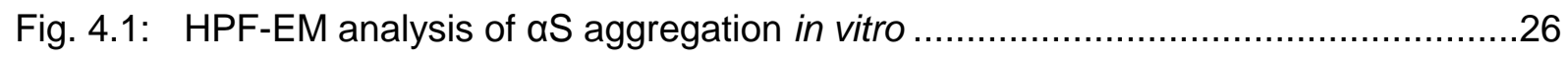

Fig. 4.2: Synthetic variants of $\alpha S$ exhibit decreased rates of fibril formation in vitro because of impaired $\beta$-sheet structures.

Fig. 4.3: Formation of fibrillar aggregates is absent in C. elegans muscle cells expressing TP aS....

Fig. 4.4: Neurotoxicity is increased in the pre-fibrillar variant TP aS

Fig. 4.5: Changes in mitochondrial morphology in $C$. elegans muscle cells expressing aS.

Fig. 4.6: $E M$ analysis of changes in mitochondrial morphology in $C$. elegans muscle cells induced by expression of wt $\alpha \mathrm{S}$

Fig. 4.7: Effect of different $\alpha S$ variants on mitochondrial morphology in C. elegans BWM cells

Fig. 4.8: Mitochondrial fragmentation in C. elegans muscle cells during aging

Fig. 4.9: Mitochondrial fragmentation in BWM cells upon starvation of wild type worms

Fig. 4.10: Fibril formation and mitochondrial fragmentation in $C$. elegans neurons expressing wt aS

Fig. 5.1: Gene structure of catp- 6 and position of deletions

Fig. 5.2: catp-6 deletion mutants have an egg-laying defect and protruding vulva phenotype

Fig. 5.3: Postembryonic development is delayed in catp-6 deletion mutants

Fig. 5.4: catp- 6 worms have a movement defect which can be rescued by expression of catp- 6 in muscle cells and which is already apparent at larval stages.

Fig. 5.5: catp-6 expression pattern

Fig. 5.6: Myofilament organization in BWM cells is not affected in catp-6 deletion mutants

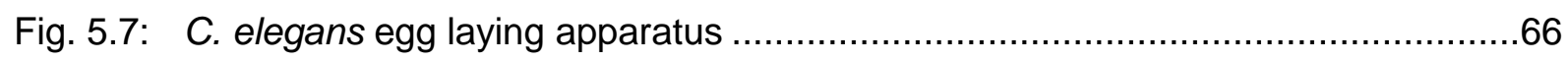

Fig. 5.8: Vulva muscle defects in catp-6 deletion mutants 
Fig. 5.9: Mitochondrial elongation and perinuclear clustering in catp- 6 deletion mutants

Fig. 5.10: Changes in mitochondrial morphology and perinuclear clustering are already present in L3 larvae of catp-6 mutants

Fig. 5.11: EM analysis of mitochondria in BWMs of catp-6 mutants

Fig. 5.12: Changes in mitochondrial morphology and distribution of the mitochondrial fission protein DRP-1 in muscle cells of catp- 6 mutants.

Fig. 5.13: Effect of silencing of mitochondrial dynamics genes on mitochondrial morphology in wild type worms and catp-6 mutants.

Fig. 5.14: Changes in mitochondrial morphology in neurons of catp- 6 mutants ...................78

Fig. 5.15: Mitochondrial membrane potential is reduced in catp- 6 mutants 79

Fig. 5.16: Composition of complex IV of the respiratory chain is changed in catp- 6 mutants.

Fig. 5.17: Increased oxygen consumption in catp-6 mutants.

Fig. 5.18: Mitochondrial stress response is specifically activated in deletion mutants of catp-6.

Fig. 5.19: Several tissues of catp- 6 mutants show a strong induction of the mitochondrial stress response

Fig. 5.20: catp-6 mutants show increased sensitivity to paraquat and a reduced life span

Fig. 5.21: Increased phosphorylation of AMPK in catp-6 mutants.

Fig. 5.22: Increased induction of autophagy in eggs of catp- 6 mutants .88

Fig. 5.23: Deletion of catp-6 causes egg defects. .89

Fig. 5.24: No increase in germline apoptosis in catp-6 mutants. .90

Fig. 5.25: Formation of the vulva-uterine connection in C. elegans .92

\section{List of Tables}

Table 2.1: Overview of PD-associated genes. 6

Table 5.1: Mutations in catp-6 cause Egl and pVul defects and reduced brood size.

Table 5.2: Vulva muscle defects in catp-6 .68

Table 8.1: List of plasmids used in this study. 142

Table 8.2: Cloning strategies for constructs used in this study 143

Table 8.3: List of primers used in this study 144

Table 8.4: List of injection mixes used in this study 146

Table 8.5: List of strains used in this study 146 


\section{Abbreviations}

aa

$\mathrm{AB}$

$A C$

ADE

ADP

AEU

AMP

Amp

AMPK

Asp

ATG1

ATG8

ATM

ATP

ATP13A2

$\mathrm{BH} 3$

BN-PAGE

BWM

Caf4

cAMP

$\mathrm{CC}$

CCCP

CCR4

cDNA

CEP

CFP

CGC

COMT

COR

$\mathrm{CSPa}$

cyt c

DA

dd $\mathrm{H}_{2} \mathrm{O}$

DIC

DiSC3(5)
Amino acid

Antibody

Anchor cell

Anterior deirid

Adenosine diphosphate

Arbitrary expression unit

Adenosine monophosphate

Ampicillin

AMP-activated kinase

Aspartate

Autophagy related 1

Autophagy related 8

Ataxia telangiectasia mutated

Adenosine triphosphate

ATPase type 13A2

$\mathrm{Bcl}-2$ homology domain 3

Blue native polyacrylamide gel electrophoresis

Body wall muscle

CCR4 associated factor 1

Cyclic AMP

Coelomocyte

Carbonyl cyanide m-chlorophenyl hydrazone

Chemokine (C-C motif) receptor 4

Complementary DNA

Cephalic

Cyan fluorescent protein

Caenorhabditis Genetics Center

Catechol-O-methyl-transferase

C-terminal of ROC

Cystein string protein alpha

Cytochrome c

Dopamin/Dopaminergic

Double-destilled water

Differential interference contrast

3,3' - Dipropylthiadicarbocyanine iodide 


\begin{tabular}{|c|c|}
\hline DN & Dominant negative \\
\hline DNA & Deoxyribonucleic acid \\
\hline Drp1/DRP-1 & Dynamin-related protein 1 \\
\hline EGFP & Enhanced GFP \\
\hline Egl & Egg laying defective \\
\hline EM & Electron microscopy \\
\hline ER & Endoplasmic reticulum \\
\hline ETC & Electron transfer chain \\
\hline FBXO7 & F-box protein 7 \\
\hline FCCP & Carbonyl cyanide 4-(trifluorpmethoxy)-phenylhydrazone \\
\hline Fis $1 / F I S-1$ & Fission 1 \\
\hline GABARAP & Gamma-aminobutyric acid receptor associated protein \\
\hline GATE-16 & Golgi-associated ATPase enhancer of $16 \mathrm{kDa}$ \\
\hline GED & GTPase effector domain \\
\hline GFP & Green fluorescent protein \\
\hline GIGYF2 & GRB10 interacting GYF protein \\
\hline GRB10 & Growth factor receptor-bound protein 10 \\
\hline GSH & Glutathion \\
\hline GTP & Guanosine triphosphate \\
\hline H2-DCF-DA & 2,7-dichlorodihydrofluorescein-diacetate \\
\hline HSN & Hermaphrodite specific neuron \\
\hline HPF & High pressure freeze \\
\hline HSP & Heat shock protein \\
\hline $\mathrm{HtrA2}$ & High temperature requirement protein $\mathrm{A} 2$ \\
\hline IMM & Inner mitochondrial matrix \\
\hline IMS & Inner membrane space \\
\hline IPTG & Isopropyl $\beta$-D-thiogalactopyranoside \\
\hline $\mathrm{kb}$ & Kilobase \\
\hline $\mathrm{KCN}$ & Potassium cyanide \\
\hline KD & Knockdown \\
\hline KO & Knockout \\
\hline KRS & Kufor-Rakeb syndrome \\
\hline LB & Lewy body; In Materials and methods section: Luria Bertani \\
\hline LC & Locus coerulius \\
\hline LC3 & Microtubule-associated proteins 1A/1B light chain 3 \\
\hline L-DOPA & L-3,4-dihydroxyphenylalanine \\
\hline LGG-1 & LC3, GABARAP and GATE-16 family 1 \\
\hline
\end{tabular}




\begin{tabular}{|c|c|}
\hline LKB1 & Liver kinase B1 \\
\hline LRR & Leucine-rich repeat \\
\hline LRRK2 & Leucine-rich repeat kinase 2 \\
\hline MAO-B & Monoamine oxidase B \\
\hline Mdv1 & Mitochondrial division 1 \\
\hline Mfn1/Mfn2 & Mitofusin 1/Mitofusin 2 \\
\hline MPP+ & 1-methyl-4-phenylpyridinium \\
\hline MPTP & 1-methyl-4-phenyl-1,2,3,6-tetrahydropyridine \\
\hline mRFP & Monomeric RFP \\
\hline mTOR & Mammalian target of Rapamycin \\
\hline mTORC1 & mTOR complex 1 \\
\hline $\mathrm{NADH}$ & Nicotinamide adenine dinucleotide \\
\hline $\mathrm{NaN}_{3}$ & Sodium azide \\
\hline NCAP & Non-amyloid beta-component of plaques \\
\hline NGM & Nematode growth medium \\
\hline NMR & Nuclear magnetic resonance \\
\hline OCR & Oxygen consumption rate \\
\hline OMM & Outer mitochondrial matrix \\
\hline Opa1 & Optic atrophy 1 \\
\hline OVN & Overnight \\
\hline PAGE & Polyacrylamide gel electrophoresis \\
\hline PALM & Photo-activated localization microscopy \\
\hline PCR & Polymerase chain reaction \\
\hline PD & Parkinson's disease \\
\hline PE & Phosphatidyl ethanolamine \\
\hline PED & Postembryonic development \\
\hline PGC-1a & Peroxisome proliferator-activated receptor gamma coactivator 1 -alpha \\
\hline PINK1 & PTEN-induced putative kinase 1 \\
\hline PKA & Protein kinase $A$ \\
\hline PLA2G6 & Phospholipase A2 group VI \\
\hline PTEN & Phosphatase and tensin homolog \\
\hline pVul & Protruding vulva \\
\hline RFP & Red fluorescent protein \\
\hline RNA & Ribonucleic acid \\
\hline RNAi & RNA interference \\
\hline ROC & Ras of complex proteins \\
\hline ROCO & $\mathrm{ROC} / \mathrm{COR}$ \\
\hline
\end{tabular}




\begin{tabular}{|c|c|}
\hline ROS & Reactive oxygen species \\
\hline rpm & Rotations per minute \\
\hline SD & Standard deviation \\
\hline SDS-PAGE & Sodium dodecyl sulfate polyacrylamide gel electrophoresis \\
\hline SEM & Standard error of the mean \\
\hline Ser & Serine \\
\hline siRNA & Small interfering RNA \\
\hline SM & Sex myoblast \\
\hline SN & Substantia nigra \\
\hline SNCA & Synuclein alpha \\
\hline SOD & Superoxide dismutase \\
\hline STED & Stimulated Emission Depletion \\
\hline STORM & Stochastic Optical Reconstruction Microscopy \\
\hline Thr & Threonine \\
\hline TOM70 & Translocase of outer mitochondrial membrane 70 \\
\hline TM & Transmembrane \\
\hline TP & Triple proline \\
\hline $\mathrm{UCH}-\mathrm{L} 1$ & Ubiquitin carboxyl-terminal esterase L1 \\
\hline ULK1 & Unc-51-like kinase 1 \\
\hline UPR & Unfolded protein response \\
\hline $\mathrm{UPR}^{\mathrm{ER}}$ & Endoplasmic Reticulum unfolded protein response \\
\hline UPR $^{\text {cyt }}$ & Cytosolic unfolded protein response \\
\hline $\mathrm{UPR}^{\mathrm{mt}}$ & Mitochondrial unfolded protein response \\
\hline UPS & Ubiquitin proteasome system \\
\hline Utse & Uterine seam cell \\
\hline VC & Ventral cord \\
\hline VDAC1 & Voltage-dependent anion channel \\
\hline VNC & Ventral nerve cord \\
\hline VPC & Vulva precursor cell \\
\hline VU & Ventral uterine precursor cell \\
\hline wt & Wild type \\
\hline X-Gal & 5-bromo-4-chloro-3-indolyl-beta-D-galacto-pyranoside \\
\hline YFP & Yellow fluorescent protein \\
\hline aS & a-synuclein \\
\hline$\beta S$ & $\beta$-synuclein \\
\hline$\Delta \Psi_{\mathrm{m}}$ & Mitochondrial membrane potential \\
\hline
\end{tabular}




\section{Acknowledgements}

The last years, in which I was working on this thesis, were a very exciting and intense period of my life and I want to thank a number of persons who played an important role during this time and were supporting me on a professional or personal level.

First I would like to thank my supervisor Dr. Stefan Eimer for giving me the opportunity to work in his lab, for sharing his knowledge and introducing me to the field of $C$. elegans neurogenetics. I am thankful for his supervision and for his enthusiasm and fascination for science.

I would like to express my gratitude to my thesis committee members Prof. Dr. Reinhard Jahn and Prof. Dr. Andreas Wodarz for their interest, advice and suggestions during the thesis committee meetings which were of great support for this project. Furthermore, I would like to thank our collaborators, Prof. Dr. Christian Griesinger and Prof. Dr. Markus Zweckstetter for collaboration in the study of aS aggregation and toxicity, Prof. Dr. Christian Haass and Dr. Nicole Exner for collaboration in the investigation of aS-induced changes in mitochondrial morphology as well as Prof. Dr. Peter Rehling and Dr. Jan Dudek for isolation and functional analysis of mitochondria for the catp- 6 project.

The International Max Planck Research School (IMPRS) for Neurosciences and the Göttingen Graduate School for Neurosciences and Molecular Biology (GGNB) were providing an excellent environment for my research and I would like to thank Prof. Dr. Michael Hörner, Sandra Drube, Dr. Steffen Burkhardt and Kirsten Pöhlker for coordination of these programs and for their assistance throughout my PhD studies. Furthermore, I would like to acknowledge the Dorothea Schlözer Program of the University of Göttingen for financial support and the German National Academic Foundation ("Studienstiftung des deutschen Volkes") for giving me the opportunity to take part in very inspiring seminars and summer schools.

I am also thankful to Christiane Becker and Dr. Synnöve Beckh in the administration and coordination office of the European Neuroscience Institute for administrative support. Thanks to Ali Pilehwar and Matthias Weyl for help regarding IT questions, to Heiko Röhse for help with the confocal and spinning disk microscopes and to Frank Kötting and Magda Krause for practical support.

I would like to thank all members of the Eimer lab for creating such a nice working environment, for help and support. Specials thanks to Dr. Jan Hegermann for Electron microscopy and to Dr. Sabine König and Katrin Schwarze for their help with cloning, crosses and experiments. I would also like to acknowledge Victor Bustos, Robin Kemmler, David Vasmer and Ahmed Abdelsamad for help during their lab rotations. I am especially thankful to Dr. Ling Luo who was my supervisor during the lab rotation and also later on, when I started my 
PhD project, was always supporting me and sharing his knowledge and experience. Many thanks to Mandy Hannemann and Dr. Nikhil Sasidharan for stimulating discussions, help and advice and wonderful lunch breaks. Special thanks to Mandy for introducing me to the world of Loriot and for encouraging me to join for the gym - at least sometimes successfully. I would also like to thank the other members of the lab Dr. Marija Sumakovic, Maike Kittelmann, Janny Suekyoung Jeon, Dr. Christian Olendrowitz and Dr. Christoph Wrede - it was really a pleasure working with you!

Moreover, I am thankful to my classmates of the Neuroscience and Molecular Biology programs, particularly to Andreas Schindler for his friendship, music suggestions and in general for the wonderful time that we spend together here in Göttingen during the Master's studies. I also thank Dr. Annette Denker, Frederik Köpper, Cornelius Schneider and Broder Schmidt for nice wine seminars, dinners and for very special trips to the North Sea.

Furthermore, I am very thankful to Felicitas Sedlmair, Dr. Thomas Schultze, Dr. Johannes Schmidt-Hieber, Frederik Köpper, Lars Kasper, Dr. Nadira Faulmüller as well as Jakob and Katharina Bierwagen for joint dinners and gaming nights and "Big Bang Theory" sessions.

Special thanks to Felicitas Sedlmair for introducing me to the world of Tango Argentino and to Dorothea Stracke for joint tap dance adventures.

I am particularly thankful to Alexandra Diewald, Janina Rothkamp and Johannes Hensler for the long-lasting friendship, for always being there for me, especially in times when things were not working out in the way I expected, and simply for the great time that we spent together.

Finally, I would like to thank my parents, Doris Altmeyer-Wender and Dr. Herbert Wender for their constant love and support. I am very thankful that you were always standing behind and were encouraging me to ensue my plans. 


\section{Summary}

Parkinson's disease (PD) is the second most common neurodegenerative disease in industrialized countries. However, the molecular mechanisms leading to neuronal death in PD still remain unclear. About $5-10 \%$ of PD patients suffer from familial forms of the disease and so far, 16 chromosomal regions have been associated with familial PD. As disease progression and outcome of familial and sporadic forms of the disease are very similar, studying the cellular function of genes associated with familial PD might hopefully yield in a better understanding of the molecular pathways that are involved in the disease pathogenesis. Two major pathways have been proposed to be causing neuronal cell death in PD: dysfunction of the cellular protein degradation machineries and accumulation of misfolded proteins as well as mitochondrial dysfunction. The aim of the present study was to investigate the cellular function and toxicity of two PD-associated genes, $\alpha$-synuclein ( $\alpha S) / P A R K 1$ and ATP13A2/PARK9, in the nematode C. elegans.

Protein aggregations in the brain of PD patients, the so-called "Lewy bodies", are one of the main pathological hallmarks in PD. The main component of Lewy bodies is the protein aS. Mutations, as well as increased expression levels of $\alpha S$, have been shown to cause familial PD. In both cases increased aggregation rates of aS have been demonstrated. Therefore, until now it was believed that fibrillar $\alpha S$ aggregates cause neurodegeneration in PD. It remained unclear however, whether insoluble fibrillar aggregates of $\alpha \mathrm{S}$ or rather smaller oligomeric precursors are causing toxicity. To address this question we decided to compare the aggregation properties and toxicity of different variants of aS including synthetic variants that do not form fibrillar aggregates but stop aggregation at the stage of small soluble oligomers. This analysis revealed that the oligomer-promoting variants exhibit higher toxicity than wild type (wt) aS or the PD mutants. This suggests that not the fibrils but rather the soluble oligomers are the toxic species of aS. However, it remained unresolved which molecular mechanisms are involved in mediating aS toxicity. To shed light on this aspect, the effects of $\alpha S$ on mitochondrial morphology were investigated. It was previously reported that aS can localize to mitochondria and that overexpression can cause mitochondrial pathology. Electron microscopy (EM) and spinning disk confocal microscopy analysis demonstrated that mitochondrial morphology is severely affected in both C. elegans muscle cells and neurons expressing aS. While expression of wt human aS in muscle cells induces both mitochondrial fragmentation and the occurrence of long and thin mitochondrial tubules, expression of the oligomer-promoting variant of $\alpha \mathrm{S}$, for which increased toxicity was demonstrated, predominantly leads to long, thin and interconnected mitochondria. Based on these observations we propose a model in which the effects of aS expression on mitochondrial morphology are dependent on the relative abundance of different species of aS (monomeric vs. different oligo- 
meric forms and fibrils) and their relative amounts. The finding that mitochondrial fragmentation is also occurring in aged worms without expression of aS suggests that expression of $\alpha \mathrm{S}$ accelerates the physiological aging process.

The second part of my $\mathrm{PhD}$ thesis is concerned with the cellular function and toxicity of another PD-related gene, the lysosomal P-type ATPase catp-6, which is the C. elegans ortholog of human ATP13A2 (PARK9). Phenotypic analysis of deletion mutants of this gene revealed severely impaired egg laying which causes a strong reduction in brood size. The mutants also exhibit a markedly delayed postembryonic development. Thus, asynchronous development of different tissues might be causing the defects in the egg laying apparatus as coordinated differentiation is crucial for establishing the connection of the different tissues involved in egg laying. Furthermore, a locomotion defect, which is already apparent at larval stages and can be rescued by restoring CATP-6 function specifically in muscle cells, was detected in catp- 6 mutants. This raised the question what causes these defects on a cellular level. As a functional interaction of CATP- 6 and $\alpha S$ was reported previously and given that aS causes drastic reorganization of the mitochondrial network, it was decided to investigate mitochondrial morphology and function in deletion mutants of catp-6. Loss of function of CATP-6 leads to severe changes in mitochondrial morphology with formation of networks of long and very thin mitochondrial tubules and clustering of mitochondria around the nucleus. The observations that the mitochondrial fission protein DRP-1 forms large clusters in catp- 6 mutants and that RNAi against $d r p-1$ did not change mitochondrial morphology in catp- 6 mutants suggest that loss of function of DRP-1 might be the cause for reorganization of the mitochondrial network in catp-6 mutants. Besides morphological defects, also functional impairments of the mitochondria were observed upon loss of function of CATP-6, with deletion mutants exhibiting a reduced membrane potential, changes in the composition of complex IV as well as increased oxygen consumption. A possible explanation for the latter might be an up-regulation of mitochondrial mass. catp- 6 mutants moreover exhibited increased sensitivity to oxidative stress and a reduced lifespan. In agreement with this, the mitochondrial stress response was found to be strongly up-regulated in catp-6 mutants. Future investigations are needed to determine whether these effects could be due to excess reactive oxygen species (ROS) production by the mutants caused by defects in the respiratory chain. In line with mitochondrial function being compromised in the mutants, we see an increased activation of the cellular energy sensor AMPK, which, in reaction to a rise in the cellular AMP/ATP ratio, acts to inhibit anabolic process and stimulate catabolic processes in the cell in order to maintain the cellular energy balance. In future studies it will be important to determine the exact molecular and cellular mechanisms how loss of function of the lysosomal P-type ATPase CATP-6 can cause such drastic effects on mitochondrial morphology. 


\section{Introduction}

\subsection{Parkinson's Disease}

Parkinson's disease (PD) is the second most common neurodegenerative disease in humans. The cardinal symptoms of PD are tremor, rigor and bradykinesia (Parkinson, 2002)[reviewed in (Fahn, 2003)]. These symptoms are caused by a massive degeneration of dopaminergic (DA) neurons in the substantia nigra (SN). The $S N$ is part of the basal ganglia - a deep brain region that is involved in movement control - especially in the planning and initiation of movements (Brodal, 2004). Factors contributing to the particular vulnerability of DA neurons of the SN to cell death in PD will be discussed in section 2.2.1 of this introduction. At later stages of the disease, the pathology spreads to other brain regions and even at early stages, other parts of the brain can be affected. For example, patients often suffer from disturbances in olfaction before their movement becomes affected, which is also supported by histological examinations revealing histological alterations in the olfactory bulb at early stages of the disease (Braak et al., 2004). The major risk factor for developing PD is age. While the disease prevalence in Europe is about $1 \%$ for persons at the age of $65-70$, this value rises to $3-4 \%$ if a population between 80 and 90 years of age is considered (de Rijk et al., 1997). On the histological level, PD is characterized by the occurrence of proteinaceous inclusion bodies in the surviving dopaminergic neurons (Lewy, 1912) [reviewed in (Forno, 1996; Shults, 2006)]. These inclusions are called Lewy bodies (LBs) and are mainly composed of aggregated proteins. The picture of a LB is shown in Fig. 2.1.

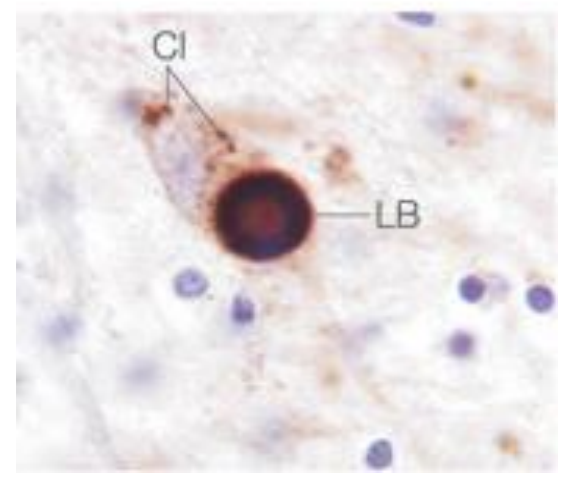

Fig. 2.1: Lewy bodies are the histological hallmarks of PD. a-synuclein staining reveals the presence of a large Lewy body (LB) and smaller cytoplasmic inclusions $(\mathrm{Cl})$ in $\mathrm{SN}$ neurons of PD patients. Reprinted by permission from Macmillan Publishers Ltd: [Nature Reviews Molecular Cell Biology] Ross and Poirier, copyright (2005). (Ross and Poirier, 2005).

The most abundant protein in LBs is a-synuclein (aS) (Baba et al., 1998; Spillantini et al., 1998; Spillantini et al., 1997; Takeda et al., 1998), a protein that has been linked to genetic cases of PD (Kruger et al., 1998; Polymeropoulos et al., 1997; Zarranz et al., 2004). As LBs are a very characteristic feature in post-mortem brains of PD patients, they were for a long time considered to be causative for neurodegeneration. However, it is important to keep in 
mind that the analysis of material from patients that have died with manifested PD can only deliver insight into late stages of the disease because symptomatic manifestation starts only after a considerable portion of SN nigra neurons have already died. Only those neurons that have survived over the course of the disease can be subjected to histological analysis. Therefore, it is even possible that inclusion of misfolded proteins into large bodies has a protective role when compared to smaller aggregates distributed throughout the cell. Currently, the treatment of $P D$ is symptomatic and aims in restoring DA levels in the striatum, the region to which DA neurons of the SN are projecting. This is achieved by treatment with L-DOPA, a precursor of DA that can pass the blood-brain-barrier and is metabolized to DA in the brain. Alternatively, COMT- (catechol-O-methyl transferase) and MAO-B- (monoamine oxidase B) inhibitors, which inhibit the degradation of DA, or DA agonists can be used for treatment of PD. However, all of these options are aiming to compensate the reduced DA levels but do not prevent further degeneration of neurons. A protective treatment that increases survival of the neurons would be desirable. In order to develop such treatments, it is necessary to first identify the molecular mechanisms that are involved in initial steps of the disease. Another task will then be to find biomarkers that allow an earlier diagnosis so that treatment could be started before the occurrence of massive neurodegeneration.

\subsubsection{Particular vulnerability of DA neurons in the SN}

The DA neurons in the SN of the midbrain seem to be particularly susceptible to neurodegeneration in PD. Although there are some other brain regions that also show signs of pathology like the locus coerulius (LC), the dorsal motor nucleus of the vagus or the basal nucleus of Meynert in the brain stem, DA neurons in the SN seem to be most severely affected. Three characteristic features of these neurons have been proposed as a reason for their particular sensitivity to PD pathogenesis.

First, DA neurons produce neuromelanin, a pigment that is responsible for the dark appearance of the SN. Neuromelanin is generated by the auto-oxidation of catecholamines, i.e. dopamine. Two nuclei in the human brainstem are exhibiting high levels of this pigment - the $\mathrm{SN}$ and the LC. Notably both are affected in PD. Although under physiological conditions neuromelanin might have a protective effect by chelating redox-active metal ions; it might become toxic under conditions of increased iron concentration as observed in PD patients (Dexter et al., 1991). It was proposed that in case of an overload with iron, neuromelanin promotes the generation of reactive oxygen species (ROS) (Double et al., 2002; Enochs et al., 1994; Youdim et al., 1994) More recently, neuromelanin was shown to inhibit the proteasomal system (Maruyama et al., 2006; Shamoto-Nagai et al., 2004) and, as dysfunction of 
the ubiquitin-protea $\neg$ some system (UPS) seems to be one of the main pathogenic mechanisms of PD, this inhibition might contribute to the increased vulnerability of the SN neurons. Furthermore, it was shown that neuromelanin induces oxidative stress in mitochondria and causes mitochondrial dysfunction (Shamoto-Nagai et al., 2004) and is thus also affecting the second major pathway of PD pathogenesis.

The second aspect for the increased susceptibility of SN DA neurons is their neurotransmitter DA or, more precisely, DA metabolites. DA metabolism leads to the formation of $\mathrm{H}_{2} \mathrm{O}_{2}$ and dopamine-quinone and these metabolic products are causing oxidative stress [reviewed in (Lotharius and Brundin, 2002)], thus rendering these neurons more vulnerable to PD pathogenesis, also going along with mitochondrial dysfunction and increased occurrence of ROS. Given the already high load of ROS in DA neurons due to the DA metabolism, toxic thresholds are reached faster in these cells. Furthermore, it was reported that DA can form covalent adducts with aS which in turn leads to the stabilization of proto-fibrillar variants of aS (Conway et al., 2001; Norris et al., 2005) that have been hypothesized to be the toxic species of aS (Volles and Lansbury, 2003).

The third characteristic feature of the DA neurons in the SN, which might explain their particular vulnerability, is that they exhibit a continuous pace-making activity that is driven by L-type $\mathrm{Ca}^{2+}$-channels (Chan et al., 2007). The $\mathrm{Ca}^{2+}$-ions that thereby enter the cell have to be transported out again, and this transport is ATP-dependent. Thus, an increased ATP production by the mitochondria in these neurons is required which in turn also leads to increased oxidative stress (Guzman et al., 2010). The basal ganglia neurons of the SN tend to oscillate and synchronize their activity (Brown, 2006). During these synchronous bursts of high activity SN neurons consume high amounts of oxygen for ATP production and therefore particularly rely on functional mitochondria. Thus, basal levels of ROS and the energetic requirements are increased in this cell type which leads to an increased sensitivity to oxidative stress and mitochondrial dysfunction emerging during PD pathogenesis.

\subsubsection{Genetics of $P D$}

Both environmental and genetic factors seem to play a role in the disease pathogenesis and aging is the major risk factor for developing PD. Only about $5-10 \%$ of PD cases suffer from familial forms of the disease (Corti et al., 2011; Toulouse and Sullivan, 2008), which in comparison to sporadic cases are often characterized by an earlier onset and sometimes also present with atypical signs such as dementia and dystonia. However, sporadic and genetic forms of PD share many symptomatic and histological features and it is thus assumed that the molecular mechanisms underlying disease pathogenesis are similar in the two forms. 
Therefore, investigation of the cellular function and toxicity of the genes that are linked to familial PD will also help to get a better understanding of the molecular pathways involved in disease pathogenesis of sporadic PD. To date, at least 11 genes have been associated to PD (Corti et al., 2011) and the most relevant of these will be discussed below. An overview of the PD-related genes is shown in Table 2.1. So far, two major pathophysiological pathways of PD have been described - disturbance in the UPS and increased protein aggregation on the one hand [reviewed in (Olanow and McNaught, 2006)] and disturbance of mitochondrial function and oxidative stress on the other hand [reviewed in (Bueler, 2009; Burbulla et al., 2010; Vives-Bauza et al., 2010a)]. An overview of the pathways involved in PD pathogenesis and the effects of different PD-associated genes on these pathways is depicted in Fig. 2.3 at the end of this section.

Table 2.1: Overview of PD-associated genes.

\begin{tabular}{|c|c|c|c|c|}
\hline $\begin{array}{l}\text { PARK } \\
\text { locus }\end{array}$ & Gene name & $\begin{array}{l}\text { C. elegans } \\
\text { ortholog }\end{array}$ & Protein class & Localization \\
\hline $\begin{array}{l}\text { PARK1/ } \\
\text { PARK4 }\end{array}$ & $\begin{array}{c}\text { SNCA } \\
\text { (a-synuclein) }\end{array}$ & no ortholog & Natively unfolded protein & $\begin{array}{c}\text { Presynaptic } \\
\text { Associated with membranes } \\
\text { Mitochondria }\end{array}$ \\
\hline PARK9 & ATP13A2 & catp-6 & P-type ATPase & Lysosomes \\
\hline PARK6 & PINK1 & pink-1 & Serine/Threonine Kinase & Mitochondria \\
\hline PARK2 & Parkin & pdr-1 & E3 Ubiquitin Ligase & $\begin{array}{c}\text { Cytosol } \\
\text { Depolarized mitochondria }\end{array}$ \\
\hline PARK7 & $D J-1$ & $\begin{array}{l}\operatorname{djr}-1.1 \\
\operatorname{djr}-1.2\end{array}$ & Redox-sensitive chaperone & $\begin{array}{c}\text { Cytosol } \\
\text { Mitochondria (during oxida- } \\
\text { tive stress) }\end{array}$ \\
\hline PARK8 & LRRK2 & Irk-1 & $\begin{array}{c}\text { ROCO protein } \\
\text { Kinase and GTPase }\end{array}$ & $\begin{array}{c}\text { Membranes, associated with } \\
\text { lipid rafts } \\
\text { Golgi (in C. elegans) }\end{array}$ \\
\hline PARK5 & $U C H-L 1$ & $u b h-1$ & Deubiquinating enzyme & Cytosol \\
\hline PARK11 & GIGYF2 & $\begin{array}{l}\text { no clear } \\
\text { ortholog }\end{array}$ & $\begin{array}{l}\text { Not known, might be involved } \\
\text { in tyrosine kinase signaling }\end{array}$ & Not known \\
\hline PARK13 & HtrA2/Omi & $\begin{array}{l}\text { no clear } \\
\text { ortholog }\end{array}$ & Mitochondrial protease & Mitochondria \\
\hline PARK14 & PLA2G6 & $\begin{array}{l}\text { no clear } \\
\text { ortholog }\end{array}$ & Phospholipase A2 & $\begin{array}{l}\text { Cytoplasm, can be translo- } \\
\text { cated to membranes }\end{array}$ \\
\hline PARK15 & FBXO7 & $\begin{array}{l}\text { no clear } \\
\text { ortholog }\end{array}$ & $\begin{array}{c}\text { F-box protein part of ubiquitin } \\
\text { ligase complex }\end{array}$ & $\begin{array}{l}\text { Cytoplasm } \\
\text { Nucleus }\end{array}$ \\
\hline
\end{tabular}




\section{a-synuclein}

The gene encoding $\alpha$-synuclein ( $\alpha S$ ), SNCA, was the first gene linked to autosomal dominantly inherited PD [reviewed in (Corti et al., 2003)]. Furthermore, aS was shown to be the predominant component of Lewy bodies (Baba et al., 1998; Spillantini et al., 1998; Spillantini et al., 1997; Takeda et al., 1998) and changes in the promoter region of the SNCA gene seem to be associated with sporadic forms of the disease (Mueller et al., 2005; Pals et al., 2004). It was shown, that increased expression levels of $\alpha S$, as observed in the case of multiplication of the locus, are sufficient to cause PD (Chartier-Harlin et al., 2004; Farrer et al., 2004; Ibanez et al., 2004; Singleton et al., 2003). Therefore, overexpression of aS has been used to generate animal models of the disease (Auluck et al., 2002; Lakso et al., 2003; Masliah et al., 2000).

$\alpha$-synuclein encodes for a small 140 aa protein which belongs to the group of natively unfolded proteins (Weinreb et al., 1996). aS can bind lipids (Kubo et al., 2005; Sharon et al., 2001) and adopts a more structured $\alpha$-helical conformation when associated to membranes (Eliezer et al., 2001). Furthermore, an interaction of aS with the presynaptic co-chaperone CSPa, which protects against high neuronal activity, was demonstrated [reviewed.in (Chandra and Südhof, 2008)] The protein is very prone to aggregation which involves formation $\beta$-sheet structures and which initially was thought to be the cause for toxicity. $\alpha S$ is a member of the synuclein protein family which also comprises $\beta$ - and $\gamma$-synuclein but only $\alpha S$ is associated to PD. Initially, aS was found as a component of amyloid plaques in Alzheimer's disease and was therefore called NCAP (non-amyloid beta-component of plaques) [reviewed in (Corti et al., 2011)]. The neuronal protein $\alpha \mathrm{S}$ is present at presynaptic terminals and several studies have implicated a role in regulating synaptic functions including vesicle release and DA metabolism (Murphy et al., 2000; Perez et al., 2002) [reviewed in (Stefanis, 2012)]. It was reported that expression of aS disturbs cellular membrane trafficking by blocking vesicle transport between endoplasmic reticulum (ER) and Golgi (Cooper et al., 2006) and Golgi fragmentation was reported to be caused by pre-fibrillar aS aggregates (Gosavi et al., 2002). Due to the sequence homology to 14-3-3 chaperone proteins, aS was proposed to function as a chaperone and this could be experimentally confirmed (Ostrerova et al., 1999; Souza et al., 2000). Investigation of the degradation of aS revealed that it can be degraded via the two cellular degradation pathways, the UPS and autophagy (Ebrahimi-Fakhari et al., 2011; Webb et al., 2003). Furthermore, it was reported that $\alpha$ S itself can inhibit both cellular degradation pathways, the UPS as well as autophagic degradation via lysosomes (Cuervo et al., 2004; Martinez-Vicente et al., 2008; Snyder et al., 2003; Winslow et al., 2010) thereby contributing to its own accumulation and toxicity. 


\section{ATP13A2}

Mutations in ATP13A2/PARK9 have been associated with Kufor-Rakeb syndrome (KRS), a parkinsonian syndrome that is characterized by more generalized brain atrophy than in typical PD and that causes additional symptoms aside the motor deficits that are seen in PD patients (Ramirez et al., 2006). Most of the mutations identified in KRS patients were found to lead to truncated variants of the protein and it was reported that these variants are retained in the ER instead of being transported to lysosomes, the physiological site of ATP13A2 action (Ramirez et al., 2006). Therefore, it can be assumed that the KRS mutations in ATP13A2 cause a strong loss of function of ATP13A2. Later on, heterozygous frame shift mutations and homo- as well as heterozygous missense mutations of ATP13A2 were also identified in PD patients (Di Fonzo et al., 2007; Djarmati et al., 2009; Fong et al., 2011; Lin et al., 2008).

ATP13A2 belongs to the superfamily of P-type ATPases which are involved in the transport of inorganic ions but also other substrates across membranes. P-type ATPases are characterized by the formation of a phosphorylated intermediate during the course of their reaction cycle (Axelsen and Palmgren, 1998; Kuhlbrandt, 2004). The domain structure of P-type ATPases comprises 10 transmembrane domains, an actuator domain, a phosphorylation domain and a nucleotide binding domain. In Fig. 2.2 the structure of P-type ATPases and a schematic drawing of the reaction cycle are depicted. The family of P-type ATPases is divided into five subfamilies according to their substrate specificities (Axelsen and Palmgren, 1998; Kuhlbrandt, 2004; Schultheis et al., 2004) and ATP13A2 belongs to the class of P5-type ATPases for which the substrate specificity remains to be determined (Axelsen and Palmgren, 1998; Kuhlbrandt, 2004; Moller et al., 2008). It was proposed that ATP13A2 is involved in transporting divalent cations like $\mathrm{Cd}^{2+}$ and $\mathrm{Mn}^{2+}$ based on the observation that in yeast, deletion of the ATP13A2 ortholog Ypk9 causes increased sensitivity to these cations (Gitler et al., 2009; Schmidt et al., 2009). At the time when this study was started, it was known that wild type ATP13A2 is localized to lysosomes and that truncated mutants accumulate in the ER, but the cellular function of the protein as well as the molecular mechanism causing toxicity in the mutants were uncharacterized. In 2009, Gitler et al. reported a functional link between ATP13A2 and aS with co-expression of ATP13A2 ameliorating aSinduced toxicity in several models (Gitler et al., 2009). 

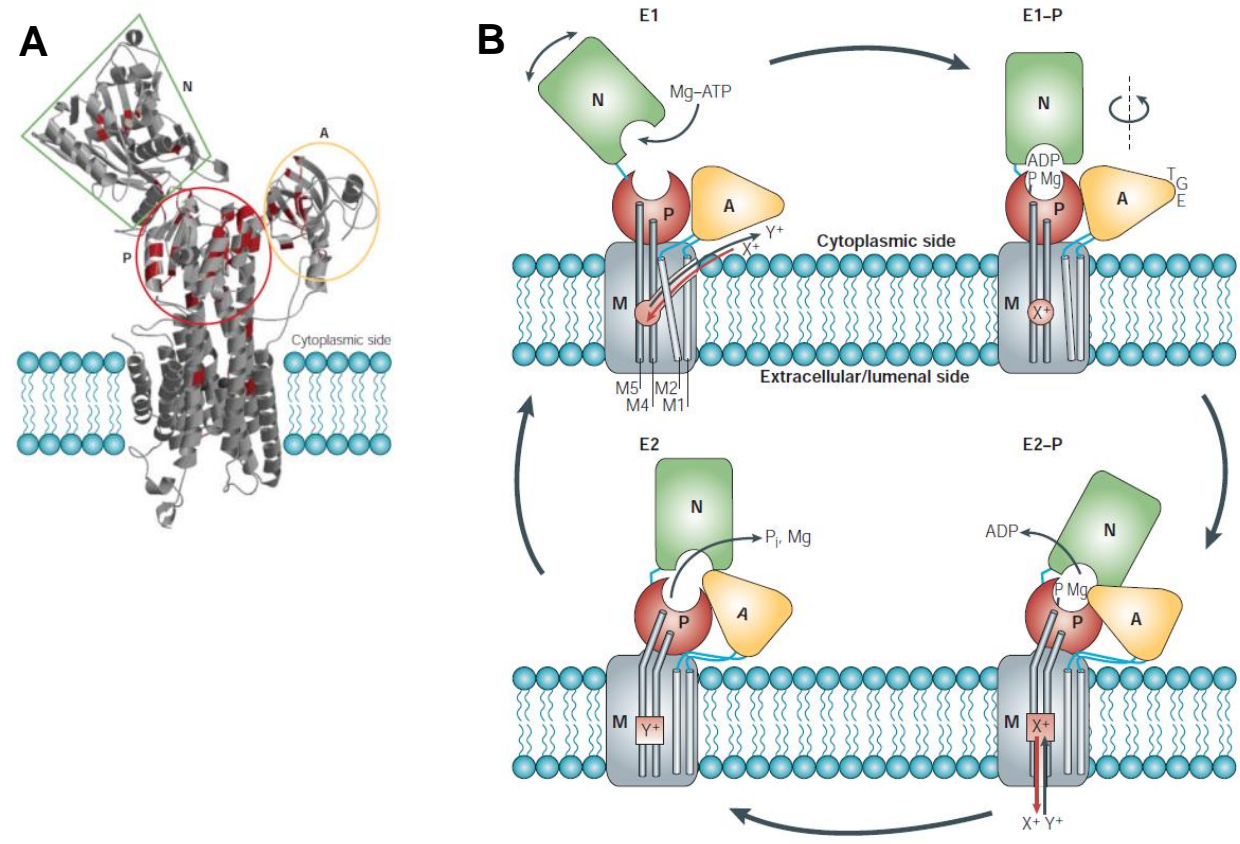

Fig. 2.2: Structure and reaction cycle of P-type ATPases.

(A) P-type ATPases are anchored in the membrane via ten transmembrane (TM) domains. The residues involved in ion binding are located inside the TM domains. Nucleotide binding domain ( $\mathrm{N}$; green), Phosphorylation domain (P; red) and actuator domain ( $\mathrm{A}$; yellow) are facing the cytosol. (B) The reaction cycle starts with the ATPase in $\mathrm{E} 1$ conformation and with ion $1\left(\mathrm{X}^{+}\right)$entering the ion binding site from the cytosolic side. The energy released by coordination of ion 1 induces a conformational change in the protein. $\mathrm{Mg}^{2+}$-ATP binds to the $\mathrm{N}$-domain and induces a change in conformation resulting in the $\mathrm{N}$-domain contacting the $\mathrm{P}$-domain (E1-P state). The Y-phosphate of ATP is subsequently transferred to a conserved Aspartate residue in the $P$ domain and this phosphorylation leads to transition of the enzyme to E2-P state in which the A-domain comes in close proximity to the P--domain which causes dissociation of ADP. A conformational change in the $\mathrm{P}$-domain results in release of ion 1 to the extracellular/luminal side. Subsequently, ion $2\left(\mathrm{Y}^{+}\right)$enters the ion binding site and the phosphorylated Aspartate is hydrolyzed. $\mathrm{P}_{\mathrm{i}}$ and $\mathrm{Mg}^{2+}$ are released and the enzyme is adopting E2 conformation. Opening of the ion binding site and release of ion 2 brings the ATPase back into the E1 state and the cycle can be started again. Reprinted with permission from Macmillan Publishers Ltd: [Nature Reviews Molecular Cell Biology] Kuhlbrandt, copyright (2004). (Kuhlbrandt, 2004).

\section{PINK1}

PINK1 (PTEN-induced putative kinase 1) is a Serine/Threonine kinase that has been linked to early-onset autosomal recessive PD. A mitochondrial targeting sequence is located at the $\mathrm{N}$-terminus of the protein and mitochondrial localization was shown in several studies (Clark et al., 2006; Silvestri et al., 2005) Mutations in PINK1 have been found in familial cases of PD (Valente et al., 2004a) but also in patients suffering from sporadic PD and it was proposed that heterozygous mutations in PINK1 might be a risk factor for PD (Abou-Sleiman et al., 2006; Djarmati et al., 2006; Valente et al., 2004b). Most of the mutations that have been identified reside in the kinase domain of PINK1 and for some of them a reduced kinase activity was reported (Beilina et al., 2005; Sim et al., 2006). This observation, together with the autosomal recessive pattern of inheritance, suggests that PD mutations are causing a loss of 
function of PINK1 (Sim et al., 2006). Knockout (KO) of the Drosophila ortholog of PINK1 was described to induce mitochondrial abnormalities, muscle degeneration and reduced fertility (Clark et al., 2006; Park et al., 2006; Yang et al., 2006). Interestingly, KO of Parkin in Drosophila caused similar phenotypes (Greene et al., 2003) and it was shown that the phenotypes induced by PINK1 KO could be rescued by overexpression of parkin but not vice versa (Clark et al., 2006; Park et al., 2006; Yang et al., 2006) suggesting that the two proteins function in the same pathway with parkin being downstream of PINK1. Later on, it was shown that the PINK1/Parkin pathway is involved in targeting damaged mitochondria for degradation via mitophagy, which will be discussed in section 2.2.3 of this introduction.

\section{Parkin}

Parkin is an E3 ubiquitin ligase and has been linked to autosomal recessive, early-onset PD (Kitada et al., 1998; Matsumine et al., 1997). Several mutations in Parkin have been identified in PD patients including deletions, multiplications and missense mutations (Kitada et al., 1998)[reviewed in (Mata et al., 2004)]. It was shown that Parkin plays a role in protein degradation via the proteasome and PD-associated mutations in Parkin cause loss of ubiquitin ligase activity and thus accumulation of its substrates (Shimura et al., 2000; Zhang et al., 2000). Similar to PINK1 KO, loss of Parkin in flies leads to mitochondrial defects as well as muscle degeneration, sperm defects and a reduced life span (Greene et al., 2003). As already described in the PINK1 section, PINK1 and Parkin are involved in the same pathway (mitophagy, see section 2.2.3) and PINK1 is upstream of Parkin (Clark et al., 2006; Park et al., 2006; Yang et al., 2006). Besides the functional interaction with PINK1, Parkin was also attributed a protective effect against aS-induced toxicity (Haywood and Staveley, 2006; Petrucelli et al., 2002; Yang et al., 2003).

\section{DJ-1}

Mutations in $D J-1$ have been identified in patients suffering from autosomal recessive, earlyonset PD (Bonifati et al., 2003b) and it was shown that PD-associated mutations in DJ-1 interfere with dimerization, thereby impairing its function (Moore et al., 2005; Olzmann et al., 2004). DJ-1 acts as antioxidant and redox-sensitive chaperone that is modified in conditions of oxidative stress (Bonifati et al., 2003a; Canet-Aviles et al., 2004; Mitsumoto and Nakagawa, 2001; Shendelman et al., 2004). A protective function of DJ-1 against oxidative stress was observed in Drosophila (Meulener et al., 2006) whereas loss of function of DJ-1 renders cells more vulnerable to oxidative stress but also ER stress, inhibition of the proteasome or induction of apoptosis (Andres-Mateos et al., 2007; Yokota et al., 2003). 


\section{LRRK2}

LRRK2 (Leucine-Rich Repeat Kinase 2) is associated with autosomal dominantly inherited PD of late onset (Di Fonzo et al., 2005; Funayama et al., 2002; Zimprich et al., 2004). LRRK2 belongs to the family of ROCO proteins that are characterized by the presence of a ROC (Ras of complex protein) and a COR domain (C-terminal of ROC) (Marin et al., 2008). The ROC domain is a GTPase that, together with the COR domain, plays a role in intra-molecular regulation of the LRRK2 kinase activity and LRRK2 was shown to form dimers (Deng et al., 2008; Greggio et al., 2008; Guo et al., 2007). Besides the ROC/COR and kinase domains, LRRK2 also contains several protein-protein interaction domains like LRR (Leucine rich repeat), WD40 and ankyrin repeat domains (Kumar and Cookson, 2011; Mata et al., 2006). Mutations in the LRRK2 gene not only account for rare familial form of PD but have also been identified in sporadic cases (Gilks et al., 2005; Lesage et al., 2007). Most of the LRRK2 mutations are located in the GTPase and kinase domain and for several of them an increased kinase activity was demonstrated (Gloeckner et al., 2006; West et al., 2005; West et al., 2007). Moreover, inhibition of kinase activity was shown to be protective against LRRK2mediated toxicity (Smith et al., 2006; West et al., 2007) indicating that a gain-of-function is responsible for disease pathogenesis. LRRK2 localizes to membranes and associates with lipid rafts (Biskup et al., 2006; Hatano et al., 2007). Mitochondrial localization of the protein was reported in human cell lines and mouse brains (Biskup et al., 2006; West et al., 2005) and a recent study has revealed impaired mitochondrial function and dynamics in fibroblasts of PD patients carrying mutations in LRRK2. Mitochondria in these cells were more elongated and interconnected than in cells from healthy controls and reduced membrane potential and ATP levels were reported (Mortiboys et al., 2010). Moreover, LRRK2 has been found to regulate neurite outgrowth (MacLeod et al., 2006) and deletion of the C. elegans ortholog Irk-1 resulted in mistargeting of SV proteins to dendrites (Sakaguchi-Nakashima et al., 2007).

\section{UCH-L1}

A missense mutation in UCH-L1 (Ubiquitin carboxyl-terminal hydrolase L1) was identified in only one PD family (Leroy et al., 1998) and a polymorphism at another residue was found to decrease the risk for developing sporadic PD at least in some studies (Carmine Belin et al., 2007; Elbaz et al., 2003; Facheris et al., 2005). UCH-L1 is part of the UPS and is involved in cleavage of small adducts of ubiquitin thereby generating free monomeric ubiquitin (Larsen et al., 1998). The disease-causing mutation was found to exhibit a decreased hydrolytic activity while the protective polymorphism caused an increase in the catalytic activity, suggesting that loss of enzyme activity is involved in disease pathogenesis (Leroy et al., 1998; Nishikawa et al., 2003). Interestingly, UCH-L1 was also reported to be a component of Lewy bodies further substantiating its link to PD. 


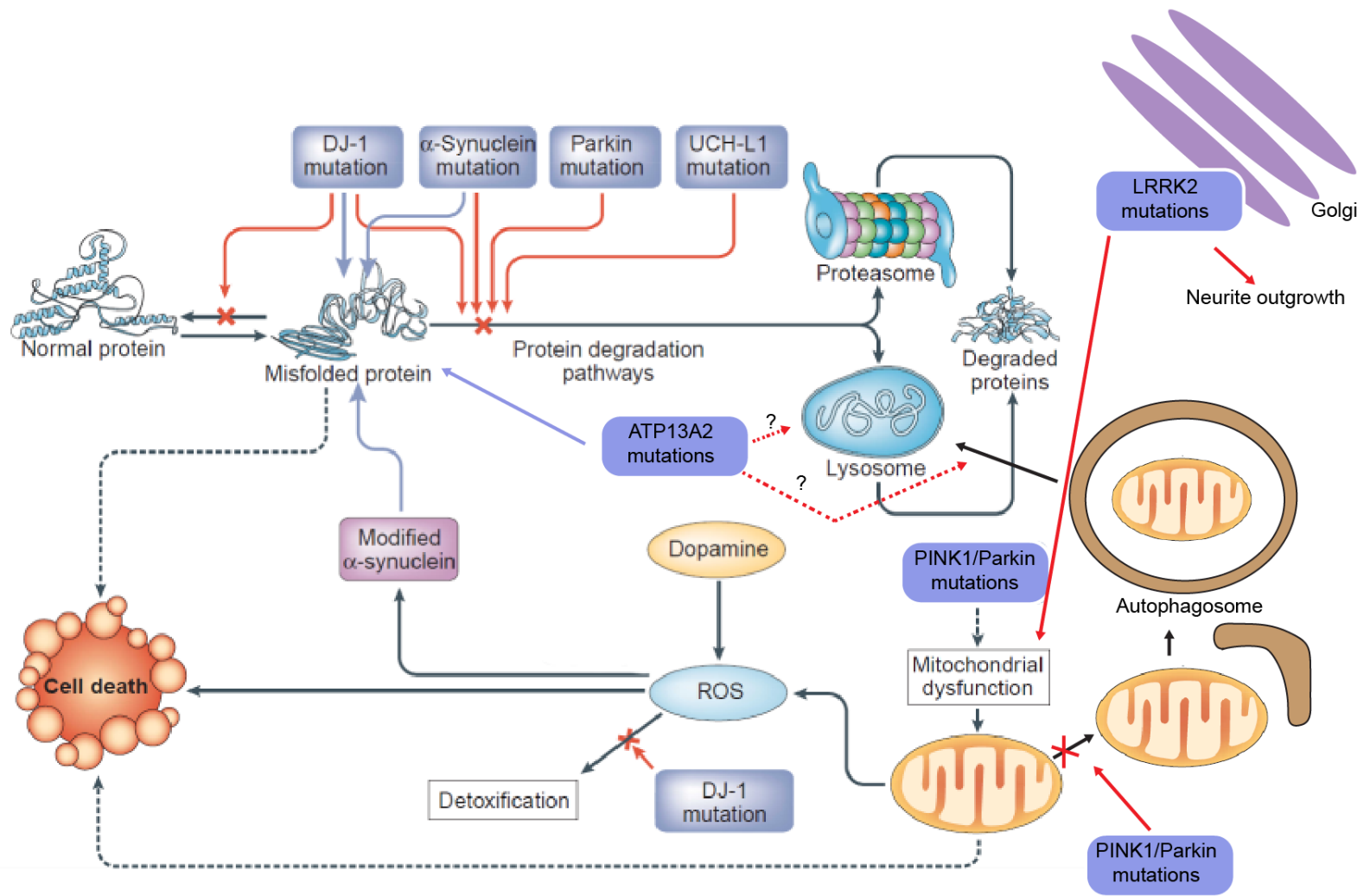

Fig. 2.3: Pathways involved in PD pathogenesis and PD-associated genes affecting these pathways.

The two major pathways causing cell death in PD are mitochondrial dysfunction (lower half of the schematic drawing) as well as accumulation of misfolded proteins and inhibition of the cellular degradation pathways (upper half of the diagram). PD-related genes that have been linked to these mechanisms are depicted in blue boxes and arrows indicate how they interfere with the above mentioned pathways. Red arrows represent inhibitory effects or disturbance the organelle's function; blue arrows are representing stimulating effects. DJ-1 mutations interfere with re-folding of misfolded proteins and also inhibit degradation of misfolded proteins thereby causing accumulation of misfolded proteins. Furthermore, DJ-1 is involved in the detoxification of ROS and mutations interfere with this process causing increased protein damage and misfolding. aS mutations cause aggregation of the protein and also inhibit the protein degradation machinery. Mutations in Parkin and UCH-L1 also have an inhibitory effect on protein degradation and mutations in ATP13A2 were shown to stimulate aS aggregation but are also thought to interfere with lysosomal function and autophagic clearance. LRRK2 was shown to be localized to the Golgi and mutations are reducing neurite outgrowth and also cause mitochondrial dysfunction. Mitochondrial dysfunction and comprised clearance of damaged mitochondria are caused by mutations in Parkin and PINK1. Mitochondrial dysfunction also leads to increased ROS production which results in oxidative damage to proteins and higher loads of misfolded proteins. Adapted by permission from Macmillan Publishers Ltd: [Nature Medicine] Vila and Przedborski, copyright (2004). (Vila and Przedborski, 2004). 


\subsection{Mitochondria}

Mitochondria are cellular organelles that differ from other cellular compartments in that they are enclosed by a double membrane. Furthermore, mitochondria have their own genome and possess a protein synthesis machinery, which is similar to that of prokaryotes. Only a small fraction of mitochondrial proteins is synthesized inside the mitochondrion, while the rest is encoded by nuclear genes, translated in the cytosol and then imported into the mitochondria. These special properties of the mitochondria are due to their origin - according to the endosymbiont theory they originate from prokaryotes that were engulfed by an ancestral eukaryotic cell [reviewed in (Gray and Doolittle, 1982)]. The structure of mitochondria can be divided in four components: outer mitochondrial membrane (OMM), intermembrane space (IMS), inner mitochondrial membrane (IMM) and mitochondrial matrix. The surface of the IMM is enlarged by invaginations, the so-called cristae, and contains the complexes of the respiratory chain which is illustrated in Fig 2.4. Mitochondrial biogenesis does not occurs "de novo" but is achieved by incorporation of new components into already existing mitochondria while damaged material is removed via the cellular degradation systems.

\subsubsection{Mitochondrial function}

Mitochondria are often described as "cellular power plants" because of their function in providing energy in form of ATP to the cell. However, the functions of mitochondria are by far more diverse - they are involved in a number of anabolic and catabolic processes as well as in cellular signaling pathways and in controlling apoptotic cell death. Catabolic processes taking place in the mitochondrial compartment are the citric acid cycle, $\beta$-oxidation of fatty acids and oxidative phosphorylation at the respiratory chain. A schematic drawing of the respiratory chain is depicted in Fig. 2.4. Among the mitochondrial anabolic processes are the synthesis of iron-sulfur clusters, heme and lipids. Another important property of mitochondria is their capacity of calcium buffering which helps to regulate the intracellular $\mathrm{Ca}^{2+}$ homeostasis. As mitochondria are essential for a plethora of cellular processes, regulation and maintenance of mitochondrial function is very important for the cell. 


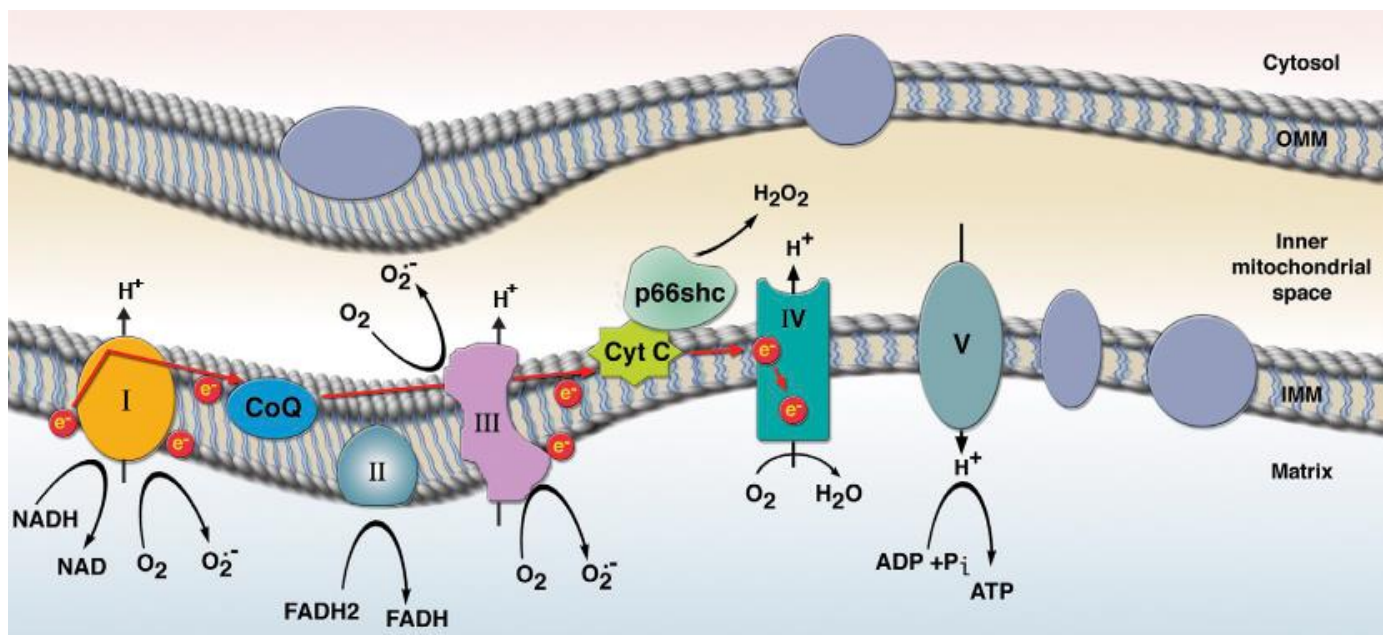

\section{Fig. 2.4: Mitochondrial electron transfer chain.}

The mitochondrial electron transfer chain, also known as respiratory chain, resides in the inner mitochondrial membrane and comprises several complexes involved in ATP production via oxidative phosphorylation. The major principle of oxidative phosphorylation is transfer of electrons from a substrate to molecular oxygen resulting in the generation of water. Depending on the substrate, electrons enter the respiratory chain either at complex I (NADH-dehydrogenase) or at complex II (Succinate dehydrogenase) and are then passed to complex III (Ubiquinol cytochrome c oxidoreductase). In the case of electrons that have entered the chain via complex I, they are first transferred to Coenzyme $Q$ and from there are transferred to complex III. Next, electrons are passed on to complex IV (Cytochrome c oxidase) via an intermediary substrate cytochrome c. At complex IV, the electrons are finally transferred to molecular oxygen which results in the generation of water. The energy that is freed during electron transfer through the respiratory chain is used to build up a proton gradient across the IMM. Protons are pumped in the IMS at complexes I, III and IV and the proton gradient is subsequently used by the ATP synthase to generate ATP from ADP $+P_{i}$. Electrons can leak out of the respiratory chain before the final transfer to molecular oxygen at complex IV. This primarily happens at complex I and complex III and results in superoxide production, a ROS species. CFinkel, 2011. Originally published in The Journal of Cell Biology. doi: 10.1083/jcb.201102095. (Finkel, 2011).

\subsubsection{Mitochondrial dynamics}

Although typical textbook pictures of mitochondria depict this organelle in a rod-like shape, mitochondrial morphology is by far more diverse, ranging from small vesicular structures to networks of elongated and highly interconnected tubules, and can be adapted to the cellular energy demands [reviewed in (Sauvanet et al., 2010)]. Mitochondrial morphology is also highly dependent on the cell type. Mitochondria are not static but highly dynamic and constantly undergoing fusion and division and the balance between fusion and fission events determines mitochondrial shape in a cell [reviewed in (Cerveny et al., 2007)]. A number of studies in the past decade have shed light on the molecular machineries that are controlling mitochondrial dynamics and a growing number of reviews are concerned with this topic. The key players involved in regulation of mitochondrial fission and fusion will be introduced below 
and the reader is referred to review articles on mitochondrial dynamics (Chan, 2006; Hoppins et al., 2007; Westermann, 2010a, b) for more detailed information.

\section{Mitochondrial fusion}

Mitochondrial fusion can be subdivided into two events, namely fusion of the OMM and subsequent fusion of the IMM. The two processes seem to be coupled under normal conditions but can also occur independently from each other [reviewed in (Griffin et al., 2006)].
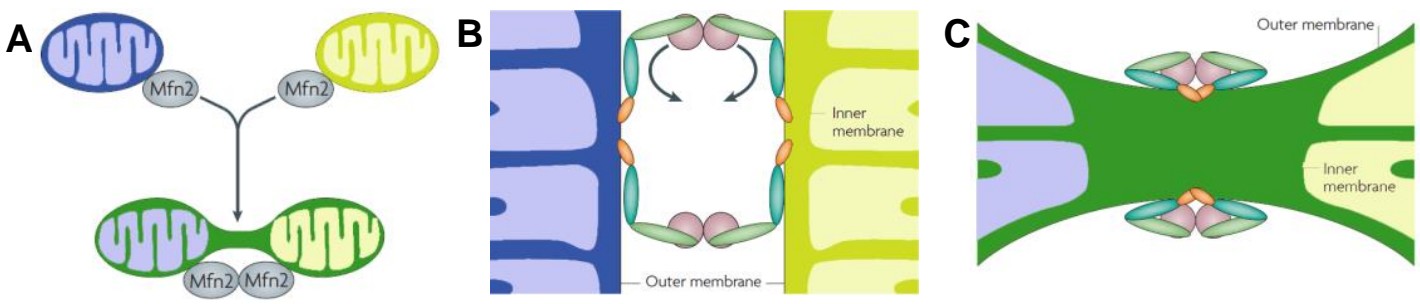

Fig. 2.5: Fusion of the mitochondrial outer membranes is mediated by mitofusins in mammals.

(A) Mitochondrial outer membrane fusion is mediated by mitofusins located the outer membranes of opposing mitochondria. Fusion results in mixing of the outer membrane components while the matrices remain separated.

(B) Mitofusins are tethering the membranes thus bringing them in close proximity to each other. (C) GTPase activity of the mitofusins results in a conformational change that leads to fusion of the mitochondrial membranes. Adapted by permission from Macmillan Publishers Ltd: [Nature Reviews Neuroscience] Knott et al., copyright (2008). (Knott et al., 2008).

As depicted in Fig. 2.5, outer membrane fusion in mammals is mediated by the mitofusins Mfn1 and Mfn2, which belong to a group of large dynamin-related GTPases and which are localized to the OMM (Chen et al., 2003a) [reviewed in (Hoppins et al., 2007)]. Mitofusins on opposing mitochondrial membranes are forming a complex in trans, which results in tethering of the membranes and GTPase activity induces fusion of the membranes [reviewed in (Griffin et al., 2006; Meeusen and Nunnari, 2005)]. In C. elegans, OMM fusion is mediated by just one mitofusin ortholog, which is called fzo-1 (Hales and Fuller, 1997).

In mammals, fusion of the IMM, as depicted in Fig. 2.6, is mediated by OPA1 (optic atrophy 1) which, like the mitofusins, belongs to the family of dynamin-related GTPases [reviewed in (Chan, 2006)]. OPA1 activity requires the presence of Mfn1 (Cipolat et al., 2004) and again, complexes between OPA1 molecules in trans result in tethering of the membranes and subsequent membrane fusion [reviewed in (Hoppins et al., 2007)]. OPA1 can be cleaved by several mitochondrial proteases resulting in long and short isoforms of the protein and this posttranslational processing seems to be an important mechanism for the regulation of OPA1 activity [reviewed in (Cerveny et al., 2007; Hoppins et al., 2007)]. The C. elegans ortholog of OPA1 is eat-3 (Kanazawa et al., 2008). 


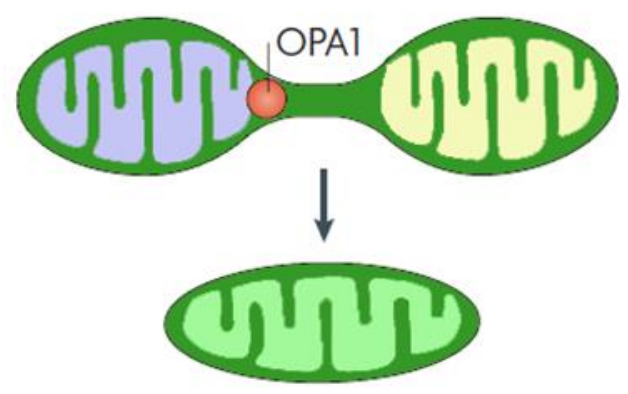

Fig. 2.6: OPA1 mediates inner membrane fusion in mammals.

After successful fusion of the OMM, the GTPase OPA1 mediates fusion of the IMM in a process that is dependent on GTPase activity of OPA1. Adapted by permission from Macmillan Publishers Ltd: [Nature Reviews Neuroscience] Knott et al., copyright (2008). (Knott et al., 2008).

\section{Mitochondrial fission}

The cytosolic dynamin-related GTPase Drp1 (Dynamin-related protein 1) is recruited to mitochondria to mediate mitochondrial fission. At the mitochondria, Drp1 oligomerizes and forms ring-like structures around the mitochondrion. GTPase activity subsequently leads to constriction of the membranes in a process that resembles dynamin-mediated constriction of vesicles during clathrin-mediated endocytosis [reviewed in (Lackner and Nunnari, 2009)]. A schematic drawing of Drp1-mediated mitochondrial fission is shown in Fig. 2.7.

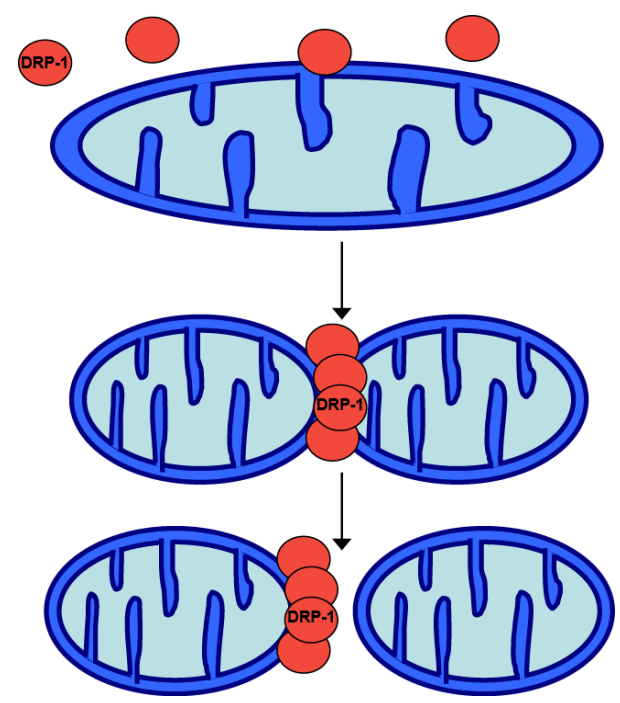

Fig. 2.7: Mitochondrial fission is mediated by Drp1. Mitochondrial fission is initiated by translocation of Drp1 to the mitochondrial membrane where it assembles into ring-like structures and subsequently mediates constriction of the membranes by GTPase activity.

In yeast, mitochondrial targeting of the Drp1 ortholog Dnm1 is mediated by the protein Fis1 (Mitochondrial fission 1) which is anchored in the OMM and recruits Dnm1 via the adaptor proteins Caf4 (CCR4 associated factor 4) and Mdv1 (Mitochondrial division protein 1) [reviewed in (Lackner and Nunnari, 2009)]. An ortholog of Fis1 has also been identified in mammals however it remains controversial whether it also functions in recruiting Drp1 to the sites of fission as no adaptor proteins have been identified so far and Fis1 seems to localize diffusely to mitochondrial membranes in contrast to punctate pattern of recruited Drp1 [reviewed in (Otera and Mihara, 2011)]. 
Fission activity of Drp1 has been shown to be regulated by several posttranslational modifications including phosphorylation, S-nitrosylation, ubiquitination and sumoylation [reviewed in (Otera and Mihara, 2011; Santel and Frank, 2008)]. As illustrated in Fig. 2.8, the phosphorylation status of Ser637 of Drp1 has been demonstrated to play an important role in regulating mitochondrial fission activity. This residue is located in the GED domain (GTPase effector domain) and phosphorylation impairs intra-molecular interaction of the GED domain with the GTPase domain which causes decreased GTPase activity [reviewed in (Otera and Mihara, 2011)]. While Drp1 that is phosphorylated at this residue is retained in the cytosol and cannot mediate fission, dephosphorylation results in translocation of Drp1 to the mitochondria thereby stimulating mitochondrial fission activity [reviewed in (Otera and Mihara, 2011)]. It was demonstrated that phosphorylation of Drp1 at Ser637 is mediated by cAMPactivated protein kinase $\mathrm{A}$ (PKA) and that a phosphomimetic variant of Drp1 (Ser637Asp) inhibits mitochondrial fission in cell culture (Chang and Blackstone, 2007; Cribbs and Strack, 2007). Dephosphorylation of Drp1 Ser637 is mediated by calcineurin (Cereghetti et al., 2008; Cribbs and Strack, 2007) and expression of Drp1 Ser637Ala, which cannot be phosphorylated, results in increased mitochondrial fission (Cribbs and Strack, 2007).

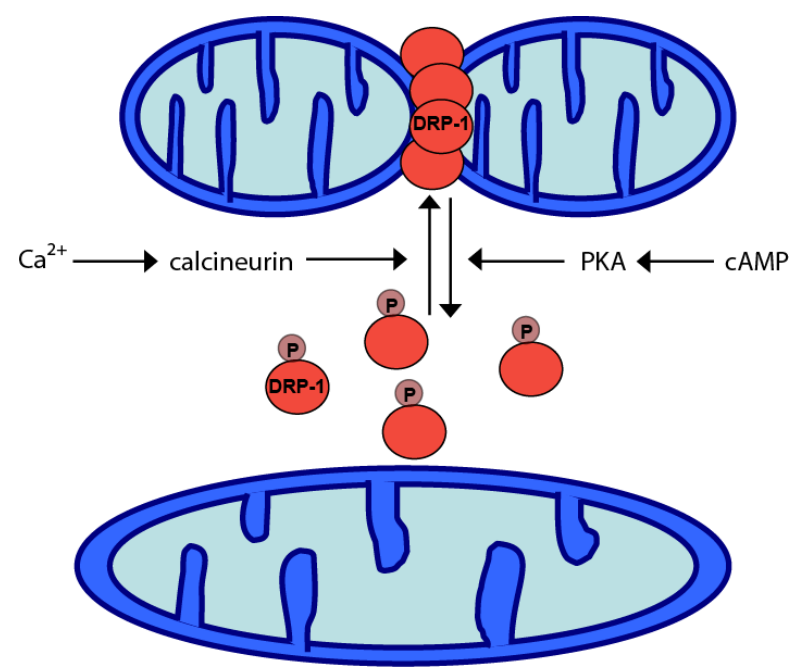

Fig. 2.8: Phosphorylation status of Drp1 regulates mitochondrial fission activity.

Phosphorylation of Drp1 prevents mitochondrial translocation and thus inhibits mitochondrial division. Phosphorylation of Ser637 is mediated by PKA in response to a rise in cAMP levels while $\mathrm{Ca}^{2+}$-mediated activation of calcineurin promotes dephosphorylation of Drp1 thereby stimulating mitochondrial translocation and fission activity.

\section{Mitochondrial transport}

Besides fusion and division, mitochondrial transport is also contributing to the dynamic nature of this organelle by controlling distribution of mitochondria. Mitochondrial transport is especially important in neurons where presence of mitochondria in the neurites is required to ensure ATP supply and $\mathrm{Ca}^{2+}$-homeostasis for proper synaptic function. Mitochondrial transport predominantly occurs along microtubules but in mammalian neurons it also depends on actin [reviewed in (Dimmer and Scorrano, 2006)]. While intact mitochondria are primarily transported in the anterograde direction by kinesin, dynein motors mediate retro- 
grade transport of damaged mitochondria back to the cell body where they are degraded or complemented by fusion with intact mitochondria [reviewed in (Otera and Mihara, 2011)]. The proteins Miro and Milton are involved in mitochondrial transport and a $\mathrm{Ca}^{2+}$-binding motif of Miro plays an important role in adapting mitochondrial motility to local $\mathrm{Ca}^{2+}$-concentrations [reviewed in (Dimmer and Scorrano, 2006; Otera and Mihara, 2011)]. Balanced fission and fusion of mitochondria is crucial for mitochondrial transport and lack of either fission or fusion strongly impairs mitochondrial transport [reviewed in (Chen and Chan, 2006)].

\section{Function of mitochondrial fission and fusion}

Mitochondrial fusion enables the exchange of components between mitochondria which allows for complementation of defective components in one of the mitochondria and thereby helps to keep mitochondria in a functional state [reviewed in (Otera and Mihara, 2011)]. Accordingly, inhibition of fusion results in impaired respiratory chain activity [reviewed in (Otera and Mihara, 2011)]. Besides enabling complementation, mitochondrial fusion has also a protective function against apoptosis which involves increased mitochondrial fragmentation [reviewed in (Chen and Chan, 2006)].

In contrast, mitochondrial fission plays a role in mediating proper distribution of the mitochondria but also serves to ensure degradation of damaged mitochondrial components by separating damaged from intact material so that subsequent mitophagy of the defective part can take place [reviewed in (Otera and Mihara, 2011)]. Mitochondrial division is also required for equal sequestration of mitochondria into the daughter cells during cell division [reviewed in (Otera and Mihara, 2011)] and deletion of the C. elegans ortholog drp-1 causes embryonic lethality which can be at least in part attributed to defects in mitochondrial segregation [reviewed in (Lackner and Nunnari, 2009)]. Finally Drp1-mediated fission is an early step of apoptosis and blocking mitochondrial fission by expression of DN Drp1 is protective against apoptotic cell death [reviewed in (Youle and Karbowski, 2005)].

Loss of the mitochondrial fusion genes has been associated to neurodegeneration. In humans, mutations in mitofusins have been identified as cause for Charcot-Marie-Tooth disease type 2A, a neurodegenerative disease of the peripheral nerves (Zuchner et al., 2004) and loss of OPA1 is associated with dominant optic atrophy a disease which is characterized by degeneration of the retinal ganglion cells (Alexander et al., 2000; Delettre et al., 2000). It was also reported that a mutation in the fission protein Drp1 causes microcephaly, abnormal brain development and optic atrophy resulting in lethality (Waterham et al., 2007). This demonstrates that well-balanced mitochondrial dynamics are essential for ensuring mitochondrial function and several pathologic conditions like PD, Alzheimer's disease and Hun- 
tington's disease have been associated with disturbances of the fission/fusion systems [reviewed in (Cho et al., 2010)].

\subsubsection{Mitochondrial quality control - stress response pathways and mitophagy}

\section{Mitochondrial stress response}

The mitochondrial stress response is a compartment-specific pathway to react to an overload of unfolded proteins. It belongs to a number of other cellular stress responses like the cytosolic heat shock response and the unfolded protein response in the ER (UPR ${ }^{\mathrm{ER}}$ ) [reviewed in (Broadley and Hartl, 2008)] and they all are induced by a disequilibrium in protein folding which results in high loads of un- and misfolded proteins. A number of studies have investigated the mitochondrial unfolded protein response $\left(U_{P R}{ }^{m t}\right)$ in $C$. elegans and have shed light on the processes involved in this pathway and an illustration of the pathway is shown in Fig. 2.9. Yoneda et al. demonstrated that disturbance of protein handling inside mitochondria by knockdown of genes encoding mitochondrial chaperones or proteases leads to increased expression of the mitochondrial chaperones HSP-6 (C. elegans ortholog of human mitochondrial Hsp70) and HSP-60 (C. elegans ortholog of human Hsp60) (Yoneda et al., 2004). Subsequently, ubl-5, a ubiquitin-like protein was identified in an RNAi screen for genes involved in the induction of the UPR ${ }^{\mathrm{mt}}$. Knockdown of $u b l-5$ prevented induction of the mitochondrial stress response and it was furthermore demonstrated that in wild type worms, UBL-5 is translocated to the nucleus upon induction of the mitochondrial stress response (Benedetti et al., 2006). Haynes et al. identified another cytosolic protein, the transcription factor DVE-1 which plays an important role in the UPR ${ }^{\mathrm{mt}}$. Upon induction of the mitochondrial stress response, DVE-1 forms a complex with UBL-5 and is translocated to the nucleus where DVE-1 stimulates expression of the mitochondrial chaperones HSP-6 and HSP-60 (Haynes et al., 2007). Haynes et al. furthermore demonstrated that silencing of the mitochondrial protease CLPP-1 interferes with induction of the mitochondrial stress response upstream of DVE-1 nuclear translocation. They proposed a model in which peptides generated by cleavage of mitochondrial proteins by CLPP-1 are mediating the signaling to the cytosol that is needed for activation of the stress response (Haynes et al., 2007). In 2010, another study by Haynes et al. revealed that a mitochondrially localized peptide transporter, HAF-1, as well as the transcription factor bZIP also play a role in activation of the UPR ${ }^{\mathrm{mt}}$ suggesting that peptides generated by CLPP-1-mediated cleavage are transported to the cytosol where they induce signaling to activate the mitochondrial stress response (Haynes et al., 2010). The target genes of the bZIP transcription factor ZC376.7 are not known by now. 


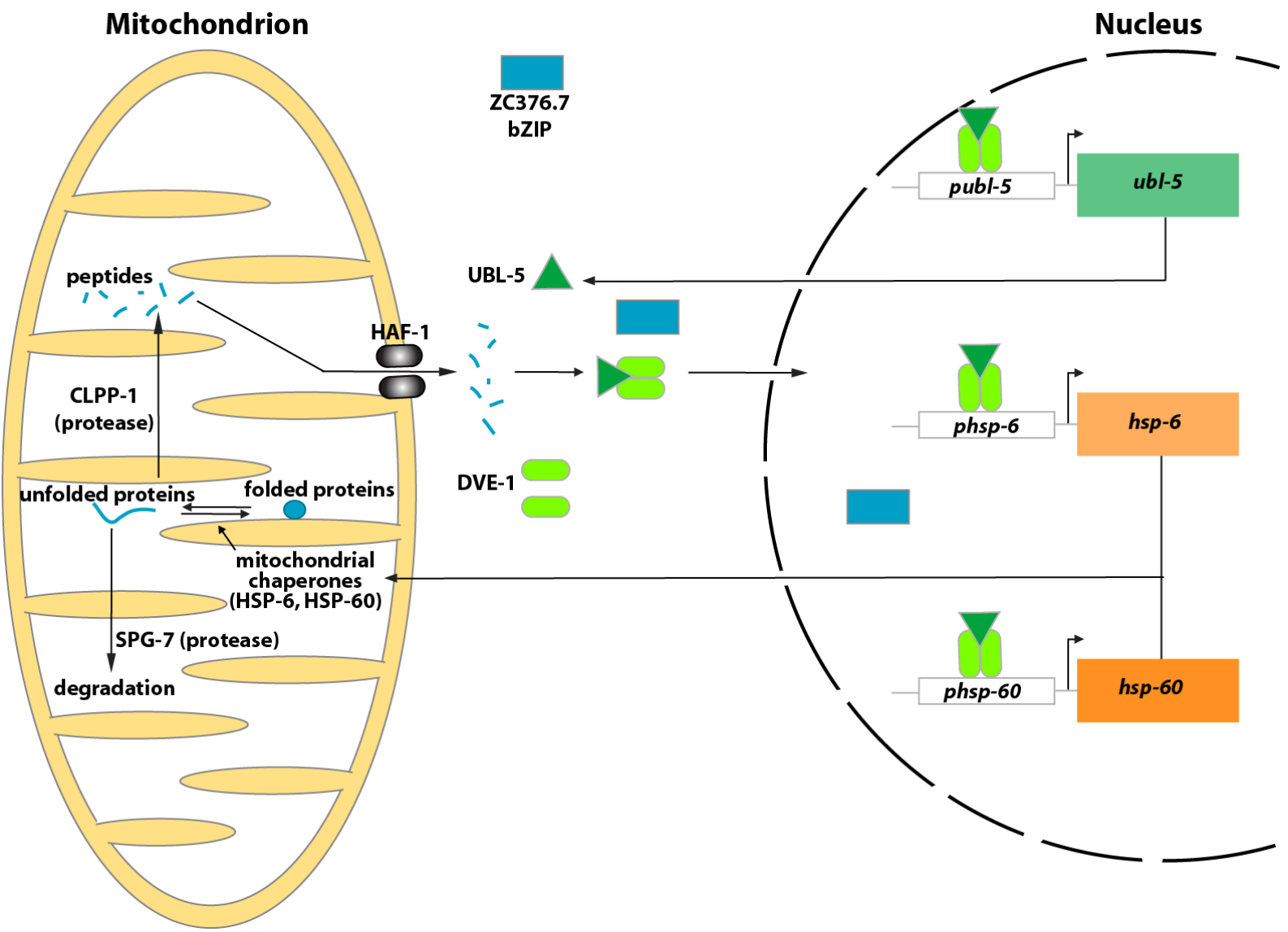

Fig. 2.9: Mitochondrial unfolded protein response $\left(U P R^{\mathrm{mt}}\right)$ in $C$. elegans.

The $U_{P R}{ }^{\mathrm{mt}}$ is induced by an overload of misfolded proteins in the mitochondria. Misfolded proteins are either removed by degradation by SPG-7, a mitochondrial protease, or are cleaved into small peptides by another mitochondrial protease, CLPP-1. The small peptides subsequently are released into the cytosol via the ABC transporter HAF-1 and trigger formation of a complex of the transcription factor DEV-1 and UBL-5, a ubiquitin-like protein. This complex as well as another transcription factor, ZC376.7 translocate to the nucleus where DVE-1 stimulates expression of the mitochondrial chaperones HSP-6 and HSP-60. The chaperones are imported into the mitochondria and help to re-establish proper protein folding. The targets of the bZIP transcription factor ZC376.7 are not yet known.

\section{Mitophagy}

Another quality control system for mitochondria is the selective degradation of mitochondria via mitophagy. Mitophagy is a special form of autophagy and relies on the same core machinery as macroautophagy [reviewed in (Kanki and Klionsky, 2010)]. Mitophagy, similar to autophagy can be induced under conditions of starvation but was also found to serve in clearance of damaged mitochondria under normal conditions [reviewed in (Tanaka, 2010)]. Intriguingly, two PD-related proteins, PINK1 and Parkin play an essential role in the specific targeting of damaged mitochondria for elimination via mitophagy [reviewed in (Springer and Kahle, 2011)]. A prerequisite for clearance of damaged mitochondrial material is its separation from intact components. It was shown that this can be achieved by mitochondrial fission 
generating uneven daughter-organelles, one containing intact material while the other is comprised of damaged material and is characterized by a reduced membrane potential (Twig et al., 2008a). Fusion of these mitochondria with intact organelles is prevented due to lack of OPA1 activity at the damaged mitochondrion (Twig et al., 2008a).

A

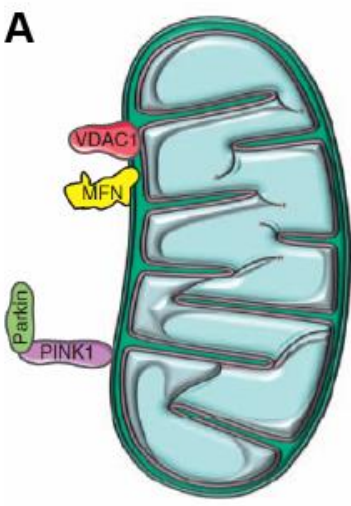

B

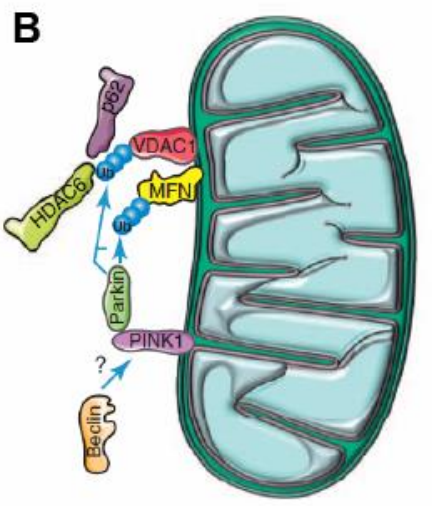

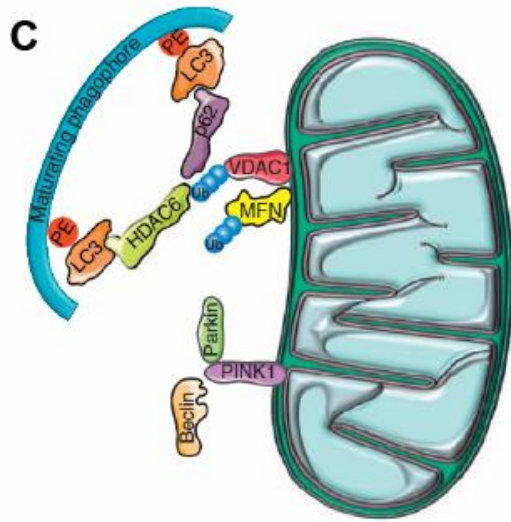

Fig. 2.10: Removal of damaged mitochondria via mitophagy.

(A) A reduction in the mitochondrial membrane potential results in stabilization of full-length PINK1 at the mitochondria which in turn recruits Parkin to the mitochondrial surface. (B) Parkin subsequently ubiquitinates OMM proteins like mitofusins, the mitochondrial fusion proteins, and VDAC1 resulting in recruitment of the adaptor proteins HDAC6 and p62. (C) The adaptors then bind to LC3 on pre-autophagosomal membranes thereby initiating mitophagy. Reprinted from Trends in Molecular Medicine, 17/3, Vives-Bauza and Przedborski, Mitophagy: the latest problem for Parkinson's disease, Pages 158-165, Copyright (2011), with permission from Elsevier. (VivesBauza and Przedborski, 2011).

Damaged mitochondria are then selectively targeted for mitophagy (as illustrated in Fig. 2.10), a process that is mediated by PINK1-dependent recruitment of Parkin to the mitochondrion [reviewed in (Springer and Kahle, 2011)]. While in healthy mitochondria, PINK1 is proteolytically processed and rapidly degraded, it accumulates on depolarized mitochondria because the cleavage is dependent on an intact mitochondrial membrane potential [reviewed in (Imai and Lu, 2011)]. Full length PINK1 subsequently recruits Parkin to the damaged organelle resulting in ubiquitination of OMM proteins like mitofusins and VDAC1 (Voltagedependent anion-selective channel protein 1). Ubiquitination of mitofusins stimulates their degradation thereby preventing fusion of the damaged mitochondrion with other organelles (Gegg and Schapira, 2011). Ubiquitination of proteins at the OMM leads to recruitment of several adaptor proteins which in turn bind LC3 on pre-autophagosomal membranes and thereby stimulate induction of mitophagy [reviewed in (Vives-Bauza and Przedborski, 2011)]. The fact that two PD-related proteins are key players in clearance of damaged mitochondria suggests that disturbance of mitophagy is an important pathway in PD pathogenesis. 


\subsection{Parkinson's Disease and Mitochondria}

A growing body of evidence supports the view that mitochondria play a key role in the pathogenesis of PD. In 1989, Schapira et al. reported that the activity of complex I of the respiratory chain is reduced in the SN of PD patients thereby providing the first hint that compromised mitochondrial function could be involved in the disease pathogenesis (Schapira et al., 1989). A number of subsequent studies demonstrated that the complex I deficiency in PD patients was not restricted to the SN but could also be seen in other tissues (Haas et al., 1995; Krige et al., 1992; Parker et al., 1989; Yoshino et al., 1992) suggesting that the pathological changes are more systemic and that it is rather the particular vulnerability of the SN to these defects which is causing the specific degeneration of neurons in this region (compare section 2.1.1 of this introduction). In line with reduced complex I activity being involved in the pathogenesis of PD, intoxication with MPTP (1-methyl-4-phenyl-1,2,3,6-tetrahydropyridine) was found to cause a parkinsonian syndrome. MPTP is metabolized to its active metabolite MPP + in astrocytes which is subsequently taken up by DA neurons via the DA transporter. In the neurons, it causes mitochondrial complex I inhibition resulting in reduced ATP production [reviewed in (Tipton and Singer, 1993)]. Epidemiological studies have further substantiated the finding that complex I inhibition contributes to the development of PD, as an increased risk for PD was reported following exposure to the pesticides rotenone and paraquat, which are known to be complex I inhibitors [reviewed in (Sherer et al., 2002)]. Besides reduced ATP levels, increased ROS production due to complex I inhibition has been proposed to contribute to neuronal death. This view is supported by several studies that have demonstrated increased oxidative damage and reduced activity of antioxidant systems in PD patients when compared to healthy controls [reviewed in (Henchcliffe and Beal, 2008)].

More recently, evidence for mitochondrial involvement in PD was also found in genetic models of the disease. Several genes that have been linked to PD are associated with mitochondria. Some of them encode for mitochondrial proteins (PINK1, HtrA2/Omi), some for proteins that can be translocated to mitochondria under certain conditions (Parkin, DJ-1) and others for proteins that have been shown to be at least partially associated to mitochondria ( $\alpha$ synuclein, LRRK2) [reviewed in (Bueler, 2009)]. Furthermore, it was reported that compromised function of these proteins (or overexpression in the case of $\alpha$-synuclein) results in impaired mitochondrial function ( $\alpha$-synuclein, DJ-1, PINK1, Parkin, HtrA2/Omi), changes in mitochondrial morphology (PINK1, Parkin, $\alpha$-synuclein, HtrA2/Omi) and increased ROS production or increased sensitivity to oxidative stress ( $\alpha$-synuclein, DJ-1, PINK1, Parkin) [reviewed in (Bueler, 2009; Burbulla et al., 2010)]. Therefore, multiple lines of evidence, ranging from toxin and genetic models of PD to examination of patient material, point towards mitochon- 
drial dysfunction being a key event in disease pathogenesis. Restoring mitochondrial function and morphology therefore seems to be a promising target for treatment of PD. But before this can be achieved, we need to gain a deeper understanding of the exact mechanisms causing mitochondrial dysfunction. Furthermore, it needs to be clarified whether mitochondrial defects are really present in all cases of PD or only in a subset while in others disturbance of the cellular degradation systems and accumulation of misfolded proteins is the predominant pathogenic pathway. 


\section{Aims}

A major challenge in the investigation of the molecular pathways involved in PD remains the identification of the early events that are involved in the initiation of the disease. This is particularly difficult as PD patients develop symptoms only after a substantial amount of DA neurons in the SN have already degenerated. Therefore, the establishment of model systems is important to study the early pathogenic mechanisms and will help to understand the physiological function of the PD-related genes. Determination of the disease-initiating mechanisms is essential for the development of novel therapeutic strategies aiming in preventing neuronal death in contrast to the currently used symptomatic treatments. Moreover, knowledge about early cellular changes will help to define biomarkers which are able to detect PD at early stages where a neuroprotective treatment would be most efficient.

As the cellular function and toxicity of many PD-related genes is still poorly understood, we decided to address these issues by using the model organism $C$. elegans. Due to its genetic tractability and the fact that worms are transparent, allowing for imaging in living animals, the nematode is an attractive system to examine the molecular mechanisms involved in PD pathogenesis. This study is focused on the investigation of two PD-related genes $\alpha$-synuclein $(\alpha S)$ and catp-6, the C. elegans ortholog of human ATP13A2(PARK9).

The first chapter of this dissertation is concerned with the cellular function and toxicity of aS. As $C$. elegans lacks an ortholog of this gene, transgenic worms expressing human aS were investigated. The aims for this part of the thesis were:

1) Determining whether fibrillar aggregates or rather soluble oligomeric precursors are responsible for toxicity of the protein. This aspect was addressed by comparing aggregation properties and toxicity of different variants of $\alpha S$ including synthetic variants that stop aggregation at the stage of soluble oligomers and do not form fibrils.

2) Investigation of the effects of $\alpha \mathrm{S}$ expression on mitochondrial morphology as several lines of evidence suggest that mitochondrial dysfunction plays an important role in PD pathogenesis. Besides wt $\alpha \mathrm{S}$, familial PD mutants of the protein and a synthetic prefibrillar variant were subjected to this analysis. 
Characterization of the lysosomal P-type ATPase CATP-6 was the second project of this dissertation and was done by examining deletion mutants of catp-6. The scopes for this project were as follows:

1) Phenotypic characterization of the deletion mutants (brood size, development, movement)

2) Investigation of the effects of CATP-6 loss-of-function on mitochondrial morphology

3) Determining whether mitochondrial function is impaired in catp- 6 deletion mutants

4) Study the effect of loss of function of the lysosomal P-type ATPase on autophagy 


\section{Chapter I: Cellular function and toxicity of $\alpha S$ in C. elegans}

\subsection{Introduction}

Aggregation of $\alpha S$ in Lewy bodies (LBs) is a very characteristic histological feature in the pathogenesis of PD (Baba et al., 1998; Spillantini et al., 1998; Spillantini et al., 1997; Takeda et al., 1998). Mutations in the aS gene (Kruger et al., 1998; Polymeropoulos et al., 1997; Zarranz et al., 2004) as well as duplication or triplication of the gene locus have been associated to familial forms of PD (Chartier-Harlin et al., 2004; Farrer et al., 2004; Ibanez et al., 2004; Singleton et al., 2003). The finding that multiplication of the $\alpha S$ gene was leading to PD was not only indicative of increased expression causing toxicity but moreover that the expression level was also correlated with severity of the disease, with higher aS expression leading to an earlier onset and rapid progression of the disease. More recently, $\alpha S$ has also been linked to sporadic forms of PD with changes in the promoter region leading to increased expression levels and representing a risk factor for the development of PD (Fuchs et al., 2008; Mueller et al., 2005; Pals et al., 2004) further confirming a dosage-dependent toxicity. Thus, aS might be the main agent causing PD. However, the mechanism causing toxicity is not yet clear.

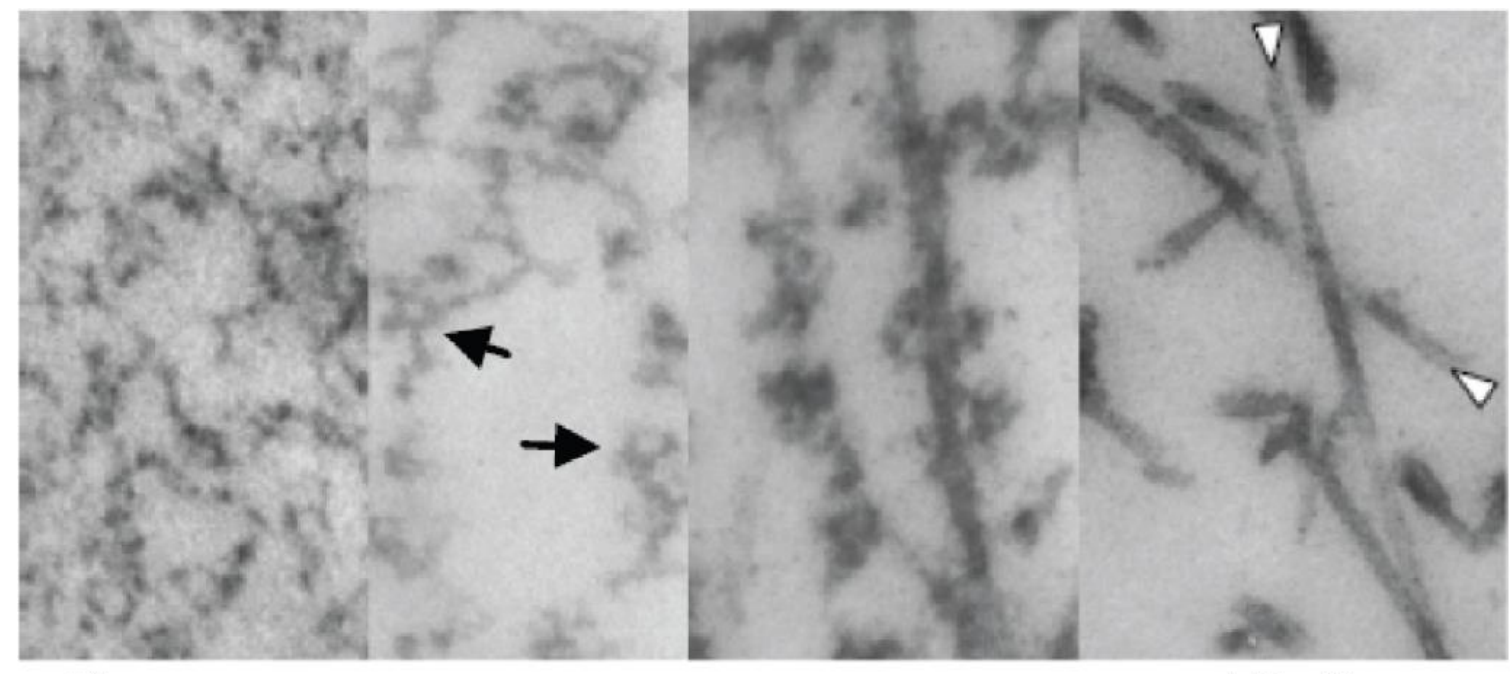

Monomers

Fibrils

Fig. 4.1: HPF-EM analysis of $\alpha \mathrm{S}$ aggregation in vitro.

The pictures represent the time course of $\alpha S$ aggregation after putting monomeric aS into refolding buffer. Solutions were frozen at different time points and EM images were taken. aS immediately starts to aggregate into small oligomers which assemble into proto-fibrils forming annular structures (black arrows). Later on, stable fibrils (white arrowheads) are formed. Pictures were kindly provided by Dr. Jan Hegerman. 
In vitro, aS rapidly aggregates to form amyloid fibrils. The process of aS aggregation in vitro, assessed by High-Pressure Freeze EM (HPF-EM), is shown in Fig. 4.1. When aS monomers are transferred to refolding buffer, they immediately start to aggregate. First, they assemble into small oligomeric precursors which are soluble and form annular structures. In the next step, the proto-fibrils start to assemble and further aggregate to fibrillar structures. In the end, clear fibrillar aggregates are observed. These fibrils are so-called paired helical filaments which are Thioflavin positive and which have also been observed in post-mortem brains of PD patients.

For a long time, the aggregated version of aS was held responsible for causing toxicity. This hypothesis was based on several observations: First, PD mutants of aS were shown to be more prone to aggregation than the wild type variant of the gene leading to increased occurrence of the aggregated aS in form of amyloid fibrils (Choi et al., 2004; Conway et al., 1998; Greenbaum et al., 2005; Li et al., 2001; Narhi et al., 1999). Second, aS fibrils were found to be the major constituent of LBs (Baba et al., 1998; Spillantini et al., 1998; Spillantini et al., 1997; Takeda et al., 1998). Third, increased expression levels of aS have been associated with PD and increased expression levels correlate with increased aggregation of the protein (Giasson et al., 1999; Uversky et al., 2001). However, it was not clear, whether indeed these amyloid fibrils are the toxic species or whether smaller oligomeric precursors of aS were responsible for the toxic effects. As proto-fibrils were reported to bind to membranes and form pores in the membranes, the question was raised whether these proto-fibrils might be the disease-causing agent of aS (Goldberg and Lansbury, 2000; Volles and Lansbury, 2003). Doubts against the hypothesis that aS fibrils are the pathogenic species were raised as in several animal models of aS overexpression, the rate of fibril formation did not correlate with the degree of toxicity (Auluck et al., 2002; Chen and Feany, 2005). It was even speculated that the fibrillar aggregates might be protective for the cell as they might be less reactive and lead to a reduced surface being in contact with the cellular components (Chen and Feany, 2005).

To identify the toxic species of $\alpha S$, we used different variants of aS with altered aggregation properties. This project was performed in collaboration with Prof. Markus Zweckstetter (MPI for Biophysical Chemistry, Göttingen, Department for Protein Structure Determination Using NMR) and Prof. Christian Griesinger (MPI for Biophysical Chemistry, Göttingen, Department for NMR Based Structural Biology) who designed synthetic variants of aS that stop aggregation at the stage of proto-fibrils and do not form amyloid fibrils. The design of these prefibrillar variants of $\alpha S$ was based on the fact that $\alpha S$ aggregation is dependent on the formation of $\beta$-sheets. As the regions involved in $\beta$-sheet formation are known, one can try to 
prevent this by mutating essential residues in these areas. Proline residues are known to be $\beta$-breaking because they are not able to form hydrogen bonds via their backbone $\mathrm{NH}$-group. Thus, the synthetic pre-fibrillar variants were generated by introducing proline residues at positions of the protein that are involved in $\beta$-sheet formation.

We used the nematode $C$. elegans as a model to study aggregation and toxicity of the different $\alpha \mathrm{S}$ variants in vivo. $C$. elegans has previously been used for both genetic and toxin models of PD. It has to be noted that worms do not have an ortholog of $\alpha S$ but worms expressing human wt aS or the PD mutants thereof have been used successfully before to study toxicity of this protein in the nematode (Cao et al., 2005; Kuwahara et al., 2006; Lakso et al., 2003; Springer et al., 2005; Ved et al., 2005). Furthermore, aggregation of heterologously expressed $\alpha \mathrm{S}$ in body wall muscle (BWM) cells of $C$. elegans has been assessed in other studies (Gitler et al., 2009; Hamamichi et al., 2008; van Ham et al., 2008).

\subsection{Results}

\subsubsection{Generation of synthetic aS variants that are not forming fibrils in vitro}

In order to assess whether fibrillar aggregates or rather smaller oligomeric variants are responsible for $\alpha S$ induced toxicity, five different $\alpha S$ variants with different propensity of forming fibrillar aggregates were used. Besides wild type aS, two PD mutants (A30P and A53T) exhibiting increased aggregation and two synthetic variants (A56P and TP) that were designed to impair aggregation, were used.

The synthetic variants were designed by the group of Prof. Markus Zweckstetter (MPI for Biophysical Chemistry, Göttingen, Department for Protein Structure Determination using NMR) by introducing proline residues at positions of the protein that are part of the $\beta$-sheet rich core which is essential for the formation of highly ordered amyloid fibrils (Karpinar et al., 2009). The synthetic construct A56P was generated by introducing just one proline mutation whereas the TP (triple proline) variant is characterized by three proline mutations (A30P, A56P and A76P). The positions of the different mutations of the PD and synthetic variants of aS are indicated in Fig. 4.2A. 
A

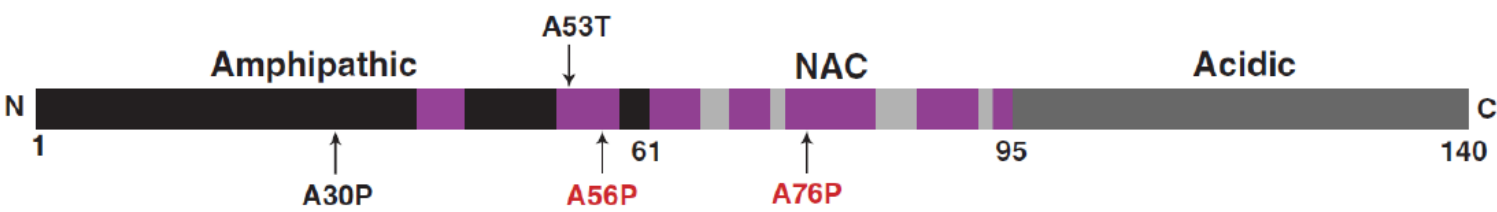

B

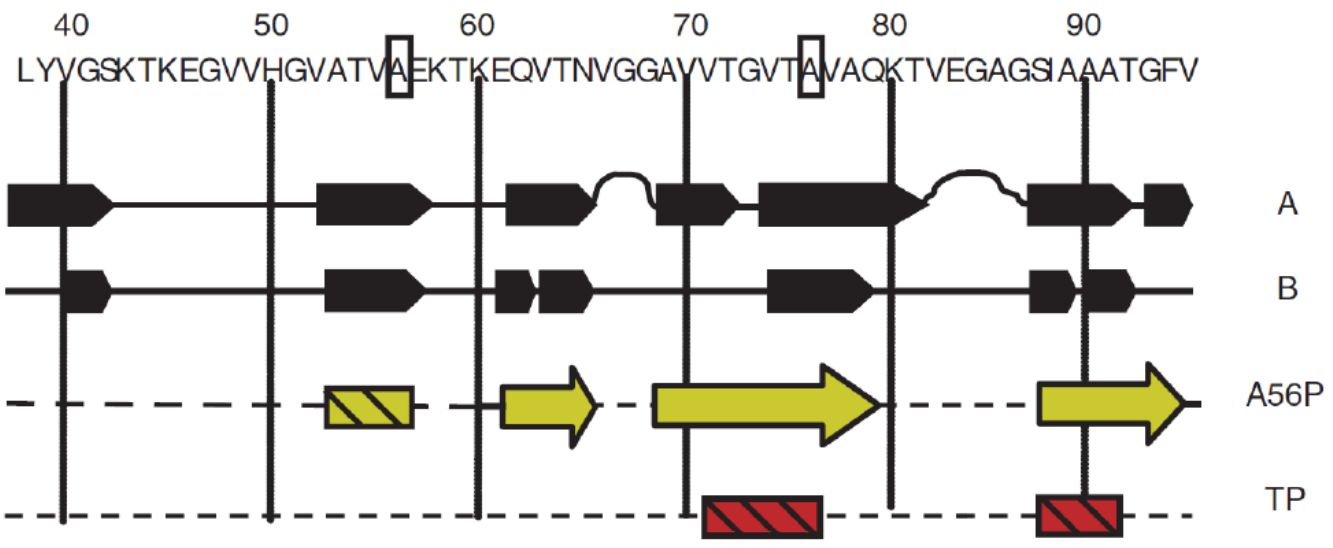

C

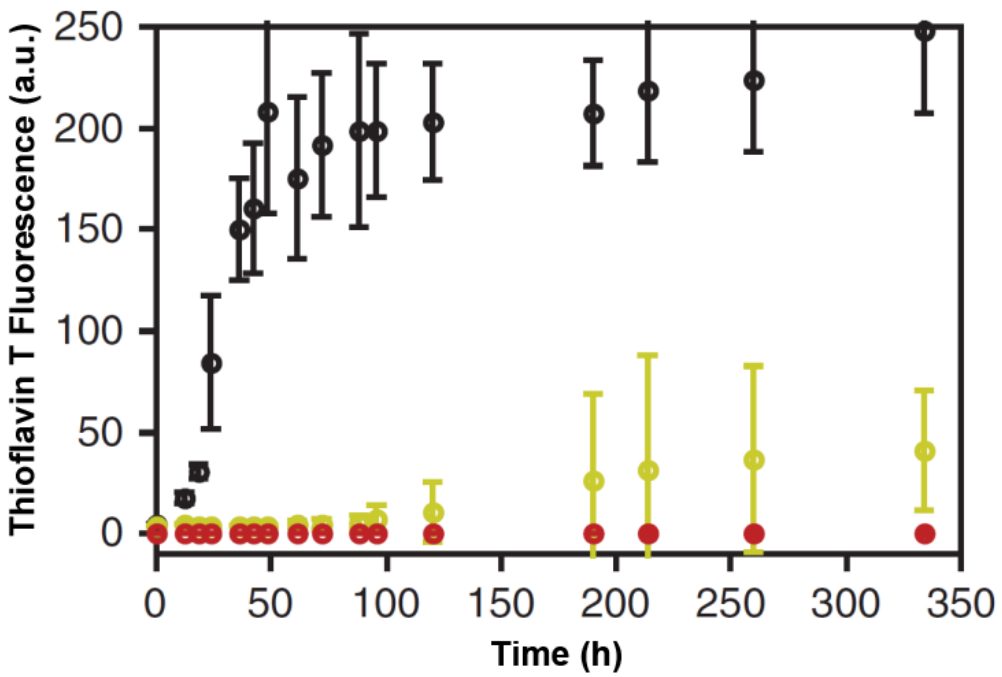

- wt aS

- A56P aS

- TP aS

Fig. 4.2: Synthetic variants of aS exhibit decreased rates of fibril formation in vitro because of impaired $\beta$-sheet structures.

(A) Positions of mutations of the different variants used in this study are indicated in black for the PD mutations and in red for the designed mutations. Parts of the protein that are involved in the formation of $\beta$-sheets (Heise et al., 2005) are shown in purple. Both designed proline residues were introduced at positions that are part of the $\beta$ sheet-rich core of $\alpha \mathrm{S}$. (B) Representation of the $\beta$-sheets formed by the different variants of $\alpha \mathrm{S}$. Fully formed $\beta$ sheets are indicated as arrows. Impaired $\beta$-sheet structures are depicted as striped boxes. Two different $\beta$-sheet structures of wt $\alpha \mathrm{S}$ are indicated in black. In A56P (shown in yellow) only the $\beta$-sheets in the N-terminal part of the protein are affected while in TP (depicted in red) also C-terminal $\beta$-sheets are impaired. (C) Formation of amyloid fibrils was assessed by measuring Thioflavin T fluorescence. While wt $\alpha \mathrm{S}$ (black) rapidly aggregates into fibrillar aggregates after a short lag-phase, fibril formation is severely impaired in A56P (yellow) and absent in TP aS (red). Pictures were published in (Karpinar et al., 2009) and were kindly provided by Prof. Markus Zweckstetter. 
While in A56P only the $\beta$-sheet structures in the N-terminal part are affected, the TP variant shows severe impairment of $\beta$-sheet formation throughout the entire protein (Fig. 4.2B) (Karpinar et al., 2009). The formation of amyloid fibrils was severely impaired in the designed variants of $\alpha S$ as seen by the absence of Thioflavin fluorescence for TP $\alpha S$ and the strong decrease in fluorescence for A56P (Fig. 4.2C) (Karpinar et al., 2009). Wt aS rapidly starts forming fibrils after a short lag-phase. In contrast, the onset of fibrillization strongly delayed for A56P aS and was not seen within the monitored 300 min for TP aS and even not after two weeks of incubation. However, occurrence of amyloid fibrils could be observed after long incubation times of very high concentrations of TP $\alpha S$ indicating that the formed oligomers are able to further aggregate and only the rate of fibril formation is tremendously decreased (Karpinar et al., 2009). NMR analysis was performed by the laboratory of Prof. Markus Zweckstetter to investigate whether the designed aS variants were still aggregating to the oligomeric precursors of the fibrils and indeed, the proto-fibrils were still found for A56P and TP (Karpinar et al., 2009).

Thus, in vitro examination of the synthetic aS variants clearly showed that they stop aggregation at the stage of soluble oligomers and that they are not forming amyloid fibrils. Therefore, these pre-fibrillar variants are well-suited for analyzing whether fibrils or oligomeric precursors are the toxic species of $\alpha \mathrm{S}$.

\subsubsection{TP aS prevents fibril formation in C. elegans}

Before testing the neurotoxicity of the different aS constructs, we wanted to confirm in vivo, that the fibril formation was indeed absent for the designed variants. For assessing aggregation of aS in C. elegans, an aS-construct that is tagged with mYFP at the C-terminus was created. C-terminal fusion of YFP or GFP to aS has been done before (Gitler et al., 2009; Hamamichi et al., 2008; Rockenstein et al., 2005; van Ham et al., 2008) and it was recently shown that the tag doesn't interfere with aggregation (van Ham et al., 2010). The aS-mYFP construct was expressed in $C$. elegans under the control of the body wall and sex musclespecific myo-3 promoter. Occurrence of fibrillar aggregates was assessed in the vulva muscles as those cells were exhibiting high expression levels and they were easily recognized in every worm, so that it could be made sure that always the same cells were investigated. As augmented aggregation was reported for aged worms, 10 day old animals were examined. Representative pictures of the $\alpha S$ expressing vulva muscles for wt and TP $\alpha S$ are shown in Fig. 4.3A+B, respectively. Fibrillar aggregates were seen in the vulva musculature of around $80 \%$ of the worms from two independent transgenic lines expressing the wt protein. In contrast, aggregates of TP were almost completely lacking with less than $10 \%$ of the worms 
showing aggregates in two independent transgenic lines (Fig. 4.3C). If aggregates were found in the TP expressing animals, they were much smaller and the number of aggregates per cell was also diminished. Expression levels of wt and TP aS were determined by Western Blot as shown in Fig. 4.3D. The fact that TP aS expression was even slightly higher than that of wt aS eliminated the possibility that lower concentration of the protein would account for the reduced aggregation.
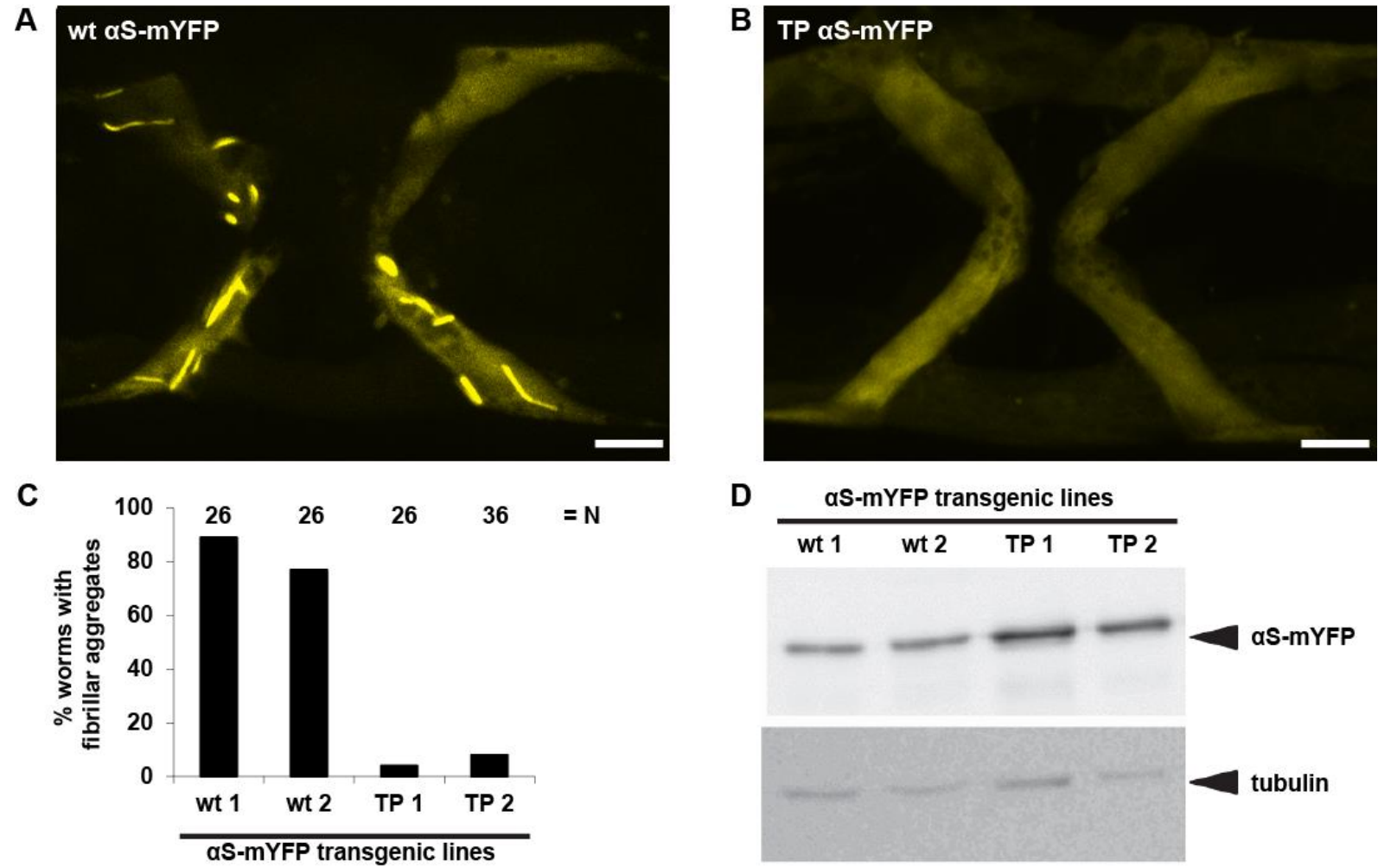

Fig. 4.3: Formation of fibrillar aggregates is absent in $C$. elegans muscle cells expressing TP $\alpha S$.

$(A+B)$ Vulva musculature of 10 day old worms expressing pmyo-3::wt $\alpha S-m Y F P$ or pmyo-3::TP $\alpha S-m Y F P$, respectively. Scale bars represent $10 \mu \mathrm{m}$. While numerous fibrillar aggregates are found in worms expressing wt aS $(A)$, those aggregates are absent when TP aS is expressed (B). (C) Quantification of the occurrence of fibrillar aggregates in the vulva muscles of wt or TP aS expressing worms for two independent transgenic lines each.

(D) Western blot was performed to confirm that expression levels of wt and TP aS were comparable. Pictures were published in (Karpinar et al., 2009) and are shown with slight modifications.

\subsubsection{TP $\alpha \mathrm{S}$ exhibits increased neurotoxicity in $C$. elegans}

After having verified that the designed TP $\alpha \mathrm{S}$ mutant does not form fibrillar aggregates when expressed in C. elegans, we wanted to compare the toxicity of wt and PD mutant $\alpha S$ with that of the designed pre-fibrillar TP variant in vivo. If, as hypothesized, soluble oligomers of $\alpha S$ are the toxic species, one would expect that TP $\alpha$, which forms high levels of these oligomers but no fibrillar aggregates, would exhibit the highest toxicity. If, in contrast, amyloid fibrils are the main agent causing toxicity, the PD mutants should exhibit the highest degree of 
toxicity and TP $\alpha$ S, that is not forming fibrils, should be much less toxic. Thus, comparison of the toxicity of the different variants of $\alpha \mathrm{S}$ in vivo will help to elucidate whether fibrils or rather soluble oligomeric precursors are the toxic species of aS. The experiments described in this section were done by Dr. Stefan Eimer.

Dopaminergic neuron degeneration is a well-established model for determining neurotoxicity in C. elegans (Benedetto et al., 2010; Braungart et al., 2004; Kuwahara et al., 2006; Lakso et al., 2003; Locke et al., 2008; Nass et al., 2002) and was therefore chosen to assess the neurotoxic effects of the different constructs. $C$. elegans hermaphrodites have eight DA neurons (Sulston et al., 1975) of which six are located in the head of the animal (ADE and CEP neurons). Visualization of the DA neurons is achieved by expression of mRFP under the control of the DA neuron-specific dat-1 (DopAmine Transporter) promoter. Morphology of the six head DA neurons is shown in Fig. 4.4A. The different aS mutants were also expressed under the control of a dat-1 promoter. Moderate expression levels of aS were chosen, so that no effect on DA neuron morphology was seen in the worms expressing wt aS (Fig. 4.4A, top panel). In contrast, dopamine neuron degeneration was observed in DA neurons of worms expressing different mutants of aS (Fig. 4.4A, lower panel). The extent of degeneration caused by the different variants of $\alpha S$ was quantified by determining the percentage of worms that were not showing defects in neurite morphology of DA neurons in the head and the result is visualized in Fig. 4.4B. Unlike expression of wt $\alpha \mathrm{S}$, which leads to almost no neurite defects, the PD mutants A30P and A53T are causing degeneration in $40-50 \%$ of the worms. Remarkably, the synthetic variants A56P and especially TP are showing even more severe defects than the PD mutants with $60 \%$ and $80 \%$ of the animals manifesting defective neurites.

We further investigated whether the observed defects in neurite morphology correlate with impaired function of these neurons. On a molecular level, the dopaminergic system of worms strongly resembles that of humans with dopamine transporters, receptors and metabolic pathways being highly conserved [reviewed in (Nass and Blakely, 2003)]. In humans, dopamine signaling has been implicated in the coordination of movement and the recognition of reward. DA signaling in $C$ elegans plays a role in egg-laying, defecation, the modulation of motor activity, response to food sources and in the habituation to touch [reviewed in (McDonald et al., 2006)]. Thus, also on a functional level, similarities between the dopaminergic systems of worms and humans exist. The food slowing response is a well-studied DAdependent behavior in C. elegans which can easily be assessed (Kuwahara et al., 2006; Sawin et al., 2000). Worms slow down their locomotion and switch from a food searching to a feeding behavior as soon as they encounter a food source. By assessing the speed of worms 
in the absence and in the presence of food, one can determine the food slowing response. If the function of DA neurons is impaired, the worms slow down to a lesser degree when encountering food. Consistent with the results of the morphological defects, the slowing rate in response to food was most severely impaired in worms expressing TP $\alpha \mathrm{S}$. The fact that the pre-fibrillar variants of aS exhibit highest neurotoxicity clearly points towards oligomeric precursors and not the amyloid fibrils being the toxic species of $\alpha \mathrm{S}$.

A

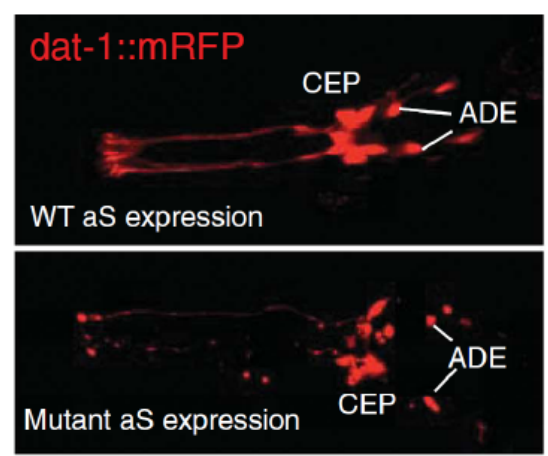

B

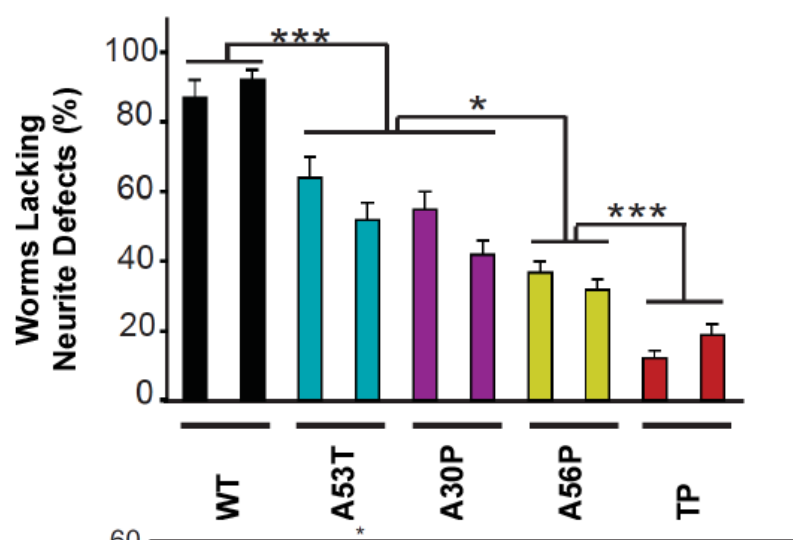

C

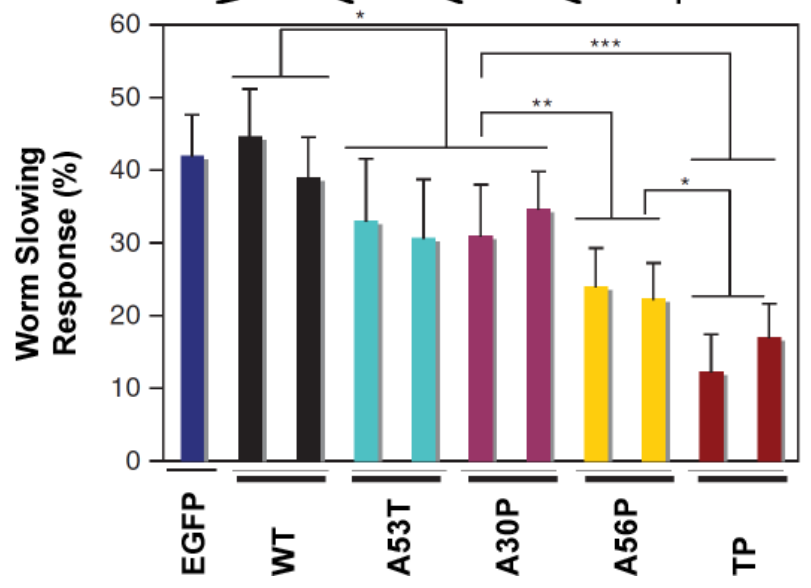

Fig. 4.4: Neurotoxicity is increased in the pre-fibrillar variant TP aS.

(A) Morphology of DA neurons in the $C$. elegans head was visualized by expressing mRFP under the control of the DA neuron-specific dat-1 promoter. While moderate expression of wt aS does not produce neurite defects (top panel), expression of different mutants leads to neurite degeneration (lower panel). (B) Quantification of the degree of degeneration in worms expressing different variants of aS. Most neurite defects were observed in the designed variant TP $\alpha$ S. Two independent transgenic lines were examined for each variant and neurites of 78-80 animals were analyzed. The error bars correspond to the standard error of the mean (SEM) and the significance values of the ANOVA test are indicated: ${ }^{*} P<0.05$; ${ }^{* \star *} P<0.001$. (C) $D A$ neuron function was analyzed by measuring the food slowing response of the worms. In accordance with the morphological defects, this DA-dependent behavior was most severely affected in the pre-fibrillar aS variants. For each aS variant expressed, at least two independent transgenic lines were tested ( $N=40-50$ animals per trial, 3 trails). The slowing rate corresponds to the average decrease in movement (body bends per min) for animals placed in food as compared with animals without food. Animals expressing only EGFP in dopaminergic neurons are shown as control. The errorbars correspond to SEM and the significance values of the ANOVA test are indicated: ${ }^{*} \mathrm{P}<0.05 ;{ }^{* *} \mathrm{P}<0.01 ;{ }^{* * *} \mathrm{P}<0.001$. Pictures were published in (Karpinar et al., 2009) and were kindly provided by Dr. Stefan Eimer. 


\subsubsection{Expression of $\alpha \mathrm{S}$ leads to drastic changes of mitochondrial morphology in C. elegans muscle cells}

After having identified pre-fibrillar oligomers as toxic species of $\alpha \mathrm{S}$, the next step of my PhD project was to investigate the cellular mechanisms by which aS causes toxicity. As recently increasing evidence for the involvement of mitochondria in PD pathogenesis and other neurodegenerative diseases was reported, we focused our studies on this cellular compartment. aS has been shown to bind to cellular membranes including the mitochondrial membrane (Chinta et al., 2010) and mitochondrial pathology as well as increased oxidative stress was described in several models upon overexpression of aS (Hsu et al., 2000; Lee et al., 2001; Martin et al., 2006; Poon et al., 2005).

Therefore, we decided to examine the morphology of mitochondria in $C$. elegans muscle cells by fluorescence microscopy making use of TOM70-CFP (transmembrane domain of yeast TOM70 fused to CFP; (Labrousse et al., 1999); plasmid kindly provided by Alexander van der Bliek, UCLA), a marker for the mitochondrial outer membrane expressed under the control of a muscle-specific myo-3 promoter. Reasons for choosing BWM cells for the analysis of mitochondrial morphology were the large size of these cells compared to other cell types $-C$. elegans muscle cells measure $\sim 100 \mu \mathrm{m}$ in length with a width of $\sim 10 \mu \mathrm{m}$ while neuronal cell bodies measure 3-5 $\mu \mathrm{m}$ in diameter - and because BWM cells show a very regular and stereotyped arrangement of mitochondria.

The tubular morphology of mitochondria in wt worms that are not expressing $\alpha \mathrm{S}$ is depicted in Fig. 4.5A. The nucleus of muscle cells can easily be identified due to the absence of mitochondria in this area. Upon expression of wt $\alpha \mathrm{S}$, two different changes in mitochondrial morphology were observed. In some cells, mitochondria become fragmented forming vesicular mitochondria (Fig. 4.5B) that differ in diameter between cells. In other cells, the occurrence of very thin and elongated mitochondria was observed (Fig. $4.5 \mathrm{C}$ ). Notably, the thin mitochondria seen in muscle cells expressing aS also form more interconnected networks. While in mitochondria of wild type worms the luminal space inside the stained mitochondrial outer membranes is seen, it is not visible in the thin and elongated mitochondria.

The effect of aS on mitochondrial morphology in $C$. elegans body wall muscles (BWMs) is concentration-dependent as shown in the quantification of mitochondrial morphology upon expression of aS in different concentrations depicted in Fig. 4.5D. Low expression levels of aS lead to the occurrence of fragmented and thin mitochondria in only $10-30 \%$ and $5-15 \%$ of muscle cells, respectively while $55-80 \%$ of the muscles are still showing wt-like morphology. In contrast, only around $15 \%$ of the cells exhibit wild-type like mitochondrial tubules for the 
worms expressing high concentrations of aS. In those animals, approximately $40 \%$ of the cells are showing mitochondrial fragmentation and another $40 \%$ of the cells have very thin and elongated mitochondria. Saturation of the effects can be observed as increasing expression levels above a certain level does not lead to aggravation of the observed morphological changes. This suggests that mitochondrial morphology is affected by the expression of aS and that the severity of the effects seen, like mitochondrial fragmentation and occurrence of long and thin mitochondria, is dependent on aS expression levels.
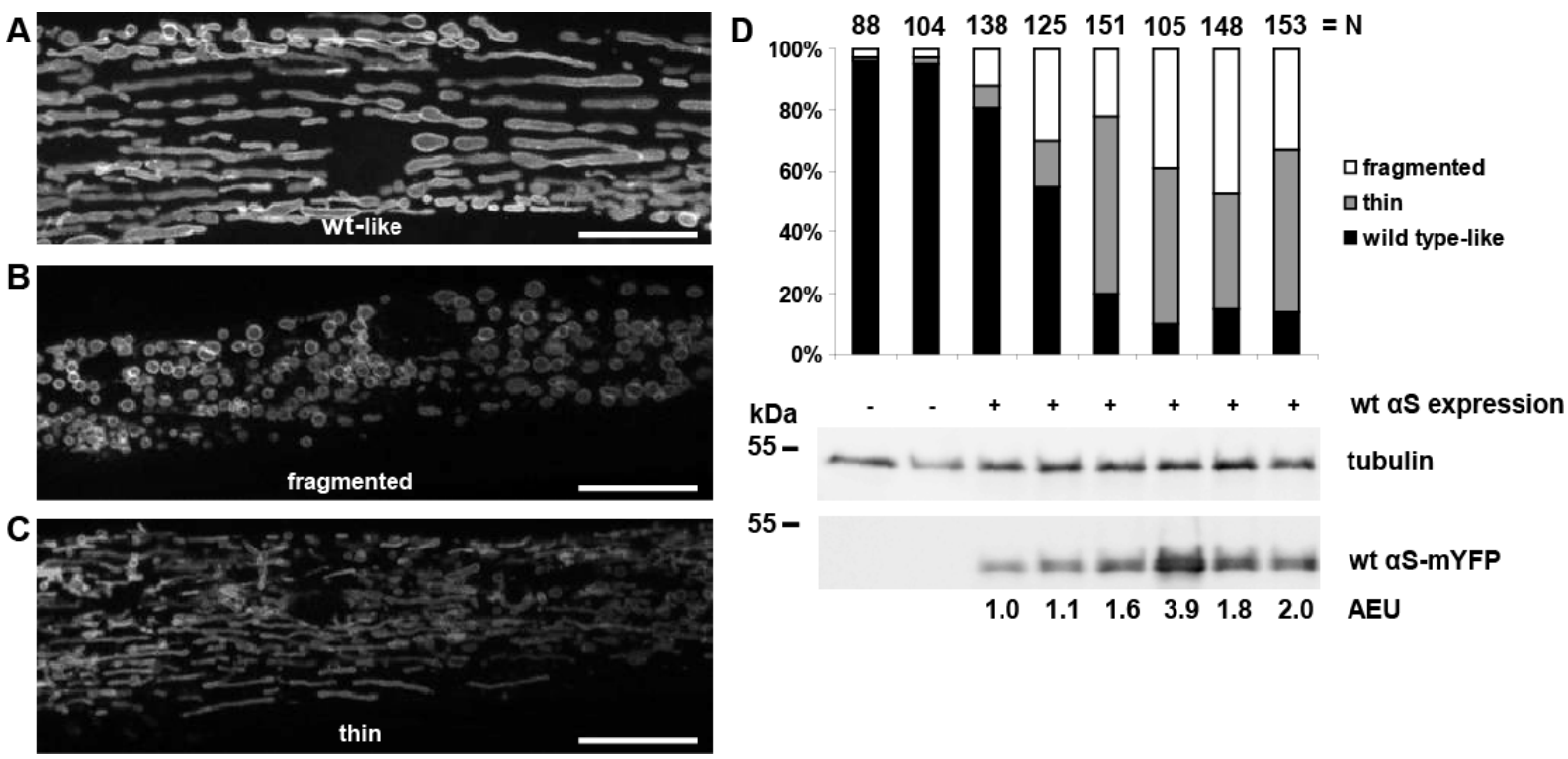

Fig. 4.5: Changes in mitochondrial morphology in C. elegans muscle cells expressing aS.

(A-C) Mitochondrial morphology in $C$. elegans muscle cells was assessed by expression of aS together with the mitochondrial outer membrane marker TOM70-CFP (transmembrane domain of yeast TOM70 fused to CFP) under control of the muscle-specific myo-3 promoter. Scale bars represent $10 \mu \mathrm{m}$. (A) Mitochondria in wt worms that are not expressing aS form regular tubular structures. Upon expression of wt aS, the mitochondria become either fragmented (B) or for very thin, elongated and interconnected structures (C). (D) Quantification of the effect of different concentrations of wt aS on mitochondrial morphology in $C$. elegans muscle cells. Expression levels of aS were determined by Western Blot. Pictures A-C were published in (Kamp et al., 2010).

We next wanted to confirm the mitochondrial morphology defects on an ultra-structural level using High Pressure Freeze Electron Microscopy (HPF-EM). Indeed, EM analysis confirmed the effects seen with fluorescence microscopy, especially the mitochondrial fragmentation. As only single cross-sections of BWM cells were analyzed by EM, it was hard to detect the occurrence of long and thin mitochondria as one would expect them to look similar to fragmented mitochondria in cross-sections. A decrease in mitochondrial diameter could clearly be observed in aS expressing worms as shown in Fig. 4.6. In contrast, no significant changes of the mitochondrial cristae structure could be observed. 

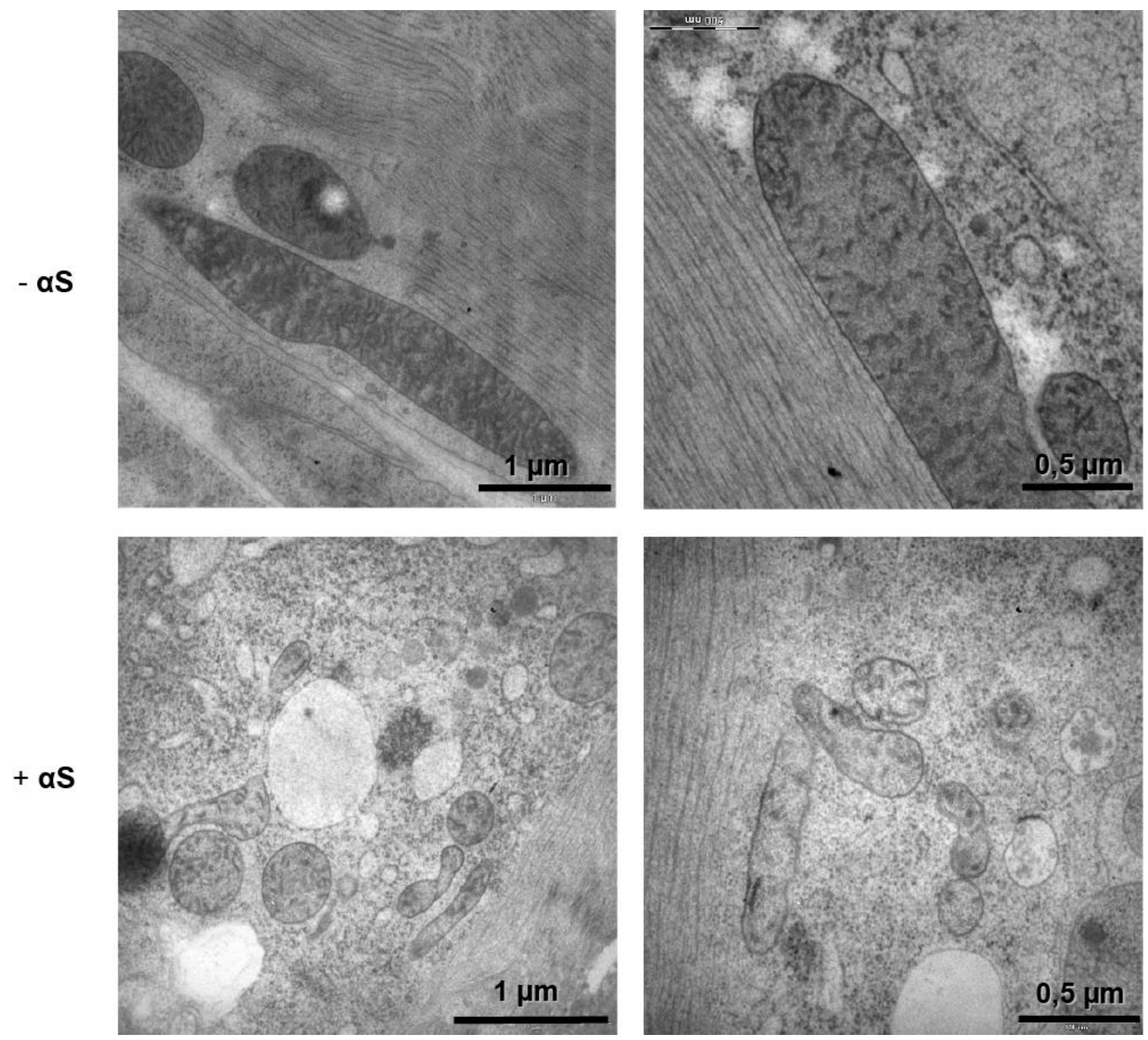

Fig. 4.6: EM analysis of changes in mitochondrial morphology in C. elegans muscle cells induced by expression of wt aS.

EM images of $C$. elegans BWM cells. The top images were taken from wild type animals that were not expressing aS, while the images in the bottom show BWMs of animals expressing wt $\alpha \mathrm{S}$ at relatively high expression levels. Mitochondrial fragmentation is seen in the worms that are expressing aS confirming the results obtained by fluorescence microscopy. EM images were kindly provided by Dr. Jan Hegerman.

The next step after investigation of the effects of wt aS was to determine the influence of PD mutants as well as the designed pre-fibrillar variant TP $\alpha$ S on mitochondrial morphology. The results of this analysis are illustrated in Fig. 4.7A. Interestingly, the synthetic TP aS, which stops aggregation at the stage of soluble proto-fibrils (see section 4.2.1) and exhibits increased levels of toxicity (see section 4.2.2), predominantly leads to the occurrence of long and very thin mitochondria and not so much to mitochondrial fragmentation. Only $10-30 \%$ of the cells expressing TP aS show vesicular mitochondrial structures while mitochondria in $30-50 \%$ of the cells expressing the wt version of the protein are fragmented. The number of cells with wt-like mitochondria is comparable in worms expressing wt and TP aS. 
The two PD mutants of $\alpha \mathrm{S}, \mathrm{A30P}$ and A53T, had similar effects on mitochondrial morphology than wt $\alpha \mathrm{S}$. The effect of A30P $\alpha \mathrm{S}$ was even lower than that of wt $\alpha \mathrm{S}$ although the expression levels were higher for A30P. It has to be noted at this point that the expression levels of TP aS observed for three independent transgenic lines were considerably higher than those for wt aS which might partially explain the different effects. Nevertheless, as we observed a saturation of the effects on mitochondrial morphology at the highest expression levels of our wt aS expressing worms, it seems rather unlikely that the discrepancy of effects seen in TP and wt $\alpha S$ is solely due to different protein concentrations in the cell. This is further supported by the fact that expression levels of A30P were similar to those of TP aS and still did not cause such severe defects on the appearance of mitochondria. We were trying to achieve similar expression levels of the different $\alpha S$ variants by injecting the same plasmid concentrations for creating transgenic lines but we still saw considerable differences in the actual expression levels in our transgenic lines. In order to be able to fully exclude that the observed differences of mitochondrial morphologies are due to dissimilar protein concentrations, more lines expressing the different variants at various concentrations would need to be generated and analyzed for mitochondrial morphologies.

EM analysis of the TP aS expressing worms confirmed the changes in mitochondrial morphology towards thinner diameter that was seen in the fluorescence pictures as shown in Fig. 4.7B. Again, the mitochondrial cristae structure was not impaired by expression of TP $\alpha \mathrm{S}$ similar to what was already observed for expression of wt $a \mathrm{~S}$.

In summary, expression of wt as well as the PD mutant A53T aS leads to mitochondrial fragmentation and the occurrence of muscle cells with long and thin mitochondria. The other PD variant, $\mathrm{A} 30 \mathrm{P}$, seems to affect mitochondrial morphology to a lesser extent. Interestingly, expression of the pre-fibrillar variant TP $\alpha \mathrm{S}$, which was shown to exhibit increased neurotoxicity, predominantly leads to long and thin mitochondrial morphology whereas mitochondrial fragmentation can only be seen to a small extent. 


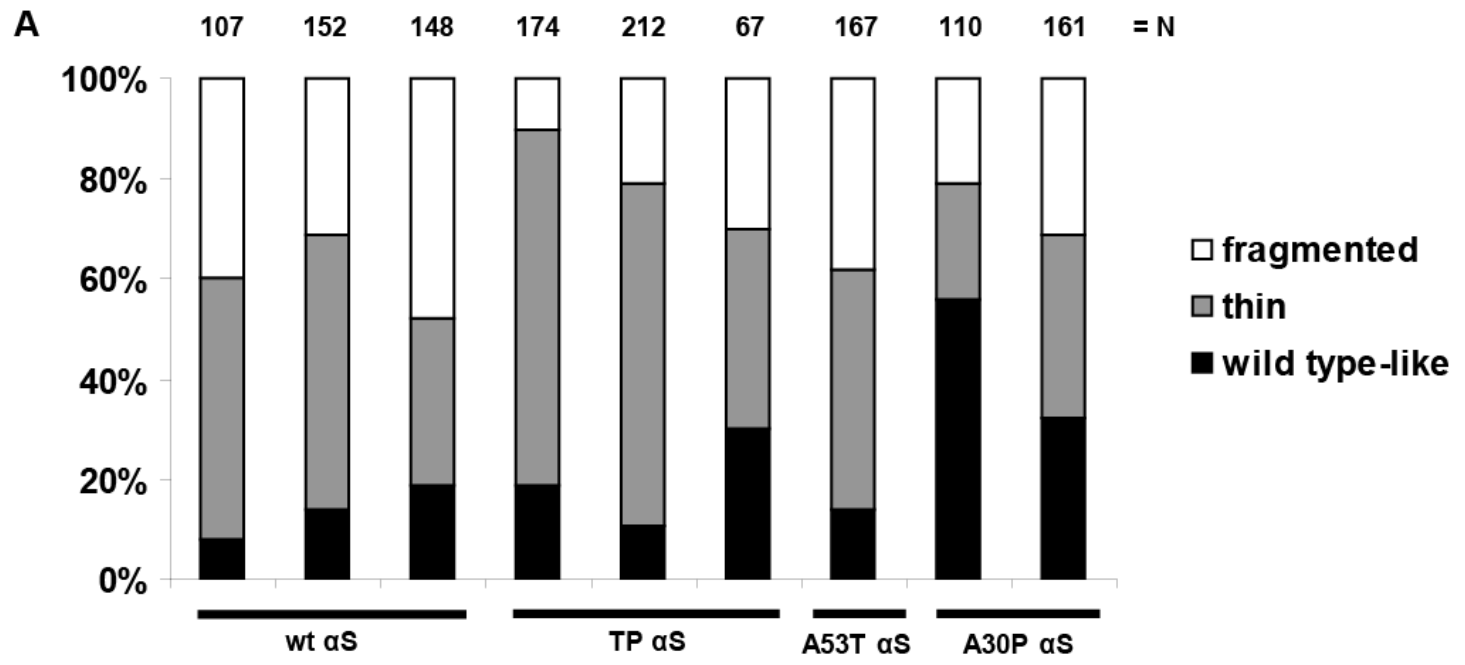

$\mathrm{kDa}$

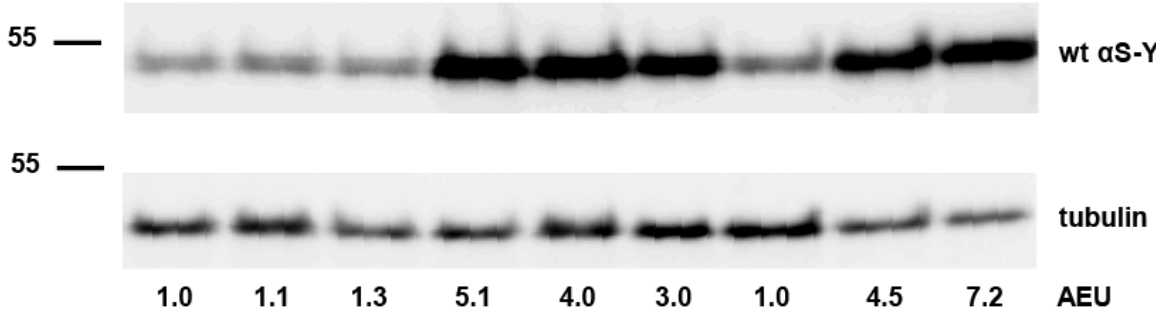

B

Wild type

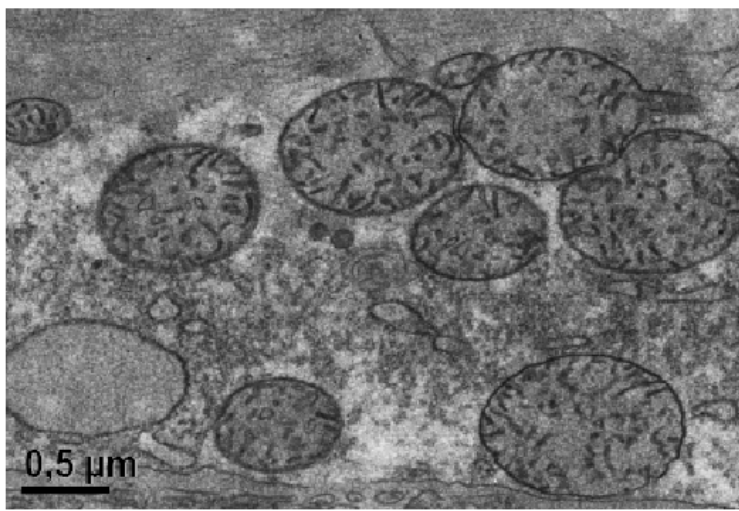

TP as

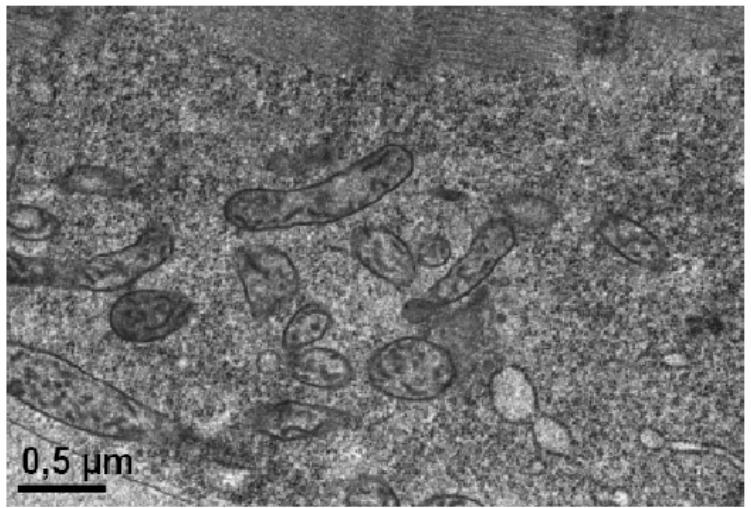

Fig. 4.7: Effect of different aS variants on mitochondrial morphology in C. elegans BWM cells.

(A) Quantification of the relative occurrence of wt-like, fragmented or thin mitochondria. TP aS leads to increased occurrence of long, thin and interconnected mitochondria while the PD mutants cause similar or even milder defects than those seen upon expression of wt aS. Expression levels of the different variants were determined by Western Blot. Three independent transgenic lines were analyzed for wt and TP aS, two for A30P and one for A53T. (B) EM pictures of cross-sections through C. elegans BWM cells. The diameter of mitochondria in cells expressing TP $\alpha \mathrm{S}$ is severely decreased compared to wild type worms not expressing aS. EM Images were kindly provided by Dr. Jan Hegerman. 


\subsubsection{Mitochondrial fragmentation similar to that seen upon expression of aS also occurs during aging}

Seeing the drastic changes in mitochondrial morphology upon expression of aS, we were wondering whether similar morphological alterations could be found in aged worms. In order to address this question, mitochondrial morphology in 3-day old (first day of adulthood) and 7-day-old animals (fourth day of adulthood in C. elegans is already at a post-reproductive stage) was investigated. This was done in both wild type worms not expressing aS and worms expressing wt $\alpha \mathrm{S}$. Fig. 4.8B depicts the result of this analysis. Interestingly, in the old wild type animals, a significant number of cells exhibited mitochondrial fragmentation with vesicular mitochondrial structures similar to those observed in young adult worms expressing wt $\alpha \mathrm{S}$ (Fig. 4.8A). In contrast, aging had only a minor impact on mitochondrial morphology in worms expressing aS. This suggests that the mitochondrial fragmentation observed upon expression of $\alpha \mathrm{S}$ is not an unphysiological reaction to the heterologous expression of this protein but rather a condition that is also found in aged nematodes. This points towards aS accelerating the physiological mitochondrial aging process.
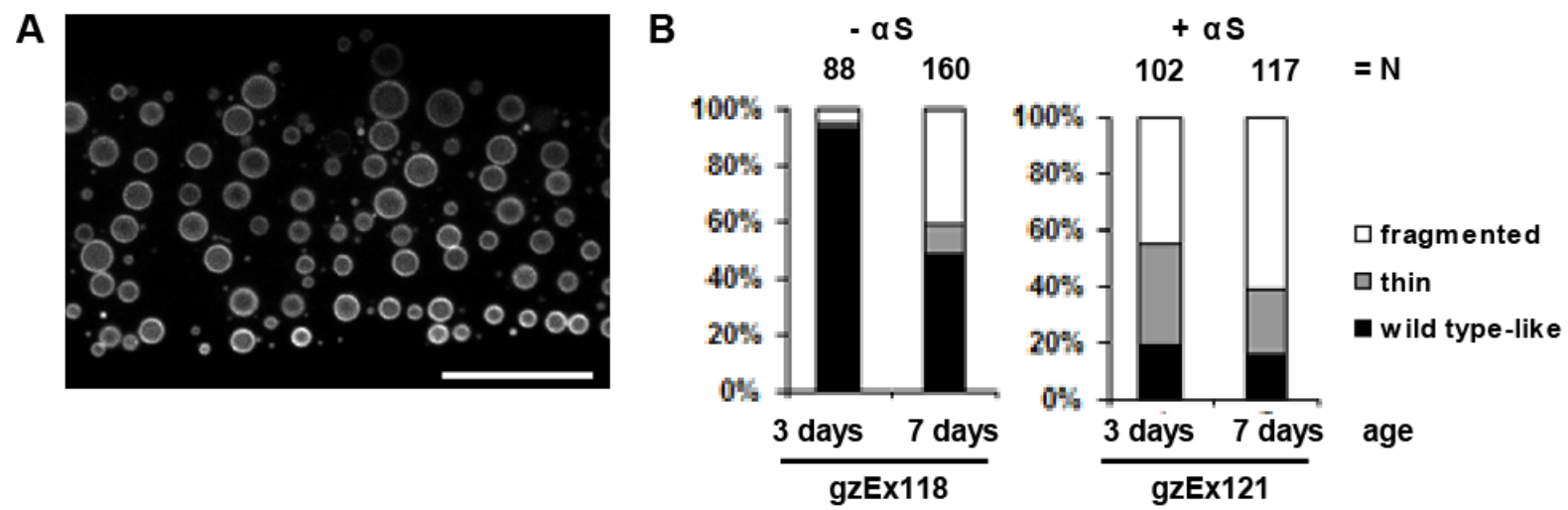

Fig. 4.8: Mitochondrial fragmentation in C. elegans muscle cells during aging.

(A) Fragmented mitochondria are found in BWM cells of 7 day-old wild type $C$. elegans. Scale bar represents $10 \mu \mathrm{m}$. (B) Quantification of mitochondrial morphology in young versus old worms for wild type worms (left) and worms expressing wt aS (right). In wild type worms not expressing $\alpha \mathrm{S}$, a considerable increase in fragmented mitochondria was seen during aging. In contrast, only mild changes in mitochondrial morphology were seen in aged aS expressing worms compared to young worms expressing aS. Pictures were published in (Kamp et al., 2010). 


\subsubsection{Changes in mitochondrial morphology caused by expression of aS re- semble those seen under starvation}

Besides checking for the influence of aging, we were also investigating how the nutrient status of cells might influence mitochondrial morphology. It was recently described that nutrient deprivation leads to mitochondrial elongation. This elongation is mediated by CAMP-induced activity of PKA (protein kinase A) which phosphorylates the mitochondrial fission protein DRP-1 (dynamin related protein 1) at Ser637 thereby preventing translocation of DRP-1 to the mitochondria. The result is unopposed fusion and thus elongation of the mitochondria (Gomes et al., 2011). The authors propose that mitochondrial elongation serves as a mechanism to prevent mitochondria from being cleared by autophagy which is induced upon nutrient depletion. Furthermore, they show that the elongation is accompanied by an increase of inner membrane cristae leading to increased ATP production. If mitochondrial elongation during starvation is prevented, mitochondria in contrast consume cytosolic ATP resulting in a bioenergetics crisis and eventually in cell death. Inhibition of mitochondrial elongation during starvation furthermore results in increased degradation of mitochondria via autophagy.

unstarved

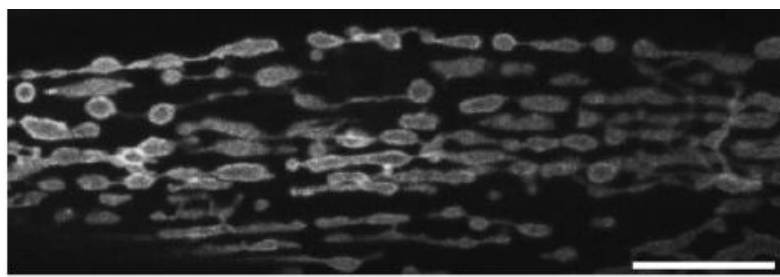

starved 24h starting from L4

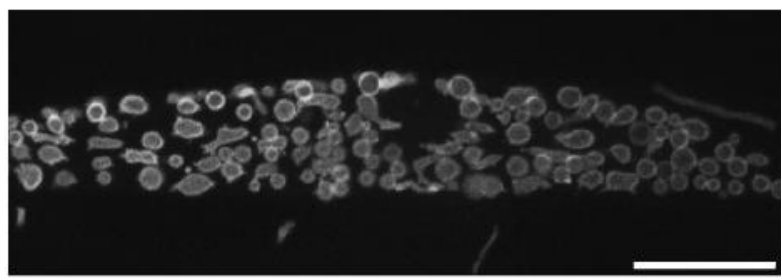

starved $52 \mathrm{~h}$ starting from L3

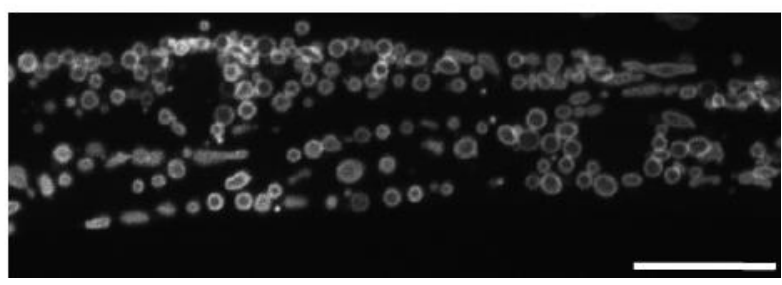

Fig. 4.9: Mitochondrial fragmentation in BWM cells upon starvation of wild type worms.

The impact of starvation on mitochondria in C. elegans muscle cells was assessed by fluorescence microscopy in wild type worms expressing the mitochondrial marker TOM70-CFP. While under non-starved conditions mitochondria form tubular structures (top panel), fragmentation of the mitochondria into vesicular structures can be observed upon starvation (as shown in the lower panels). Scale bars represent $10 \mu \mathrm{m}$. 
To examine whether similar effects on mitochondrial morphology might be observed in C. elegans, wild type worms expressing the mitochondrial marker TOM70-CFP in muscle cells were grown in the absence of food for $24 \mathrm{~h}$ starting from the L4 larval stage or for $52 \mathrm{~h}$ starting from L3.

As presented in Fig. 4.9, starvation induces mitochondrial fragmentation in C. elegans muscle cells. The resulting vesicular mitochondrial structure resembles the effects that were observed in aged wild type nematodes or young adult worms expressing wt aS.

\subsubsection{Fibril formation and mitochondrial fragmentation can also be seen in C. elegans neurons expressing aS}

As in PD neurons and not muscle cells are affected, we wanted to verify that the effects of $\alpha S$ on mitochondrial morphology are not specific to muscle cells but can also be seen in neurons. For this purpose I was expressing both the mitochondrial marker TOM70-CFP and wt $\alpha \mathrm{S}$ under the control of a pan-neuronal rab-3 promoter and was imaging neurons of the ventral nerve cord (VNC). Also in these neurons, wt aS is forming fibrillar aggregates as shown in Fig. 4.10A. These aggregates strongly resemble the aggregates that were seen in BWM cells. In muscle cells, the fibrillar aggregates usually measure around 5-15 $\mu \mathrm{m}$ with some longer aggregates of around $\sim 20 \mu \mathrm{m}$ observed in some cells. The aggregates in the smaller neuronal cell bodies typically have a length of around 2-10 $\mu \mathrm{m}$. While the fibrillar aggregates in muscle cells are mostly straight, the fibrils observed in neuronal cell bodies can either be straight or curved with some aggregates forming almost ring-like structures around the nucleus. Such bended fibrils were not seen in the BWM muscle cells and they might be caused by the small size of the neuronal cell bodies hindering straight fibrils from growing above a certain size.

Studying the mitochondrial morphology in VNC neurons revealed that mitochondria in the cell bodies of VNC neurons form long tubular (indicated by "T" above the arrowhead pointing towards the cell body) or ring-like structures (indicated by "R") around the nuclei in wild type worms (Fig. 4.10B). Also in the neurites that are projecting below the cell bodies in the VNC mitochondria appear as rather short but mostly tubular structures. In agreement to the changes in mitochondrial morphology in $C$. elegans muscle cells, expression of wt aS leads to fragmentation of mitochondria (indicated by "F") in both cell bodies and neurons of the VNC (Fig. 4.10C). Quantification of the mitochondrial morphologies was performed in two and three independent lines for worms without and with aS expression, respectively, and is depicted in Fig. 4.10D. The fact that expression of wt $\alpha \mathrm{S}$ causes mitochondrial fragmentation 
both in muscle cells and in neurons of $C$. elegans indicates that the effect is not cell-type specific.

A

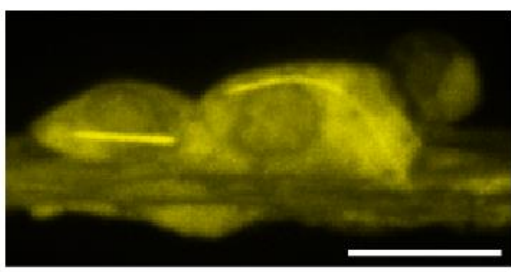

B

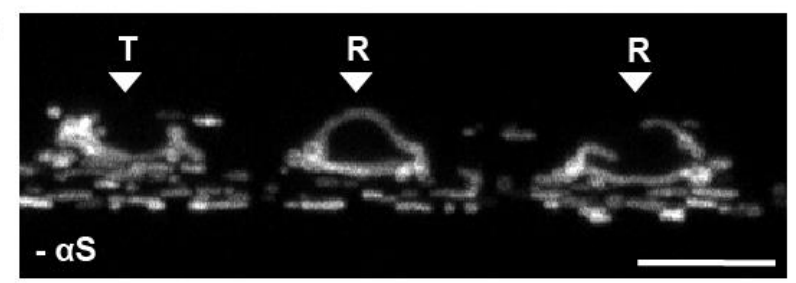

C

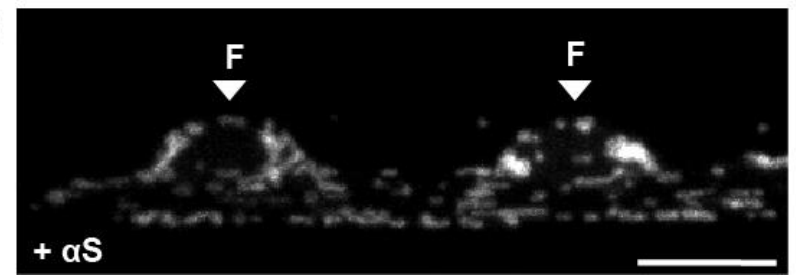

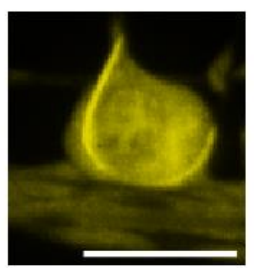

D

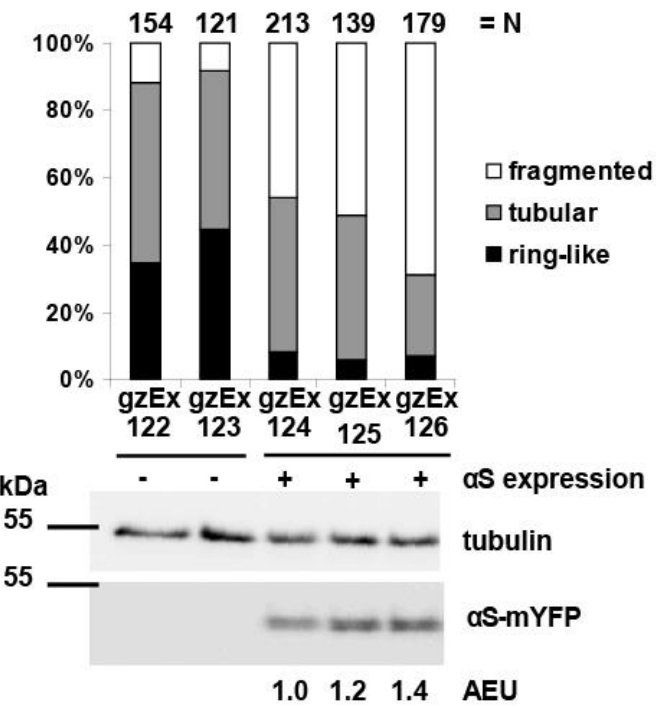

Fig. 4.10: Fibril formation and mitochondrial fragmentation in C. elegans neurons expressing wt aS.

(A) wt aS was tagged with mYFP at the C-terminus and expressed under the control of a pan-neuronal rab-3 promoter. Shown are neurons of the ventral nerve cord (VNC). Formation of fibrillar aggregates of aS-mYFP was observed. (B) Mitochondria in the cell bodies of VNC neurons of wild type worms not expressing aS form tubular or ring-like structures around the nucleus. (C) Expression of wt aS leads to mitochondrial fragmentation in VNC neurons. Neuronal cell bodies in $(B+C)$ are indicated by a white arrowhead. Classification of the mitochondrial morphology into the categories "ring-like" (R), "tubular" (T) or "fragmented" $(F)$ was done for each neuron and the category of the depicted neurons is indicated above the white arrowheads. Scale bars represent $5 \mu \mathrm{m}$. Pictures B-D were published in (Karpinar et al., 2009). 


\subsection{Discussion}

\subsubsection{Proline mutations in designed variants of aS prevent fibril formation in vitro and in vivo}

Post mortem analysis of brains from PD patients revealed that LBs are a characteristic feature of surviving $\mathrm{SN}$ neurons. As aggregated aS was reported to be the major component of LBs, it was assumed that fibrillar aggregates of aS cause neurotoxicity. Only in 2000, Lansbury and coworkers were postulating that oligomeric precursors of the fibrillar aggregates rather than the fibrils themselves might be the toxic species (Conway et al., 2000; Goldberg and Lansbury, 2000). This oligomer hypothesis was based on their observation that during the process of aS fibril formation, smaller oligomeric species are generated and that the rate of oligomer formation was increased in the A30P PD mutant (Conway et al., 1998; Conway et al., 2000). In contrast, only in the A53T mutant, an increased rate of fibril formation was observed, while several studies have demonstrated that the propensity to form fibrils is reduced in A30P when compared to wild type (Conway et al., 1998; Conway et al., 2000; Li et al., 2001). This indicates that at least in the case of A30P, disease pathogenesis is not directly correlated with increased formation of amyloid fibrils. The observation that aS oligomers can bind to liposomes and form pores in membranes thereby causing increased membrane permeability was providing further evidence for pathogenic effects of the pre-fibrillar species of aS (Volles et al., 2001). As it was not possible to directly assess in vivo, whether the occurrence of oligomeric aS correlates with toxicity, for many years there was still debate on which is the toxic species of aS. By using synthetic variants of aS which were designed to interfere with fibril formation and that instead promote oligomerization, we were able, for the first time, to clearly show in vivo that pre-fibrillar aS is exhibiting increased toxicity.

In vitro studies performed in the labs of Prof. Markus Zweckstetter and Prof. Christian Griesinger were confirming that the proline residues that were introduced in the designed variants A56P and TP (A30P/A56P/A76P) were indeed interfering with the formation of $\beta$-sheets and thus led to severely reduced rates of fibrillization (Fig. 4.2). Especially for TP no fibril formation as assessed by Thioflavin $\mathrm{T}$ fluorescence could be observed even after two weeks of incubation under conditions for which wt aS rapidly starts forming fibrils after a lag phase of approximately $20 \mathrm{~h}$ (Karpinar et al., 2009). Dynamic light scattering and NMR studies revealed that the soluble oligomers which precede the occurrence of amyloid fibrils during the course of aS aggregation are still present for A56P and TP (Karpinar et al., 2009). To exclude that these soluble oligomers that were observed for A56P and TP are "off-pathway" 
oligomers, which have different characteristics than the oligomers seen in wt aS and thus prevent the aggregation into fibrillar structures, incubations with high levels of protein and long incubation times were performed. Consistent with the oligomers of A56P and TP being "on-pathway", they could assemble to fibrils under these conditions (Karpinar et al., 2009). Furthermore, oligomers of TP $\alpha S$ were able to seed the aggregation of wt $\alpha S$, also supporting that they are "on-pathway" to fibrils (Karpinar et al., 2009). In agreement with the in vitro experiments, we were able to show that the designed variants are not forming fibrillar aggregates in vivo in contrast to wt aS (Fig. 4.3). Interestingly, we see fibrillar aggregates of wt aS-YFP in the muscles of transgenic worms used in this study while inclusions observed in previously reported strains expressing aS in $C$. elegans muscle cells were appearing as rather amorphous plaque-like structures (Hamamichi et al., 2008; van Ham et al., 2008). One explanation for this difference could be different aS constructs used, but all three strains are expressing aS fused to YFP or GFP at the C-terminus. Yet, it is conceivable that different linkers were used when creating the fusion proteins and that this might affect the aggregation properties. Furthermore, we used a myo-3 promoter instead of the unc-54 promoter that was used in the other studies. Both are strong promoters driving expression in the body wall and sex muscles of $C$. elegans, but the myo-3 promoter leads to a more stable expression throughout development while expression under the control of the unc-54-promoter results in very mosaic bursts of high expression levels particularly during early larval stages (S. Eimer, unpublished observation). To clarify this issue, it would be necessary to express our aS-YFP construct under the control of the unc-54 promoter and investigate the aggregates formed upon expression of this construct. Aggregate formation was studied in the vulva musculature and not in body wall muscles (BWMs) in our study but we find fibrillar aggregates also in the BWMs of our strains and even in neurons (Fig. 4.9). As aggregation of aS in vitro also yields in fibrillar aggregates, we are assuming that aS-YFP in our model is also forming amyloid fibrils. As the fibrils formed in vitro have a diameter of only around $10 \mathrm{~nm}$ (Choi et al., 2004; Conway et al., 1998; Giasson et al., 1999; Narhi et al., 1999) and conventional light microscopy is diffraction limited to approximately $200 \mathrm{~nm}$, we cannot test whether the dimension of the fibrils observed in $C$. elegans lies in the range of the in vitro fibrils. Thus, to test whether the fibrils seen in fluorescence microscopy are indeed made up of amyloid fibrils, it would be necessary to use EM or super-resolution imaging techniques like STED, STORM or PALM allowing a resolution of down to $20 \mathrm{~nm}$. Alternatively, staining the worms with Thioflavin $\mathrm{T}$ as described by Ved et al. (Ved et al., 2005), could be used to shed light on this aspect. Thioflavin $\mathrm{T}$ belongs to the group of benzothiazole dyes and exhibits fluorescence upon interaction with amyloid fibrils which is based on intercalation of Thioflavin $T$ within the amyloid fibrils (Hudson et al., 2009). Although the amyloid fibrils cannot directly be identified in fluorescence pictures of aS-YFP aggregates, the results of the in vitro analysis of TP aS and the 
fact that the introduction of proline residues in the TP variant abolishes the occurrence of these fibrils, clearly argues for the presence of amyloid fibrils. This was in agreement with work done in mammalian cell culture where fibril formation of the designed TP aS variant also prevented the formation of fibrillar aggregates (Karpinar et al., 2009). Thus, while wt aS is forming fibrillar aggregates in vitro and in vivo, the designed variant TP aS stops at a prefibrillar level, namely soluble oligomers. As it is not forming amyloid fibrils, it is a valuable tool for directly assessing the toxicity of oligomeric aS in vivo.

\subsubsection{TP $\alpha S$ exhibits increased neurotoxicity in $C$. elegans}

By comparing the toxicity of the designed pre-fibrillar $\alpha S$ variants with that of wt or familial PD mutant $\alpha S$ in $C$. elegans dopaminergic (DA) neurons, we could show that oligomeric species rather than fibrils are causing toxicity (Fig. 4.4) and thereby provide strong evidence for the oligomer hypothesis postulated by Lansbury and colleagues (Conway et al., 2000; Goldberg and Lansbury, 2000). Modest expression of wt $\alpha S$ does not lead to detectable neurite defects and also does not impair the worm's slowing in response to food, a behavior controlled by the dopaminergic system. This indicates that neither morphology nor function of the DA neurons was compromised. In contrast, expression of the PD mutants A53T and A30P induces defects in the neurites and these defects correlate with impaired function of the neurons as seen by reduced slowing of the worms when they encounter food. No obvious differences in toxicity between A53T and A30P could be observed in our model. Increased toxicity of A53T when compared to A30P had previously been reported in a study using transgenic mice (Lee et al., 2002) but we were not able to reproduce these results. However, a similar extent of neurodegeneration of $\mathrm{A} 53 \mathrm{~T}$ and $\mathrm{A30P}$ aS was also seen in another model of transgenic worms expressing aS in DA neurons (Kuwahara et al., 2006), indicating that the two PD variants are exhibiting similar levels of toxicity in C. elegans. Interestingly, the designed prefibrillar aS variants A56P and TP exhibited a significantly increased neurotoxicity compared to both wt and familial PD mutants. This was seen both on a morphological as well as a functional level. TP $\alpha \mathrm{S}$ was the most toxic variant with the effects on DA neurite morphology and on the food slowing response being much more pronounced than for A56P which still had some residual propensity to form amyloid fibrils. This strongly indicates that oligomeric precursors rather than $\alpha S$ fibrils are causing neuronal toxicity. The increased toxicity of PD mutants compared to wt $\mathrm{aS}$ is in line with this as increased oligomerization had been described for both mutants (Conway et al., 2000; Karpinar et al., 2009; Li et al., 2001). The fact that A53T and A30P cause neurodegeneration to a similar extent also argues for oligomers and not fibrils being the toxic species as A53T has a higher rate of fibril formation than A30P. Therefore, if fibrils were causing toxicity one would expect A53T to induce more severe de- 
fects. In agreement with these findings, experiments done in Drosophila and rat primary cortical neurons also demonstrated that TP aS exhibits the highest toxicity followed by A56P and the PD mutants all of which were causing increased defects when compared to wt $\alpha S$ (Karpinar et al., 2009).

Our results are in line with earlier experiments that had provided evidence for the oligomer hypothesis. The observation that chaperone expression was leading to decreased aS toxicity in Drosophila without changing the occurrence of fibrillar aggregates was indicating that the chaperones were acting on soluble aS oligomers and that those could be the toxic species (Auluck et al., 2002). Moreover, preventing phosphorylation of aS at Ser129 led to increased aggregate formation but was reducing toxicity in Drosophila, also arguing against fibrillar aggregates causing toxicity (Chen and Feany, 2005). A recently published study was using a similar approach of structure-based design than the one that we have presented to create $\alpha S$ mutants (E57K and E35K) which promote the formation of oligomeric aS without further aggregating to amyloid fibrils. Besides these fibrillar mutants, they were using a $\mathrm{C}$ - and $\mathrm{N}$ terminally truncated variant of aS ( $\alpha S 30-110$ ) that is characterized by an increased rate of fibrillization as well as the three known PD mutants A30P, A53T and E46K and wt aS and were examining the toxicity of the different variants in rats. Thus, using an in vivo mammalian system they were able to confirm our results by showing increased toxicity of oligomeric aS compared to fibril forming species (Winner et al., 2011). They also showed that that the toxic oligomeric species bind to membranes forming pore-like structures suggesting that thereby induced changes in permeability and integrity of cellular membranes are causing toxicity.

The finding that oligomeric forms rather than the amyloid fibrils are causing neurotoxicity seems not to be unique to PD but it was also proposed for other neurodegenerative diseases like Alzheimer's disease and Huntington's disease. All of these diseases belong to the group of amyloid diseases which are characterized by the presence of fibrillar protein aggregates. While in PD $\alpha S$ is the aggregating protein, fibrillar aggregates of $\beta$-amyloid and huntingtin are found in Alzheimer's disease and Huntington's disease, respectively. Besides the insoluble fibrils, soluble oligomeric species have been described for all three diseases and it was postulated that these pre-fibrillar aggregates are responsible for toxicity [reviewed in (Glabe, 2006)].

The relevance of soluble oligomers for PD pathogenesis was further emphasized by the finding that levels of oligomeric aS are elevated in patients suffering from PD (Sharon et al., 2003). Moreover, it was reported that auto-oxidation of dopamine leads to a stabilization of oligomeric aS (Conway et al., 2001; Norris et al., 2005), an observation that might explain the 
particular vulnerability of DA neurons of the substantia nigra. The finding that pre-fibrillar species of $\alpha S$ are responsible for aS-induced neurotoxicity also has implications for PD treatment. Thus, preventing the oligomerization of $\alpha S$ seems to be a promising target for PD therapy. When searching for possible substances that could prevent aggregation of $\alpha \mathrm{S}$, one therefore has to be very careful that not only the more easily detectable insoluble fibrils disappear but that also the small soluble precursors are not formed in the presence of the drug. As oligomers and not fibrils seem to be the toxic species of $\alpha S$, it might even be neuroprotective to trigger aggregation of small oligomers to larger fibrillar aggregates though it is not sure whether huge inclusion bodies in the cell might not lead to compromised function to some extent as well. Besides that, changing the equilibrium from oligomeric to fibrillar aggregates might also induce an increased formation of new oligomers from monomeric aS so that the toxic load might stay unchanged. Therefore, it seems more promising to screen for substances that block the conversion of monomeric aS to oligomers or that trigger formation of non-toxic oligomeric variants. As it has been postulated that toxicity of the oligomers might be induced by interaction with cellular membranes, another possibility to interfere with toxicity would be finding molecules that are able to bind to the oligomers and hinder them from binding to phospholipids. Transgenic worms expressing oligomer forming TP $\alpha S$ as described in this study might be a valuable tool to screen for therapeutic drugs that prevent toxicity induced by oligomeric aS species.

\subsubsection{Expression of $\alpha \mathrm{S}$ leads to drastic changes of mitochondrial morphology in C. elegans muscle cells and neurons}

After having identified soluble oligomers as toxic species of $\alpha \mathrm{S}$, we were aiming in understanding the mechanism how aS mediates toxicity on the cellular level. In the past years, increasing evidence for involvement of mitochondria in the pathogenesis of PD [reviewed in (Bueler, 2009; Burbulla et al., 2010)] and other neurodegenerative diseases [reviewed in (Chen and Chan, 2009; Knott et al., 2008)] was found. Furthermore, a link between aS and this cellular compartment was reported. Several studies have found that aS localizes to mitochondrial membranes (Chinta et al., 2010; Devi et al., 2008; Li et al., 2007; Nakamura et al., 2008; Parihar et al., 2008) leading to increased oxidative stress (Hsu et al., 2000; Parihar et al., 2008; Poon et al., 2005; Smith et al., 2005), reduced activity of complex I of the respiratory chain (Chinta et al., 2010; Devi et al., 2008), cytochrome c release and induction of apoptosis (Parihar et al., 2008; Smith et al., 2005) as well as changes in mitochondrial morphology (Hsu et al., 2000). To examine whether aS expression affects mitochondria in our C. elegans model, we were using confocal microscopy and HPF-EM to study the morphology 
of mitochondria in worms expressing different concentrations of wt aS as well as the above described pre-fibrillar variant TP and the familial PD mutants A30P and A53T.

Expression of wt $\alpha \mathrm{S}$ in $C$. elegans muscle cells strongly influences mitochondrial morphology (Fig. 4.5). Mitochondria in the BWMs of wild type worms form tubular structures in a regular arrangement in parallel to the myo-filaments. Upon expression of wt $\alpha \mathrm{S}$, mitochondria become either fragmented or form networks of long, thin and highly interconnected mitochondria. Mitochondrial fragmentation leads to vesicular shape of the mitochondria of varying diameter. While in some cells all the mitochondria appear to be fragmented, in other cells only long, thin and interconnected mitochondria can be observed and there were also cells in which both fragmented and elongated structures are present.

HPF-EM analysis confirmed the effects of $\alpha S$ on mitochondrial morphology that were seen with fluorescence microscopy on an ultra-structural level (Fig. 4.6). Mitochondria in BWM cells expressing wt aS have a clearly reduced diameter compared to those in in wild type worms. In contrast, no obvious defects in cristae structure were observed upon expression of wt aS. As only single sections were analyzed by EM it is difficult to assess differences between fragmented and long and thin mitochondria as the latter ones would only differ from the fragmented ones if they were cut longitudinally. Thus, the complementary analysis by EM and confocal microscopy is most powerful in dissecting different aspects of mitochondrial morphology changes. While confocal microscopy gives a good overview of the overall morphology and distribution of mitochondria in the entire 3D volume of the cell and thus enables us to distinguish between elongated, network-like and fragmented, vesicular morphologies, HFP-EM provides us with information on the ultra-structural changes like cristae structure and mitochondrial diameter. Another advantage of EM analysis is that it facilitates the investigation of various cellular compartments. While for confocal microscopy markers for every compartment would need to be used and thus various transgenic lines would need to be created, all compartments are readily visible in EM pictures. While changes in mitochondrial morphology were easily seen in the EM pictures of muscles expressing wt aS, no obvious structural changes in other cellular compartments were observed. aS in our model seems to rather specifically affect the structure of mitochondria without changing the morphology of other organelles. This is in contrast to previous studies which reported Golgi fragmentation upon expression of aS (Gosavi et al., 2002; Lee et al., 2006). It is possible that the effects on Golgi morphology differ between different cell types and organisms. Furthermore, we did not do a detailed analysis of all compartments but were rather looking for gross morphological changes. Thus, more subtle changes on the Golgi could have been missed. In a recent study, Nakamura et al. also showed that aS expression causes mitochondrial fragmentation 
without affecting the morphology of other organelles which is in line with our observation (Nakamura et al., 2011). As described above, expression of wt $\alpha \mathrm{S}$ led to mitochondrial fragmentation in some cells, whereas networks of long and thin mitochondria where found in others. It is not clear what determines whether cells become fragmented or rather elongated in the presence of $\alpha S$ and whether one of the two effects precedes the other. We sought to investigate the latter aspect by expressing different concentrations of aS. We were hypothesizing that, if one of the effects is occurring earlier, this one might predominantly be seen with low expression levels of $\alpha \mathrm{S}$. Though, even with low concentrations, both morphologies could be observed and no obvious change in the relative occurrence of the two morphologies compared to higher aS concentrations could be detected (Fig. 4.5). Although the different expression levels did not affect the ratio between elongated thin and fragmented mitochondria, we were still able to determine that the effect of $\alpha \mathrm{S}$ on mitochondrial morphology is concentration-dependent. In worms expressing aS at low concentrations, mitochondria in $60-80 \%$ of the cells are still showing wt-like morphology. In contrast, wt-like mitochondria are present in only $10-20 \%$ of the worms expressing aS at high levels. Furthermore, we found a saturation effect as increasing aS above a certain level did not cause an aggravation of the phenotypes anymore. As the analysis of different concentrations of aS did not answer our question whether fragmentation and elongation are consecutive events, we thought of other ways to investigate this aspect and decided to investigate mitochondrial morphology in aged worms expressing $\alpha \mathrm{S}$. At $20^{\circ} \mathrm{C}$, wild type $C$. elegans have a mean lifespan of about 2-3 weeks and take 3 days to reach adulthood. We were expecting that, if one of the morphological changes was preceding the other, the late-stage morphology should be more abundant in 7-day-old worms. Investigation of aged worms expressing aS revealed that both fragmented and elongated mitochondria are still present at late stages (Fig. 4.8). The relative occurrence of fragmented mitochondria was slightly increased compared to young worms expressing aS but as mitochondrial fragmentation also occurs in aged worms without expression of $\alpha \mathrm{S}$, this effect might rather be due to the aging process itself and independent of $\alpha \mathrm{S}$ expression. These results suggest that both events - mitochondrial fragmentation and the formation of networks of long and thin mitochondria - are occurring in parallel to each other and are not necessarily consecutive events. It remains to be determined which factors are determining which morphological changes of the mitochondria are induced by aS and it will also be interesting to examine whether one of the two conditions is more harmful to the cell. It is possible that different species of aS (monomer vs. different oligomeric forms or fibrils) are affecting mitochondrial morphology in different ways and examination of the effects of different variants of aS might therefore help to get a better understanding of this aspect. 
Therefore, we examined the mitochondrial morphology in muscle cells expressing different $\alpha S$ mutants. Besides wt $\alpha S$ and two PD mutants (A30P and A53T) the designed pre-fibrillar TP variant was included in this analysis. The effect of A53T aS is very similar to the one seen for wt aS with both fragmentation and elongation to thin tubular structures being observed and only $20 \%$ of cells exhibiting wt-like mitochondrial morphology. In contrast, the second PD mutation, A30P, seems to be less deleterious with changes of mitochondrial morphology occurring in only $40-70 \%$ of the cells even though the expression levels, as analyzed by Western blot, were markedly higher than for wt or A53T (Fig. 4.7A). The ratio of mitochondrial fragmentation and elongation in the affected cells is similar to the ones upon expression of wt or A53T aS.

Interestingly, expression of the mainly oligomeric TP aS predominantly leads to long, thin and interconnected mitochondria and less mitochondrial fragmentation is observed (Fig. 4.7A). The total amount of cells in which mitochondrial morphology is affected by TP aS is comparable to the one of wt aS. Analysis of mitochondria of worms expressing TP aS was also done by HPF-EM (Fig. 4.7B). A drastic decrease in mitochondrial diameter was observed for TP as compared to wild type worms and in longitudinal sections even elongated thin mitochondria were found. Again, as for wt $\alpha S$, no obvious changes in the cristae structure were observed upon expression of TP aS. It has to be noted that expression levels of TP aS were much higher in all three transgenic lines than in the lines expressing wt $\alpha \mathrm{S}$ so that it cannot be completely excluded that increased concentration of TP aS is the reason for different extents of mitochondrial changes. We tried to generate lines of comparable expression levels by injecting the same plasmid concentrations for all constructs but the number of copies of a gene in extrachromosomal arrays in C. elegans is determined stochastically. However, when examining the effect of different concentrations of wt aS, we observed a saturation of the effects at a certain concentration, arguing against increased concentrations of TP $\alpha S$ being able to explain the differences in morphology. Furthermore, expression levels of the PD mutant A30P were in the range of those of TP aS and still did not induce such strong effects. In the meantime, transgenic lines expressing TP aS at lower concentrations were generated with help of David Vasmer, a lab rotation student. Even with these lower concentrations, the predominant morphology of the mitochondria in muscle cells seems to be long, thin and interconnected tubules. Therefore, we propose a model in which differences in the effects between wt and TP $\alpha$ S are originating from different properties and/or amounts of the oligomeric species that are formed by the two proteins. As discussed in the previous sections of this chapter, TP aS differs from the wt protein in its aggregation properties. While wt $\alpha S$ forms amyloid fibrils, aggregation of TP aS stops at the stage of soluble oligomers (Fig. 4.2 and 4.3). We also showed that TP aS leads to increased neurotoxicity in C. elegans DA neu- 
rons (Fig. 4.4). Hence, one possibility would be that oligomeric species like the ones formed by TP aS bind to mitochondria in a way in which they can induce elongation to very thin tubules and as these oligomeric species are present in lower amounts for wt $\alpha \mathrm{S}$, mitochondrial elongation is observed to a lower extent. Another scenario would be that pre-fibrillar TP aS induces toxicity in the cells through a mechanism that is independent of its direct effects on mitochondria and that these "pre-damaged" cells react to binding of $\alpha S$ to the mitochondria with different changes in mitochondrial morphology.

Another important aspect that might explain differences in the effects of the various mutants on mitochondrial morphology is their propensity to bind membranes. It has been shown that the ability of A30P to bind to membranes is reduced when compared to wt aS (Karpinar et al., 2009; Perrin et al., 2000). Therefore, if aS affects mitochondrial morphology by a mechanism that involves binding of the protein to the mitochondrial membrane, this should lead to milder effects than in the case of wt $\alpha S$ and A53T which bind to membranes more efficiently. Indeed, we see similar changes in mitochondrial morphology for wt and A53T, while mitochondria in cells expressing even higher levels of A30P show only minor defects. The membrane binding properties of TP $\alpha$ S have been determined in vitro in the lab of Prof. Markus Zweckstetter and were found to be similar to those of A30P aS or even a bit lower (Karpinar et al., 2009). This is surprising as we see drastic changes in mitochondrial morphology towards networks of long and thin mitochondria for TP aS. This could mean that either the binding of TP aS in vivo differs from the one in vitro - also the composition of mitochondrial membranes could differ significantly from the membranes used for the in vitro studies - or mitochondrial elongation is caused by indirect effects of TP aS on mitochondrial morphology. Further investigations of the ability of TP aS to bind to mitochondria in vivo, for example by using immuno-EM, are needed in order to be able to decipher the mechanism by which TP aS leads to mitochondrial elongation. Furthermore, it would be interesting to study the effect of $\alpha S$ variants that lack the ability to bind membranes and see whether they can still influence mitochondrial morphology. If this is the case, this would argue for more indirect effects of $\alpha S$ on mitochondrial dynamics. If such mutants would, in contrast, not affect mitochondrial morphology anymore, this would clearly indicate that aS influences mitochondrial morphology through direct binding to the mitochondrial membrane. Moreover, investigating the effects of E46K aS, another PD mutant, could be helpful in deciphering the role of membrane binding for mitochondrial morphology as this mutant was shown to exhibit increased phospholipid binding properties (Choi et al., 2004). We are currently creating transgenic lines expressing E46K aS and it will be very interesting whether increased mitochondrial fragmentation will be observed in these strains. 
Notably, expression levels in our transgenic worms were much higher for TP and A30P than for wt and A53T aS despite the fact that the same plasmid concentrations were used when generating the transgenic worms. Although the expression levels in different transgenic lines can vary with the same injection mix, it is unusual that the expression of all three independent lines for TP and the two lines of A30P aS are 3-7-fold higher than all three lines of wt $\alpha S$ and the line expressing A53T. Thus, it is possible that increased stability of TP and A30P aS contributes to the increased protein levels. Effects of aS on cellular degradation systems including both UPS and autophagy, have been described (Cuervo et al., 2004; MartinezVicente et al., 2008; Snyder et al., 2003; Winslow et al., 2010) and it is possible that the different aS species affect these systems to a different extent. Further investigations will be needed to figure out whether this is the reason for divergent protein levels of TP and wt aS in our transgenic $C$. elegans strains. To do so, one would need to use inhibitors for proteasomal and autophagic degradation and investigate whether under these conditions wt aS levels are increasing to a similar level than those seen for TP aS.

Our collaborators in the lab of Prof. Christian Haass (LMU Munich) were investigating the effect of aS overexpression on mitochondrial morphology in SH-SY5Y cells. In line with our observations, they see mitochondrial fragmentation upon expression of wt and PD mutant $\alpha S$ (Kamp et al., 2010). In their system, no differences in the effects of wt and PD mutant aS were observed (Kamp et al., 2010). They were also expressing $\beta$-synuclein ( $\beta S$ ), a homolog of $\alpha S$ that lacks a stretch of hydrophobic amino acids in the central region which is present in $\alpha S$. Similar to $\alpha S, \beta S$ also binds to membranes (Varkey et al., 2010) but it differs from $\alpha S$ in the propensity to aggregate. Due to lack of the central hydrophobic region, $\beta S$ is not prone to aggregation (Biere et al., 2000; Giasson et al., 2001; Serpell et al., 2000; Uversky et al., 2002). Strikingly, mitochondrial fragmentation was also seen upon expression of $\beta S$ (Kamp et al., 2010), indicating that binding of monomeric species of synucleins inhibits membrane fusion. It is possible that oligomeric aS can also bind to mitochondrial membranes but affects morphology in a different way, rather producing long tubular structures as seen in the oligomer-promoting variant TP $\alpha \mathrm{S}$. Work in the lab of Christian Haass also revealed that binding of $\alpha S$ to mitochondrial membranes inhibits fusion rather than promoting fission as seen in cell fusion assays where differently labeled mitochondria did not mix when aS was expressed (Kamp et al., 2010). They could also show that cellular ATP levels and mitochondrial membrane potential are not affected by aS overexpression, indicating that it is unlikely that mitochondrial defects are responsible for the changes in mitochondrial dynamics (Kamp et al., 2010). Moreover, they found that the effect of aS on mitochondrial morphology were independent of the mitochondrial dynamics genes Mfn1, Mfn2, OPA1 and Drp1 and that the levels of these proteins as well as OPA1 processing were not changed by aS (Kamp et al., 
2010). Therefore, it seems likely that binding of aS directly inhibits fusion of the membranes rather than disturbing the function of the proteins regulating mitochondrial dynamics.

Recently, another study was published in which the effects of different aS constructs on mitochondrial morphology were assessed in mammalian cells, both in vitro and in vivo. In agreement with our observations they also described mitochondrial fragmentation upon expression of wt aS and did not see strong effects on the morphology of other organelles (Nakamura et al., 2011). Interestingly, similar to what we had reported for $C$. elegans BWMs, they also saw weaker effects of A30P aS on mitochondrial dynamics, indicating that reduced membrane binding as described for A30P (Choi et al., 2004; Karpinar et al., 2009) prevents aS from influencing mitochondrial morphology. In line with membrane binding playing an essential role for the induction of mitochondrial fragmentation, mutated variants of $\alpha S$ that fail to associate to membranes do not induce fragmentation (Nakamura et al., 2011). Nakamura et al. also report that $\beta S$ can also induce mitochondrial fragmentation but that the effects are not as prominent as for $\alpha S$ and only negligible effects were found upon expression of $y$ synuclein (Nakamura et al., 2011). Our collaborators in the lab of Prof. Christian Haass, in contrast, had observed the same degree of fragmentation for both $\alpha$ - and $\beta S$ (Kamp et al., 2010). Further investigation of this aspect will be needed as it might give insight into which species of synuclein (monomers or oligomers) is causing the effects. As $\beta S$ has markedly reduced aggregation propensity, fragmentation to the same levels as for aS would argue for monomeric variants as inducers of fragmentation while reduced levels in the case of $\beta S$ as reported by Nakamura et al. would argue in favor of oligomeric species being responsible for mitochondrial fragmentation. In agreement with the results of the cell culture studies performed in the lab of Prof. Christian Haass, no changes in mitochondrial membrane potential, superoxide levels or oxygen consumption were seen in cells expressing aS (Nakamura et al., 2011) arguing for mitochondrial changes being induced specifically by the interaction of $\alpha S$ with mitochondria rather than through compromised mitochondrial function. While EM analysis of the mitochondria in our $C$. elegans model as well as in the SH-SY5Y cells expressing aS did not reveal any abnormalities in mitochondrial cristae structure (Kamp et al., 2010), Nakamura at al. observe disordered cristae in both cell culture and transgenic mice. In summary, mitochondrial fragmentation has been observed by us in $C$. elegans muscle cells, mammalian cell culture (Kamp et al., 2010) and by Nakamura et al., in mammalian cell culture and transgenic mice (Nakamura et al., 2011). Direct binding of aS to mitochondrial membranes seems to be responsible for induction of fragmentation but further studies, investigating in more detail how different monomeric and oligomeric forms influence mitochondrial dynamics, are needed. This is particularly important as the oligomer promoting variant TP aS 
seems to affect mitochondria in a different way and is rather inducing mitochondrial elongation and network formation than mitochondrial fragmentation.

When examining mitochondrial morphology of aged worms in the post-reproductive phase, we observed fragmented, vesicular mitochondria in about $40 \%$ of the cells (Fig. 4.8). This indicates that mitochondrial fragmentation is part of the physiological mitochondrial aging process. As the same amount of fragmented mitochondria is observed in young adult worms expressing wt $\alpha S$, it is tempting to speculate that $\alpha S$ accelerates the physiological aging process. In aS expressing worms, an increase in the portion of fragmented mitochondria with aging can also be found. This effect, though, is not as pronounced as in wild type worms. While mitochondrial fragmentation seems to be a common feature of mitochondrial aging in wild type worms, in the aged wild type animals, only very few cells show long and thin mitochondria like the ones observed upon expression of wt or even more pronounced upon expression of TP aS. As we did not follow the complete aging process - we were just examining 7-day old compared to 3-day old worms - and given that the mean life span of $C$. elegans is 12-18 days, it is conceivable that such alterations emerge only in later stages of the aging process. On the other hand it is also possible that these alterations are not part of the physiological aging process but rather unique effects of expression of $\alpha \mathrm{S}$.

Mitochondrial fragmentation similar to that seen upon expression of wt $\alpha \mathrm{S}$ has been reported to be an early sign of apoptosis [reviewed in (Youle and Karbowski, 2005)] and it will therefore be interesting to follow these cells over time and determine whether they indeed are undergoing cell death. Furthermore, mitochondrial fragmentation could facilitate mitochondrial clearance via mitophagy and massive loss of mitochondria might be detrimental to the cell (Gomes et al., 2011). Moreover, inhibition of fusion has been shown to induce deficiencies in the respiratory chain, probably because lack of fusion prevents complementation between mitochondria [reviewed in (Otera and Mihara, 2011)]. But also the elongated and thin mitochondria that were found in TP aS expressing cells could compromise mitochondrial function as fragmentation of mitochondria is a prerequisite for mitophagic clearance for damaged mitochondrial components (Twig et al., 2008b) and accumulation of damaged material might compromise mitochondrial function. Furthermore, mitochondrial elongation induced by expression of TP aS could be particularly problematic in neurons as they rely on transport of mitochondria along the neurites which is hindered by very large mitochondrial networks [reviewed in (Westermann, 2010b)]. In contrast, it was also reported that mitochondrial elongation can serve as a protective mechanism against apoptosis [reviewed in (Youle and Karbowski, 2005)] and excess mitophagy as it was reported for conditions of nutrient deprivation (Gomes et al., 2011). Therefore, an interesting question to be addressed in future 
studies is how the changes in mitochondrial morphology affect the cellular fitness. In this respect, it will be particularly interesting to compare mitochondrial function and cellular fitness between cells with fragmented mitochondria and those in which mitochondria are forming elongated networks. Yet, it will be challenging to investigate this aspect as the methods commonly used for assessing mitochondrial function rely on measurements of whole animals (oxygen consumption, ATP levels, ROS production) or are based on investigation of isolated mitochondria that have been extracted from whole worms. We are currently generating worms expressing different $\alpha \mathrm{S}$ from a ubiquitous promoter so that we could at least investigate the overall effects of different $\alpha S$ variants on mitochondrial morphology. However, to clearly determine the correlation of morphology and function, techniques will have to be established that allow assessment of mitochondrial function in individual cells as we have observed that the effect of aS expression on mitochondrial morphology varies between cell types and even between cells of the same tissue.

It had been reported that the nutrient status of cells has an important impact on mitochondrial dynamics with starvation causing the occurrence of elongated mitochondria (Gomes et al., 2011). We were thus investigating whether this is also the case for mitochondria in C. elegans BWM cells. As we saw mitochondrial elongation upon expression of wt $\alpha \mathrm{S}$ and even more pronounced when TP $\alpha$ S was expressed, we were hypothesizing that the underlying mechanism could be similar to what is happening under conditions of nutrient deprivation. Gomes et al., had reported that depletion of nutrients leads to cAMP-induced activation of PKA which phosphorylates the mitochondrial fission protein DRP-1. As a consequence, DRP-1 is residing in the cytosol and cannot promote mitochondrial fission. It was furthermore shown that this mechanism serves to protect mitochondria from being degraded via autophagy which is induced upon starvation. It was reported that autophagy is also induced upon expression of aS (Stefanis et al., 2001; Xilouri et al., 2009) and it was even shown that massive mitochondrial degradation via aS-induced autophagy might cause cell death and that inhibiting the targeting of mitochondria to autophagosomes is protective (Choubey et al., 2011). Thus, mitochondrial elongation in cells expressing aS could also serve as a mechanism to protect the cells against massive degradation of mitochondria. In contrast to the observations in mammalian cell culture, we did not see mitochondrial elongation in muscle cells of wild type worms starved for 24-52 h but we rather found fragmented mitochondria in muscle cells of these animals (Fig. 4.9). It remains to be determined whether mitochondrial elongation upon starvation is only observed in some cell types or whether we did not find the right starvation conditions and time windows. Concerning the starvation conditions, the effect might differ depending on which nutrients are depleted, e.g. whether worms are depleted from amino acids or from glucose. Nevertheless, even if mitochondrial elongation was not 
found upon nutrient deprivation of nematodes, the mechanism inducing elongation upon expression of wt $\alpha \mathrm{S}$ could still be analogous to the one that had been described in cell culture by Gomes et al. and could be protective by preventing excess mitochondrial degradation and also ensuring sufficient supply with ATP.

After having studied mitochondrial morphology in $C$. elegans BWMs we also examined the effects of $\alpha S$ expression on mitochondrial dynamics in neuronal cells and found that severe mitochondrial fragmentation is induced by expression of wt $\alpha \mathrm{S}$ (Fig. 4.10). While ring-like and tubular morphology of the mitochondria was seen in wild type worms, punctate structures were found in the cells expressing aS. Also in the neurites, mitochondrial length was drastically reduced upon expression of $\alpha S$. This indicates that $\alpha S$ leads to mitochondrial fragmentation in a tissue-independent manner in $C$. elegans. On the contrary, formation of networks of long and thin mitochondrial tubules as seen in BWMs was not observed in the neurons. It is possible that this morphology would have detrimental effects to nerve cells as they are very dependent on transport of mitochondria along their processes and it is easier to transport smaller structures than long and extended networks. This could also be a possible explanation of why in the SH-SY5Y cell line only mitochondrial fragmentation was observed when expressing aS. As SH-SY5Y cells are a neuroblastoma cell line, mitochondrial dynamics might resemble the one of neuronal cells rather than that of other cell types and thus mitochondrial elongation mechanisms might not be very pronounced. We did not study mitochondrial morphology in neurons of worms expressing TP aS but it would be very interesting to see whether this variant also induces fragmentation when expressed in neurons as in the case of wt $\alpha S$ or whether then also mitochondrial elongation would be observed. If mitochondrial elongation was found in neurons upon expression TP aS, this might explain the increased neurotoxicity of this variant.

To conclude, we have shown that expression of aS affects mitochondrial morphology in C. elegans muscle cells and neurons. While in neurons aS predominantly induces mitochondrial fragmentation, in muscle cells, besides fragmentation, formation of long and thin tubules can be seen in some cells. This mitochondrial elongation is furthermore the predominant morphology found when the pre-fibrillar TP $\alpha \mathrm{S}$ is expressed suggesting that oligomeric forms of $\alpha S$ might affect mitochondrial morphology in a different way than monomeric aS. Given the observation that in cell culture, $\beta S$ leads to mitochondrial fragmentation similar to $\alpha S$, it seems likely that binding of monomeric forms of aS to mitochondria is causing fragmentation because $\beta S$ does not form aggregates. Studies in cell culture point towards aS inhibiting membrane fusion rather than promoting fission (Kamp et al., 2010). How mitochondrial elongation is triggered by expression of TP $\alpha \mathrm{S}$ and also to some extent by wt $\alpha \mathrm{S}$ will be an inter- 
esting target for future investigations. The observation that binding of $\alpha \mathrm{S}$ to mitochondria induces changes in mitochondrial morphology might be of great relevance for the pathogenesis of PD as increased levels of mitochondria-associated aS have been found in the SN of PD patients (Devi et al., 2008). Re-establishing the balance between mitochondrial fission and fusion might be a promising target for the establishment of new treatments for PD. 


\section{Chapter II: Cellular function and toxicity of catp-6 (PARK9) in C. elegans}

\subsection{Introduction}

In humans, mutations in ATP13A2/PARK9 have been identified as the cause of Kufor-Rakeb syndrome (KRS) (Ramirez et al., 2006), a rare form of Parkinsonism that is characterized by pyramidal degeneration, dementia and supranuclear gaze palsy in addition to typical parkinsonian motor symptoms (Behrens et al., 2010; Najim al-Din et al., 1994; Ramirez et al., 2006; Williams et al., 2005). C. elegans catp-6 has been shown to be the ortholog of ATP13A2 (PARK9) (Gitler et al., 2009). While the initially described mutations in ATP13A2 in KRS patients were homozygous or compound heterozygous mutations resulting in truncated variants of the protein due to frame shifts or splicing variants (Ramirez et al., 2006), more recently also heterozygous frame shift mutations and missense mutations, either homo- or heterozygous, were found in patients suffering from early onset PD (Di Fonzo et al., 2007; Djarmati et al., 2009; Fong et al., 2011; Lin et al., 2008) and some of them have been proposed as risk factors for developing sporadic PD (Chen et al., 2011; Di Fonzo et al., 2007). Phenotypes in patients with missense mutations seem to be milder than for frame shift mutations and splicing variants and mostly lead to development of typical parkinsonian signs without additional symptoms as seen in KRS patients (Lees and Singleton, 2007).

ATP13A2 belongs to the $\mathrm{P}_{5}$ subfamily of P-type ATPases (Kuhlbrandt, 2004; Moller et al., 2008; Schultheis et al., 2004) for which the substrate specificity remains unclear. It was proposed that ATP13A2 is involved in transporting divalent cations like $\mathrm{Cd}^{2+}$ and $\mathrm{Mn}^{2+}$ as increased sensitivity to these cations was observed in yeast in deletion mutants of Ypk9, the yeast ortholog of ATP13A2 (Gitler et al., 2009; Schmidt et al., 2009). Studies in mice revealed that ATP13A2 is ubiquitously expressed with strong expression in the brain, predominantly in neurons and particularly high expression levels were found in DA neurons of the SN (Ramonet et al., 2012; Schultheis et al., 2004). Interestingly, elevated levels of ATP13A2 were found in the SN of PD patients (Ramirez et al., 2006; Ramonet et al., 2012), indicating that this gene not only plays a role in familial PD but changes in the expression level might also provide a link to the more common sporadic forms of the disease. A recent study investigating the temporal expression pattern of ATP13A2 expression in mice found that the highest expression levels were reached at the time of neurogenesis and it overlapped with the time window in which the midbrain including basal ganglia and striatum are forming. As these 
are the structures that are mainly affected in PD this further substantiates the link between ATP13A2 and PD.

Lysosomal localization has been reported for wt ATP13A2 (Park et al., 2011; Ramirez et al., 2006; Ramonet et al., 2012; Schroder et al., 2007; Tan et al., 2011; Ugolino et al., 2011) and it was shown that truncation of the protein by mutations in KRS patients leads to retention in the ER (Ramirez et al., 2006; Tan et al., 2011; Ugolino et al., 2011). This accumulation of unstable mutant ATP13A2 induces the unfolded protein response in the ER (Park et al., 2011) and renders cells more vulnerable to ER stress induced by tunicamycin (Park et al., 2011; Ugolino et al., 2011). Moreover, it was reported that the half-life of mutant ATP13A2 is reduced when compared to the wild type protein (Ugolino et al., 2011) and degradation studies have revealed that it is degraded by the proteasome (Park et al., 2011; Ramirez et al., 2006; Ugolino et al., 2011) and that mutant ATP13A2 is cleared via the ERAD (ER associated degradation) pathway (Park et al., 2011; Ugolino et al., 2011).

While many studies have been published on linkage analysis between ATP13A2 and PD, not much is known about the cellular function of this protein and the pathogenic mechanisms leading to disease when mutated. Only recently, a number of studies have begun to shed light on the cellular role of ATP13A2 and on how mutant ATP13A2 causes toxicity. Given the genetic tractability of $C$. elegans and the finding that many PD-associated genes are conserved between humans and $C$. elegans, we decided to use this nematode as a model system to gain insight into the cellular function of ATP13A2. C. elegans has three orthologs of ATP13A2: CATP-5, CATP-6 and CATP-7. Heinick et al. have reported that CATP-5 is localized to the plasma membrane in intestinal cells and the excretory cell (Heinick et al., 2010) and as both expression pattern and subcellular localization of CATP-5 differ from ATP13A2, it seems rather unlikely that they are isofunctional homologs. As a functional link between CATP-6 and aS had been previously reported with co-expression of CATP- 6 ameliorating aS-induced toxicity in yeast, C. elegans and rat primary neuronal cultures (Gitler et al., 2009), we decided to focus on investigating the cellular function of this protein. 


\subsection{Results}

\subsubsection{Gene structure of catp-6}

The C. elegans catp- 6 gene spans $7.2 \mathrm{~kb}$ of genomic DNA and consists of 21 exons. It is located on chromosome IV and two deletion mutants for this gene are available. The gene structure as well as the position of the deletions are depicted in Fig. 5.1. The gene is the ortholog of human ATP13A2(PARK9) and the two proteins have the same domain structure. An alignment of the two proteins is provided in the appendix (section 8.1).

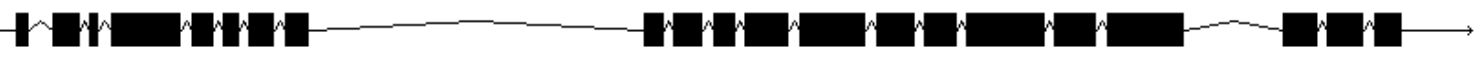 \\ $\overline{\operatorname{tm} 3190}$ \\ ok3473 \\ $1 \mathrm{~kb}$}

Fig. 5.1: Gene structure of catp-6 and position of deletions.

Exons are depicted as black boxes and introns are represented as black lines. The catp- 6 gene consists of 21 exons (black boxes) and spans $7.2 \mathrm{~kb}$ of genomic DNA. The position of $t m 3190$ and $0 \mathrm{k} 3473$ deletions is indicated as red lines.

\subsubsection{Deletion of catp-6 leads to egg laying defects, causing a reduction in brood size}

The first aspect of the mutant analysis was a phenotypic characterization which revealed that both deletion mutants of catp-6 are egg laying defective (Egl). This can be seen in DIC pictures of the worms by an accumulation of eggs in the uterus of the mutants as shown in Fig. 5.2A+B. Due to the egg laying deficiency of the mutants, the eggs reside longer in the uterus than in wild type nematodes so that eggs of later developmental stages can be found in utero. In wild type worms, eggs reside in the uterus for approximately 150 minutes until they have reached approximately 30 cell stage (Hirsh et al., 1976). The embryonic development then continues outside of the uterus until eventually L1 larvae are hatching about $14 \mathrm{~h}$ post-fertilization (Sulston et al., 1983). In the catp-6 mutants in contrast, eggs are retained in the uterus so that the embryonic development continues inside the hermaphrodite parent and - if the Egl phenotype is severe - the larvae eventually even hatch inside their parent which results in a "bag-of-worms" phenotype that leads to death of the parent. The egg laying defect in catp- 6 mutants is accompanied by morphological abnormalities of the vulva namely a protruding vulva (pVul) phenotype as depicted in Fig. 5.2C. pVul phenotypes mostly result from failures of the vulva tissue to connect properly to the uterus during development, which is normally important to allow for the passage and delivery of fertilized eggs. Vulva-uterine connection defects can at least in part explain the observed egg laying defect. 


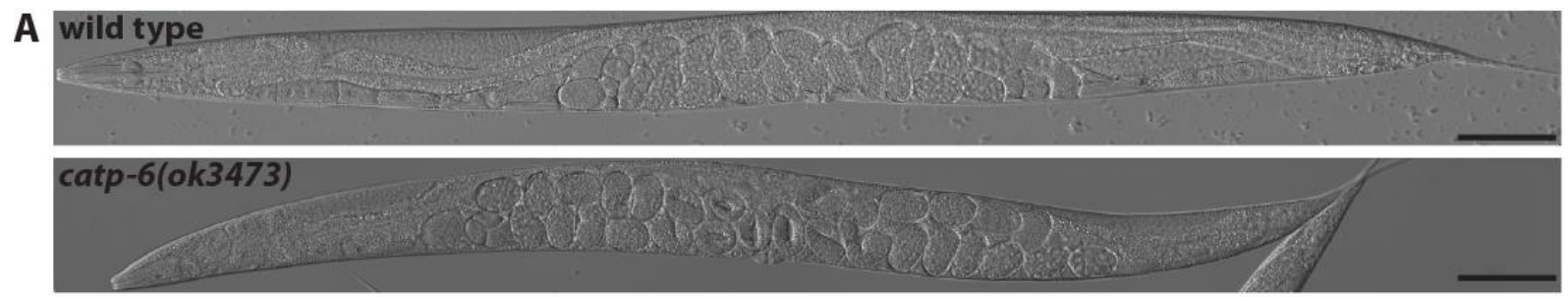

B
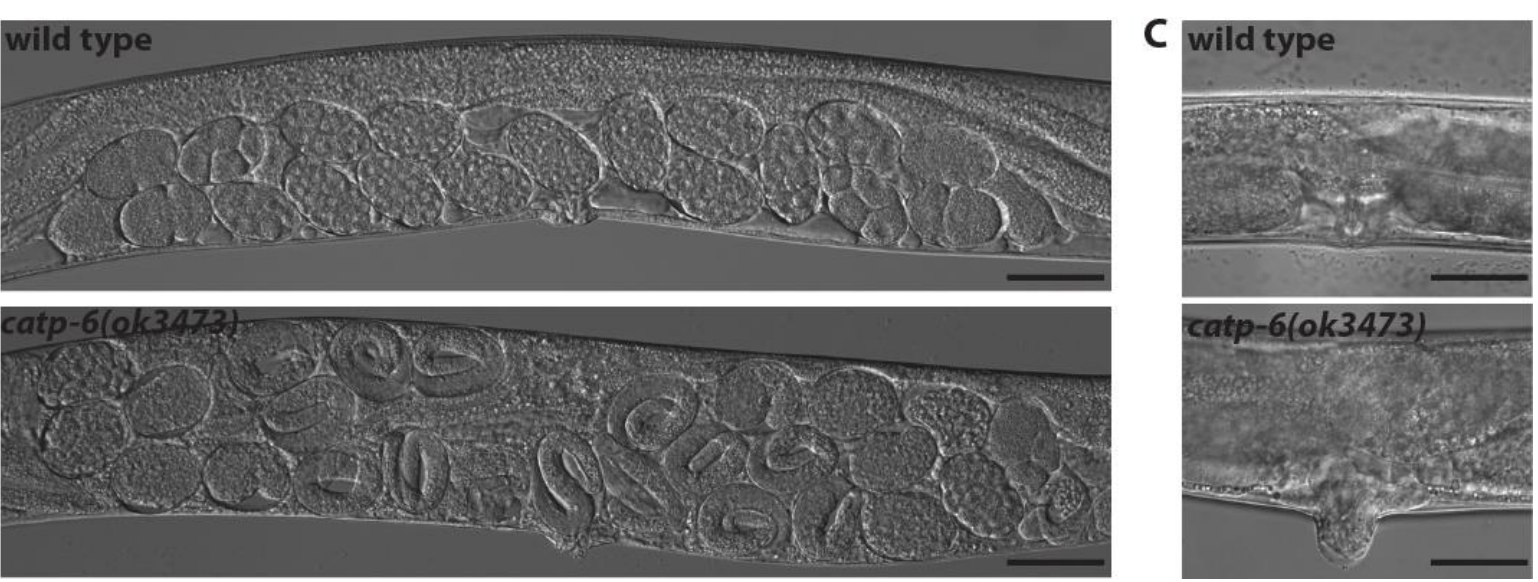

Fig. 5.2: catp-6 deletion mutants have an egg-laying defect and protruding vulva phenotype.

(A-C) DIC pictures of wild type and catp-6 worms. (A) DIC pictures of whole worms were taken from adults $24 \mathrm{~h}$ post-L4 stage. Scale bars represent $100 \mu \mathrm{m}$. (B) Pictures of the uterus were taken from adults $48 \mathrm{~h}$ postL4 stage. catp- 6 worms are Egl (egg laying defective) and accumulate eggs in the uterus. Therefore, eggs of later developmental stages are seen in the uterus. Scale bars represent $50 \mu \mathrm{m}$. (C) Pictures of the vulva were taken from young adult worms and reveal that catp- 6 worms show a pVul (protruding vulva) phenotype. Scale bars represent $50 \mu \mathrm{m}$.

After identifying Egl and pVul phenotypes in catp- 6 deletion mutants we decided to determine the brood size of catp- 6 mutants, which was done by our research technician Dr. Sabine König. While wild type $C$. elegans hermaphrodites on average give rise to around 300 progeny $(278 \pm 5)$, the brood size of the deletion mutants catp-6(tm3190) and catp-6(ok3473) was $38 \pm 40$ and $92 \pm 42$, respectively, meaning a reduction of the brood size by $60 \%$ in the case of catp-6(tm3190) and 85\% in the case of catp-6(ok3473) (Table 5.1).

Table 5.1: Mutations in catp-6 cause Egl and pVul defects and reduced brood size.

\begin{tabular}{ccccccc}
\hline \multicolumn{1}{c}{ Genotype } & $\begin{array}{c}\% \\
\text { non Egl }\end{array}$ & $\begin{array}{c}\% \text { Egl } \\
\text { partial }\end{array}$ & $\begin{array}{c}\% \text { Egl } \\
\text { strong } \\
(\mathrm{pVul})\end{array}$ & $\%$ sterile & $\mathrm{N}$ & brood size \\
\hline wild type $\mathrm{N} 2$ & 100 & 0 & 0 & 0 & 10 & $278 \pm 5$ \\
catp-6(ok3473) & 0 & 14 & 68 & 18 & 22 & $38 \pm 40$ \\
catp-6(tm3190) & 25 & 17 & 58 & 0 & 24 & $92 \pm 42$ \\
\hline
\end{tabular}


In addition to the Egl and pVul phenotypes, we also detected that catp- 6 mutations can lead to low levels of sterility. $18 \%$ of catp-6(ok3473) worms were sterile while none of the catp-6(tm3190) mutants or wild type worms were found to be sterile. Around $15 \%$ of both catp-6 mutants were exhibiting a partial Egl phenotype and another $60-70 \%$ of these mutants show strong Egl and pVul phenotypes. This indicates that catp- 6 plays a role for the development of the $C$. elegans reproductive tissues and that loss of function of catp- 6 results in morphological and functional defects in the reproductive apparatus leading to severely reduced brood sizes.

\subsubsection{Postembryonic development is delayed in catp-6}

When culturing catp- 6 mutants we observed that they develop more slowly and reach adulthood at later time points. Therefore, we were quantifying the time of postembryonic development (PED) by determining how long it took freshly laid eggs to develop to adults capable of producing eggs themselves. In order to avoid effects of a delayed embryonic development, only worms that had reached the L1 stage 12 hours after the eggs had been laid were subjected to the analysis. For wild type worms it took $69 \pm 5 \mathrm{~h}$ (mean $\pm S D$ ) to reach adulthood and a significant delay of the PED development was seen in both catp- 6 deletion strains which were reaching adulthood after $98 \pm 6$ and $92 \pm 11 \mathrm{~h}$ (Fig. 5.3). Besides the delayed PED in catp-6, we also observed that the development of the mutants was very asynchronous with some few worms reaching adulthood after 72 and $84 \mathrm{~h}$ for catp-6(tm3190) and catp-6(ok3473), respectively, while the slowest growing worms became adults only after 120 and $144 \mathrm{~h}$. Notably $6 \%$ of the catp-6 larvae were arresting at the L1 stage and did not develop any further. This suggests that loss of function of catp-6 not only slows down PED in C. elegans but to some extent even causes lethality at early larval stages.

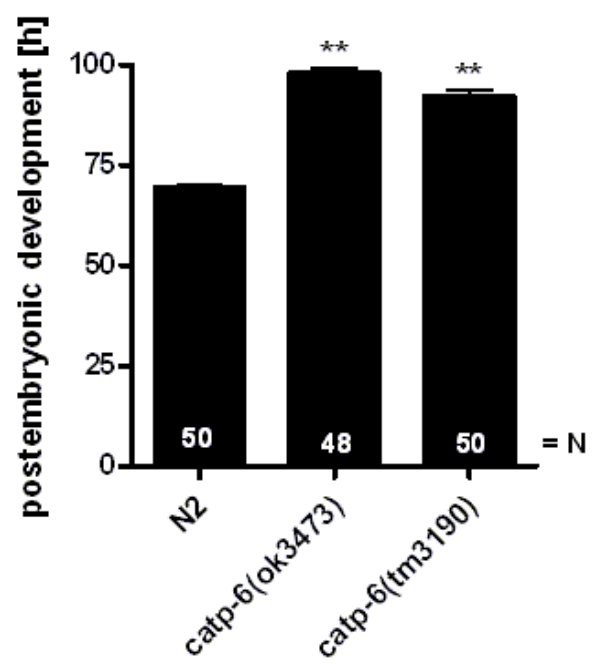

Fig. 5.3: Postembryonic development is delayed in catp-6 deletion mutants.

Postembryonic development was assessed by determining the time required for freshly laid eggs to develop to adulthood. It is delayed in both catp- 6 alleles. The error bars correspond to the standard error of the mean (SEM) and the significance values of one way ANOVA test with Dunnet's multiple comparison analysis are indicated: ${ }^{* *} \mathrm{P}<0.01$. 


\subsection{4. catp-6 mutants display locomotion defects due to muscular dysfunction}

When raised on plates with bacterial food, catp- 6 animals appeared lethargic as compared to wild type. Thus, we were assessing the locomotion of catp- 6 mutant animals. C. elegans locomotion is characterized by sinusoidal movements of the worm and can be quantified by determining the number of body bends per minute with one body bend corresponding to a completed sinusoidal wave. Movement analysis in young adult $C$. elegans revealed that locomotion is impaired in catp-6 (Fig. 5.4A). While the number of body bends per minute is 21 for wild type, it is reduced by about $30 \%$ to 14 in the mutants.

A

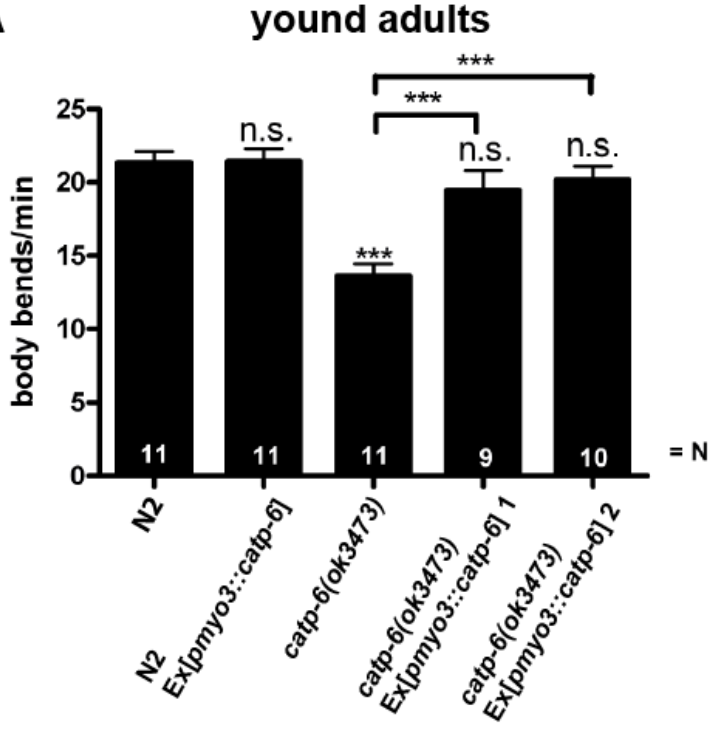

B

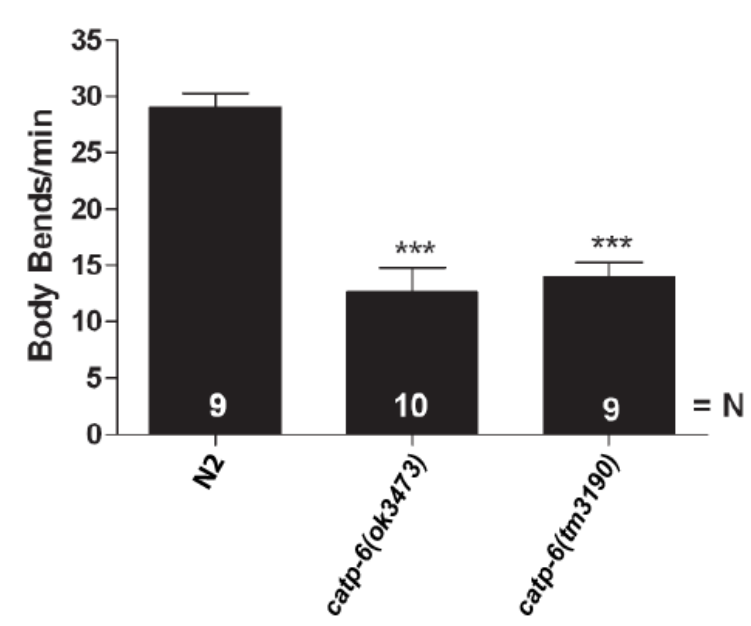

Fig. 5.4: catp-6 worms have a movement defect which can be rescued by expression of catp-6 in muscle cells and which is already apparent at larval stages.

(A) Movement is significantly reduced in young adult catp-6 deletion mutants. This effect can be rescued by expression of wild type catp-6 from a muscle-specific myo-3 promoter. Error bars correspond to the standard error of the mean (SEM) and significance values of one way ANOVA with Tukey's multiple comparison analysis are indicated: ${ }^{* *} P<0.001$; ns $P>0.05$. (B) A significant movement defect of catp- 6 deletion mutations is already present at larval stages. Error bars correspond to the standard error of the mean (SEM) and significance values of one way ANOVA with Dunnett's multiple comparison analysis are indicated: ${ }^{*} \mathrm{P}<0.01$.

The movement defect in catp- 6 could result either from defects in morphology or function of body wall muscles or from defects in neurotransmission from the presynaptic site. In order to distinguish between these two possibilities we tried to rescue the locomotion defects by expressing the wild type catp- 6 cDNA under control of the muscle-specific myo-3 promoter. If reduced locomotion is caused by muscular dysfunction due to lack of functional CATP-6, it should be possible to rescue the defect by restoring CATP- 6 function specifically in the body wall muscles (BWMs). In order to exclude that overexpression of catp-6 already has an influence on locomotion we first generated a transgenic line expressing catp- 6 from a muscle- 
specific myo-3 promoter in the wild type background. As shown in Fig. 5.4A, no change in locomotory rates was observed in these worms when compared to wild type. The extrachromosomal array containing the rescue construct myo-3::catp-6 was then crossed into the catp-6(ok3473) deletion mutant and locomotion was restored to wild type levels (20 and 18 body bends per minute). This indicates that indeed the locomotion defect of catp- 6 is caused by a muscular requirement for CATP- 6 function. In order to assess whether this muscular defect is resulting from a degenerative process with increasing age we decided to perform a movement assay on L3 larvae. This experiment was performed together with Victor Bustos, a lab rotation student in the lab. As shown in Fig. 5.4B the movement defect of both catp-6 mutants is already apparent at this larval stage indicating that loss of catp- 6 in BWMs causes developmental defects in muscles rather than muscular degeneration in an age-dependent manner.

\subsection{5. catp-6 is expressed in multiple tissues}

To further understand which tissues in C. elegans require CATP-6 function, we determined the catp- 6 expression pattern. For this purpose, GFP was expressed under the control of a $4 \mathrm{~kb}$ catp- 6 promoter fragment ranging from the Start ATG to position $-4 \mathrm{~kb}$. Cloning of this construct was performed by Katrin Schwarze, a former technician of our lab. The expression of this reporter construct was very weak but could be observed in the pharynx, nerve ring as well as in some structures in the tail. High expression was furthermore seen in body wall muscles (BWMs) and in the vulva tissue. catp-6 expression in muscles and vulval tissues fits well with the Egl and pVul phenotypes and with the impaired movement of catp- 6 deletion mutants. Given that the expression of the reporter construct was very weak, it is likely that we were only detecting expression in tissues which are expressing catp- 6 at high levels and that catp- 6 is expressed in other tissues as well. 


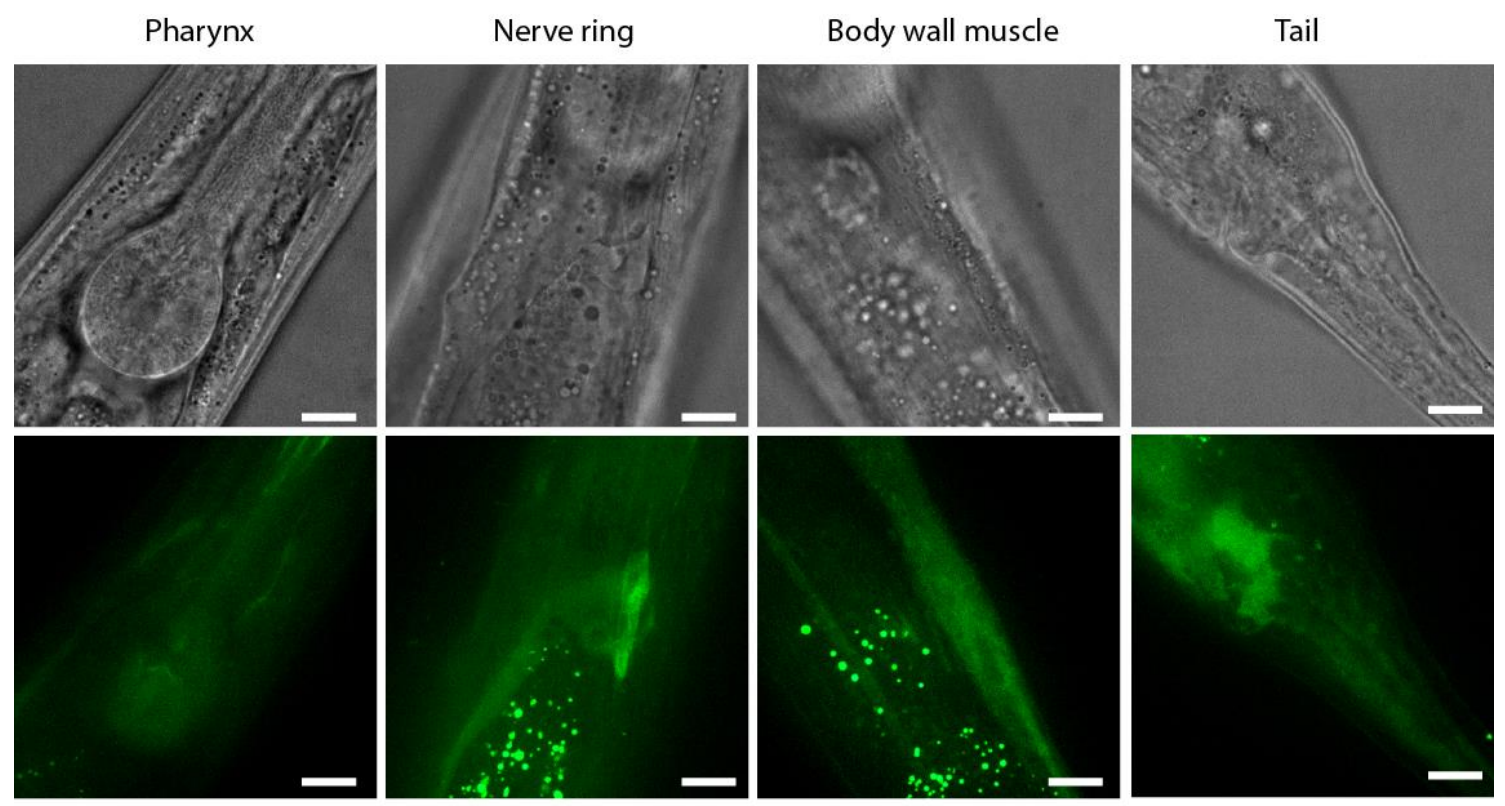

Vulva

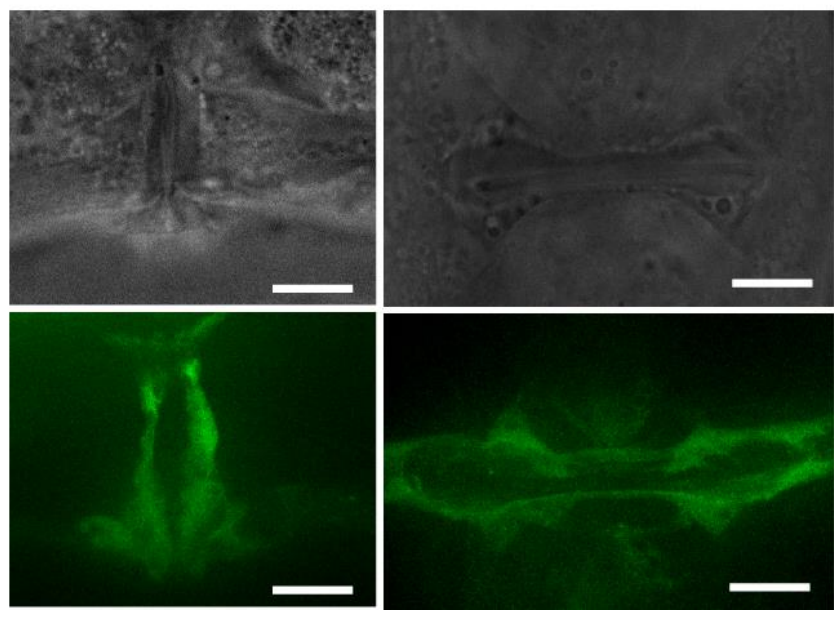

Fig. 5.5: catp-6 expression pattern.

For expression pattern analysis, a transcriptional reporter was used. GFP expression driven by the catp- 6 promoter is visible in pharynx, nerve ring, tail as well as in BWM cells and in the vulva. Scale bars represent $10 \mu \mathrm{m}$.

\subsubsection{The structure of muscle fibers is normal in catp-6}

Since we showed that catp-6 expression in BWMs is required for normal locomotion, we wanted to determine whether lack of CATP-6 function leads to morphological defects in BWMs. Therefore, we analyzed whether the organization of myofilaments is changed in catp-6 mutants. For this purpose, we used a translational fusion of GFP with MYO-3, a myosin heavy chain protein, as marker for thick filaments in BWMs. This experiment was performed by Dr. Sabine König, research technician in our lab. As depicted in Fig. 5.6, no differences in the myofilament structures between catp- 6 mutants and wild type could be ob- 
served, indicating that other structures in the muscle cells are likely to be affected by CATP-6 loss-of-function.

\section{Wild type}

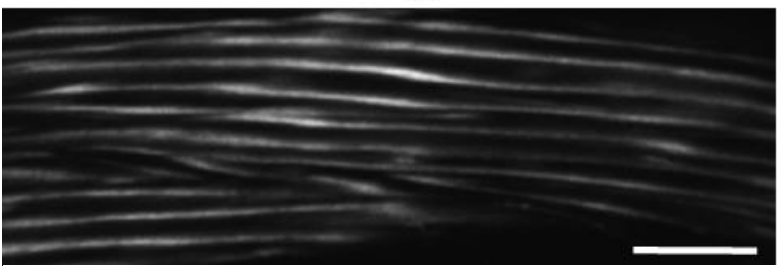

catp-6 (ok3473)

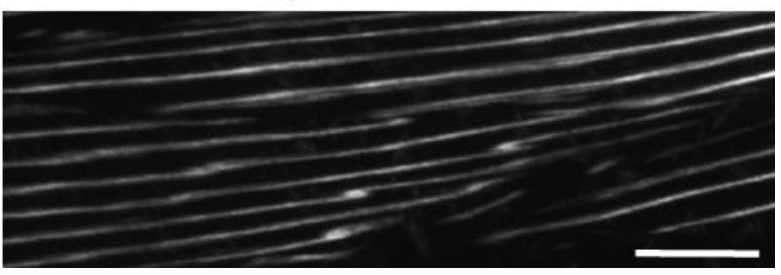

Fig. 5.6: Myofilament organization in BWM cells is not affected in catp-6 deletion mutants.

Thick filaments in C. elegans BWMs were visualized using a MYO-3-GFP fusion. The myofilament structure is not affected by loss of function of CATP-6. Scale bars represent $10 \mu \mathrm{m}$. Images were kindly provided by Dr. Sabine König.

\subsection{7. catp-6 mutants show vulva muscle defects}

The egg laying defect of catp- 6 mutants indicates that the egg laying apparatus in the mutants is disturbed. As several structures are involved in the egg laying process, we were interested in understanding which components are affected by loss of catp- 6 . A scheme of the C. elegans egg laying apparatus is depicted in Fig. 5.7. First of all, vulva and uterus are essential for egg laying and the establishment of the vulva-uterine connection also plays a critical role (Newman and Sternberg, 1996). Furthermore, the sex muscles, which comprise eight vulval and eight uterine muscles, are very important as their contraction leads to opening of the vulva and contraction of the uterus thus promoting egg laying [reviewed in (Schafer, 2005)]. Finally, innervation of the sex muscles by two hermaphrodite specific neurons (HSN) and six ventral cord (VC) motoneurons is a prerequisite for proper egg laying [reviewed in (Schafer, 2005)].

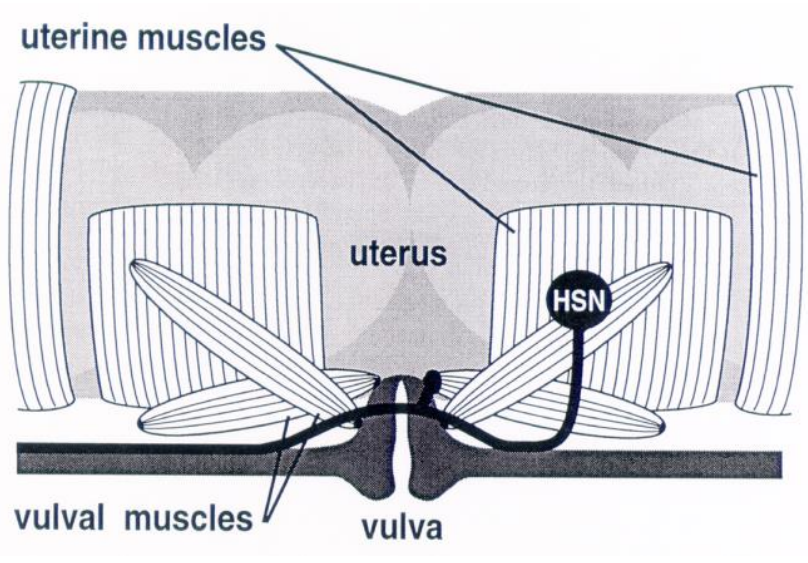

Fig. 5.7: C. elegans egg laying apparatus.

Several structures are involved in the egg laying process. Besides the uterus and the vulva, the vulva and uterine muscles as well as innervation by the HSN neurons are essential parts of the egg laying system. Reprinted by permission from Cold Spring Harbor Laboratory Press: Cold Spring Harbor Symposia on Quantitative Biology V.57, Clark et al., copyright (1992). doi:10.1101/SQB.1992.057.01.041 (Clark et al., 1992). 
A large part of the Egl phenotype of catp- 6 mutants most likely arises from defects in the vulva-uterine connection which in most cases leads to a pVul phenotype. However, in addition, $14 \%$ and $17 \%$ of the catp-6(ok3473) and catp-6 (tm3190) mutants, respectively, are Egl without displaying a pVul phenotype. This strongly suggests that there might be additional defects within the egg laying system that are contributing to the Egl defect. Based on the requirement of CATP- 6 activity in the BWMs for proper locomotion we reasoned that catp- 6 animals might also have defective sex muscles. It has been shown that a set of eight vulva muscles is strictly required for normal egg laying and vulva muscles have been reported to be more important for the egg laying process than the uterine muscles [reviewed in (Schafer, 2005)]. Cases in which vulva muscles are partly missing or misaligned and improperly attached to the vulva cells lead to defects in egg laying (Eimer et al., 2002). To determine whether part of the Egl defect of catp- 6 mutant animals is caused by defective sex muscles, we visualized the sex muscles by GFP. The ceh-24 promoter drives expression of GFP in the eight vulval muscles. In wild type worms, the eight vulva muscles visualized by ceh-24::gfp expression are arranged around the vulva opening as two $x$-shaped sets of four mononucleated smooth muscles, termed vm1 and vm2 (Fig. 5.8A). When imaged from the ventral side, the two $x$-shaped sets of vulva muscles are laying on top of each other and the nuclei of the eight vulva muscles are clearly visible by their strong accumulation of GFP fluorescence.
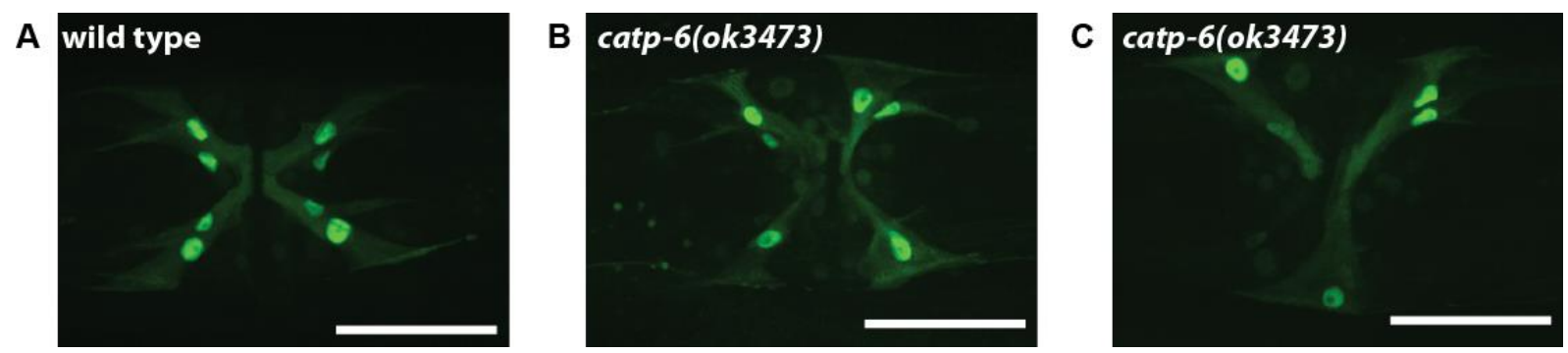

Fig. 5.8: Vulva muscle defects in catp-6 deletion mutants.

Vulva muscles were imaged in worms expressing ceh-24::gfp. The vulva is depicted from a ventral view and the nuclei of the muscle cells appear as bright spots. In wild type worms, the eight vulva muscles are regularly arranged in cross-shape (A). In catp-6 mutants, several defects of the muscles can be observed. These include defects in the attachment of the muscles to the vulva and irregular muscle arrangement (B) as well as lack of at least one of the eight vulva muscles (C). Scale bars represent $20 \mu \mathrm{m}$.

As depicted in Figure 5.8A, the muscles are very regularly and symmetrically arranged in wild type worms and the muscles are well attached to the vulva enabling opening of the vulva upon contraction. In contrast, an irregular arrangement of the vulva muscles is seen in $36 \%$ of the catp-6 mutants (Fig. 5.8B; Table 5.2). Furthermore, in 10\% of the mutant worms, the vulva muscles are not properly attached to the vulva (Fig. 5.8B; Table 5.2) and, most remarkably, in more than $50 \%$ of the mutants at least one of the eight vulva muscles is missing (Fig. 5.8C; Table 5.2). 
Table 5.2: Vulva muscle defects in catp-6.

\begin{tabular}{lcccc}
\hline Genotype & $\begin{array}{c}\text { \% Muscles not } \\
\text { properly attached } \\
\text { to vulva }\end{array}$ & $\begin{array}{c}\text { \% Irregular } \\
\text { muscle arrange- } \\
\text { ment }\end{array}$ & $\begin{array}{c}\text { \% Lacking at least } \\
\text { one vulva muscle }\end{array}$ & $\mathrm{N}$ \\
\hline wild type $\mathrm{N} 2$ & 0 & 0 & 4 & 25 \\
catp-6(ok3473) & 10 & 38 & 52 & 27 \\
\hline
\end{tabular}

The vulva muscle defects together with the pVul phenotype, which is indicative of a defective vulva-uterine connection, might be the reason for highly penetrant egg-laying defects present in catp- 6 deletion mutants. As the Egl phenotype of catp- 6 worms is quite strong and as we have not checked for changes in other components of the egg laying apparatus we cannot exclude that additional defects in uterine muscles, the structure of the vulva or problems with neuronal input from HSN or VC also have minor contributions to impaired egg laying in catp-6 mutants.

\subsubsection{Mitochondrial morphology is severely affected in muscle cells of catp-6 mutants}

The defects in the vulva muscles as well as the impaired movement of catp- 6 mutants that was also resulting from muscular defects brought us to investigate the BWM cells on a cellular level. Since we had observed severe changes in mitochondrial morphology in muscle cells upon expression of $\alpha S$ as described in Chapter I and given the reported functional link between aS and CATP-6 (Gitler et al., 2009), we decided to analyze mitochondrial morphology in catp- 6 mutants. Mitochondria in catp- 6 mutants were severely affected, with $60 \%$ of the cells showing networks of very long and thin mitochondria. The mitochondria in these cells were also more interconnected than in wild type muscles and showed an irregular arrangement with condensed mitochondrial structures around the nucleus. In contrast, mitochondria in wild type worms were very regularly arranged, forming tubular structures in which an intraluminal space was clearly visible. In order to determine whether loss of function of catp- 6 was indeed responsible for the changes in mitochondrial dynamics of the mutants, we tried to rescue the phenotypes by expressing the wild type catp- 6 cDNA in the BWM muscle cells. First, we created transgenic worms expressing catp-6 from a muscle-specific myo-3 promoter in a wild type background. This extrachromosomal array was able to rescue the locomotion defects of catp- 6 mutants but did not cause any locomotion defects when expressed in wild type animals in addition to endogenous catp-6 (Fig. 5.4). In agreement with this, most of the cells in these transgenic animals displayed wild type-like mitochondrial mor- 
phology and only $15 \%$ were showing slightly elongated mitochondria, indicating that weak overexpression of catp- 6 does not induce severe changes in mitochondrial dynamics.

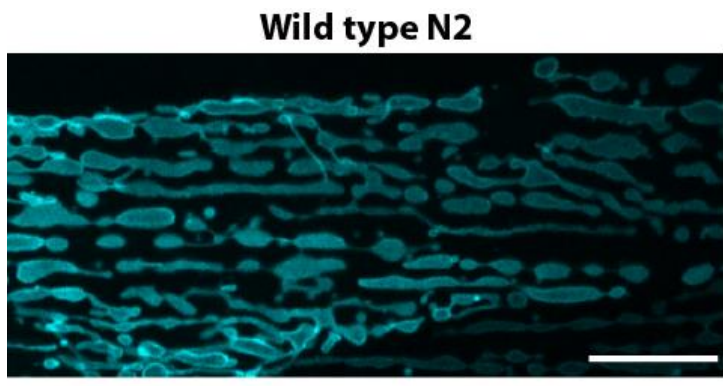

N2 Ex[pmyo-3::catp-6]
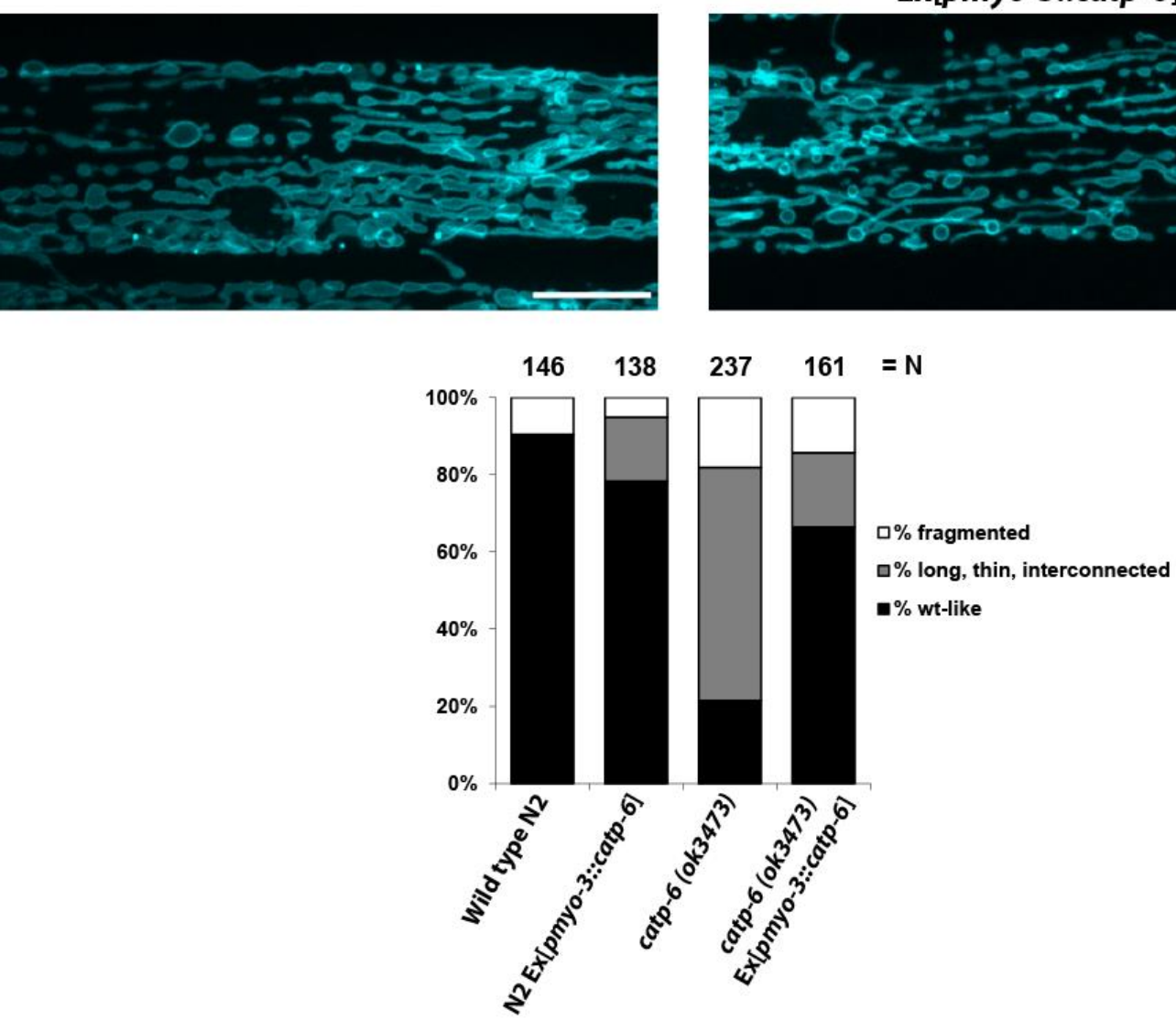

catp-6 (ok3473)

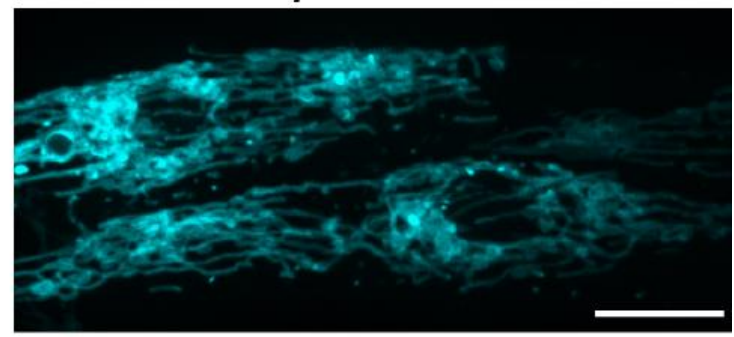

catp-6 (ok3473)

Ex[pmyo-3::catp-6]

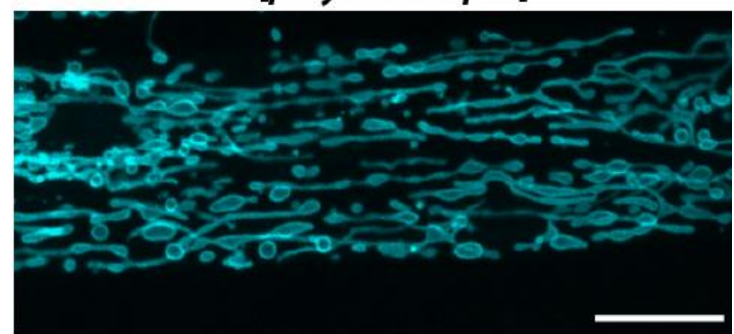

$\square \%$ long, thin, interconnected

(1)

Fig. 5.9: Mitochondrial elongation and perinuclear clustering in catp-6 deletion mutants.

Mitochondria in BWM of young adult worms were visualized by expressing CFP targeted to the outer mitochondrial membrane by fusion to the TM domain of yeast TOM70. Mitochondria in wild type worms form regularly arranged tubules. In catp- 6 mutants, mitochondria are longer, thinner and more interconnected and are clustering around the nucleus. Overexpression of catp- 6 in muscle cells of wild type worms does not induce severe changes of mitochondrial morphology, but expression of catp-6 in muscle cells of the deletion mutants rescues the mitochondrial morphology defects. Scale bars represent $10 \mu \mathrm{m}$. Quantification of mitochondrial morphology was done by determining the relative occurrence of "wild type-like", "long, thin and interconnected" as well as "fragmented" mitochondria. The numbers of muscle cells analyzed are indicated above the bar graph. 
Next, we crossed these worms expressing catp- 6 only in BWM cells into the catp- 6 mutant background and found that indeed, mitochondrial morphology is rescued by restoring CATP-6 function specifically in BWM cells. More than $60 \%$ of the rescued BWM cells displayed now a wild type-like morphology. Thus, we could clearly show that loss of function of catp- 6 causes mitochondrial elongation, network formation and perinuclear clustering of the mitochondria.

\subsubsection{Mitochondrial morphology is already affected at larval stages in catp-6}

Having seen the defects in mitochondrial morphology in adult catp- 6 mutants, we sought to determine whether they are already present at larval stages as we had observed that movement was already affected in L3 larvae of catp-6. Therefore, we imaged mitochondria in BWMs of L3 larvae. As depicted in Fig. 5.10, mitochondrial clustering around the nucleus can already be observed at this larval stage. As already seen in the adult worms, long and very thin mitochondria are the predominant mitochondrial structures and are seen in almost $60 \%$ of the cells while only $20 \%$ of cells show wild-type like mitochondrial morphology. This suggests that mitochondrial morphology is already affected by loss of function of catp- 6 in developmental stages and is not due to a degenerative process which occurs during aging.

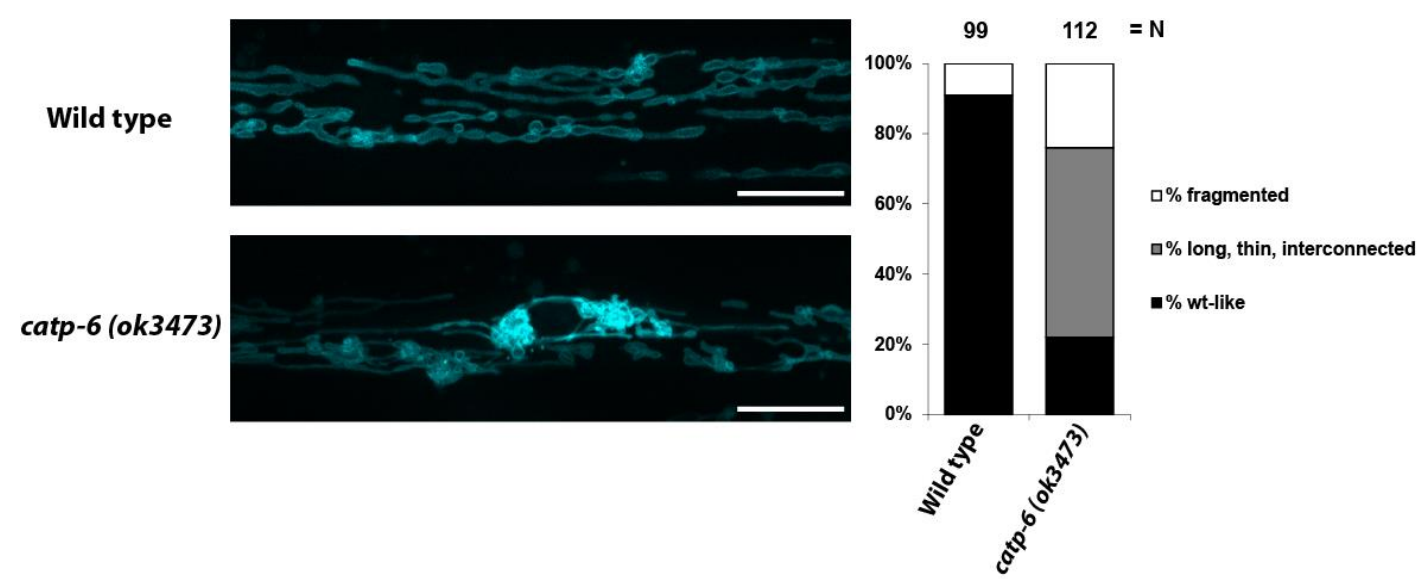

Fig. 5.10: Changes in mitochondrial morphology and perinuclear clustering are already present in L3 larvae of catp-6 mutants.

Mitochondrial outer membrane in BWM cells of L3 larvae was visualized by expression of the previously described pmyo-3::tom70-cfp construct. Already at this larval stage, mitochondria of catp-6 mutants are longer and thinner than in wild type and are accumulating in close proximity of the nucleus. Scale bars represent $10 \mu \mathrm{m}$. Quantification of mitochondrial morphology was done by determining the relative occurrence of "wild type-like", "long, thin and interconnected" as well as "fragmented" mitochondria. The numbers of muscle cells analyzed are indicated above the bar graph. 


\subsubsection{EM analysis reveals collapsing of mitochondria and loss of mitochon- drial cristae in catp- 6 mutants}

After having observed severe changes in mitochondrial morphology and arrangement by confocal microscopy, we decided to investigate these changes on an ultra-structural level by High-Pressure-Freeze (HPF) Electron Microscopy (EM). EM analysis was performed by Dr. Jan Hegermann, a former postdoc of our lab.

A

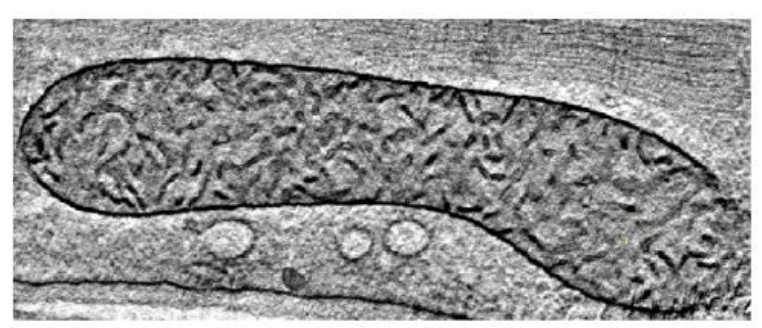

Wild type

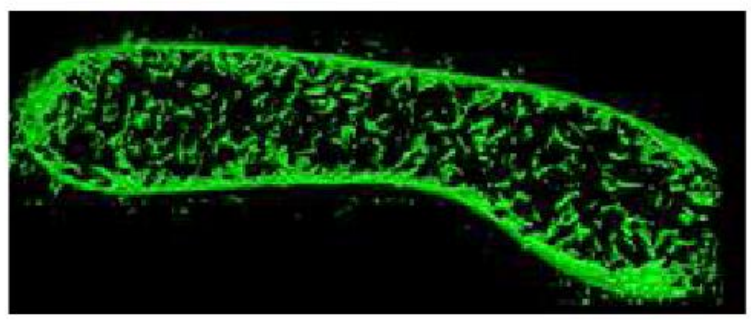

catp-6 (ok3473)
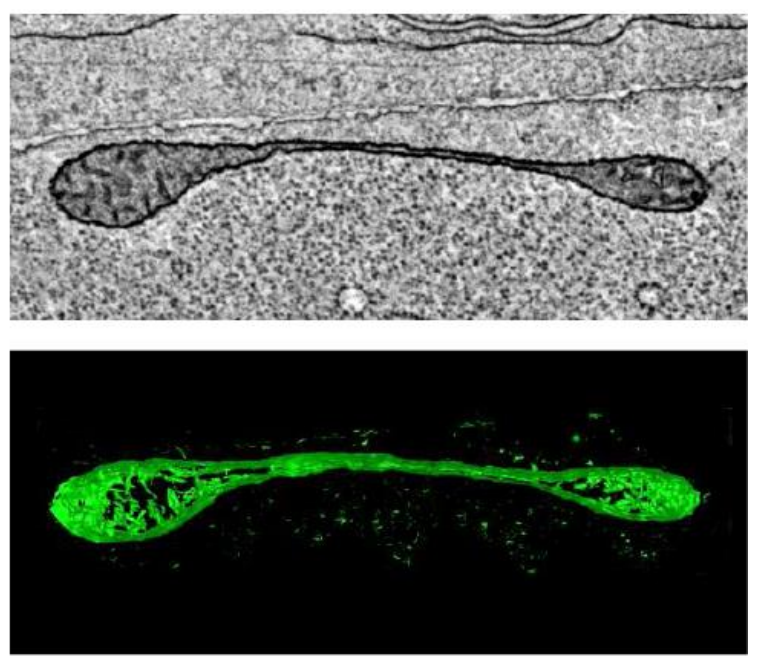

B

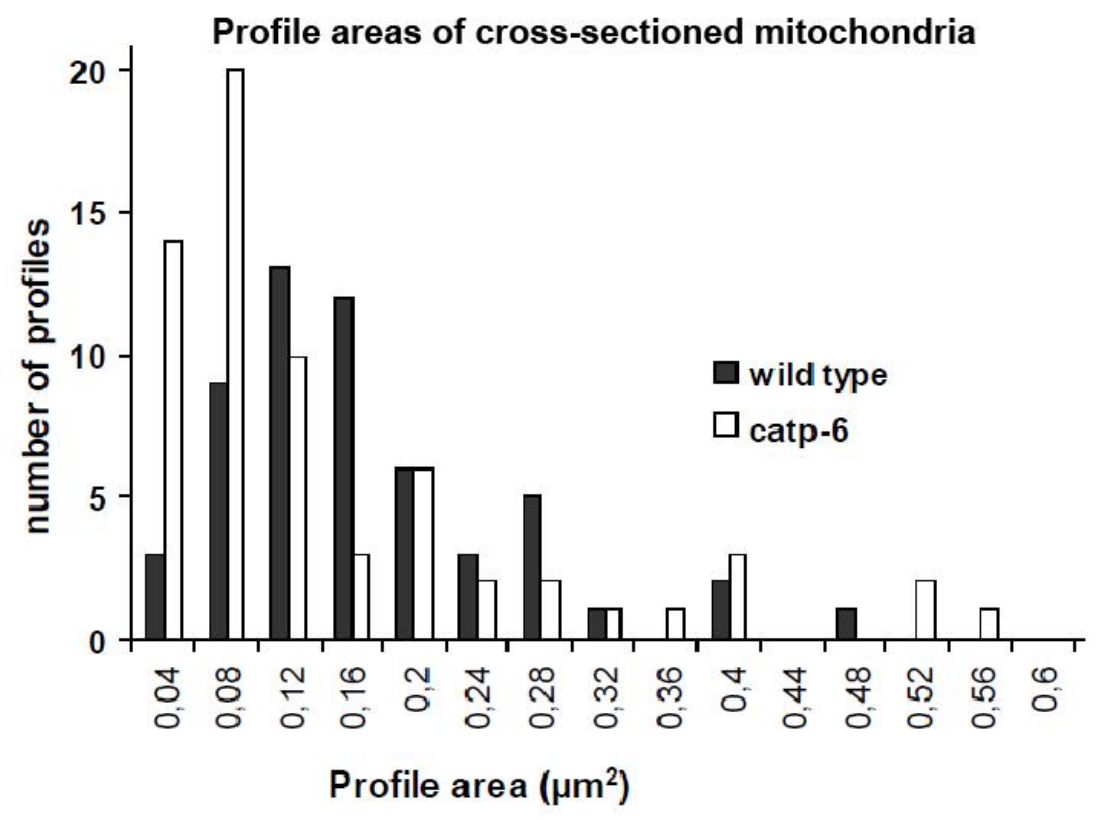

Fig. 5.11: EM analysis of mitochondria in BWMs of catp-6 mutants.

(A) Tomograms of mitochondria in longitudinal sections of BWM cells of young adult wild type and catp-6 worms. Mitochondria in catp- 6 mutants are collapsed in the middle so that the membranes are coming in direct contact with each other. Cristae are lacking in those collapsed regions. (B) Histogram of mitochondrial profile areas in cross-sections through BWM cells of young adult worms. In catp-6 deletion mutants, smaller mitochondrial structures are seen in the cross sections when compared to wild type. Pictures were kindly provided by Dr. Jan Hegermann. 
Tomograms of mitochondria in longitudinal sections of BWM cells revealed that mitochondria in catp- 6 mutants are collapsed in the middle with the two inner membranes coming in direct contact to each other without any luminal space separating them (Fig. 5.11A). The collapsed regions are completely devoid of cristae structures. It needs to be determined whether loss of cristae is causative for the collapse.

To get an estimate of the diameter of mitochondria in wild type worms and catp-6 mutants, the profile area of cross-sectioned mitochondria was analyzed. The histogram of the profile areas of mitochondria in cross sections of BWM cells is shown in Fig. 5.11B. This distribution was clearly shifted to smaller sizes in catp- 6 mutant animals as compared to wild type. While in wild type worms most of the mitochondrial profile areas were between 0.12 and $0.16 \mu \mathrm{m}^{2}$, the most abundant profile areas in catp- 6 mutants were between 0.04 and $0.08 \mu \mathrm{m}^{2}$. Given the fact that no cristae are present at collapsed regions of the mitochondria, cross sections through these regions might not be identified as mitochondria. Therefore, those very small profile areas are likely to be missed in the profile area analysis. The results of the HPF-EM analysis of mitochondria in muscle cells of catp- 6 deletion mutants fit nicely to the changes in mitochondrial morphology that were seen in the fluorescence images and indicate that CATP-6 plays a role in maintaining mitochondrial cristae structure and morphology.

\subsubsection{The mitochondrial fission protein DRP-1 is clustering in close proximity to the nucleus in BWM cells of catp- 6 mutants}

As we had observed that mitochondria in catp-6 mutants are longer, thinner and form a highly interconnected network in contrast to those of wild type muscles, we wanted to understand which mechanism mediates these changes in mitochondrial morphology. Mitochondria are very dynamic organelles that are steadily fusing and dividing [reviewed in (Bereiter-Hahn and Voth, 1994; Detmer and Chan, 2007; Westermann, 2010b)]: Mitochondrial morphology varies between different cell types and can be adjusted to the cellular status [reviewed in (Kuznetsov et al., 2009; Sauvanet et al., 2010)]. A well-adjusted balance between fission and fusion is very important for mitochondrial function and several proteins are coordinating mitochondrial dynamics [reviewed in (Detmer and Chan, 2007; Westermann, 2010b)]. DRP-1 (Dynamin Related Protein 1) plays a crucial role for mitochondrial fission (Smirnova et al., 2001). Cytosolic DRP-1 can associate to mitochondria and oligomerization at the mitochondrial outer membrane leads to formation of ring-like structures around the mitochondrial tubules. GTP hydrolysis then induces scission of the mitochondrial membranes and thus leads to mitochondrial fission. Loss of DRP-1 function inhibits the fission of the mitochondrial outer membrane resulting in the occurrence of elongated mitochondria. Thus, inactivation of 
DRP-1 shifts the balance between mitochondrial fission and fusion towards fusion. Furthermore, clustering of highly interconnected mitochondrial tubules around the nucleus was described in cells expressing dominant negative DRP-1 (Smirnova et al., 2001; Smirnova et al., 1998). As these mitochondrial phenotypes observed in cells lacking functional DRP-1 strongly resemble the mitochondrial morphology that we observed in catp-6 mutants, we decided to have a closer look at the distribution of the DRP-1 protein in catp- 6 mutants.

For this purpose, we generated a fusion protein of DRP-1 tagged with TagRFP at the $\mathrm{N}$-terminus and expressed this construct along with the previously described TOM70-CFP marker for the mitochondrial outer membrane in BWM cells. N-terminal fusion of a fluorescent protein to DRP-1 was done before in $C$. elegans and it was shown that the tag does not interfere with mitochondrial association of DRP-1 (Labrousse et al., 1999). Furthermore, the fusion protein seems to be functional as increased mitochondrial fragmentation was seen upon overexpression of tagged DRP-1 although the activity of the fusion protein seems to be reduced compared to untagged DRP-1 (Labrousse et al., 1999). As described previously (Labrousse et al., 1999), puncta of the TagRFP-DRP-1 fusion protein can be seen in wild type worms and these punctate structures are equally distributed throughout the cell (Fig. 5.12A). In contrast, large clusters of DRP-1 spots are seen in catp-6 deletion mutants. These DRP-1 clusters mostly are found in close proximity to the nucleus. Expression of wild type catp- 6 in muscle cells, which was able to rescue mitochondrial morphology as described in section 5.2.8 of this chapter, also restores normal TagRFP-DRP-1 distribution. Overexpression of catp- 6 in wild type worms does not affect DRP-1 localization and as previously described also does not induce severe changes in mitochondrial morphology.

Quantification of TagRFP-DRP-1 clusters as depicted in Fig. 5.12B reveals that large DRP-1 clusters close to the nucleus are seen in $89 \%$ of muscle cells in catp- 6 deletion mutants while they were almost never seen in wild type worms. Expression of catp- 6 under a muscle specific myo-3 promoter is sufficient to restore normal DRP-1 distribution decreasing the relative amount of observed DRP-1 clusters to $11 \%$ of muscle cells in catp- 6 mutants. This demonstrates that loss of CATP-6 function causes the changes in DRP-1 distribution. Since in catp-6 mutant animals the mitochondrial morphology resembles the one present in drp-1 mutants (Labrousse et al., 1999), it is likely that the observed DRP-1 clustering goes along with DRP-1 inactivation in catp- 6 mutants. Thus, loss of CATP-6 function probably disturbs proper function of DRP-1 and thereby induces mitochondrial elongation and perinuclear clustering. 
A

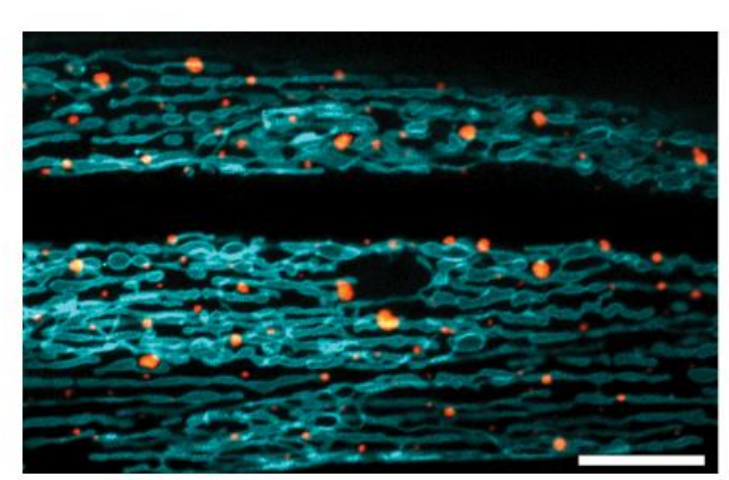

catp-6 (ok3473)

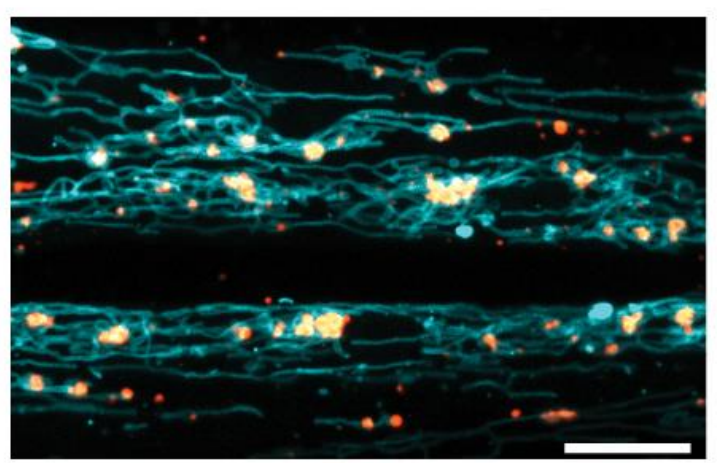

catp-6 (ok3473)

Ex[pmyo-3::catp-6]

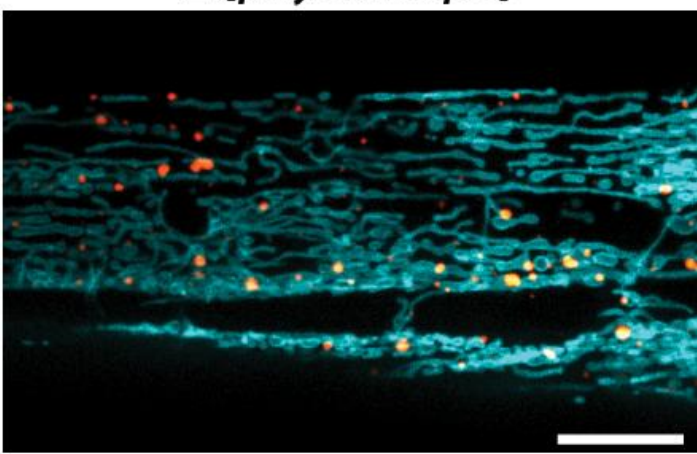

Wild type

Ex[pmyo-3::catp-6]

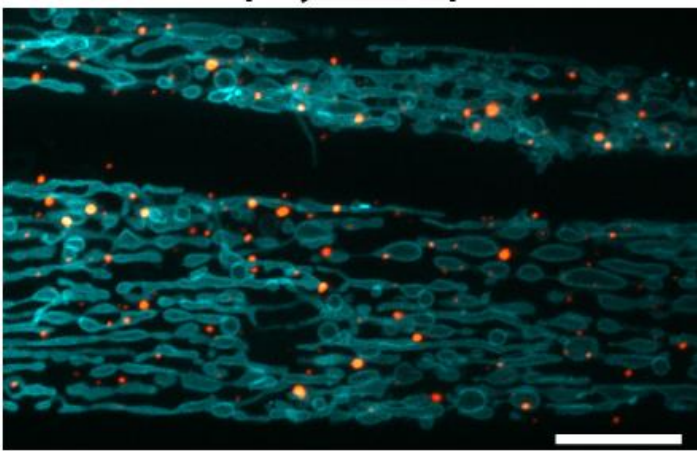

B

$\%$ cells with DRP-1 clusters

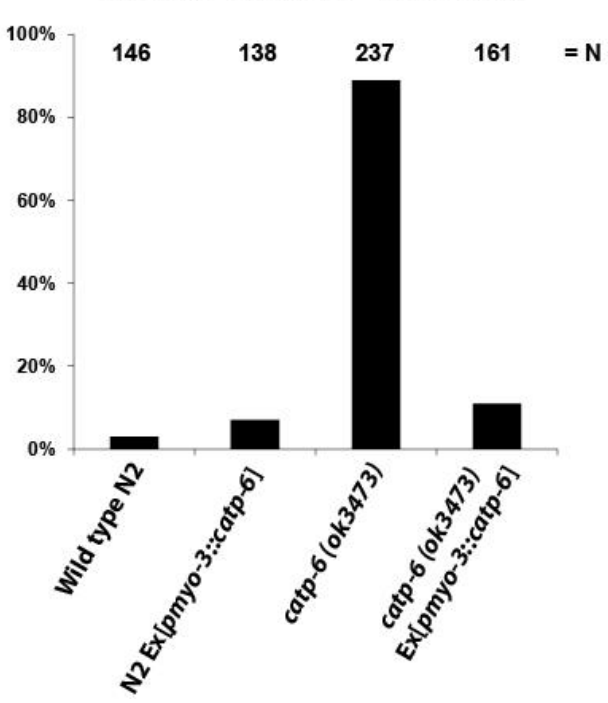

Fig. 5.12: Changes in mitochondrial morphology and distribution of the mitochondrial fission protein DRP- 1 in muscle cells of catp- 6 mutants.

(A) The mitochondrial fission protein DRP-1 was tagged N-terminally with TagRFP and expressed from a muscle-specific myo-3 promoter along with TOM70-CFP for visualization of mitochondria. In wild type cells, DRP-1 occurs as puncta that are equally distributed throughout the cell. In contrast, clustering of DRP-1 especially in close proximity to the nucleus was observed in catp-6. Expression of catp-6 in muscle cells of the deletion mutants restores normal DRP-1 distribution. Overexpression of catp-6 in wild type worms does not affect DRP-1 distribution. Scale bars represent $10 \mu \mathrm{m}$. (B) Quantification of DRP-1 clustering. Numbers of muscle cells analyzed are indicated above the bar graph. 


\subsubsection{RNAi against mitochondrial dynamics genes}

To get a more detailed understanding of how the balance between mitochondrial fission and fusion is affected by deletion of catp- 6 , we decided to investigate the impact of the individual mitochondrial dynamics genes by RNAi. The RNAi hypersensitive eri-1(mg366) strain was used as sensitized background for the RNAi experiments. We created transgenic worms expressing the mitochondrial marker TOM70-CFP and the TagRFP-DRP-1 fusion protein in muscle cells in order to see how silencing of the dynamics genes changes mitochondrial morphology and DRP-1 distribution in wild type worms and in the catp- 6 mutants. Four genes were chosen for analysis: two genes that are involved in mitochondrial fission ( $d r p-1$ and fis-1) and two genes that mediate mitochondrial fusion (fzo-1 and eat-3). $d r p-1$ is the C. elegans ortholog of mammalian Drp1 (Dynamin Related Protein 1) (Labrousse et al., 1999) and fis-1 is one of two orthologs of mammalian Fis1 in C. elegans. In the mammalian system, Fis 1 is involved in coordinating DRP-1 assembly at the mitochondria [reviewed in (Chen and Chan, 2004)]. Yet, whether this function is conserved in C. elegans remains to be determined (Westermann, 2010a). fzo-1 is the only C. elegans ortholog of the mammalian Mitofusins (Mfn1 and Mfn2) which mediate fusion of the mitochondrial outer membrane [reviewed in (Mozdy and Shaw, 2003; Westermann, 2010a)]. Inner membrane fusion in C. elegans is triggered by eat-3, the ortholog of mammalian Opa1 (Kanazawa et al., 2008).

Silencing of the fission gene drp-1 in wild type worms leads to the occurrence of mitochondrial blebs that are connected by very thin tubules (Fig. 5.13). The fact, that the RFP fluorescence signal of the TagRFP-DRP-1 fusion protein disappeared upon RNAi treatment against $d r p-1$, was reconfirming that the $d r p-1$ RNAi was working well. The structural changes that were observed upon knockdown (KD) of $d r p-1$ in wild type worms had been described in previous studies investigating drp-1 mutants (Labrousse et al., 1999) and performing RNAi experiments against $d r p-1$ in C. elegans muscle cells (Head et al., 2011) and it was shown that the bleb-like structures are filled with mitochondrial matrix indicating that fission of the inner mitochondrial membranes can still take place while the outer membranes cannot undergo fission upon drp-1 KD (Labrousse et al., 1999). Interestingly, mitochondrial morphology after silencing of $d r p-1$ resembles the elongated structures that we had observed in catp- 6 mutants but the bleb-like structures that were found upon drp- $1 \mathrm{KD}$ are absent in catp- 6 deletion mutants. Remarkably, KD of $d r p-1$ expression in catp- 6 deletion mutants also did not induce the occurrence of the bleb-like structures (Fig. 5.13). No gross changes in mitochondrial morphology were seen upon RNAi treatment against $d r p-1$ in the catp-6 mutants and mitochondrial elongation was still apparent. This suggests that DRP-1 function is largely absent in catp- 6 mutants. Therefore, it is likely that the DRP-1 clustering in catp- 6 mutants is a sign of DRP-1 inactivation in these mutants. 
eri-1(mg366)

L4440

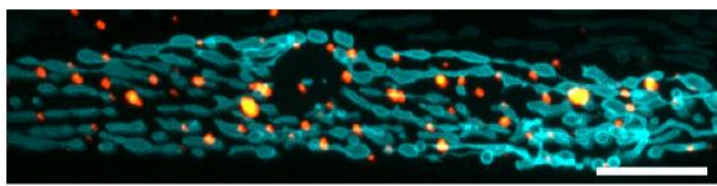

drp-1

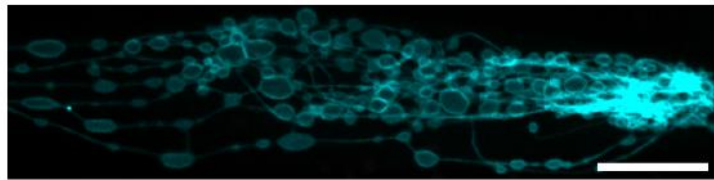

fis-1

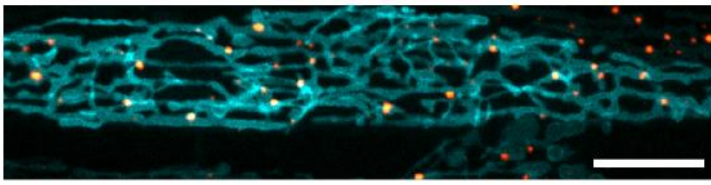

fzo-1

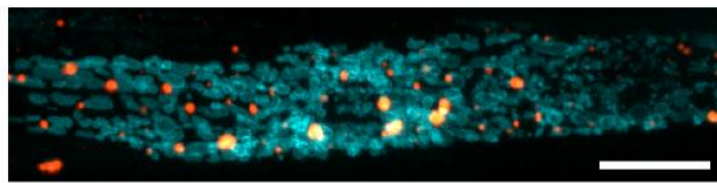

eat-3

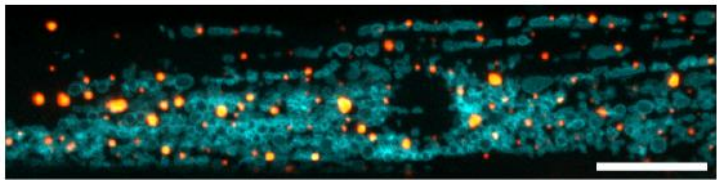

eri-1(mg366); catp-6(ok3473)
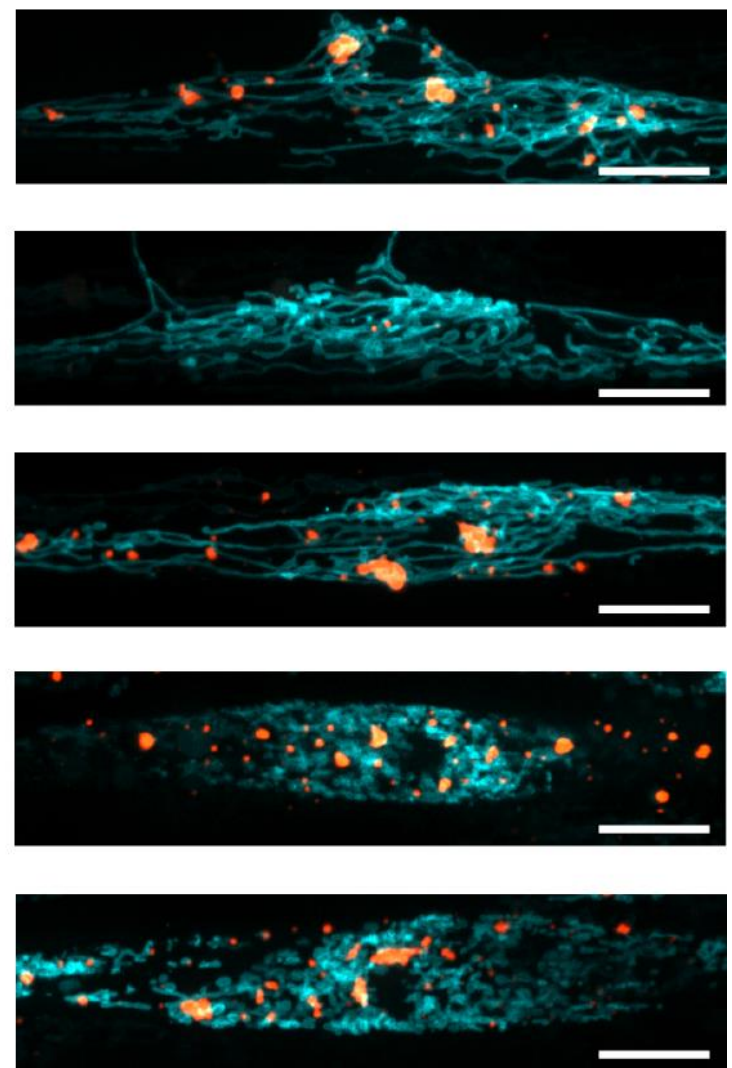

Fig. 5.13: Effect of silencing of mitochondrial dynamics genes on mitochondrial morphology in wild type worms and catp-6 mutants.

RNAi experiments were performed in an eri-1(mg366) which is a sensitized background for RNAi. The empty L4440 vector which was used as control does not lead to changes in mitochondrial morphology and DRP-1 distribution. TOM70-CFP was used as mitochondrial marker and TagRFP-DRP-1 was co-expressed along with this marker in BWM cells. Young adult worms were used for imaging. RNAi against the mitochondrial fission gene drp-1 leads to disappearance of the RFP signal indicating that RNAi was working. Knockdown (KD) of drp-1 in wild type worms leads to occurrence of blebs that are still connected by thin threads of outer mitochondrial membrane. In catp-6 mutants, knockdown of $d r p-1$ does not aggravate mitochondrial morphology. No blebs are seen upon drp--1 KD in catp- 6 mutants. RNAi against the fission gene fis- 1 leads to mitochondrial elongation in wild type worms but does not have any impact on mitochondrial morphology or DRP-1 clustering in catp-6. KD of the mitochondrial fusion genes eat-3 and fzo- 1 induces mitochondrial fragmentation in both wild type and catp-6. Scale bars represent $10 \mu \mathrm{m}$.

As shown in Fig. 5.13, mitochondria in wild type worms in which fis-1 expression was knocked down by RNAi are longer and more interconnected than without RNAi treatment supporting a role of fis- 1 in mitochondiral fission in C. elegans. Remarkably, DRP-1 spots at the mitochondria are still detectable after fis-1 RNAi KD indicating that in C. elegans, fis-1 is not required for mediating DRP-1 oligomerization at the mitochondria. Though, as the spots seen in fis-1 KD worms are smaller in size than in wild type, a supportive role of fis-1 for clustering seems plausible. In catp- 6 mutants, mitochondrial morphology is not altered by 
knockdown of fis-1 expression and also the large clusters of DRP-1 in proximity to the nucleus are unaffected by RNAi against fis-1 (Fig. 5.13). The fact that RNAi against the two fission genes fis- 1 and $d r p-1$ has no effect on mitochondrial morphology in catp- 6 deletion mutants suggests that the pathway mediating mitochondrial fission via $d r p-1$ and fis-1 is already inhibited in catp- 6 mutants so that silencing of components of this pathway has no additive effect on mitochondrial morphology in catp-6.

In contrast to the mitochondrial fission genes, silencing of the fusion genes fzo-1 and eat-3 affects mitochondrial morphology in wild type worms and catp-6 mutants in the same way and induces mitochondrial fragmentation in both cases. Interestingly, occurrence of DRP-1 clusters around the nucleus in catp- 6 mutants is manifestly reduced upon silencing of $f z o-1$ and further investigation is needed to determine how fzo-1 knockdown affects DRP-1 localization.

The results of the RNAi experiments indicate that the balance of mitochondrial dynamics is shifted towards increased fusion in catp-6 mutants. As silencing of components of the mitochondrial fission machinery does not aggravate mitochondrial morphology in the mutants, it seems plausible that the effects seen in the mutants are caused by inhibition of the fission machinery. RNAi-mediated KD of components of the mitochondrial fusion machinery reverses mitochondrial morphology in catp- 6 mutants towards fragmentation indicating that an inhibition of the fusion machinery might be able to reverse DRP-1 inactivation. In agreement with this view is the fact that in fzo-1 RNAi treated catp-6 mutant animals discrete TagRFP-DRP-1 puncta can be seen instead of large DRP-1 clusters characteristic for catp-6. Thus, fine tuning the mitochondrial fission-fusion dynamics by decreasing mitochondrial fusion rates might facilitate the restoration of a more wild type-like mitochondrial morphology in catp-6 mutants.

\subsubsection{Changes in mitochondrial morphology in neurons of catp-6 worms}

To determine whether deletion of catp- 6 also affects mitochondrial morphology in other tissues, we were investigating mitochondrial morphology and TagRFP-DRP-1 distribution in motoneurons of the ventral nerve cord (VNC). Similar to what we had seen in muscle cells, perinuclear accumulation of mitochondria was seen in neurons of catp-6 mutants (Fig. 5.14). Furthermore, TagRFP-DRP-1 puncta in the cell bodies are bigger and increased in number when compared to wild type neurons. Mitochondria in neuronal cell bodies of wild type worms form tubular or ring-like structures around the nucleus while in catp- 6 mutants condensed mitochondrial structures on either side of the nucleus are predominating. Also in the 
neurites, which are projecting underneath the cell bodies, mitochondrial morphology is affected by loss of CATP-6 function. In wild type worms, mitochondria in the neurites are mostly seen as elongated tubular structures. In contrast, mitochondria in the neurites of catp- 6 mutants are often seen as punctate structures indicating mitochondrial fragmentation. But besides these fragmented mitochondria also some elongated and very thin tubular mitochondria can be found resembling the long and thin mitochondria that we had seen in BWM cells. This suggests that also in neurons, deletion of catp- 6 leads to severe changes in mitochondrial morphology. The fact that mitochondrial morphology in neurites is differently affected by DRP-1 loss of function than in neuronal cell bodies and muscle cells might be due to special requirements on mitochondrial motility in the processes. Several features like perinuclear aggregation of mitochondria, increased DRP-1 clusters in proximity to the nucleus and occurrence of long and thin mitochondria are shared between muscle cells and neurons of catp- 6 deletion mutants. Nevertheless, some tissue-specific phenotypes can also be observed - mitochondrial fragmentation as seen in the neuronal processes was not apparent in muscle cells and the network formation that we had observed in muscle cells was not found in the neurons. These differences likely result from different demands on mitochondrial morphology in these divergent cell types.

Wild type
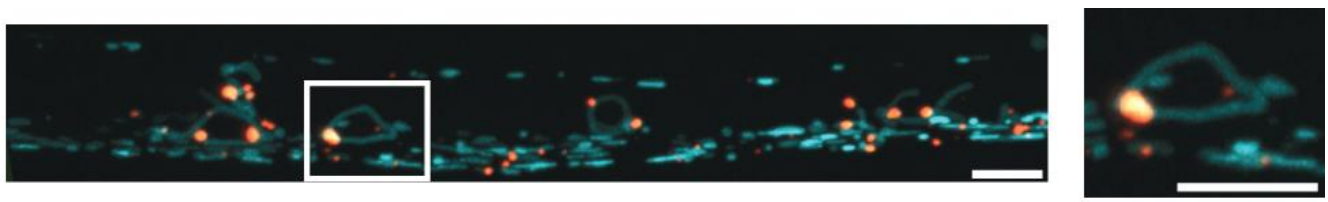

catp-6 (ok3473)
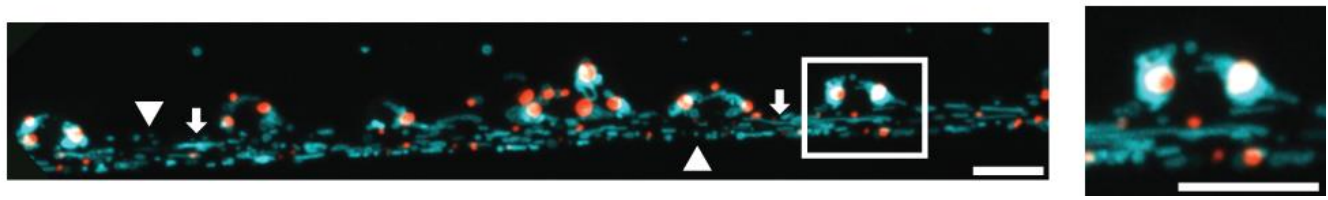

Fig. 5.14: Changes in mitochondrial morphology in neurons of catp-6 mutants.

Changes in mitochondrial morphology were also observed in neurons of the ventral nerve cord of catp-6. As described for muscle cells, mitochondria were labeled with TOM70-CFP and DRP-1 was visualized by a TagRFPTag. While mitochondria in wild type worms form ring-like tubular structures around the nucleus, mitochondria are rather forming clumped aggregates at both sides of the nucleus in catp-6 mutants. Single neurons marked by the white boxes are also shown enlarged in the panels on the right. Changes in mitochondrial morphology are also seen in the neurites of catp- 6 with mitochondria being either fragmented (marked by arrowheads) or longer and thinner than in wild type (marked by arrows). Scale bars represent $5 \mu \mathrm{m}$. 


\subsubsection{Deletion of catp-6 leads to impaired mitochondrial function}

Having observed drastic changes in mitochondrial morphology in catp- 6 deletion mutants we were interested whether these structural changes go along with deficiencies in mitochondrial function. Therefore, we decided to collaborate with the group of Prof. Peter Rehling (Department of Biochemistry II, Georg-August-University Göttingen) to isolate mitochondria from wild type worms and catp-6 mutants and investigate the function of these isolated mitochondria by applying biochemical assays. For mitochondrial isolation, we cultured large quantities of worms and brought the worm pellets to the lab of Prof. Peter Rehling where Dr. Jan Dudek was performing the isolation of the mitochondria as well as the functional assays.

The first step in assessing the functionality of mitochondria in catp- 6 mutants was to determine the mitochondrial membrane potential $\left(\Delta \Psi_{\mathrm{m}}\right)$. The mitochondrial membrane potential is build up as a consequence of proton pumping from the mitochondrial matrix to the intermembrane space by the respiratory chain (see Fig. 2.4). Thereby, a pH and electrical gradient is established across the inner mitochondrial membrane.
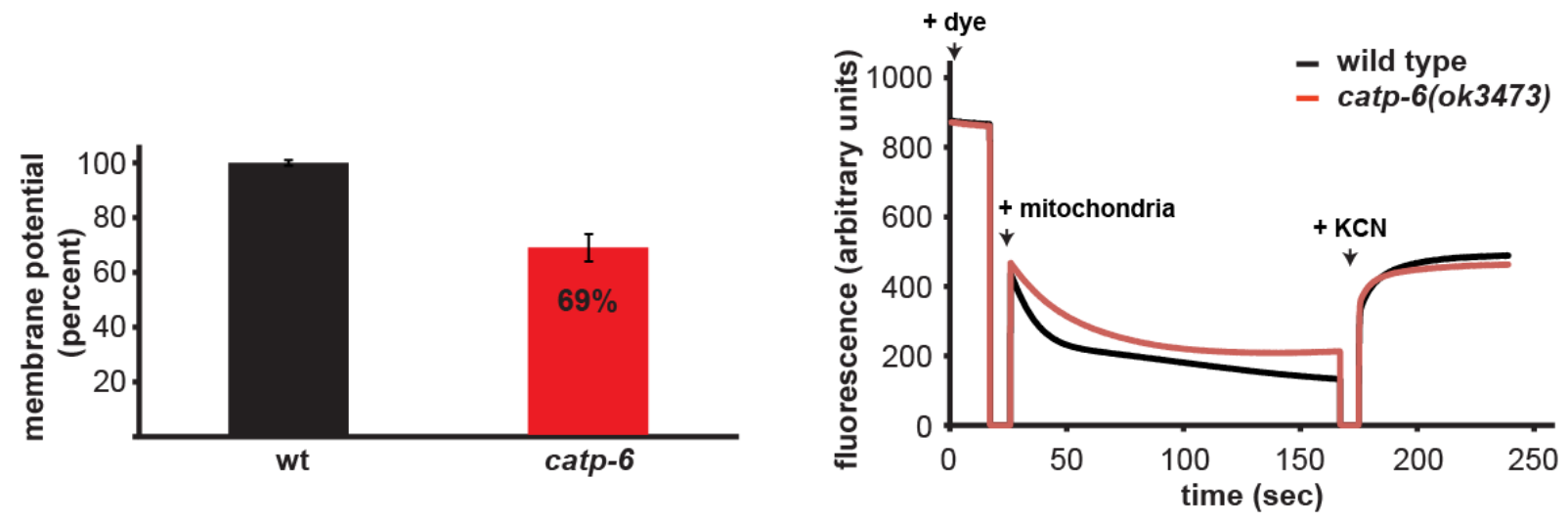

Fig. 5.15: Mitochondrial membrane potential is reduced in catp-6 mutants.

Mitochondrial membrane potential was assessed by measuring fluorescence of DiSC3 which is quenched by the mitochondrial membrane potential. In the first seconds, no fluorescence is detected as only buffer is measured. Addition of the dye then leads to rapid increase of the signal to maximum fluorescence. The fluorescence decreases upon addition of mitochondria due to quenching of the fluorescence. As malate and succinate are added to the reaction, the mitochondrial membrane potential builds up during the measurement and leads to a further decrease in fluorescence. When a stable fluorescence is reached, KCN is added to the solution leading to a specific inhibition of complex IV and therefore an increase in fluorescence. The membrane potential, as determined by the difference in quenching between active and KCN-inhibited mitochondria, is reduced in mitochondria of catp- 6 mutants. Three independent measurements each were performed for wild type and catp- 6 mitochondria and $100 \mu \mathrm{g}$ of mitochondria were used per experiment. Images were kindly provided by Dr. Jan Dudek. 
$\Delta \Psi \mathrm{m}$ can be assessed by the use of lipophilic cationic dyes which easily penetrate cellular membranes and are attracted by the potential across the mitochondrial inner membrane. At the concentration used in this experiment, the $\Delta \Psi_{\mathrm{m}}$-sensitive dye DiSC3(5) is quenched by $\Delta \Psi \mathrm{m}$ leading to a decreased fluorescence with increasing $\Delta \Psi_{\mathrm{m}}$. The results of the $\Delta \Psi_{\mathrm{m}}$ measurements are illustrated In Fig. 5.15. $\Delta \Psi \mathrm{m}$ was calculated as the difference between mitochondrial quenching of active and $\mathrm{KCN}$-intoxicated mitochondria. As shown in Fig. 5.15, $\Delta \Psi_{\mathrm{m}}$ in mitochondria of catp-6 mutants is reduced to $69 \%$ when compared to wild type. This shows that $\Delta \Psi_{\mathrm{m}}$ is severely affected in deletion mutants of catp- 6 which is indicative of compromised mitochondrial function.

Therefore, we decided to investigate whether the mitochondrial respiratory chain is affected by loss of function of CATP-6. The respiratory chain is organized in supercomplexes which are well studied in yeast and mammals. Complex IV assembles with complex III and complex I interacts with complex III as well as several copies of complex IV. The complexes can be separated on BN-PAGE (Blue Native gel electrophoresis) and the activity of respiratory chain complexes can be assessed by in-gel-activity-stains. For staining of complex IV, reduced cytochrome c (cyt c) was added in the gel. Oxidation of cyt c by complex IV is supported by the diaminobenzidine specifically reducing cyt c. Thereby generated oxidized diaminobenzidine falls out as a brown precipitate, thus staining for complex IV activity.

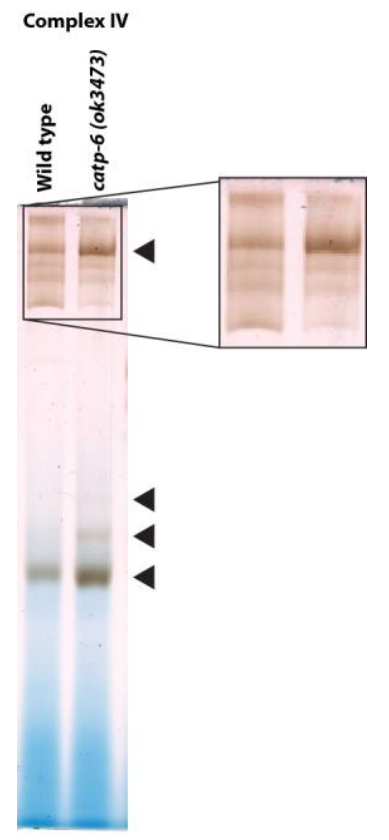

\begin{abstract}
Fig. 5.16: Composition of complex IV of the respiratory chain is changed in catp-6 mutants.

Mitochondrial respiratory chain complexes of solubilized mitochondrial membranes were separated by BN-PAGE (Blue Native Polyacrylamide gel electrophoresis). To stain for complex IV, reduced cytochrome c (cyt c) was added. Oxidation of cyt $c$ by complex IV leads to precipitation of oxidized diaminobenzidine (brown). The staining reveals that some assembly forms of complex IV have taken over a prominent activity in catp-6 (indicated by black arrowheads) while other components show a reduced activity when compared to wild type. The image was kindly provided by Dr. Jan Dudek.
\end{abstract}

The gel picture is depicted in Fig. 5.16 and shows that the composition of complex IV is changed in deletion mutants of catp-6. Higher molecular weight supercomplexes are destabilized in the mutants resulting in an increase in lower molecular weight supercomplexes which is an indication for a dramatic remodeling of the respiratory chain. Further analysis is needed 
to determine whether the components, that show a reduced diaminobenzidine staining, are less active than in wild type or whether the respective protein levels are reduced. As few studies have been published on the composition of respiratory chain complexes in C. elegans and as only a limited number of antibodies for the $C$. elegans respiratory chain complexes are available so far, additional investigations will be needed to elucidate the identity of the different bands that are stained in the gel.

To gain further insight into the respiratory activity of mitochondria in catp- 6 mutants, we decided to determine oxygen consumption of catp-6 mutant worms. Electrons that are transferred through the different complexes of the respiratory chain are transferred to molecular oxygen in complex IV of the respiratory chain. Therefore, the consumption of molecular oxygen reflects the activity of the respiratory chain. Oxygen consumption measurements were performed by me with help of Dr. Jan Dudek using micro-scale oxygraphy for determining the oxygen consumption rate (OCR) and showed that oxygen consumption is increased in catp- 6 mutants (Fig. 5.17).

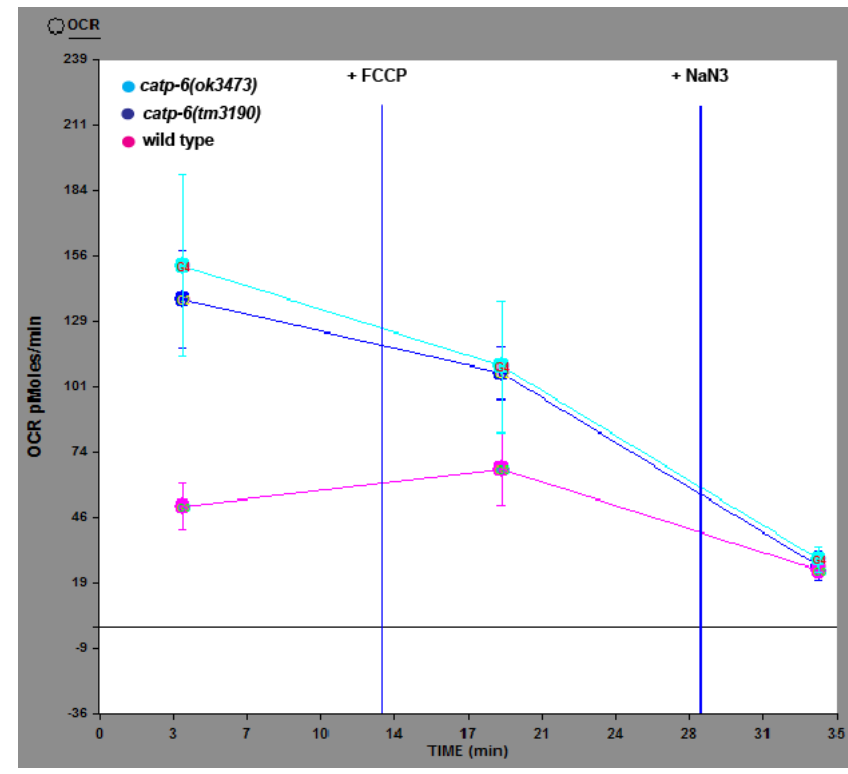

Fig. 5.17: Increased oxygen consumption in catp-6 mutants.

Oxygen consumption of whole worms was measured using a Seahorse 96-well-plate analyzer. Oxygen consumption was first assessed at basal levels, then after addition of the mitochondrial uncoupler FCCP and finally after blocking complex IV by addition of sodium azide $\left(\mathrm{NaN}_{3}\right)$. Oxygen consumption rates in two alleles of catp- 6 were significantly increased at basal levels and in contrast to wild type worms decreased upon addition of FCCP.

One possible explanation for the increased oxygen consumption could be that catp- 6 mutants try to compensate for respiratory deficiencies for example by upregulating mitochondrial mass or by remodeling the respiratory chain. Alternatively, increased oxygen consumption could be caused by less efficient electron transfer at the respiratory chain and consequently increased ROS generation. Therefore, further experiments need to be carried out in order to determine whether mitochondrial mass is changed in the mutants. In this context we will also analyze oxygen consumption in isolated mitochondria as it allows us to normalize oxygen consumption to mitochondrial mass and elucidate whether mitochondrial respiration rates are really increased in catp- 6 mutants or whether this is due to an increased mitochondrial mass. 


\subsubsection{The mitochondrial stress response is induced in catp-6 mutants}

As we could show that both mitochondrial function and morphology, were clearly impaired in catp- 6 deletion mutants, we were wondering whether these deficiencies would also cause an increased induction of the mitochondrial stress response. The mitochondrial stress response is induced by an increased load of misfolded proteins in the mitochondria (Zhao et al., 2002)[reviewed in (Broadley and Hartl, 2008)] similarly to other cellular unfolded protein responses in the cytosol (UPR ${ }^{\text {cyt }}$ ) and in the endoplasmic reticulum (UPR ${ }^{\mathrm{ER}}$ ). As defects in several components of the respiratory chain can lead to an increased production of reactive oxygen species (ROS) (Chen et al., 2003b; Kondo et al., 2005; Kudin et al., 2004; SenooMatsuda et al., 2001; St-Pierre et al., 2002), increased oxidative damage to mitochondrial proteins could be a consequence of compromised mitochondrial function in catp-6. Oxidative damage to the proteins could lead to the accumulation of misfolded proteins in the mitochondria and thus induce the mitochondrial stress response. In C. elegans, the mitochondrial stress response results in increased expression of the mitochondrial chaperones $h s p-6$ (C. elegans ortholog of mammalian Hsp70) and $h s p-60$ (C. elegans ortholog of mammalian Hsp60) (Yoneda et al., 2004). Therefore, to assay mitochondrial unfolded protein stress, we used a reporter driving GFP expression under the control of the $h s p-6$ promoter which was previously described (Yoneda et al., 2004). Interestingly, a strong GFP signal was observed in the catp- 6 mutants under normal conditions at which wild type worms exhibit only a very faint GFP signal in the tail region (Fig. 5.18A).

Induction of the mitochondrial stress response in wild type worms by treatment with the ROS generator paraquat, which causes increased oxidative stress, leads to GFP expression similar to that of catp- 6 mutants that were grown in the absence of paraquat. Further treatment of catp-6 mutants with paraquat increased expression of the hsp-6::gfp reporter construct even further so that the signal is three-fold higher than that of the paraquat-treated wild type worms. Western Blot analysis of GFP expression confirmed the results obtained by fluorescence microscopy (Fig. 5.18B). This suggests that the mitochondrial stress response is intrinsically active in catp- 6 mutants and can be even further activated under conditions of increased oxidative stress. 


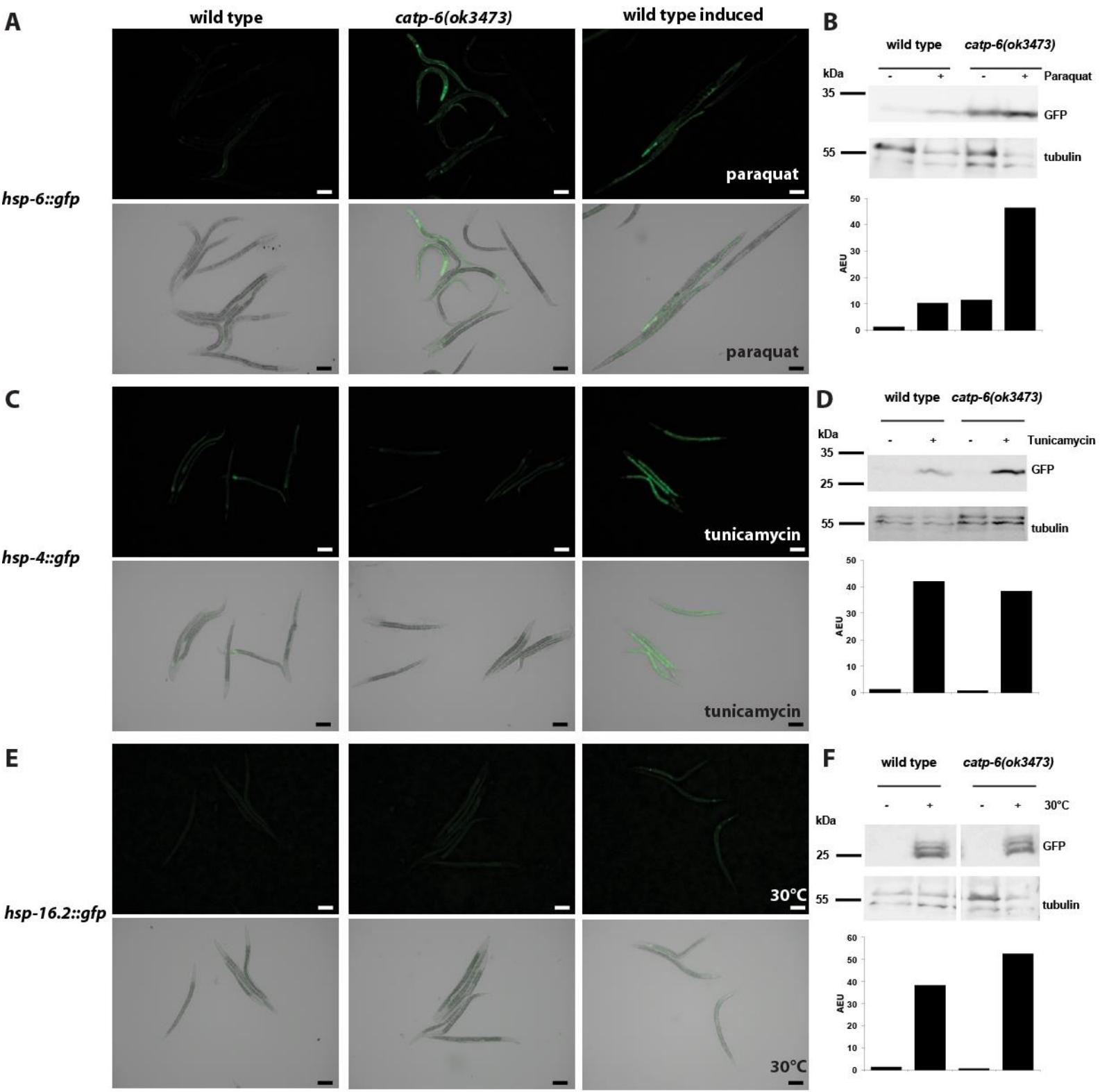

Fig. 5.18: Mitochondrial stress response is specifically activated in deletion mutants of catp-6.

(A-F) Induction of cellular stress responses was assessed using GFP reporter constructs. GFP expression was determined by fluorescence microscopy (A, C, E) and Western Blot (B, D, F). GFP fluorescence is shown in the top panels of $(A, C, E)$ while in the lower panels GFP and bright-field pictures are overlaid. Scale bars represent $100 \mu \mathrm{m}$. Western Blots were quantified by calculating the GFP/tubulin ratio and then normalizing the values for all strains and conditions to the uninduced wild type GFP/tubulin ratio. Induction of the stress responses was done by exposing worms to paraquat, tunicamycin or by exposure to elevated temperature. (A-B) Induction of the mitochondrial UPR was assessed by determining GFP expression from the reporter construct $h s p-6:: g f p$. (C-D) Induction of the endoplasmic reticulum UPR was assessed by determining GFP expression from the reporter construct $h s p-4:: g f p$. (E-F) Induction of the cytosolic UPR was assessed by determining GFP expression from the reporter construct hsp-16.2::gfp. catp-6 mutants show an intrinsic activation of the mitochondrial UPR while ER and cytosolic stress responses are normal. 
In order to exclude that the observed induction of the mitochondrial stress response in catp- 6 mutants is a rather unspecific reaction due to a more generalized morbidity of the mutants, we also examined other cellular stress responses like the UPR ${ }^{\text {cyt }}$ and UPR ${ }^{\mathrm{ER}}$. hsp-16.2::gfp was used as a reporter for the cytosolic heat shock response and $h s p-4:: g f p$ as reporter for the UPR ${ }^{\mathrm{ER}}$ (Calfon et al., 2002; Link et al., 1999). Induction conditions in these cases were heat shock $\left(6 \mathrm{~h}\right.$ at $\left.30^{\circ} \mathrm{C}\right)$ for the cytosolic UPR and treatment with tunicamycin for the $U_{P R}{ }^{E R}$. Both fluorescence microscopy and Western Blot analysis did not show an intrinsic activation of these stress response pathways in the catp- 6 mutants and GFP expression from the reporter constructs upon induction of the stress responses was the same in catp- 6 mutants than in wild type worms (Fig. 5.18C-F). This clearly indicates that the mitochondrial stress response is specifically induced in the mutants probably due to increased oxidative stress.

A closer look at the expression of the $h s p-6:: g f p$ reporter revealed that a number of tissues show strong fluorescence and that the expression pattern differs between individual worms (Fig. 5.19). Among the tissues that seem to be most affected are the pharynx, muscle cells, as well as structures in the vulva region, uterus and gonads. Interestingly, several of these tissues (Vulva, muscle cells, pharynx) were also among the tissues that we had identified in the expression pattern analysis of catp-6. Furthermore, the tissues that are showing a strong induction of the mitochondrial stress response also coincide with the phenotypes of catp- 6 mutants like impaired movement, egg laying defects and reduced brood size.
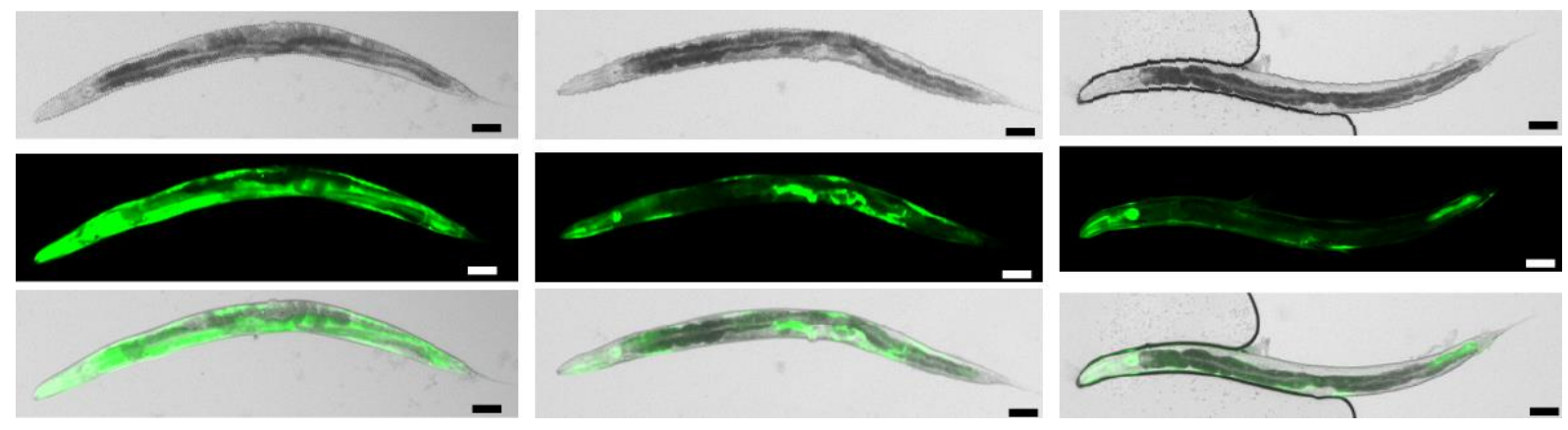

Fig. 5.19: Several tissues of catp-6 mutants show a strong induction of the mitochondrial stress response.

Induction of the mitochondrial stress response in catp-6 mutants was visualized using the $h s p-6:: g f p$ reporter. Among the tissues that are showing a strong GFP signal are the pharynx, muscle cells, the vulva region as well as structures in the gonads. The pattern of induction of the mitochondrial stress response varies between individual worms. Scale bars represent $50 \mu \mathrm{m}$. 


\subsubsection{6. catp-6 mutants exhibit increased sensitivity to oxidative stress and a reduced life span}

We were hypothesizing that activation of the mitochondrial stress response in catp- 6 mutants was due to increased oxidative stress resulting from defects in the respiratory chain. If the levels of reactive oxygen species (ROS) were increased in the deletion mutants, one would expect the worms to show increased sensitivity to treatment with substances that are leading to increased oxidative stress. Therefore, we decided to assess survival upon paraquat treatment. Paraquat induces massive superoxide production by redox-cycling. Paraquat forms a cation radical upon one-electron reduction and electron transfer from this radical to molecular oxygen leads to generation of superoxide and at the same time to the regeneration of the original paraquat molecule which can then start a new redox-cycle (Bus and Gibson, 1984). Paraquat sensitivity was assessed by growing worms on NGM plates containing $150 \mathrm{mM}$ paraquat starting from L4 stage and assessing survival after two days of paraquat treatment. While all of the wild type worms were surviving this treatment, both catp- 6 deletion mutants showed a strongly reduced survival rate of about $60 \%$ of wild type (Fig. 5.20A). This indicates that the deletion mutants are about twice more sensitive to oxidative stress probably due to increased ROS levels in consequence of defects in the respiratory chain.

A

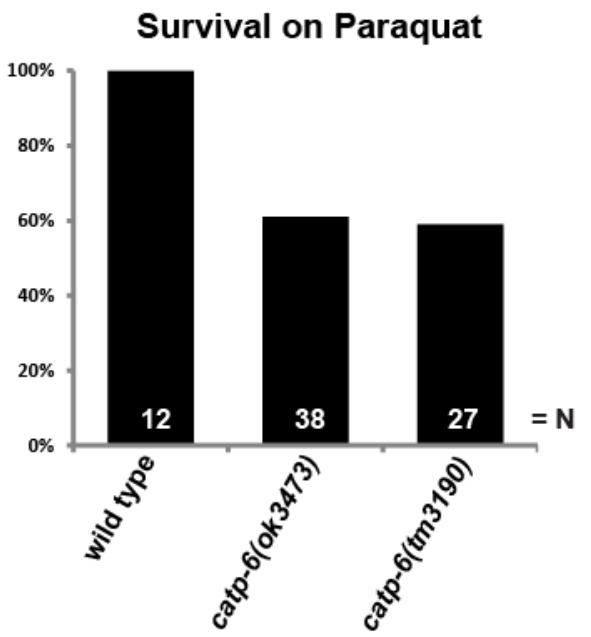

B

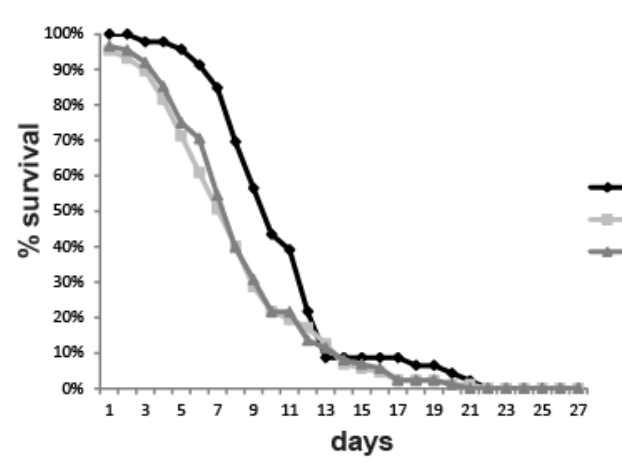

Fig. 5.20: catp-6 mutants show increased sensitivity to paraquat and a reduced life span.

(A) catp-6 deletion mutants show reduced survival after two days of treatment with $150 \mathrm{mM}$ paraquat. (B) Life span is reduced in two alleles of catp-6. To avoid death of catp-6 mutants due to their Egl phenotype, life span was assessed in a glp-4(bn2) background in which gonad formation can be prevented by shifting the worms to the non-permissive temperature between L2 larval stage and late L4 stage. Two independent lines for each allele were analyzed. 
According to the oxidative damage theory of aging, increased oxidative stress accelerates the aging process and causes a reduction in life span (Harman, 1956)[reviewed in (Beckman and Ames, 1998)]. The fact that catp-6 mutants show an induction of the mitochondrial stress response and increased sensitivity to oxidative stress suggests that oxidative stress levels are increased in the mutants and it was therefore tempting to speculate that life span in these worms could be affected. Hence, we analyzed lifespan in two deletion mutants of catp-6. As catp-6 mutants have a strong Egl phenotype which might lead to premature death because of egg accumulation and hatching of larvae inside the parent, we decided to use a $g / p-4(b n 2)$ background for the assays. Switching glp-4 worms to the non-permissive temperature of $25^{\circ} \mathrm{C}$ during postembryonic development prevents the formation of gonads in the worms. The results of the life span analysis are depicted in Fig. 5.20B and show a marked reduction in life span in both catp- 6 deletion mutants. While the maximum life span was unchanged, mean life span of the mutants ( 8 days for both alleles) was reduced when compared to wild type (10 days). $50 \%$ of wild type worms were dead after 10 days of adulthood. In the mutants, this was already the case after 7 or 8 days for catp-6(ok3473) and catp-6(tm3190), respectively. This suggests that deletion of catp- 6 leads to an accelerated aging process possibly by eliciting mitochondrial dysfunction and increased ROS production.

\subsubsection{Increased activation of AMPK and induction of autophagy in catp-6 de- letion mutants}

Having seen the morphological and functional defects of mitochondria in catp- 6 mutants, we were wondering whether this also affects the cellular energy status. If ATP production is diminished in catp- 6 mutants, this should also lead to an increase of the AMP/ATP ratio in the cell. AMPK (AMP-activated protein kinase) senses an increase in the AMP/ATP ratio. Thus, a rise in the AMP/ATP ratio leads to activation of AMPK via phosphorylation of Thr172 by LKB1 (Liver kinase B1; PAR-4 in C. elegans) (Hawley et al., 1996; Lee et al., 2008; Shaw et al., 2004). Therefore, a phosphorylation specific antibody can be used as indirect readout of the energy status of the cell. Western blot analysis of worm lysates showed that the levels of phosphorylated AMPK are augmented in catp-6 mutants. While only a slight increase was observed for the tm3190 allele, the levels of p-AMPK were doubled in catp-6(ok3473) when compared to wild type (Fig. 5.21). 
A

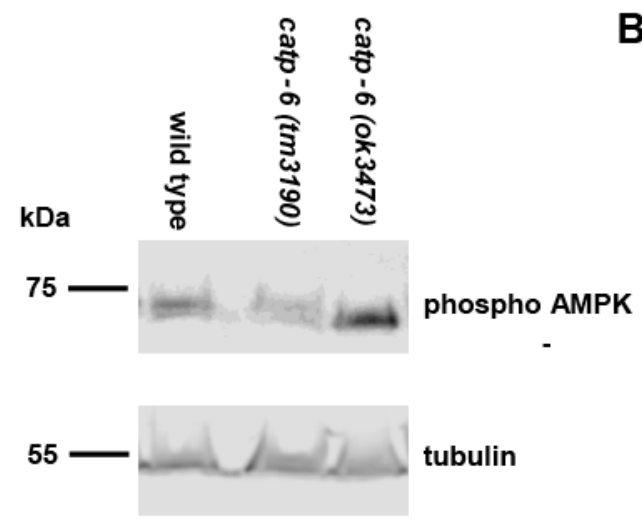

B

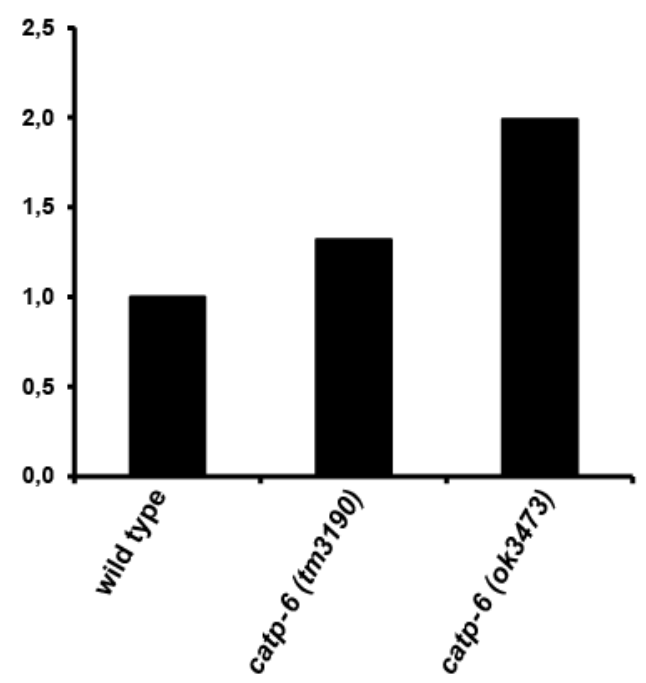

Fig. 5.21: Increased phosphorylation of AMPK in catp-6 mutants.

(A) Western Blot of worm lysates using an antibody against phosphorylated AMPK shows an increased signal for catp-6 mutants. (B) For quantification, a total of four samples from two blots were analyzed. The ratio of p-AMPK and tubulin signal was determined for each lane and the mean of the four samples was calculated. These mean ratios were then normalized to the wild type value to determine AEUs. Phosphorylation is slightly increased in catp-6(tm3190) and a more pronounced increase was seen in the ok3473 allele.

Due to the lack of an antibody against unphosphorylated AMPK we cannot exclude that the levels of total AMPK are affected by catp- 6 loss-of-function but the increase in phosphorylated AMPK hints at a reduction of cellular ATP levels. This would be in line with the functional and morphological defects that were seen in the mutants. Nevertheless, further investigation of the overall AMPK levels are needed to confirm this hypothesis.

Phosphorylation of AMPK has an activating role for cellular catabolic processes while it leads to inhibition of anabolic pathways [reviewed in (Mihaylova and Shaw, 2011)]. One of the effects of AMPK activation is induction of autophagy. This is mediated by inhibition of the mTOR-Raptor complex (mTORC1) which acts as inhibitor of autophagy. Recently, also a more direct influence of AMPK on autophagy has been reported as it was shown that AMKP can activate ULK1 (Unc-51-like kinase 1) which leads to induction of mitophagy (Egan et al., 2011a; Kim et al., 2011). Given the increased phospho-AMPK levels in the catp-6 mutants, we decided to investigate whether increased autophagy can be observed in catp- 6 . We used a previously described reporter strain expressing the GFP-tagged LGG-1 under the control of the endogenous lgg-1 promoter (Kang et al., 2007; Melendez et al., 2003). LGG-1 is the C. elegans ortholog of yeast ATG8 (autophagy related protein 8) and mammalian LC3 (Microtubule-associated protein 1 light chain 3 alpha). As these proteins are conjugated to PE (phosphatidyl ethanolamine) in the autophagosomal membrane, they are commonly used as marker for autophagosomal structures. 


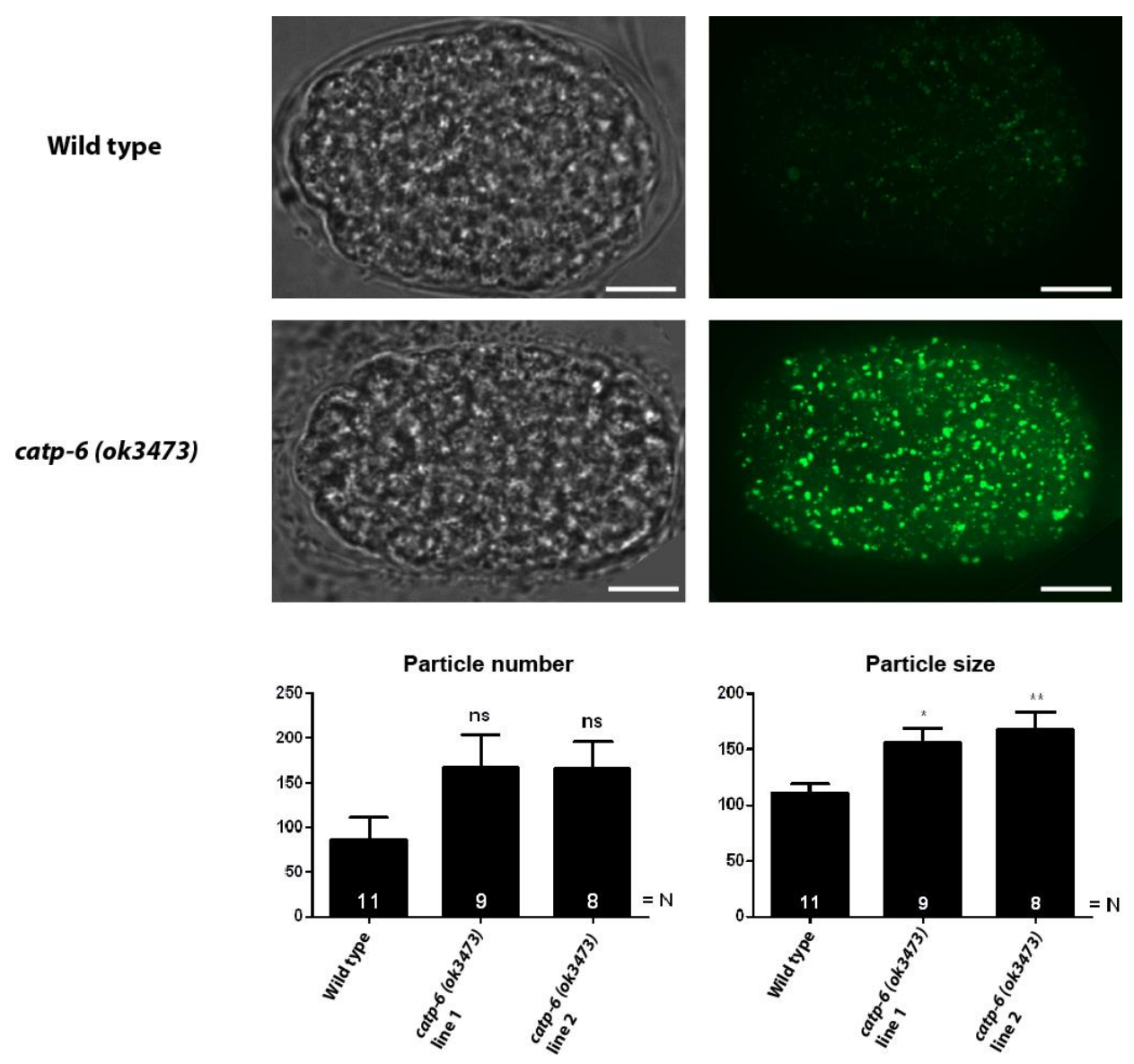

Fig. 5.22: Increased induction of autophagy in eggs of catp-6 mutants.

Eggs of the 150-300 cell stage were analyzed for the number and size of autophagic foci using a reporter strain expressing GFP-LGG-1. Scale bars represent $10 \mu \mathrm{m}$. Both number and size of the foci were found to be increased in catp-6 mutants Two independent lines of catp-6 expressing GFP-LGG-1 were analyzed. Error bars correspond to the standard error of the mean (SEM) and significance values of One-way ANOVA with Dunnett's multiple comparison analysis are indicated: ${ }^{*} \mathrm{P}<0.01,{ }^{*} \mathrm{P}<0.05$, ns $\mathrm{P}>0.05$.

When examining eggs that are expressing the GFP-LGG-1 fusion protein, we observed a marked increase in the number and size of GFP-LGG-1 puncta in eggs of catp- 6 mutants as shown in Fig. 5.22. The increase in autophagic foci could either result from increased induction of autophagy or from decreased clearance. Increased autophagy induction would be in line with the increased levels of phosphorylated AMPK but further experiments need to be done in order to be able to distinguish between these two scenarios. When investigating autophagy in catp- 6 mutants, the increase in autophagic foci was most prominent in eggs of the 150-300 cell stage. We also tried to investigate autophagy in larval stages but there the results were more ambiguous. While in some of the experiments we saw increased number of autophagic foci in the seam cells, we were not able to reproduce these results in all experiments. The reason for this ambiguity might be that we could not control for all parameters that influence autophagy and further investigations are needed to determine which factors are responsible for the divergence between experiments. Therefore, at the moment we can- 
not take a firm stand on whether autophagy is up-regulated in catp- 6 mutants at later developmental stages or in adult worms. It is possible that chronic stimulation of autophagy might lead to compensatory mechanisms that inhibit autophagy or prevent its excessive degradation. Furthermore, it could be that the worms that reach these stages and that have not died during embryonic or early postembryonic development have developed some compensatory mechanisms and that the worms showing more severe phenotypes die before reaching these stages. In accordance with this, we found severe defects in some of the eggs in catp- 6 (Fig. 5.23). These eggs have abnormal appearance in both bright-field images as well as in GFP-LGG-1 fluorescence pictures. Some of the eggs show an abnormal shape and are smaller and more round than for wild type worms. In other eggs, amorphous structures are visible. Moreover, some abnormal GFP-LGG-1 positive structures are found in these eggs which we have never observed in wild type eggs. Given the severe morphological defects of these eggs it seems very likely that those eggs are dead or about to die. This sheds new light on the brood size data as the decreased number of progeny for catp- 6 could at least in part be due to embryonic lethality. Therefore, it will be very interesting to study embryonic development and to check for embryonic lethality.
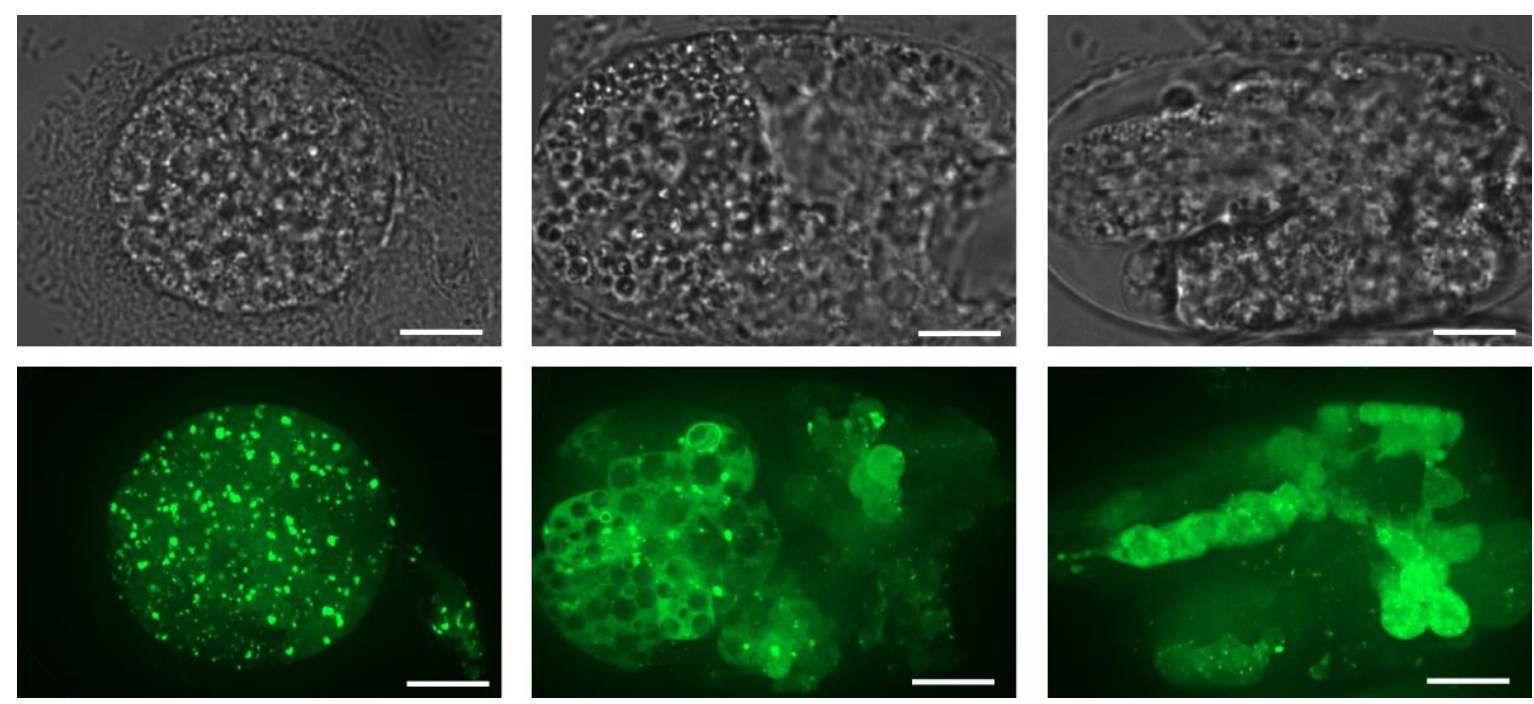

Fig. 5.23: Deletion of catp-6 causes egg defects.

Morphological abnormalities were seen in eggs of catp- 6 deletion mutants. Bright-filed images are shown in the top panels and the lower panels depict fluorescence of GFP-LGG-1. The egg in (A) is smaller and more roundish than eggs of wild type worms which have an ovoid shape. Amorphous structures are seen in the bright-field pictures of $(B)$ and $(C)$ and several anomalous structures are seen in the fluorescence pictures. Scale bars represent $10 \mu \mathrm{m}$. 


\subsubsection{No excessive apoptosis in the germline of catp-6}

As a last aspect of the characterization of catp-6 mutants, we studied germline apoptosis. Increased apoptosis in the germline could be one reason for the reduced brood size in catp- 6 mutants.
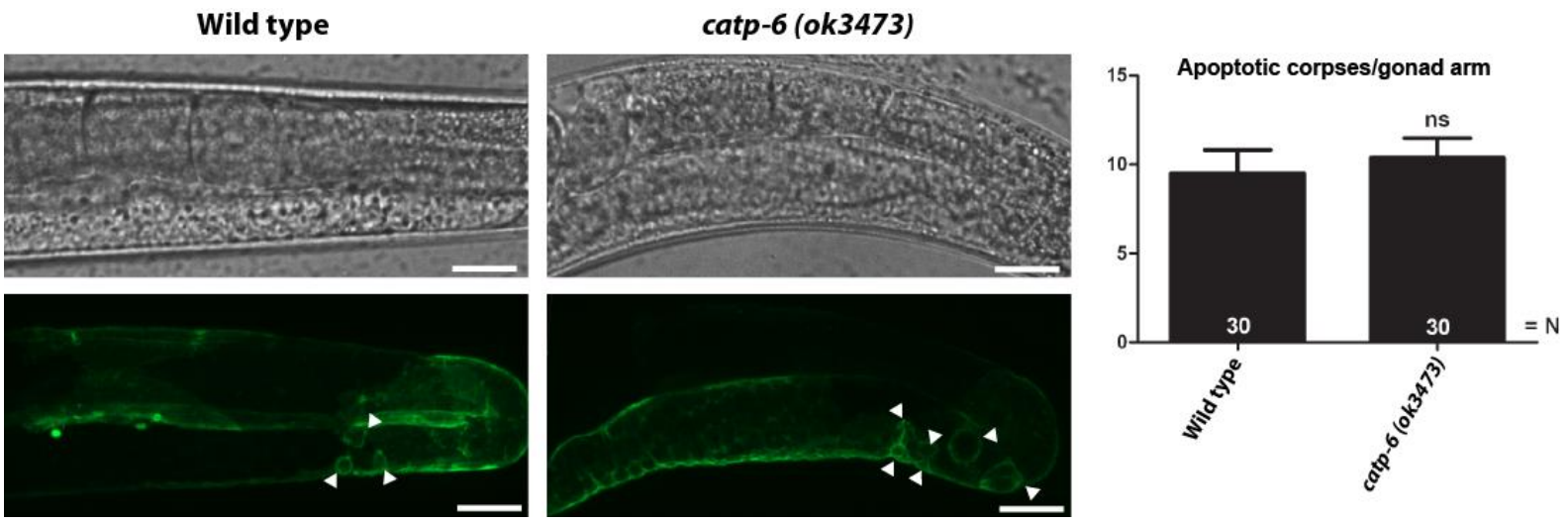

Fig. 5.24: No increase in germline apoptosis in catp-6 mutants.

Apoptotic corpses in the germline of young adult worms were visualized by expression of plim-7::ced-1-gfp. Bright-field images are shown in the top and GFP fluorescence is displayed in the lower panels. Apoptotic corpses are indicated by white arrowheads. Scale bars represent $20 \mu \mathrm{m}$. The overall number of corpses per gonad arm is not changed in catp- 6 mutants.

Apoptosis in the $C$. elegans germline occurs as an intrinsic feature of oogenesis independently of the BH3-only protein EGL-1 which is involved in the regulation of somatic apoptosis in C. elegans (Gartner et al., 2008). The key components of the apoptotic machinery are the same for the germline as for somatic cells. For visualization of apoptotic corpses in the germline, a previously described reporter strain expressing plim-7::ced-1-gfp (Schumacher et al., 2005) was used. Apoptotic cells in the germline are engulfed by the somatic sheath cells. CED-1 is a protein that is expressed at the membrane of the sheath cells and is clustering when the sheath cells are engulfing apoptotic corpses (Zhou et al., 2001). As the lim-7 promoter drives expression of the translational fusion protein CED-1-GFP in the somatic gonad, apoptotic corpses are marked by a ring of fluorescence surrounding the corpses (Schwartz, 2007). The number of apoptotic corpses in the germline was not changed in catp- 6 mutants. This suggests that regulation of apoptosis in the germline is not affected by deletion of catp- 6 and that the reduced brood size cannot be explained by massive dying of germ cells but is rather caused by the Egl and pVul phenotypes or by increased embryonic lethality. 


\subsection{Discussion}

\subsubsection{Multiple factors contribute to the reduced brood size of catp-6 mutants}

The brood size of catp- 6 mutants was found to be severely reduced when compared to wild type worms and this decreased fecundity was accompanied by egg laying (Egl) defects and a protruding vulva phenotype (pVul) (Fig. 5.2 and Table 5.1). C. elegans fertility depends on the cooperative function of several tissues. Oocytes are produced in the gonad where they are fertilized by passage through the spermatheca (Hirsh et al., 1976). Fertilized eggs then enter the uterus where they undergo the first rounds of cell division approximately to the 30 cell stage of embryonic development (Hirsh et al., 1976). Through a concerted action of uterine and vulval muscles, eggs are then expelled through the vulva opening to the outside. During its reproductive period, a C. elegans hermaphrodite typically produces about 300 progeny (Hirsh et al., 1976). The number of progeny is limited by the number of sperm that each hermaphrodite produces during early stages of sexual development (Hirsh et al., 1976). Decreased brood size could therefore either be caused by a low number of sperm or by a massive loss of oocyte forming germ cells. This would, consequently, lead to a decreased total number of fertilized oocytes. As no increase in the number of apoptotic corpses was seen in the germline of catp- 6 mutants, it is unlikely that loss of germ cells accounts for the reduced number of progeny of catp- 6 mutant hermaphrodites. More likely as a cause of the reduced brood size is the egg laying defect in combination with the pVul phenotype of catp- 6 mutants. Furthermore, the accumulation of eggs in the uterus argues for a normal egg production and problems with egg laying. Egg laying relies on contraction of vulval and uterine musculature which requires innervation by HSN and VC motoneurons and a proper vulvauterine connection. In catp-6 hermaphrodites, a severe egg laying defect causes fertilized eggs to be retained within the uterus. In cases of a steric blockade of the vulval opening as a result of the pVul, eggs cannot be laid at all. Therefore, the progeny hatches inside the parent, eventually killing it. Since the capacity of the uterus is limited, this severely restricts the number of progeny that can be produced.

The fact that a protruding vulva is seen in some of the catp- 6 mutants hints at defects in the vulva-uterine connection, which is partially composed by the utse cell (uterine seam cell). The utse cell connects the vulval epidermis to the uterus and is also important for attachment of the uterus to the lateral epidermis. Therefore, the utse cell structurally prevents eversion of the vulva (Newman et al., 1996). Formation of the connection between vulval and uterine lumen requires coordinated specialization of the two tissues (Fig. 5.25). The first step in this process is fate determination of the anchor cell (AC) which depends on LIN-12 (C. elegans 
Notch homolog) and which takes place early in uterine development (Kimble, 1981; Seydoux and Greenwald, 1989). This process functions according to the lateral inhibition principle and results in one of two initially equivalent cells becoming a ventral uterine (VU) precursor cell while the other is acquiring anchor cell (AC) fate. The anchor cell subsequently induces a number of cells in the epidermis to become vulva precursor cells (VPCs) via LIN-3 (epidermal growth factor)/LET-23 (receptor tyrosine kinase) signaling (Aroian et al., 1990; Hill and Sternberg, 1992; Katz et al., 1995; Kimble, 1981; Sternberg and Horvitz, 1986) and then induces a subset of $\mathrm{VU}$ cells to become $\pi$ cells which will form the uterine part of the vulvauterine connection (Newman et al., 1995) (Fig. 5.25a). Induction of the $\pi$ cells involves LIN-12 signaling (Newman et al., 1995).

(a)

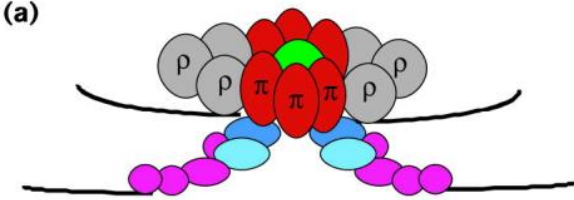

(b)

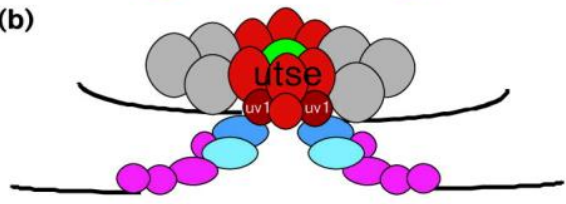

(c)

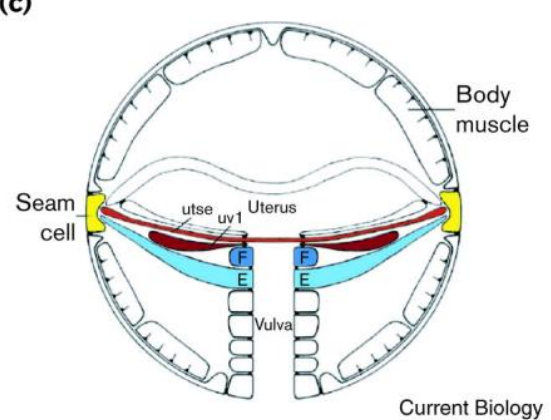

Fig. 5.25: Formation of the vulva-uterine connection in C. elegans.

(a) Uterine $\pi$ cells (red) are induced via signaling from the AC (green). (b) Division of the $\pi$ cells gives rise to uv1 cells (ventral daughters) and utse cells which fuse to form a syncytium. (c) Schematic representation of a cross-section of an L4 larva in which formation of the vulva-uterine connection is completed. The utse (red) has fused to form a thin layer between vulva and uterus and is also forming connections to the seam cells (yellow). Uv1 cells (dark red) are forming the connection of utse and the dorsal vulva cells, VulF (blue) and VulE (cyan). Reprinted from Current Biology, 10, Newman et al., The Caenorhabditis elegans heterochronic gene lin-29 coordinates the vulval-uterine-epidermal connections, p. 1479-1488, Copyright (2000), with permission from Elsevier. (Newman et al., 2000).

After having induced VPCs and $\pi$ cells, the anchor cell builds a barrier between vulva and uterine cells thus allowing the two tissues to differentiate independent from each other (Newman and Sternberg, 1996). The VPCs then differentiate to form the vulva while $\pi$ cells give rise to two different types of uterine cells that are involved in forming the connection between vulva and uterus - utse and uv1 cells (Newman et al., 1996) (Fig. 5.25b). The utse cells fuse to form a multinucleated syncytium to which the anchor cell finally also fuses (Newman et al., 1996). A thin laminar process is formed by the utse which separates vulva and uterus and which is broken by the first egg being laid (Newman and Sternberg, 1996) (Fig. 5.25c). Besides forming this thin lamina, the utse is also making connections to the epithelial seam cells thus preventing eversion of vulva and uterus (Newman and Sternberg, 
1996). Failure in formation of the utse causes a pVul phenotype as seen in catp- 6 worms indicating that the mutants have problems with either induction or differentiation of the $\pi$ cells. uv1 cells, the second cell type generated by the $\pi$ cells, are connecting dorsal vulval cells and utse by forming adherens junctions to both (Newman et al., 1996). As many steps in the formation of the vulva-uterine connection rely on cell-cell signaling between different tissues and lineages and as the signaling is transient in nature (Huang and Hanna-Rose, 2006), it is important that development of the different tissues is well-synchronized. Huang and Hanna-Rose have described a cog-3 mutant (connection of gonad defective; new gene name pnc-1) which fails to form a vulva-uterine connection because development of the uterus in these worms is delayed relative to vulval development (Huang and Hanna-Rose, 2006). As differentiation of the uv1 cells depends on signaling between vulval cells which express LIN-3 (EGF) and uterine $\pi$ cells expressing the LET-23 EGF receptor, asynchrony in development of the two tissues disturbs this signaling as LIN-3 expression by vulval cells happens at a time when the $\pi$ cells are not ready yet to respond to this signal (Huang and Hanna-Rose, 2006). Formation of the vulva-uterine connection in catp-6 mutants could result from similar effects of asynchronous development. We have found that postembryonic development is strongly delayed in catp-6 (Fig. 5.3) and it is tempting to suggest that not all tissues are affected to the same extent by this delayed development. This may prevent adequate cell-cell signaling and thus disturb the formation of the vulva-uterine connection. Interestingly, we found strong expression of catp- 6 in the vulva indicating that this tissue might be particularly sensitive to deletion of catp-6, which could cause a stronger delay in the development of this tissue when compared to others including the uterus. This would be in line with the hypothesis of asynchronous development interfering with the formation of the vulvauterine connection. It would be interesting to have a closer look at the developmental delay and to check whether vulva and uterus development are shifted in time. If, as in the case of cog-3 mutants, vulval development is proceeding faster than the development of the uterus one might try to rescue the effect by ectopic expression of LIN-3 in the appropriate time window or by expression of a LET-23 gain-of-function as in the case of $\operatorname{cog}$-3 this was sufficient to rescue the uv1 fate defect (Huang and Hanna-Rose, 2006).

Besides the pVul phenotype, we found several defects in the vulva muscles of catp- 6 mutants, which are also likely to contribute to the Egl defect and the reduced brood size. The vulva muscle defects observed were ranging from irregular arrangement of the muscles relative to the vulva and defects in the attachment of the muscle cells to the lack of one or more vulva muscles (Fig. 5.8 and Table 5.2). Similar defects have been described for mutants in the Notch pathway in C. elegans, lin-12 and sel-12 (Eimer et al., 2002). C. elegans vulva muscles belong to the mesodermal $M$ lineage which gives rise to body wall muscle (BWM) 
cells, coelomocytes (CC) and sex myoblast (SM) cells. The sex muscles including both vulva and uterine muscles originate from the sex myoblast (SM) cells, which are at first located in the tail of the worm and during the L2 larval stage migrate towards the site where the vulva will be formed. The vulva muscles in catp- 6 that are not properly attached to the vulva and show irregular arrangement have migrated successfully towards the vulva region and are able to differentiate into muscle cells but probably have defects in cell-cell signaling which would normally ensure correct positioning and alignment of the muscles relative to the vulva. This is required for a proper connection between vulva and vulva muscles and thus for proper egg laying. As described above, asynchrony in the development of different tissues - in this case probably the vulva and sex muscles - would be a possible explanation for the defective signaling resulting in defects in muscle morphology. The observation that in around $50 \%$ of catp- 6 worms at least one vulva muscle is missing could be either due to migration defects of the SM cells or could be caused by defects in the SM fate determination. While migration defects with differentiated vulva muscles residing in the tail region of the worm have been found in sel-12 mutants (Eimer et al., 2002), a fate determination defect was seen in lin-12 mutants, where instead of acquiring SM fate, the cells were adopting CC fate resulting in missing vulva muscles and increased numbers of CCs (Greenwald et al., 1983). C. elegans CCs are scavenger cells that continuously endocytose the body cavity fluid. Wild type worms have six CCs and increased numbers of CCs were found in lin-12 null mutants. Therefore, it would be interesting to determine the number of CCs in catp- 6 mutants and look for the presence of vulva muscles expressing the marker hlh-8::gfp in the tail region of the worm as these experiments would allow to distinguish between the two scenarios. In either case, defective signaling due to asynchronous development could be disrupting the signaling that induces SM fate or that controls migration of the sex muscles. Interestingly, all the signaling steps that might be affected in catp- 6 mutants ( $\pi$ cell fate determination, SM fate determination, SM migration, control of arrangement of vulva muscles and attachment to the vulva) rely on LIN-12 (Notch) signaling. Thus, another explanation for the defects seen in the mutants would be that loss of CATP-6 function somehow disrupts LIN-12/Notch signaling during the development of the egg laying system. Further investigations are needed in order to determine whether and how the lysosomal P-type ATPase CATP-6 could affect LIN-12 signaling.

A third factor that, in addition to the defects in vulva muscles and the vulva-uterine connection, is likely to contribute to the reduced brood size of catp- 6 mutants is embryonic lethality. We have observed severe morphological defects in embryos of different developmental stages (Fig. 5.23) indicating that loss of CATP-6 function also causes embryonic lethality. We have shown that dying embryos display excessive induction of autophagy. However, during 
larval development and in the adult stage, autophagy is not strongly up-regulated anymore. This suggests that there might be a crisis during early embryonic development in catp- 6 mutant embryos where additional energy has to be generated through autophagy. catp- 6 mutant embryos that are able to overcome this crisis display slow rates of development but are able to mostly reach the reproductive stage. These adult catp- 6 mutant animals exhibit mostly normal rates of autophagy suggesting that they are able to down-regulate excessive autophagy during postembryonic development. However, there seems to be a proportion of embryos that do not manage to generate enough energy for embryonic development through autophagy and subsequently die. This is consistent with the fact that those embryos that show the brightest and largest number of GFP-LGG-1 positive puncta are destined to die. Excessive induction of autophagy has been linked to cell death in $C$. elegans in previous studies (Kang et al., 2007). Therefore, it will be interesting to assess embryonic development in the mutants to determine how many worms are affected by the embryonic lethality.

In conclusion, we have shown that three independent defects contribute to the reduced brood size in catp-6: a defective vulva-uterine connection, defects in vulva muscles as well as embryonic lethality. The defects of the egg laying apparatus could all be explained by either defective Notch signaling or asynchronous development of different tissues and cell lineages preventing normal cell-cell signaling that is needed for the proper formation of these structures.

\subsubsection{Reduced locomotion of catp-6 is based on muscular defects that are al- ready apparent at larval stages}

We observed that catp- 6 mutants have impaired locomotory activity when compared to wild type worms (Fig. 5.4). As the movement defect was already apparent at larval stages, it seems not to be the consequence of a degenerative process. We did not find any abnormalities in structure and arrangement of the myofilaments (Fig. 5.6) but when investigating mitochondrial morphology in body wall muscle (BWM) cells of the mutants, we found severe changes with networks of long, thin and interconnected mitochondria and perinuclear clustering of mitochondria (Fig. 5.8, Fig. 5.11). Overexpression of CATP-6 specifically in muscle cells of the mutants rescued both mitochondrial morphology (Fig. 5.8) and movement (Fig. 5.4). This indicates that the movement defect is caused by a muscular dysfunction due to loss of CATP-6 function rather than by problems with neuronal input to the BWMs. This is in line with the expression pattern of CATP-6, which showed expression of CATP-6 in muscle cells (Fig. 5.5). Whether the changes in mitochondrial morphology in BWMs in catp-6 dele- 
tion mutants are causative for the impaired locomotion of the worms or whether both movement defects and changes mitochondrial dynamics result from a yet undetermined effect of loss of function of CATP-6 needs to be subjected to further investigations. It would be interesting to investigate how shifting mitochondrial dynamics towards increased fission in catp-6 mutants - as it has been achieved by RNAi against the mitochondrial fusion genes fzo- 1 and eat-3 (Fig. 5.12) - affects movement in the mutants. As we have observed a functional impairment of the mitochondria in catp-6 mutants (Fig. 5.15-17) in addition to the morphological changes, it is questionable whether changing the balance of mitochondrial fission/fusion alone without ameliorating the functional deficits, will be sufficient to rescue the movement defects.

\subsubsection{Deletion of catp-6 leads to changes in mitochondrial morphology}

We have found drastic changes in mitochondrial morphology of catp- 6 mutants both in muscle cells as well as in neurons. Mitochondria in the muscles of catp- 6 mutants were much thinner, longer and more interconnected than in wild type and perinuclear clustering of mitochondria was observed (Fig. 5.9). Neurons lacking CATP-6 function display aggregation of mitochondria in the neuronal cell bodies with large clusters of mitochondria observed next to the nucleus (Fig. 5.14). In the neurites, mitochondrial fragmentation but also some long and very thin tubules were observed.

Very recently, a number of studies have reported changes in mitochondrial morphology upon siRNA mediated KD of ATP13A2, the human ortholog of catp-6, in cell culture. Mitochondrial fragmentation has also been reported in cultured neurons (Ramonet et al., 2012), SH-SY5Y cells (Gusdon et al., 2011) and in fibroblasts of KRS patients carrying mutations in the ATP13A2 gene (Grunewald et al., 2012). The mitochondrial fragmentation observed in these models is in contrast to the elongated thin and interconnected mitochondria that we have observed in the muscle cells of catp-6 worms (Fig. 5.9). As we have seen mitochondrial fragmentation in neurons of $C$. elegans catp- 6 mutants (Fig. 5.14), it might depend on the cell type in which way mitochondrial dynamics are changed upon loss of CATP-6/ATP13A2. It is known that mitochondrial dynamics differs between cell types as a consequence of variable metabolic demands [reviewed in (Kuznetsov et al., 2009; Sauvanet et al., 2010)]. Therefore, it seems plausible that the effects of CATP-6 inactivation on mitochondrial morphology also vary between cell types.

Interestingly, mitochondrial elongation, network formation and perinuclear clustering in BWM cells of catp- 6 mutants were accompanied by changes in the localization of the mitochondrial 
fission protein DRP-1 which aggregates in large clusters in close proximity to the nucleus in catp-6 mutants while individual, equally distributed puncta are seen in wild type muscles (Fig. 5.12). DRP-1 in wild type worms is recruited to sites on mitochondria where fission is going to take place and it oligomerizes around the mitochondria before it helps constricting the membranes and dividing the mitochondrion (Labrousse et al., 1999). The sites of DRP-1 oligomerization at the mitochondria are visible as defined puncta in wild type worms. The fact that DRP-1 is forming large aggregates in catp-6 mutants suggests that normal DRP-1 mediated mitochondrial fission is prevented in the mutants as DRP-1 is "trapped" in the aggregates and cannot oligomerize at sites of fission. Inhibition of fission would also be consistent with the very long mitochondrial tubules observed in the mutants. Drp1 activity is regulated by posttranslational modifications and the phosphorylation status of Ser637 in the GED domain (GTPase effector domain) plays a very important role in determining fission activity of the protein. The GED domain of Drp1 is located at the C-terminus and is involved in both intra- and intermolecular interaction with other Drp1 molecules and these interactions are important for stimulation of GTPase activity and oligomerization, respectively (Zhu et al., 2004). Two independent studies have shown that Ser637 in the GED domain is phosphorylated by the cAMP-dependent protein kinase (PKA) resulting in decreased GTPase activity. They also demonstrated that a phosphomimetic Ser637Asp mutation leads to inhibition of mitochondrial fission resulting in elongated mitochondria (Chang and Blackstone, 2007; Cribbs and Strack, 2007). Cribbs and Strack also showed that Ser637 is dephosphorylated in a $\mathrm{Ca}^{2+}$-dependent manner by calcineurin and that this dephosphorylation promotes mitochondrial fission as seen in cells expressing a Ser637Ala mutant Drp1, which cannot be phosphorylated. Cereghetti et al. reported that dephosphorylation of Ser637 by calcineurin enables translocation of Drp1 to the mitochondria and that the anti-fission effect of phosphomimetic Ser637Asp mutation is caused by failure of Drp1 to associate with mitochondria rather than with decreased GTPase activity (Cereghetti et al., 2008). Considering this phosphorylation-dependent activity of Drp1, mitochondrial elongation and aggregation of DRP-1 in the cytosol of catp- 6 mutants could be explained by increased phosphorylation of DRP-1, preventing its translocation to the mitochondria and thus fission. Increased Ser637 phosphorylation could be caused by a rise in cAMP levels and thus increased PKA activity or by reduced activity of calcineurin. This is interesting as it suggests that DRP-1 inactivation and mitochondrial elongation could be protective for the cells. We are currently working on determining the phosphorylation status of TagRFP-DRP-1 in catp- 6 mutants by immunoprecipitation and subsequent mass spectroscopic analysis, in order to check whether the hypothesis of increased Ser637 phosphorylation holds true. Furthermore, we are planning to examine the effects of RNAi against tax-6 (C. elegans calcineurin ortholog) and kin-1 (C. elegans ortholog of PKA) on mitochondrial morphology in catp-6 mutants and wild type 
worms. If increased phosphorylation of DRP-1 Ser637 causes mitochondrial morphology defects and DRP-1 aggregation in the mutants, RNAi against kin-1 should ameliorate mitochondrial morphology in the mutants by preventing phosphorylation while RNAi against tax- 6 should lead to changes in mitochondrial morphology in wild type worms resembling those seen in catp- 6 mutants by preventing dephosphorylation. Similarly, treating catp- 6 mutants with $\mathrm{H}-89$, an inhibitor of PKA, should rescue mitochondrial morphology and DRP-1 localization. In this context it would also be interesting to investigate mitochondrial morphology in worms expressing a tax-6/calcineurin gain-of-function, which should show increased fragmentation, or in deletion mutants of tax-6/calcineurin, which should exhibit mitochondrial elongation as seen in catp- 6 worms. And finally expressing tax-6/calcineurin gain-of-function in catp-6 mutants should rescue mitochondrial morphology if indeed increased Ser637 phosphorylation is the cause for mitochondrial elongation.

To determine how the mitochondrial fission-fusion dynamics are affected in catp-6 mutants, we performed RNAi against components of the mitochondrial dynamics machinery in catp- 6 mutants. We found that silencing of the fission genes $d r p-1$ and fis-1 does not lead to aggravation of mitochondrial morphology in catp-6 (Fig. 5.13). This is in line with our hypothesis that DRP-1 activity is prevented in the mutants by increased DRP-1 phosphorylation, so that loss of the protein does not lead to additional anti-fission effects. Interestingly, silencing of DRP-1 in wild type worms leads to matrix-filled blebs that are connected by very thin tubules of outer mitochondrial membrane indicating that fission of the inner membrane can still occur in these cells. In contrast, these blebs are not seen in catp- 6 mutants indicating that fission of the inner mitochondrial membrane (IMM) is also inhibited in the mutants, which cannot be explained by inactivation of DRP-1 alone. This is very interesting as it has been proposed that a distinct machinery for division of the IMM exists but the components of this machinery have not been found yet (Labrousse et al., 1999). RNAi against the mitochondrial fusion genes fzo-1 and eat-3 induced mitochondrial fragmentation in both wild type worms and catp-6 mutants. From this data it is hard to judge whether increased mitochondrial fusion could also contribute to mitochondrial elongation in catp- 6 mutants as loss of the fusion genes is shifting the balance between fission and fusion towards increased fission and thus can counteract mitochondrial elongation caused by either increased fusion or inhibited fission in the mutants. In order to distinguish between the two scenarios that could lead to mitochondrial elongation in catp-6 - increased fusion or inhibited fission - it would be necessary to follow mitochondrial dynamics by live cell imaging.

Whether the changes in mitochondrial morphology in catp- 6 mutants are detrimental to the cell or whether they rather are a compensatory mechanism preventing massive mitochondrial 
degradation and maximizing the ATP production remains to be determined. Parone et al. have shown that inhibiting mitochondrial fission by depletion of Drp1 in HeLa cells causes loss of mtDNA, impaired mitochondrial respiration, reduced ATP levels and increased autophagy (Parone et al., 2008). In contrast, Cribbs and Strack have reported that decreased fission due to phosphorylation of Drp1 at Ser637 leads to protection of cells against treatment with inducers of apoptosis such as staurosporine, a protein kinase inhibitor, as well as against $\mathrm{H}_{2} \mathrm{O}_{2}$ (Cribbs and Strack, 2007). A protective function of mitochondrial elongation has also been shown for conditions of nutrient deprivation (Gomes et al., 2011). Gomes et al. have demonstrated that starvation induces phosphorylation of Drp1 by PKA via increased cAMP levels thus inhibiting mitochondrial fission and preventing mitochondria from being degraded via autophagy which is induced by nutrient depletion. Preventing mitochondrial elongation during starvation by inhibition of PKA or deletion of Opa1 leads to decreased viability of the cells (Gomes et al., 2011). PKA-mediated mitochondrial elongation not only serves as a protective mechanism to prevent massive mitochondrial degradation but also helps to sustain cellular ATP levels by higher number of cristae and thus more efficient oligomerization of the ATP synthase (Gomes et al., 2011). Thus, further investigations are needed to determine whether mitochondrial elongation in catp- 6 mutants also serves to protect the cells against oxidative stress, autophagic degradation and to ensure sufficient ATP supply. For this purpose it might be interesting to analyze the effects of KD of the fusion genes eat-3 or fzo-1 in catp- 6 deletion mutants on mitochondrial function, ATP levels, oxygen consumption but also the sensitivity towards oxidative stress. As we have shown that silencing of these genes prevents mitochondrial elongation in the mutants, these studies would help to determine how elongation influences mitochondrial function.

Perinuclear clustering has been described to occur during mitophagy, a mechanism leading to selective degradation of damaged mitochondria (Okatsu et al., 2010). The two PD-related proteins PINK1 and Parkin play an important role in targeting damaged mitochondria with low membrane potential to autophagic degradation (Geisler et al., 2010; Matsuda et al., 2010; Narendra et al., 2008; Narendra et al., 2010; Vives-Bauza et al., 2010b). Okatsu et al. describe that induction of mitophagy by treating cells with the mitochondrial uncoupler CCCP leads to accumulation of aggregated mitochondria in close proximity to the nucleus. They furthermore show that this perinuclear clustering is dependent on Parkin-mediated ubiquitylation, p62 and microtubule transport and propose that the mechanism leading to juxtanuclear mitochondrial aggregation is analogous to aggresome formation, a mechanism by which the cell sequesters misfolded proteins into large aggregates which accumulate next to the nucleus where they are subsequently degraded by autophagy (Okatsu et al., 2010). The fact that we also see perinuclear clustering in catp- 6 mutants could hint at increased induction of mi- 
tophagy which would be in line with the reduction in membrane potential and increased oxidative stress that we have found in mitochondria of the deletion mutants, as those are known to be triggers for mitophagy. It is possible though, that loss of function of the lysosomal P-type ATPase CATP-6 impairs lysosomal function and thus clearance of mitochondria via mitophagy. In this case, induction of mitophagy in the mutants due to mitochondrial defects would cause mitochondrial aggregates in proximity to the nucleus that cannot be cleared and thus become even more pronounced. If damaged mitochondria are not cleared properly, this could contribute to increased oxidative stress in the mutants as damaged mitochondria are known to produce more ROS. Another condition for which perinuclear clustering of mitochondria has been reported is mitoptosis (programmed mitochondrial death), which is a relatively novel concept of mitochondrial clearance independent from extramitochondrial proteins [reviewed in (Apostolova et al., 2011)]. During this process, mitochondria form clusters in the perinuclear region, so called "mitoptotic bodies" which are surrounded by a membrane and in which the mitochondria are degraded. In catp- 6 mutants, we see some large spots with strong TOM70-CFP staining close to the nucleus, which resemble inclusion bodies (Fig. 5.9). Those could be mitoptotic bodies as the TOM70-CFP signal is not limited to the outer membrane in these structures but rather stains the whole structure indicating that they have lost the normal mitochondrial structure possibly by degradation of the mitochondrial components. It would be interesting to investigate these formations by Electron microscopy to determine the ultra-structural nature of these mitochondrial accumulations in the perinuclear region.

In summary, we have shown that deletion of catp-6 leads to changes in mitochondrial morphology and that mitochondrial elongation in the mutants is possibly induced by PKAmediated inhibition of DRP-1 fission activity. Whether mitochondrial elongation is causing impaired mitochondrial function by hindering the elimination of damaged mitochondria or whether it is rather a compensatory mechanism to maintain cellular ATP production and to protect against massive loss of mitochondrial mass by autophagic degradation will be the subject of future studies.

\subsubsection{Impaired mitochondrial function in catp-6 deletion mutants}

Besides morphological changes we also observed effects on mitochondrial function in catp- 6 mutants. The mitochondrial membrane potential $\left(\Delta \Psi_{\mathrm{m}}\right)$ is strongly reduced and catp- $6 \mathrm{mu}$ tants show higher oxygen consumption rates. Furthermore, we found that the assembly of complex IV of the respiratory chain is changed in catp- 6 mutants. It has to be noted that the functional assays were performed on whole worms (oxygen consumption) or on isolated mitochondria that were also extracted from whole worms. Therefore, the results display overall 
effects of a mixture of mitochondria from different tissues. As we had observed different effects of CATP-6 loss-of-function on mitochondrial morphology in different tissues, i.e. muscle cells and neurons, it is possible that mitochondrial function is also affected in different ways in these cells. It would be desirable to assess mitochondrial function in a tissue-specific way or ideally even in individual cell as this would allow correlations between mitochondrial morphology and function but methods for such analyses are not available up to present.

As $\Delta \Psi_{\mathrm{m}}$ is built up as a consequence of the proton pumping activity of the respiratory chain which is coupled to the electron transfer activity, loss of $\Delta \Psi_{\mathrm{m}}$ in catp- 6 mutants indicates a dysfunction of the electron transfer chain (ETC). Alternatively, differences in the membrane permeability could also disturb the proton gradient. In a recent study, Grünewald et al. have investigated mitochondrial membrane potential in fibroblasts from KRS patients carrying mutations of the human catp-6 ortholog ATP13A2. They demonstrated that loss of ATP13A2 function significantly reduces $\Delta \Psi_{\mathrm{m}}$ analogous to what we see in catp-6 mutants (Grunewald et al., 2012).

Whether the changes in the composition of complex IV, that we have seen using in-gelactivity-staining in blue native poly-acrylamide gel electrophoresis (BN-PAGE), contribute to the reduced $\Delta \Psi_{\mathrm{m}}$ will need to be determined. Complex IV is the cytochrome c (cyt c) oxidase which accepts electrons from cyt $c$ and transfers them to molecular oxygen thus generating $\mathrm{H}_{2} \mathrm{O}$. This process is coupled to transport of protons from the mitochondrial matrix to the intermembrane space. Thus, it is possible that changes in complex IV can lead to decreased proton pumping and thus could contribute to a reduction in membrane potential. Nevertheless, proton pumping also occurs at complexes I and III so that a reduced proton gradient could also result from defects in these processes (compare Fig. 2.4). Furthermore, reduced efficiency in any of the complexes would go along with decreased proton pumping, so that it is difficult to infer form a loss in $\Delta \Psi_{\mathrm{m}}$ in which way ETC function is compromised. Besides a failure in proton pumping, leak of protons through the membrane due to changes in permeability of the membrane could be the cause for a reduction in $\Delta \Psi \mathrm{m}$. Although it needs to be determined what causes the decreased $\Delta \Psi_{\mathrm{m}}$ in catp- 6 mutants, loss of $\Delta \Psi_{\mathrm{m}}$ hints at reduced ability of the mitochondria to produce ATP as the proton gradient is the driving force for ATP production. The fact that we observed increased phosphorylation of AMPK in catp- 6 mutants supports the notion of reduced ATP levels. We are planning to directly assess the ATP content of catp-6 mutants and compare it to that of wild type worms by using commercially available kits that are based on luciferase activity which is dependent on the ATP concentration. 
Astonishingly, we found that oxygen consumption of catp-6 mutant worms is increased when compared to wild type which at first sight seems contradictory to the reduced membrane potential and presumable deficiencies of the respiratory chain. A possible explanation for the increased oxygen consumption rates could be increased mitochondrial mass in the mutants. Increasing mitochondrial mass could either be a compensatory mechanism of the cells to counteract mitochondrial deficiencies and to sustain sufficient ATP supply or it could simply be the consequence of failure to degrade mitochondria. Defects in mitophagy in catp-6 mutants could result from loss of function of the lysosomal P-type ATPase and could even be causative to the mitochondrial dysfunction that we observe in the mutants. Mitophagy is a mechanism for the cell to eliminate dysfunctional mitochondria [reviewed in (Wang and Klionsky, 2011)] which is extremely important given the fact that defective mitochondria are producing higher amounts of ROS thereby causing damage to many cellular components. Therefore, deficient mitophagy might cause on the one hand mitochondrial dysfunction but on the other hand might also affect general fitness of the cell due to increased oxidative stress which would explain the phenotypes that we have seen in catp- 6 mutants. Due to lack of specific markers for mitophagy, we are currently not able to directly assess whether this process is affected in catp- 6 mutants but we will try to get an estimate of mitochondrial mass by quantifying the number of mtDNA copies using real-time PCR, as increased mitochondrial mass would explain why more oxygen is consumed by the mutants. In this respect, we will also determine oxygen consumption in isolated mitochondria of catp- 6 mutants, as this allows us to normalize oxygen consumption rates to mitochondrial mass. If the increased consumption that we have observed for measurement with whole animals cannot be reproduced in the isolated mitochondria this would support the hypothesis that increased mitochondrial mass accounts for the elevated oxygen consumption rates. In a very recent study Gusdon et al. report that siRNA-mediated knockdown of ATP13A2 in SH-SY5Y cells leads to increased oxygen consumption and they show that this goes along with increased mitochondrial mass (Gusdon et al., 2011) which supports our model. They also investigated ATP levels in these cells and find that they are comparable to wild type suggesting that either mitochondria in these cells are less efficient in producing ATP although they are consuming more oxygen or that the cell consumes more ATP and thus a surplus of respiration is needed to maintain the same ATP levels. Similarly, increased oxygen consumption and even reduced ATP levels were found in fibroblasts of KRS patients carrying a mutation in ATP13A2 and also in this case an increased mitochondrial mass, as seen by increased mtDNA copy numbers, was reported (Grunewald et al., 2012). The fact that loss of function of ATP13A2 in cell culture affects mitochondrial function in a very similar way than deletion of catp- 6 in C. elegans suggests that the two proteins indeed are orthologs exhibiting similar cellular functions. 
To conclude, mitochondrial membrane potential is compromised in catp- 6 mutants indicating that mitochondrial function is impaired. Moreover, we observed changes in the composition of complex IV and it remains to be determined whether those might account for functional deficits. Intriguingly, the mutants displayed increased oxygen consumption rates possibly due to increased mitochondrial mass. Whether deletion of catp-6, a lysosomal ATPase, affects mitochondrial degradation and thereby causes accumulation dysfunctional mitochondria will be the subject of future studies.

\subsection{5. catp-6 mutants show an increased sensitivity towards oxidative stress, activation of the mitochondrial stress response and a reduced lifespan}

We have found that catp- 6 mutants exhibit an increased sensitivity towards oxidative stress induced by paraquat and also see a strong expression of the mitochondrial chaperone $h s p-6$ under basal conditions without extrinsically inducing oxidative stress indicating that the mitochondrial stress response is intrinsically activated. The mitochondrial stress response is induced by high loads of misfolded proteins in the mitochondria and leads to increased expression of the mitochondrial chaperone $h s p-6$ (C. elegans ortholog of the mitochondrial form of mammalian Hsp70) which helps to restore proper folding of the proteins (Yoneda et al., 2004). The fact that this pathway is activated in catp-6 deletion mutants indicates that enhanced misfolding of mitochondrial proteins takes place. This could be caused by increased production of reactive oxygen species (ROS) as oxidative stress causes oxidative damage to proteins which can perturb proper folding. It was shown that the majority of ROS in the cell is generated at complexes I and III of the respiratory chain (Chen et al., 2003b; St-Pierre et al., 2002) and that dysfunction or inhibition of several components of the respiratory chain leads to higher amounts of ROS being produced (Kudin et al., 2004). As we have seen changes in the composition of complex IV and a markedly reduced membrane potential in catp- 6 mutants, it is possible that mitochondrial dysfunction of the deletion mutants is accompanied with an increased production of ROS and thus more oxidative damage to mitochondrial proteins and induction of the mitochondrial unfolded protein response.

Also the increased sensitivity to oxidative stress induced by treatment with paraquat is in line with increased ROS levels in catp- 6 mutants. If the basal ROS production is increased in the mutants, the ROS generated by paraquat are adding onto these basal levels so that a critical threshold that cannot be handled anymore by the cellular antioxidant systems is reached earlier. We are currently working on determining whether the level of ROS produced by catp- 6 mutants is indeed increased when compared to wild type. This can either be done by 
the use of fluorescent probes like 2,7-dichlorodihydrofluorescein-diacetate (H2-DCF-DA) that detect ROS (Schulz et al., 2007) or by expression of mitochondrially-targeted roGFP, a GFP variant that is sensitive to oxidative stress and has a different emission spectrum in the reduced than in the oxidized state (Dooley et al., 2004; Morgan et al., 2011). It is possible that the increase in ROS levels is not very prominent under basal conditions as several antioxidant systems exist in the cell like superoxide dismutases (SODs), catalases, glutathione (GSH), GSH peroxidase and GSH reductase that help to detoxify the ROS thus preventing massive damage to cellular macromolecules. However, when these systems are already fully challenged under basal conditions, additional induction of oxidative stress by treatment with paraquat will overwhelm the system so that not all ROS cannot be cleared as seen by the increased sensitivity of catp- 6 mutants to paraquat. Therefore, besides measuring ROS levels in the mutants, it will also be interesting to investigate the activity of the antioxidant systems as an increase in their activity could hint at increased ROS production even if the basal ROS levels are not changed significantly. In this context we are currently working on generating transgenic animals expressing a sod-3::gfp reporter for mitochondrial superoxide dismutase activity in wild type worms and catp-6 mutants. This reporter will tell us whether SOD activity is intrinsically activated in the mutants due to increased ROS production and if this is not the case it will also be a valuable tool to determine whether SOD activity in the mutants can be induced upon induction of oxidative stress. This is interesting as an alternative explanation for increased ROS levels in the mutants could be failure of ROS scavenging instead of increased ROS generation.

If our hypothesis that catp- 6 mutants have a higher load of ROS is correct, this would also be an explanation for the reduction in lifespan of the mutants. Back in 1956 Harman postulated for the first time that free radicals play a major role in determining the life span of an organism (Harman, 1956). Later on, it was assumed that the respiratory rate could be negatively correlated to life span as several long lived mutants were found to exhibit a slow respiratory rate and as less ROS will be produced by decreased respiratory chain activity (Dillin et al., 2002; Van Voorhies, 2002). The identification of mutants that - although exhibiting reduced metabolic rates - show a reduction in life span (Hartman et al., 2001; Kayser et al., 2004) disproved this hypothesis. The two mutants that were exhibiting decreased respiratory activity and shortened lifespan in C. elegans are gas-1 and mev-1, components of complexes I and II, respectively (Ishii et al., 1998; Kayser et al., 1999). Remarkably, both mutants were found to exhibit higher levels of ROS when compared to wild type (Kayser et al., 2004; Senoo-Matsuda et al., 2001), with high levels of ROS having a negative impact on life span. Further support for this theory came from the observation that treatment of $C$. elegans with SOD and catalase mimetics or antioxidants such as Coenzyme Q10 or Vitamin E can confer 
a life span extension (Ishii et al., 2004; Melov et al., 2000). Nevertheless, the impact of ROS on lifespan seems to be more complicated as it was recently proposed, that low levels of ROS are important for several signaling cascades and that therefore complete abolishment of ROS could also have detrimental effects for the cell [reviewed in (Sohal and Orr, 2012)]. Interestingly, one of the above mentioned mutants, mev-1, shares several phenotypes of catp-6 mutants. Besides reduced life span and increased sensitivity to oxidative stress, mev-1 worms are also characterized by a strongly reduced brood size (Ishii et al., 1990) and a loss of mitochondrial membrane potential (Senoo-Matsuda et al., 2003). It is quite remarkable that deletion of the lysosomal P-type ATPase catp-6 leads to similar effects than loss of a component of the mitochondrial respiratory chain. It will be interesting to see whether the effects of catp-6 deletion are also acting via dysfunction of complex II of the respiratory chain. Moreover, we are planning to investigate mitochondrial morphology in mev-1 mutants to see whether also mitochondrial morphology and DRP-1 localization are similarly affected in the two mutants.

In conclusion, we find that catp- 6 mutants display increased sensitivity to oxidative stress as well as induction of the mitochondrial stress response suggesting that ROS production is increased in the mutants which might also explain the shortened life span.

\subsubsection{Increased activation of AMPK is not accompanied by increased basal autophagy}

As we had observed both morphological and functional defects in mitochondria of catp-6 mutants, we were aiming in understanding the impact of these changes on cellular function and homeostatic signaling. As mitochondrial dysfunction is usually accompanied by a decrease in cellular ATP levels, we were reasoning that this could lead to induction of AMP-activated protein kinase (AMPK), which is phosphorylated - and thereby activated - at Thr172 by LKB1 (PAR-4 in C. elegans) in response to decreased ATP levels and thus a rise in the AMP/ATP ratio (Hawley et al., 1996; Lee et al., 2008; Shaw et al., 2004). In line with our hypothesis that mitochondrial dysfunction causes a reduction in ATP production, we found higher levels of phosphorylated AMPK in catp-6 mutants. Interestingly, it was shown that AMPK can also be activated by ROS either directly or via ATM (ataxia telangiectasia mutated), a factor that normally is involved in the cellular damage response and resides in the nucleus but that is translocated to the cytoplasm during oxidative stress [reviewed in (ScherzShouval and Elazar, 2011)]. Cytoplasmic ATM then activates AMPK and thereby induces autophagy [reviewed in (Scherz-Shouval and Elazar, 2011)]. Whether increased phosphory- 
lation of AMPK in catp- 6 mutants occurs in response to ATP depletion or as a result of increased oxidative stress or a combination needs to be assessed in future studies. As we are planning to determine both ATP and ROS levels in the mutants this might help to get an idea what is causative for AMPK activation.

AMPK is an important regulator of cellular metabolism and its activation leads to inhibition of anabolic processes while catabolic pathways are stimulated [reviewed in (Hardie, 2004; Mihaylova and Shaw, 2011)]. Activated AMPK leads to induction of autophagy by inhibition of the mTOR (mammalian target of Rapamycin)/Raptor complex which itself has an inhibitory effect on autophagy [reviewed in (Jung et al., 2010)]. Besides inhibiting mTOR, phosphorylated AMPK can also stimulate autophagy via activation of ULK1 (mammalian ortholog of yeast Atg1), a serine-threonine kinase involved in initiation of autophagy (Egan et al., 2011b; Kim et al., 2011). In line with increased AMPK phosphorylation we found more autophagic foci in eggs of catp- 6 mutants. It remains to be determined whether this is indeed caused by increased induction of autophagy as a decreased clearance of autophagosomes could also explain this effect. While we could easily detect increased amounts of autophagic foci in embryos of catp- 6 mutants, the effect was not as clear in older worms. This could be due to the fact that embryos in which autophagy is strongly induced are not surviving and die at early stages. In line with this we have found morphologically abnormal eggs in catp-6 mutants that most probably die as embryos. It is thus conceivable that those worms that are surviving have developed compensatory mechanisms that prevent excessive autophagic activity that could lead to cell death. Conflicting data has been published about the effects of knockdown of ATP13A2, the human ortholog of catp-6, in cell culture. While Ramonet et al. reported increased clearance of autophagosomes but no change in induction of autophagy upon loss of ATP13A2 (Ramonet et al., 2012), another study described that KD of ATP13A2 leads to a defect in both autophagy induction and autophagic degradation so that basal levels of autophagy are not changed (Gusdon et al., 2011). The fact that both studies do not see a change of basal autophagy is in line with our observation in catp- 6 mutants at postembryonic stages and a more detailed examination of autophagic degradation and induction processes is required to fully understand the role of CATP-6 in regulation of autophagy.

Interestingly, besides its role in regulating macroautophagy, AMPK has also been linked to mitochondrial degradation. Activation of ULK1 by AMPK has been attributed an important role in the regulation of mitophagy (a specialized form of autophagy) and loss of ULK1 as well as loss of the AMPK recognition motif in ULK1 leads to impaired function and morphology of mitochondria (Egan et al., 2011a; Egan et al., 2011b). Whether increased activation of AMPK is involved in mediating mitochondrial dysfunction and disturbing mitochondrial dy- 
namics in catp- 6 mutants has to be determined. Furthermore, it would be interesting to specifically analyze mitophagy in catp-6 mutants, however, such markers are currently not available in C. elegans. Apart from inducing mitochondrial degradation, in mammals, AMPK can also stimulate mitochondrial biogenesis by up-regulating PGC-1a (peroxisome-proliferatoractivated receptor coactivator $1 \alpha$ ), a transcription coactivator involved in regulating cellular energy metabolism [reviewed in (Hardie, 2011)]. This is particularly interesting as we have hypothesized that mitochondrial mass is increased in catp- 6 mutants and increased mitochondrial biogenesis mediated by activation of AMPK could explain this effect.

In summary, we have found increased induction of autophagy in embryos lacking CATP-6 function. This up-regulation of autophagy is likely to be caused by increased AMPK activity in catp- 6 mutants. During larval development and in adult catp- 6 mutant worms, autophagy was only slightly elevated despite increased levels of active phospho-AMPK. It is therefore likely that compensatory mechanisms prevent chronic activation of autophagy as excessive autophagy leads to cell death (Kang et al., 2007). Besides its stimulatory effect on autophagy, AMPK is involved in the regulation of many other cellular processes including mitochondrial biogenesis and degradation via mitophagy. Thus, it will be interesting to determine whether activation of AMPK plays a role in mediating the effects of catp- 6 deletion on mitochondrial function and morphology.

\subsubsection{Deletion of the lysosomal P-type ATPase CATP-6 causes mitochondrial defects - novel link between lysosomes and mitochondria}

Our results have shown that deletion of the lysosomal P-type ATPase has a strong impact on both mitochondrial function and morphology. The fact that only the mitochondrial stress response was activated in the mutants but no induction of other cellular stress responses was observed, indicates that loss of function of CATP-6 rather specifically affects mitochondria. It was previously reported that lysosomal dysfunction in lysosomal storage diseases causes mitochondrial defects including fragmentation and impaired $\mathrm{Ca}^{2+}$-buffering capacity (Jennings et al., 2006). Disturbance of lysosomal function has furthermore been shown to cause accumulation of autophagosomes due to both increased autophagy activation and decreased clearance (Cao et al., 2006; Fukuda et al., 2006; Settembre et al., 2008; Vergarajauregui et al., 2008). As the clearance of damaged mitochondria relies on autophagic degradation [reviewed in (Wang and Klionsky, 2011)] and given the autophagic defects caused by lysosomal dysfunction, disturbances of mitophagy could be the cause for the mitochondrial defects seen in deletion mutants of the lysosomal P-type ATPase CATP-6. In line with this, it was 
shown that blocking autophagy in yeast causes dysfunctional mitochondria to accumulate and leads to increased ROS levels (Zhang et al., 2007). Furthermore, mitochondrial dysfunction due to defective mitophagy has been implicated in the pathogenesis of PD as mutations in the PD-related genes PINK1 and Parkin which are involved in targeting damaged mitochondria for mitophagy (Narendra et al., 2008; Narendra et al., 2010), cause mitochondrial defects (Clark et al., 2006; Greene et al., 2003; Park et al., 2006; Yang et al., 2006). Due to the lack of specific markers for mitophagy it is to date not possible to directly assess whether catp- 6 mutants display defects in mitophagy. Basal levels of autophagy though seem not to be affected in catp-6 mutant worms at postembryonic stages (preliminary results, data not shown) and were also not seen in cell culture models using siRNA against the human ortholog ATP13A2 (Gusdon et al., 2011; Ramonet et al., 2012). It is still possible that autophagic flux is changed in the mutants but Gusdon et al. also demonstrated that lysosomal stability and $\mathrm{pH}$ are not affected by KD of ATP13A2 indicating that degradation in the lysosomes is presumably working. Recent data suggests that lysosomes, besides their degradative function are also part of the cellular signaling network with the mTOR complex 1 (mTORC1) being translocated to the lysosomal membrane and activated in the presence of amino acids causing inhibition of autophagy (Zoncu et al., 2011). Interestingly, a lysosomal v-ATPase is required for amino acid induced activation of mTOR (Zoncu et al., 2011) indicating that lysosomes not only are required for autophagosomal clearance but are also involved in the regulation of autophagy. Furthermore, Korolchuk et al. have demonstrated that lysosomal positioning is also an important factor controlling autophagy. While lysosomes are located in the periphery of the cell when nutrients are available, upon starvation they are transported to the perinuclear region, where also most of the autophagosomes are accumulating. This leads to decreased mTORC1 activity and stimulates both autophagosome formation and fusion of autophagosomes with lysosomes for degradation (Korolchuk et al., 2011). As mTORC1 activity has also been implicated in the control of mitochondrial function (Cunningham et al., 2007; Koyanagi et al., 2011), mTORC1 might be the missing link between loss of function of the lysosomal protein CATP- 6 and severe mitochondrial dysfunction and needs to be subjected to future investigations. 


\section{Materials and methods}

\subsection{Molecular cloning}

Standard molecular biology techniques were used for cloning. A list of all constructs generated including cloning strategy and primers used for the PCR is provided in the appendix (Table 8.2).

\section{PCR}

PCR on the genes of interest was performed using Taq Polymerase (Invitrogen; for constructs $<1 \mathrm{~kb}$ ) or PfuUltra II Polymerase (Stratagene; for constructs $>1 \mathrm{~kb}$ ) according to the manufacturer's instructions. The primers that were used in this study are listed in Table 8.3 in the Appendix.

\section{Restriction digests}

Restriction enzymes were purchased from Fermentas. For preparative digests 1 ug DNA was either cut with $0.5 \mu \mathrm{L}$ of the respective enzymes and incubated OVN at $37^{\circ} \mathrm{C}$ (except for enzymes that have different optimal temperatures) or with 1-2 $\mu \mathrm{L}$ of the enzymes and incubated for $3 \mathrm{~h}$ at the same temperature. Digestion buffers were used according to the manufacturer's instructions. Depending on the amount of enzyme used, digests were performed in 20-50 $\mu \mathrm{L}$ total volume (enzyme should not account for more than $10 \%$ of the total volume). Digests were then run on a 1\% agarose gel in 1x TBE and gel extraction (kit from Fermentas) was performed for the bands of interest. Alternatively, if the digest was performed on a PCR product or if it resulted in just one cut in a plasmid resulting in linearization, the digest was purified using a PCR purification kit (Fermentas).

\section{Ligation}

For Ligations, usually a 3:1 ratio of insert to vector was used. For 3-fragment ligations, either a 3:3:1 ratio (insert 1 :insert 2:vector) was used or ratios were adjusted if there was a discrepancy of the size of the two inserts. Ligation reactions were performed in 10-20 $\mu \mathrm{L}$ final volume with 1-2 $\mu \mathrm{L}$ 10x T4 Ligase buffer (Fermentas) and $1 \mu \mathrm{L}$ T4 DNA Ligase (Fermentas). Ligations were incubated at RT for $1-3 \mathrm{~h}$ or at $16{ }^{\circ} \mathrm{C}$ OVN. For pGEM-T cloning of PCR products, ligations were performed using a kit purchased from Promega.

\section{Transformation}

Transformations were done using chemocompetent E. coli DH5a (Invitrogen). Competent cells were stored at $-80^{\circ} \mathrm{C}$ until use and thawed on ice for $10-15$ min before adding $100 \mu \mathrm{L}$ of competent cells to the ligation sample or to $1 \mu \mathrm{L}$ of the plasmid to be retransformed. After 
20-30 min of incubation of cells with the DNA on ice, cells were heat-shocked at $42{ }^{\circ} \mathrm{C}$ for 2 min followed by 5 min incubation on ice. Subsequently, $400 \mathrm{~mL}$ LB medium were added and cells were incubated for $30-60 \mathrm{~min}$ in a $37^{\circ} \mathrm{C}$ shaker. For retransformations, $80-100 \mu \mathrm{L}$ of the cell suspension were plated on LB plates containing the selection antibiotics. For transformation of ligations, cells were centrifuged at $4000 \mathrm{rpm}$ for $5 \mathrm{~min}$ and the supernatant was taken away leaving the cells in a volume of approximately $150 \mu \mathrm{L}$. Cells were resuspended by pipetting up and down and then plated on LB plates containing the appropriate selection antibiotic. For transformation of pGEM-T cloned PCR products, JM109 (Promega) competent cells were used and transformation plates (containing Amp) were treated with $10 \mu \mathrm{L} 1 \mathrm{M} \mathrm{IPTG}$ and $20 \mu \mathrm{L} \mathrm{X-Gal} \mathrm{(50} \mathrm{mg/ml)} \mathrm{before} \mathrm{plating} \mathrm{the} \mathrm{transformed} \mathrm{bacteria.}$

\section{Miniprep}

OVN cultures were prepared by inoculating $5 \mathrm{~mL}$ LB containing Ampicillin $(100 \mu \mathrm{g} / \mathrm{ml})$ with one colony each from a transformation plate and incubation OVN at $37^{\circ} \mathrm{C}$. Bacteria were centrifuged at $2500 \mathrm{rpm}$ for $10 \mathrm{~min}$. Miniprep was done using a kit from Invitrogen according to the manufacturer's instructions. In the end, DNA was eluted with $75 \mu \mathrm{L} d d \mathrm{H}_{2} \mathrm{O}$. Correct clones were identified by test digest and subsequent sequencing.

\subsection{C. elegans maintenance and genetics}

\section{C. elegans strains}

C. elegans strains were cultured on NGM (Nematode Growth Medium) plates seeded with E. coli OP50 as described previously (Brenner, 1974). Strains were kept at $20^{\circ} \mathrm{C}$ if not otherwise mentioned. Strains used in this study are listed in Table 8.5 in the appendix. Bristol N2 was used as wild type strain. Strains were ordered from the C. elegans Gene Knockout Consortium (CGC; Vancouver, Canada and Minnesota/Oklahoma, USA) and the National Biosource Project for the Nematode C. elegans (Tokyo, Japan) if not stated differently.

\section{Generation of transgenic worms by microinjection}

Microinjection of plasmid mixes into wild type worms was used to generate transgenic lines carrying extrachromosomal arrays as described by Mello \& Fire (Mello and Fire, 1995). The injection mixes were containing the plasmids of interest along with coinjection markers which allow screening for progeny of the injected worms for positives carrying an extrachromosomal array. The total DNA concentration of injection mixes was adjusted to $100 \mathrm{ng} / \mu \mathrm{L}$ by addition of pBluescript II SK(+). For injection, L4 or young adult worms were fixed in a drop of injection oil (Sigma H8898) on a dried $2 \%$ agarose pad. Injection into the distal gonads was 
performed using a micromanipulator and a pump as described earlier (Mello and Fire, 1995). After injection, worms were rescued by addition of M9 Buffer onto the pad and put onto fresh NGM plates seeded with E. coli OP50. The F1 progeny is screened for worms carrying the extrachromosomal array as seen by expression of the coinjection marker and positive progeny is separated on individual plates. Each of these positive F1 animals that produces positive F2 is considered as an independent transgenic line. A list of the injection mixes used in this study is provided in the appendix (Table 8.4) and the transgenic strains used in this study are listed in Table 8.5.

\section{Crosses}

For crossings, males of a him-8; ccls4251 strain (which carries a GFP-marker for muscle cells in a him-8 (high incidence of males 8 ) background which leads to increased occurrence of male progeny). The GFP-marker allows for the distinction of cross-progeny from selfprogeny. Alternatively, males of the marker strain to be crossed into the mutants were generated by subjecting $L 4$ worms of the marker strain to a heat shock at $30^{\circ} \mathrm{C}$ for $6 \mathrm{~h}$ which leads to occurrence of male progeny in the F1 that can subsequently be used for the crosses.

\section{Worm PCR}

Worm PCR was performed for genotyping the progeny of crosses. Primers used for worm PCRs are listed in Table 8.3. First, worm lysates were generated by picking either one worm in $10 \mu \mathrm{L}$ (for single worm PCR) or 10-20 worms into 10-20 $\mu \mathrm{L}$ lysis buffer (1x PCR buffer containing $1 \mu \mathrm{g} / \mu \mathrm{L}$ Proteinase $\mathrm{K}$ (Roth) freshly prepared from 10x Worm PCR buffer and a Proteinase $\mathrm{K}$ stock $(20 \mathrm{mg} / \mathrm{ml}))$. Worms in lysis buffer were then frozen at $-80^{\circ} \mathrm{C}$ for at least 10 min before performing worm lysis in a PCR machine. The protocol was as follows: $65^{\circ} \mathrm{C}$ for $1 \mathrm{~h}$ followed by $15 \mathrm{~min}$ at $95^{\circ} \mathrm{C}$ to inactivate proteinase $\mathrm{K} .2 \mu \mathrm{L}$ of the worm lysate where then used as template for the worm PCR. Reaction mix for worm PCR per reaction (total volume: $25 \mu \mathrm{L})$ :
Worm lysate
$2.0 \mu \mathrm{L}$
10x Worm PCR buffer
$2.5 \mu \mathrm{L}$
Forward Primer
$0.25 \mu \mathrm{L}$
Reverse Primer
$0.25 \mu \mathrm{L}$
dNTPs (Fermentas)
$0.5 \mu \mathrm{L}$
Taq A or pTC-Taq (homemade) $\quad 0.25 \mu \mathrm{L}$
$\mathrm{dH}_{2} \mathrm{O}$
$19.25 \mu \mathrm{L}$ 


\subsection{Imaging}

\section{High Pressure Freeze Electron Microscopy (HPF-EM)}

HPF-EM was done by Dr. Jan Hegerman as described previously (Sumakovic et al., 2009). Worms were subjected to HPF as young adults. For analysis of solutions containing aS, nitrocellulose capillary tubes were used. The protocol for generating tomograms of mitochondria for catp-6 worms and wild type control was described in (Witte et al., 2011). The tomograms were generated on longitudinal sections through BWMs of the worms. Analysis of cross-section areas of mitochondria for catp- 6 mutants and wild type control was performed in cross-sections through BWM cells.

\section{Preparation of worms for light microscopy}

Before imaging, worms were anaesthetized in a drop of $50 \mathrm{mM} \mathrm{NaN}_{3}$ (sodium azide) in M9 buffer on a fresh $2 \%$ agarose pad and incubated several minutes until worms were immobilized before covering with a glass coverslip (Bargmann and Avery, 1995). If not otherwise stated, young adult worms ( 3 days after the eggs have been laid) were imaged.

\section{DIC microscopy}

DIC (Differential interference contrast) images were taken at a Zeiss Axiovert 200 Microscope. DIC images of whole worms were generated by taking a series of images of different segments of the worms with a 20x objective and then merging them in Photoshop to get a picture of the full-length worm. For these images, adult worms $24 \mathrm{~h}$ post-L4 stage were used. Images of the uterus were taken form adults $48 \mathrm{~h}$ post-L4 with a $20 \mathrm{x}$ microscope and the vulva was imaged in young adult worms using a $63 x$ objective.

\section{Imaging of DA neurons}

Imaging of $C$. elegans DA neurons expressing mRFP from a dat-1 promoter was performed by Dr. Stefan Eimer using a Leica Confocal Laser Scanning Microscope SP2 and the images were scored for defects in neurite morphology.

\section{Imaging aS-mYFP aggregation in vulva muscles}

aS-mYFP aggregation was assessed by imaging the vulva musculature of 10-day-old adults on a UltraviewVOX spinning disk microscope (Perkin Elmer) with a 60x/1.35 objective. Analysis was performed on z-stacks (with a z-spacing of $2 \mu \mathrm{m}$ ) which were projected into a single plane using the extended focus tool of the Volocity software (Perkin Elmer). At least two independent transgenic lines were analyzed for each aS variant. Vulva muscles were scored positive if, at least, one fibrillar aggregate was visible. 


\section{Imaging mitochondria and DRP-1 in muscle cells and neurons}

Mitochondria in BWM cells were visualized by using the OMM marker pmyo-3::tom70-cfp (kindly provided by A. van der Bliek, UCLA; (Labrousse et al., 1999)). For analysis of DRP-1 localization, a TagRFP-DRP-1 fusion protein was expressed along with the mitochondrial marker in the muscle cells. Imaging was done on a UltraviewVOX spinning disk microscope (Perkin Elmer) with a 100x/1.40 oil immersion objective. Muscle cells were classified according to the appearance of wild type-like, thin or fragmented mitochondria. The classification was done on z-stacks (with a z-spacing of $1 \mu \mathrm{m}$ ) which were projected into a single plane using the extended focus tool of the Volocity software (Perkin Elmer). DRP-1 localization was scored as normal, if distinct spots were equally distributed throughout the cell and as clustered if larger aggregated of DRP-1 were present in proximity to the nucleus.

In order to analyze changes in mitochondrial morphology occurring during aging, we compared the mitochondrial morphology in muscle cells of young adult worms ( 3 days after hatching) with that of worms in the post-reproductive stage (7 days after hatching). This experiment was performed with two of the transgenic lines described above - one with and one without ectopic expression of $\alpha \mathrm{S}$.

For the starvation experiment, worms were transferred to special NGM plates that lack peptone and contain $100 \mu \mathrm{g} / \mathrm{ml} \mathrm{Amp} \mathrm{and} \mathrm{that} \mathrm{were} \mathrm{not} \mathrm{seeded} \mathrm{with} \mathrm{bacteria.} \mathrm{Transfer} \mathrm{of} \mathrm{the}$ worms from normal NGM plates with bacteria to the starvation plates was done when they had reached L4 or L3 stage and worms were grown on these plates for 24 and $52 \mathrm{~h}$, respectively, before imaging mitochondria in BWMs.

For visualization of mitochondria and DRP-1 in neurons, the TagRFP-DRP-1 and TOM70-CFP markers were expressed under the control of a pan-neuronal rab-3 promoter. Imaging of ventral nerve cord neurons was performed in the same way as described for the muscle cells but z-spacing in the case of neurons was set to $0.3 \mu \mathrm{m}$. Mitochondrial appearance in the neuronal cell bodies was classified into three categories: ring like, tubular or highly fragmented structures. In the case of aS-expressing worms, the aS-mYFP signal was also imaged to assess aggregation in neurons.

\section{Expression pattern analysis}

For the expression pattern analysis, GFP was expressed from the catp-6 promoter. For cloning of the promoter, the sequence spanning $4 \mathrm{~kb}$ upstream of the catp- 6 start ATG was amplified and the pcatp-6::gfp construct was generated by Katrin Schwarze, a former technician in the lab. Worms carrying an extrachromosomal array expressing this construct were generated and imaged with an UltraviewVOX spinning Disk microscope (Perkin Elmer) with a $60 x / 1.35$ oil immersion objective was used. Images were taken as z-stacks (with a z-spacing 
of $2 \mu \mathrm{m})$ and were subsequently projected into a single plane using the extended focus tool of the Volocity software (Perkin Elmer).

\section{Imaging of vulva muscles}

For imaging of the vulva muscle morphology and arrangement, a 40x/0.75 objective at the UltraviewVOX spinning disk microscope (Perkin Elmer) was used. Vulva muscles were visualized by using the strain PD4595 which carries an integrated array expressing ceh-24::gfp, a marker that has been described to label all eight vulva muscles (Harfe et al., 1998). The vulva was imaged from a lateral view. Analysis was performed on z-stacks (with a z-spacing of $2 \mu \mathrm{m}$ ) which were projected into a single plane using the extended focus tool of the Volocity software (Perkin Elmer). The vulva musculature was scored for three defects: muscles that are not properly attached to the vulva, muscle with irregular arrangement and lack of at least one muscle.

\section{Imaging of myo-filament arrangement in BWM cells}

Analysis of the myo-filament arrangement in BWM cells of catp- 6 worms and wild type controls was performed by Dr. Sabine König using an UltraviewVOX spinning Disk microscope (Perkin Elmer) with a 60x/1.35 oil immersion objective. For visualization of the myo-filaments, a translational fusion of GFP with MYO-3, a myosin heavy chain protein, was used (Meissner et al., 2009).

\section{Imaging germline apoptosis}

Apoptotic corpses in the $C$. elegans germline were visualized using the previously described reporter construct plim-7::ced-1-gfp (Schumacher et al., 2005). The gonads of young adult worms were imaged on an UltraviewVOX spinning Disk microscope (Perkin Elmer) with a 40x/0.75 oil immersion objective. Apoptotic corpses per gonad arm were counted in z-stacks with z-spacing of $2 \mu \mathrm{m}$.

\section{Imaging autophagic foci in C. elegans embryos}

Autophagic foci were visualized by using a previously described GFP-LGG-1 reporter construct driven by the lgg-1 promoter (Kang et al., 2007; Melendez et al., 2008). Eggs of the 150-300 cell stage were imaged in M9 buffer on a fresh $2 \%$ agarose pad on an UltraviewVOX spinning Disk microscope (Perkin Elmer) with a 100x/1.40 oil immersion objective. Analysis of the autophagic foci was done on z-stacks (with a z-spacing of $1 \mu \mathrm{m}$ ) which were projected into a single plane using the extended focus tool of the Volocity software (Perkin Elmer). Analysis of number and size of the autophagic foci was done in ImageJ. For this purpose, the image was converted to 8 bit and a threshold was set to create a binary image. 
Then, a built-in multiple particle analyzer was used with the following settings: size 50-100 pixels and circularity 0.3-1.0.

\subsection{RNAi by feeding}

The mitochondrial dynamics genes $d r p-1$, fis-1, fzo-1 and eat-3 were knocked down by feeding RNAi as described previously (Kamath and Ahringer, 2003). For this purpose, the genes were cloned into the RNAi vector L4440 and transformed in HT115 cells which were then seeded onto RNAi plates (NGM plates containing $100 \mu \mathrm{g} / \mathrm{ml}$ Amp and $1 \mathrm{mM}$ IPTG). The empty L4440 vector was used as negative control and RNAi against pos-1 was used as positive control as it had previously been shown that RNAi against this gene results in embryonic lethality (Kamath and Ahringer, 2003). The RNAi experiments were done in an RNAihypersensitive eri-1(mg366) background. For the RNAi experiments, the F0 generation was transferred to RNAi plates at the L3 larval stage. The mothers were subsequently transferred to new RNAi plates every $12 \mathrm{~h}$. As judged by lethality of pos-1 RNAi, RNAi was working efficiently for eggs of mothers that had been on the RNAi plates for $24 \mathrm{~h}$. Therefore, F1 progeny that had been laid in a time window between 24 and $36 \mathrm{~h}$ after transfer of the mothers to RNAi plates were analyzed. Mitochondrial morphology and DRP-1 localization in these worms was analyzed as described above.

\subsection{Phenotypic analysis/Behavioural Assays}

\section{Food slowing response}

The food slowing response of worms expressing different $\alpha S$ constructs under the control of a dat-1 promoter was analyzed by Dr. Stefan Eimer. The slowing rate was determined as the average decrease in movement (body bends/min) for animals placed on food as compared to animals without food.

\section{Brood size}

Brood size analysis of catp- 6 deletion mutants and wild type controls was performed by Dr. Sabine König. L4 worms were separated onto individual plates and each worm was transferred to a new plate every 12-24 $\mathrm{h}$ and the progeny of the previous plate was counted. Besides determining the brood size, worms were scored for Egl and pVul phenotypes.

\section{PED}

To determine the duration of postembryonic development (PED) of catp-6 mutants and wild type controls, young adult worms were allowed to lay eggs for $4 \mathrm{~h}$. After $12 \mathrm{~h}$, hatched L1 larvae were separated onto individual NGM plates and it was determined how long it takes 
until they reach adulthood and are producing eggs themselves. For this purpose, the worms were analyzed every six hours.

\section{Movement}

Movement of catp- 6 mutants and controls was analyzed at the L3 larval stage and in young adult worms. For this analysis, worms were transferred on NGM plates without food. After an adaptation period of $30 \mathrm{~min}$, movement was analyzed by counting the number of body bends (complete sinusoidal wave) per minute. This was done by visual inspection under a dissection microscope. For each animal, the body bends were counted for a 1 min interval in three consecutive trials.

\section{Paraquat}

The sensitivity to oxidative stress was assessed by treatment with paraquat, which causes massive generation of superoxide (Bus and Gibson, 1984), as described previously (Samann et al., 2009). Paraquat plates were produced by adding $200 \mu \mathrm{L}$ of a $150 \mathrm{mM}$ paraquat solution in M9 buffer onto small $(3.5 \mathrm{~cm}$ in diameter) NGM plates seeded with E. coli OP50. Worms were transferred to paraquat plates at the L4 larval stage and survival of the worms was assessed after 2 days.

\section{Lifespan}

As catp- 6 worms have a strong Egl phenotype, they often die because their progeny is hatching inside the mother, eventually killing it. In order to assess lifespan independent of this, we were performing lifespan analyses in a glp-4(bn2) background, a temperaturesensitive allele in which germline proliferation is inhibited at the non-permissive temperature of $25^{\circ} \mathrm{C}$ (Beanan and Strome, 1992). For the lifespan assay, 3 plates of $15 \mathrm{~L} 2$ worms were prepared per strain and the plates were kept at $25^{\circ} \mathrm{C}$ until the worms reached adulthood so that no gonads were formed. Then, the worms were grown at $20^{\circ} \mathrm{C}$ and survival was assessed every day.

\subsection{Analysis of mitochondrial function}

\section{Isolation of $C$. elegans mitochondria}

For mitochondrial isolation, worms were grown on big $(12 \times 12 \mathrm{~cm})$ double peptone NGM plates (peptone concentration doubled when compared to normal NGM plates) which were seeded with a fast-growing E. coli OP50 strain generating a thick bacterial lawn. For each strain, 60 plates were prepared and worms were washed with M9 buffer when the plate was full of worms, shortly before starvation. Worms were collected in $15 \mathrm{~mL}$ Falcon tubes and centrifuged for $5 \mathrm{~min}$ at $800 \mathrm{rpm}$ before taking off the supernatant. Subsequently, worms 
were washed twice in M9 buffer and the pellets were brought to the lab of Prof. Peter Rehling where Dr. Jan Dudek was performing the isolation of the mitochondria.

\section{Membrane potential}

The membrane potential of isolated mitochondria was measured by Dr. Jan Dudek. Mitochondria were incubated in isoosmotic potassium buffer in the presence of $1 \mu \mathrm{M} \mathrm{DiSC} 3(5)$. Fluorescence of this dye is quenched by the mitochondrial membrane potential. The specificity of the measurement was assessed by $\mathrm{KCN}$ (potassium cyanide; $1 \mathrm{M}$ in $\mathrm{H}_{2} \mathrm{O}$ ) inhibition of complex IV of the respiratory chain. The membrane potential was calculated as the difference between mitochondrial quenching of active and KCN-intoxicated mitochondria.

\section{Analysis of respiratory chain complex IV activity}

Analysis of the activity and composition of complex IV of the respiratory chain was done by Dr. Jan Dudek. Shortly, mitochondrial respiratory chain complexes were solubilized by Blue Native polyacrylamide gel electrophoresis (BN-PAGE). For staining of complex IV, reduced cytochrome c (cyt c) was added in the gel. Oxidation of cyt $\mathrm{c}$ by complex IV is supported by the diaminobenzidine specifically reducing cytochrome c. Thereby generated oxidized diaminobenzidine falls out as a brown precipitate, thus staining for complex IV activity.

\section{Oxygen consumption}

Oxygen consumption was measured in whole worms using a Seahorse Flux Analyzer XF96. The experiment was performed in 96-well plates and for each well, 20 young adult worms of the respective strains were picked into $180 \mu \mathrm{L}$ M9 buffer. Measurements were done at 20-25 ${ }^{\circ} \mathrm{C}$. First, the basal oxygen consumption rate (OCR) was determined. Then, the mitochondrial uncoupler FCCP was added to a final concentration of $10 \mu \mathrm{M}$ followed by another measurement of the OCR and finally, the OCR after inhibition of complex IV with sodium azide $\left(\mathrm{NaN}_{3}\right)$ was assessed.

\subsection{Assessing induction of the cellular stress responses}

Induction of the cellular stress responses was assessed using GFP-reporter constructs and determining GFP expression by fluorescence microscopy using a Keyence BZ9000 microscope and by Western Blot using a monoclonal anti-GFP antibody (Roche).

\section{Mitochondrial stress response}

For the mitochondrial stress response, a hsp-6::gfp reporter strain (SJ4100) was used (Yoneda et al., 2004) and for induction of the mitochondrial stress response, L3 worms were 
incubated for $24 \mathrm{~h}$ on NGM plates containing paraquat. Paraquat plates were produced by adding $200 \mu \mathrm{L}$ of a $150 \mathrm{mM}$ paraquat solution in $\mathrm{M} 9$ buffer onto small $(3.5 \mathrm{~cm}$ in diameter) NGM plates seeded with E. coli OP50.

\section{ER unfolded protein response (UPR ${ }^{\mathrm{ER}}$ )}

For the ER stress response, the strain SJ4005 expressing a hsp-4::gfp reporter construct was used (Calfon et al., 2002) and for induction of the UPR ${ }^{\mathrm{ER}}$, worms were grown on plates containing $2 \mu \mathrm{g} / \mathrm{ml}$ Tunicamycin as described previously (Durieux et al., 2011; Yoneda et al., 2004).

\section{Cytosolic unfolded protein response (UPR ${ }^{\text {cyt}}$ )}

For the cytosolic stress response, a hsp-16.2::gfp reporter strain (CL2070) was used (Link et al., 1999) and for induction of the UPR ${ }^{\text {cyt }}$, worms were subjected to a heat-shock for $6 \mathrm{~h}$ at $30{ }^{\circ} \mathrm{C}$ as described previously (Durieux et al., 2011).

\subsection{Western blotting}

\section{a-synuclein}

aS expression levels were determined by Western Blot. Worm lysates were prepared by lysis of 30 young adult worms in PAGE buffer containing $\beta$-Mercaptoethanol. $15 \mu \mathrm{L}$ of these lysates were cooked at $95^{\circ} \mathrm{C}$ for 10 minutes followed by vortexing and centrifugation to remove debris. Samples were separated on a 12\% SDS-PAGE gel and transferred to a nitrocellulose membrane and probed against $\alpha S$ using either a 1:750 dilution of a polyclonal rabbit anti-aS antibody (Anaspec) or a 1:50 dilution of a monoclonal rat 15G7 anti-aS antibody ((Kahle et al., 2000); kindly provided by Prof. Christian Haass). A horse radish peroxidasecoupled secondary anti-rat antibody (Jackson) was used in 1:10000 dilution. Images were quantified using ImageJ and normalized to the tubulin loading control which was probed with the monoclonal mouse anti-tubulin antibody $12 \mathrm{G} 10$ (DSHB) in a 1:1000 dilution and a horse radish peroxidase-coupled secondary anti-mouse antibody (Jackson) in 1:10000 dilution.

\section{GFP}

Western Blot to determine the expression levels of the cellular stress response reporters (as described above) was done as described above for $\alpha S$ but in this case a mouse monoclonal anti-GFP antibody (Roche) was used in a 1:1000 dilution as primary antibody, again, tubulin was used as loading control. 


\section{Phosho-AMPK}

For determination of the levels of phosphorylated AMPK, worms were grown on three big NGM plates and harvested by washing off with M9 buffer shortly before starvation. The worm pellet was washed twice with M9 buffer to eliminate bacteria from the solution and was then resuspended in 1x PAGE buffer with $\beta$-Mercaptoethanol. The Western Blot was described as described above for $\alpha S$ but a 1:500 dilution of a phosphorylation specific antibody (Thr172) for $A M P K$ was used as primary $A B$ in this case $(40 \mathrm{H} 9$, monoclonal rabbit anti $p-A M P K$; Cell Signaling). Again, tubulin served as loading control. 


\section{References}

Abou-Sleiman, P.M., Muqit, M.M., McDonald, N.Q., Yang, Y.X., Gandhi, S., Healy, D.G., Harvey, K., Harvey, R.J., Deas, E., Bhatia, K., et al. (2006). A heterozygous effect for PINK1 mutations in Parkinson's disease? Ann Neurol 60, 414-419.

Alexander, C., Votruba, M., Pesch, U.E., Thiselton, D.L., Mayer, S., Moore, A., Rodriguez, M., Kellner, U., Leo-Kottler, B., Auburger, G., et al. (2000). OPA1, encoding a dynamin-related GTPase, is mutated in autosomal dominant optic atrophy linked to chromosome 3q28. Nat Genet 26, 211-215.

Andres-Mateos, E., Perier, C., Zhang, L., Blanchard-Fillion, B., Greco, T.M., Thomas, B., Ko, H.S., Sasaki, M., Ischiropoulos, H., Przedborski, S., et al. (2007). DJ-1 gene deletion reveals that DJ-1 is an atypical peroxiredoxin-like peroxidase. Proc Natl Acad Sci U S A 104, 14807-14812.

Apostolova, N., Blas-Garcia, A., and Esplugues, J.V. (2011). Mitochondria sentencing about cellular life and death: a matter of oxidative stress. Curr Pharm Des 17, 4047-4060.

Aroian, R.V., Koga, M., Mendel, J.E., Ohshima, Y., and Sternberg, P.W. (1990). The let-23 gene necessary for Caenorhabditis elegans vulval induction encodes a tyrosine kinase of the EGF receptor subfamily. Nature 348, 693-699.

Auluck, P.K., Chan, H.Y., Trojanowski, J.Q., Lee, V.M., and Bonini, N.M. (2002). Chaperone suppression of alpha-synuclein toxicity in a Drosophila model for Parkinson's disease. Science 295, 865-868.

Axelsen, K.B., and Palmgren, M.G. (1998). Evolution of substrate specificities in the P-type ATPase superfamily. J Mol Evol 46, 84-101.

Baba, M., Nakajo, S., Tu, P.H., Tomita, T., Nakaya, K., Lee, V.M., Trojanowski, J.Q., and Iwatsubo, T. (1998). Aggregation of alpha-synuclein in Lewy bodies of sporadic Parkinson's disease and dementia with Lewy bodies. Am J Pathol 152, 879-884.

Bargmann, C.I., and Avery, L. (1995). Laser killing of cells in Caenorhabditis elegans. Methods Cell Biol 48, 225-250.

Beanan, M.J., and Strome, S. (1992). Characterization of a germ-line proliferation mutation in C. elegans. Development 116, 755-766.

Beckman, K.B., and Ames, B.N. (1998). The free radical theory of aging matures. Physiol Rev 78, 547-581.

Behrens, M.I., Bruggemann, N., Chana, P., Venegas, P., Kagi, M., Parrao, T., Orellana, P., Garrido, C., Rojas, C.V., Hauke, J., et al. (2010). Clinical spectrum of Kufor-Rakeb syndrome in the Chilean kindred with ATP13A2 mutations. Mov Disord 25, 1929-1937.

Beilina, A., Van Der Brug, M., Ahmad, R., Kesavapany, S., Miller, D.W., Petsko, G.A., and Cookson, M.R. (2005). Mutations in PTEN-induced putative kinase 1 associated with recessive parkinsonism have differential effects on protein stability. Proc Natl Acad Sci U S A 102, 5703-5708.

Benedetti, C., Haynes, C.M., Yang, Y., Harding, H.P., and Ron, D. (2006). Ubiquitin-like protein 5 positively regulates chaperone gene expression in the mitochondrial unfolded protein response. Genetics 174, 229-239.

Benedetto, A., Au, C., Avila, D.S., Milatovic, D., and Aschner, M. (2010). Extracellular dopamine potentiates $\mathrm{mn}$-induced oxidative stress, lifespan reduction, and dopaminergic neurodegeneration in a BLI-3-dependent manner in Caenorhabditis elegans. PLoS Genet 6. 
Bereiter-Hahn, J., and Voth, M. (1994). Dynamics of mitochondria in living cells: shape changes, dislocations, fusion, and fission of mitochondria. Microsc Res Tech 27, 198-219.

Biere, A.L., Wood, S.J., Wypych, J., Steavenson, S., Jiang, Y., Anafi, D., Jacobsen, F.W., Jarosinski, M.A., Wu, G.M., Louis, J.C., et al. (2000). Parkinson's disease-associated alpha-synuclein is more fibrillogenic than beta- and gamma-synuclein and cannot cross-seed its homologs. J Biol Chem 275, 34574-34579.

Biskup, S., Moore, D.J., Celsi, F., Higashi, S., West, A.B., Andrabi, S.A., Kurkinen, K., Yu, S.W., Savitt, J.M., Waldvogel, H.J., et al. (2006). Localization of LRRK2 to membranous and vesicular structures in mammalian brain. Ann Neurol 60, 557-569.

Bonifati, V., Rizzu, P., Squitieri, F., Krieger, E., Vanacore, N., van Swieten, J.C., Brice, A., van Duijn, C.M., Oostra, B., Meco, G., et al. (2003a). DJ-1( PARK7), a novel gene for autosomal recessive, early onset parkinsonism. Neurol Sci 24, 159-160.

Bonifati, V., Rizzu, P., van Baren, M.J., Schaap, O., Breedveld, G.J., Krieger, E., Dekker, M.C., Squitieri, F., Ibanez, P., Joosse, M., et al. (2003b). Mutations in the DJ-1 gene associated with autosomal recessive early-onset parkinsonism. Science 299, 256-259.

Braak, H., Ghebremedhin, E., Rub, U., Bratzke, H., and Del Tredici, K. (2004). Stages in the development of Parkinson's disease-related pathology. Cell Tissue Res 318, 121-134.

Braungart, E., Gerlach, M., Riederer, P., Baumeister, R., and Hoener, M.C. (2004). Caenorhabditis elegans MPP+ model of Parkinson's disease for high-throughput drug screenings. Neurodegener Dis $1,175-183$

Brenner, S. (1974). The genetics of Caenorhabditis elegans. Genetics 77, 71-94.

Broadley, S.A., and Hartl, F.U. (2008). Mitochondrial stress signaling: a pathway unfolds. Trends Cell Biol 18, 1-4.

Brodal, P. (2004). The Central Nervous System - Structure and Function, 3rd edition edn (New York, Oxford University Press).

Brown, P. (2006). Bad oscillations in Parkinson's disease. J Neural Transm Suppl, 27-30.

Bueler, H. (2009). Impaired mitochondrial dynamics and function in the pathogenesis of Parkinson's disease. Exp Neurol 218, 235-246.

Burbulla, L.F., Krebiehl, G., and Kruger, R. (2010). Balance is the challenge--the impact of mitochondrial dynamics in Parkinson's disease. Eur J Clin Invest 40, 1048-1060.

Bus, J.S., and Gibson, J.E. (1984). Paraquat: model for oxidant-initiated toxicity. Environ Health Perspect 55, 37-46.

Calfon, M., Zeng, H., Urano, F., Till, J.H., Hubbard, S.R., Harding, H.P., Clark, S.G., and Ron, D. (2002). IRE1 couples endoplasmic reticulum load to secretory capacity by processing the XBP-1 mRNA. Nature 415, 92-96.

Canet-Aviles, R.M., Wilson, M.A., Miller, D.W., Ahmad, R., McLendon, C., Bandyopadhyay, S., Baptista, M.J., Ringe, D., Petsko, G.A., and Cookson, M.R. (2004). The Parkinson's disease protein DJ-1 is neuroprotective due to cysteine-sulfinic acid-driven mitochondrial localization. Proc Natl Acad Sci U S A 101, 9103-9108.

Cao, S., Gelwix, C.C., Caldwell, K.A., and Caldwell, G.A. (2005). Torsin-mediated protection from cellular stress in the dopaminergic neurons of Caenorhabditis elegans. J Neurosci 25, 3801-3812.

Cao, Y., Espinola, J.A., Fossale, E., Massey, A.C., Cuervo, A.M., MacDonald, M.E., and Cotman, S.L. (2006). Autophagy is disrupted in a knock-in mouse model of juvenile neuronal ceroid lipofuscinosis. $J$ Biol Chem 281, 20483-20493. 
Carmine Belin, A., Westerlund, M., Bergman, O., Nissbrandt, H., Lind, C., Sydow, O., and Galter, D. (2007). S18Y in ubiquitin carboxy-terminal hydrolase L1 (UCH-L1) associated with decreased risk of Parkinson's disease in Sweden. Parkinsonism Relat Disord 13, 295-298.

Cereghetti, G.M., Stangherlin, A., Martins de Brito, O., Chang, C.R., Blackstone, C., Bernardi, P., and Scorrano, L. (2008). Dephosphorylation by calcineurin regulates translocation of Drp1 to mitochondria. Proc Natl Acad Sci U S A 105, 15803-15808.

Cerveny, K.L., Tamura, Y., Zhang, Z., Jensen, R.E., and Sesaki, H. (2007). Regulation of mitochondrial fusion and division. Trends Cell Biol 17, 563-569.

Chan, C.S., Guzman, J.N., Ilijic, E., Mercer, J.N., Rick, C., Tkatch, T., Meredith, G.E., and Surmeier, D.J. (2007). 'Rejuvenation' protects neurons in mouse models of Parkinson's disease. Nature 447, $1081-1086$.

Chan, D.C. (2006). Mitochondrial fusion and fission in mammals. Annu Rev Cell Dev Biol 22, 79-99.

Chandra, S., and Südhof, T. (2008). alpha-synuclein, CSPalpha, SNARES and neuroprotection in vivo. In Parkinson's disease: molecular and therapeutic insights from model systems, R. Nass, and S. Przedborski, eds. (Elsevier Inc.).

Chang, C.R., and Blackstone, C. (2007). Cyclic AMP-dependent protein kinase phosphorylation of Drp1 regulates its GTPase activity and mitochondrial morphology. J Biol Chem 282, 21583-21587.

Chartier-Harlin, M.C., Kachergus, J., Roumier, C., Mouroux, V., Douay, X., Lincoln, S., Levecque, C., Larvor, L., Andrieux, J., Hulihan, M., et al. (2004). Alpha-synuclein locus duplication as a cause of familial Parkinson's disease. Lancet 364, 1167-1169.

Chen, C.M., Lin, C.H., Juan, H.F., Hu, F.J., Hsiao, Y.C., Chang, H.Y., Chao, C.Y., Chen, I.C., Lee, L.C., Wang, T.W., et al. (2011). ATP13A2 variability in Taiwanese Parkinson's disease. Am J Med Genet B Neuropsychiatr Genet 156B, 720-729.

Chen, H., and Chan, D.C. (2004). Mitochondrial dynamics in mammals. Curr Top Dev Biol 59, 119144.

Chen, H., and Chan, D.C. (2006). Critical dependence of neurons on mitochondrial dynamics. Curr Opin Cell Biol 18, 453-459.

Chen, H., and Chan, D.C. (2009). Mitochondrial dynamics--fusion, fission, movement, and mitophagy-in neurodegenerative diseases. Hum Mol Genet 18, R169-176.

Chen, H., Detmer, S.A., Ewald, A.J., Griffin, E.E., Fraser, S.E., and Chan, D.C. (2003a). Mitofusins Mfn1 and Mfn2 coordinately regulate mitochondrial fusion and are essential for embryonic development. J Cell Biol 160, 189-200.

Chen, L., and Feany, M.B. (2005). Alpha-synuclein phosphorylation controls neurotoxicity and inclusion formation in a Drosophila model of Parkinson disease. Nat Neurosci 8, 657-663.

Chen, Q., Vazquez, E.J., Moghaddas, S., Hoppel, C.L., and Lesnefsky, E.J. (2003b). Production of reactive oxygen species by mitochondria: central role of complex III. J Biol Chem 278, 36027-36031.

Chinta, S.J., Mallajosyula, J.K., Rane, A., and Andersen, J.K. (2010). Mitochondrial alpha-synuclein accumulation impairs complex I function in dopaminergic neurons and results in increased mitophagy in vivo. Neurosci Lett 486, 235-239.

Cho, D.H., Nakamura, T., and Lipton, S.A. (2010). Mitochondrial dynamics in cell death and neurodegeneration. Cell Mol Life Sci 67, 3435-3447.

Choi, W., Zibaee, S., Jakes, R., Serpell, L.C., Davletov, B., Crowther, R.A., and Goedert, M. (2004). Mutation E46K increases phospholipid binding and assembly into filaments of human alpha-synuclein. FEBS Lett 576, 363-368. 
Choubey, V., Safiulina, D., Vaarmann, A., Cagalinec, M., Wareski, P., Kuum, M., Zharkovsky, A., and Kaasik, A. (2011). Mutant A53T alpha-synuclein induces neuronal death by increasing mitochondrial autophagy. J Biol Chem 286, 10814-10824.

Cipolat, S., Martins de Brito, O., Dal Zilio, B., and Scorrano, L. (2004). OPA1 requires mitofusin 1 to promote mitochondrial fusion. Proc Natl Acad Sci U S A 101, 15927-15932.

Clark, I.E., Dodson, M.W., Jiang, C., Cao, J.H., Huh, J.R., Seol, J.H., Yoo, S.J., Hay, B.A., and Guo, M. (2006). Drosophila pink1 is required for mitochondrial function and interacts genetically with parkin. Nature 441, 1162-1166.

Clark, S.G., Stern, M.J., and Horvitz, H.R. (1992). Genes involved in two Caenorhabditis elegans cellsignaling pathways. Cold Spring Harb Symp Quant Biol 57, 363-373.

Conway, K.A., Harper, J.D., and Lansbury, P.T. (1998). Accelerated in vitro fibril formation by a mutant alpha-synuclein linked to early-onset Parkinson disease. Nat Med 4, 1318-1320.

Conway, K.A., Lee, S.J., Rochet, J.C., Ding, T.T., Harper, J.D., Williamson, R.E., and Lansbury, P.T., Jr. (2000). Accelerated oligomerization by Parkinson's disease linked alpha-synuclein mutants. Ann N Y Acad Sci 920, 42-45.

Conway, K.A., Rochet, J.C., Bieganski, R.M., and Lansbury, P.T., Jr. (2001). Kinetic stabilization of the alpha-synuclein protofibril by a dopamine-alpha-synuclein adduct. Science 294, 1346-1349.

Cooper, A.A., Gitler, A.D., Cashikar, A., Haynes, C.M., Hill, K.J., Bhullar, B., Liu, K., Xu, K., Strathearn, K.E., Liu, F., et al. (2006). Alpha-synuclein blocks ER-Golgi traffic and Rab1 rescues neuron loss in Parkinson's models. Science 313, 324-328.

Corti, O., Hampe, C., Koutnikova, H., Darios, F., Jacquier, S., Prigent, A., Robinson, J.C., Pradier, L., Ruberg, M., Mirande, M., et al. (2003). The p38 subunit of the aminoacyl-tRNA synthetase complex is a Parkin substrate: linking protein biosynthesis and neurodegeneration. Hum Mol Genet 12, 14271437.

Corti, O., Lesage, S., and Brice, A. (2011). What genetics tells us about the causes and mechanisms of Parkinson's disease. Physiol Rev 91, 1161-1218.

Cribbs, J.T., and Strack, S. (2007). Reversible phosphorylation of Drp1 by cyclic AMP-dependent protein kinase and calcineurin regulates mitochondrial fission and cell death. EMBO Rep 8, 939-944.

Cuervo, A.M., Stefanis, L., Fredenburg, R., Lansbury, P.T., and Sulzer, D. (2004). Impaired degradation of mutant alpha-synuclein by chaperone-mediated autophagy. Science 305, 1292-1295.

Cunningham, J.T., Rodgers, J.T., Arlow, D.H., Vazquez, F., Mootha, V.K., and Puigserver, P. (2007). mTOR controls mitochondrial oxidative function through a YY1-PGC-1alpha transcriptional complex. Nature 450, 736-740.

de Rijk, M.C., Tzourio, C., Breteler, M.M., Dartigues, J.F., Amaducci, L., Lopez-Pousa, S., ManubensBertran, J.M., Alperovitch, A., and Rocca, W.A. (1997). Prevalence of parkinsonism and Parkinson's disease in Europe: the EUROPARKINSON Collaborative Study. European Community Concerted Action on the Epidemiology of Parkinson's disease. J Neurol Neurosurg Psychiatry 62, 10-15.

Delettre, C., Lenaers, G., Griffoin, J.M., Gigarel, N., Lorenzo, C., Belenguer, P., Pelloquin, L., Grosgeorge, J., Turc-Carel, C., Perret, E., et al. (2000). Nuclear gene OPA1, encoding a mitochondrial dynamin-related protein, is mutated in dominant optic atrophy. Nat Genet 26, 207-210.

Deng, H., Dodson, M.W., Huang, H., and Guo, M. (2008). The Parkinson's disease genes pink1 and parkin promote mitochondrial fission and/or inhibit fusion in Drosophila. Proc Natl Acad Sci U S A 105, 14503-14508.

Detmer, S.A., and Chan, D.C. (2007). Functions and dysfunctions of mitochondrial dynamics. Nat Rev Mol Cell Biol 8, 870-879. 
Devi, L., Raghavendran, V., Prabhu, B.M., Avadhani, N.G., and Anandatheerthavarada, H.K. (2008). Mitochondrial import and accumulation of alpha-synuclein impair complex I in human dopaminergic neuronal cultures and Parkinson disease brain. J Biol Chem 283, 9089-9100.

Dexter, D.T., Carayon, A., Javoy-Agid, F., Agid, Y., Wells, F.R., Daniel, S.E., Lees, A.J., Jenner, P., and Marsden, C.D. (1991). Alterations in the levels of iron, ferritin and other trace metals in Parkinson's disease and other neurodegenerative diseases affecting the basal ganglia. Brain 114 ( Pt 4), 1953-1975.

Di Fonzo, A., Chien, H.F., Socal, M., Giraudo, S., Tassorelli, C., Iliceto, G., Fabbrini, G., Marconi, R., Fincati, E., Abbruzzese, G., et al. (2007). ATP13A2 missense mutations in juvenile parkinsonism and young onset Parkinson disease. Neurology 68, 1557-1562.

Di Fonzo, A., Rohe, C.F., Ferreira, J., Chien, H.F., Vacca, L., Stocchi, F., Guedes, L., Fabrizio, E., Manfredi, M., Vanacore, N., et al. (2005). A frequent LRRK2 gene mutation associated with autosomal dominant Parkinson's disease. Lancet 365, 412-415.

Dillin, A., Hsu, A.L., Arantes-Oliveira, N., Lehrer-Graiwer, J., Hsin, H., Fraser, A.G., Kamath, R.S., Ahringer, J., and Kenyon, C. (2002). Rates of behavior and aging specified by mitochondrial function during development. Science 298, 2398-2401.

Dimmer, K.S., and Scorrano, L. (2006). (De)constructing mitochondria: what for? Physiology (Bethesda) 21, 233-241.

Djarmati, A., Hagenah, J., Reetz, K., Winkler, S., Behrens, M.I., Pawlack, H., Lohmann, K., Ramirez, A., Tadic, V., Bruggemann, N., et al. (2009). ATP13A2 variants in early-onset Parkinson's disease patients and controls. Mov Disord 24, 2104-2111.

Djarmati, A., Hedrich, K., Svetel, M., Lohnau, T., Schwinger, E., Romac, S., Pramstaller, P.P., Kostic, V., and Klein, C. (2006). Heterozygous PINK1 mutations: a susceptibility factor for Parkinson disease? Mov Disord 21, 1526-1530.

Dooley, C.T., Dore, T.M., Hanson, G.T., Jackson, W.C., Remington, S.J., and Tsien, R.Y. (2004). Imaging dynamic redox changes in mammalian cells with green fluorescent protein indicators. J Biol Chem 279, 22284-22293.

Double, K.L., Ben-Shachar, D., Youdim, M.B., Zecca, L., Riederer, P., and Gerlach, M. (2002). Influence of neuromelanin on oxidative pathways within the human substantia nigra. Neurotoxicol Teratol 24, 621-628.

Durieux, J., Wolff, S., and Dillin, A. (2011). The cell-non-autonomous nature of electron transport chain-mediated longevity. Cell 144, 79-91.

Ebrahimi-Fakhari, D., Cantuti-Castelvetri, I., Fan, Z., Rockenstein, E., Masliah, E., Hyman, B.T., McLean, P.J., and Unni, V.K. (2011). Distinct roles in vivo for the ubiquitin-proteasome system and the autophagy-lysosomal pathway in the degradation of alpha-synuclein. J Neurosci 31, 14508-14520.

Egan, D., Kim, J., Shaw, R.J., and Guan, K.L. (2011a). The autophagy initiating kinase ULK1 is regulated via opposing phosphorylation by AMPK and mTOR. Autophagy 7, 643-644.

Egan, D.F., Shackelford, D.B., Mihaylova, M.M., Gelino, S., Kohnz, R.A., Mair, W., Vasquez, D.S., Joshi, A., Gwinn, D.M., Taylor, R., et al. (2011b). Phosphorylation of ULK1 (hATG1) by AMP-activated protein kinase connects energy sensing to mitophagy. Science 331, 456-461.

Eimer, S., Donhauser, R., and Baumeister, R. (2002). The Caenorhabditis elegans presenilin sel-12 is required for mesodermal patterning and muscle function. Dev Biol 251, 178-192.

Elbaz, A., Levecque, C., Clavel, J., Vidal, J.S., Richard, F., Correze, J.R., Delemotte, B., Amouyel, P., Alperovitch, A., Chartier-Harlin, M.C., et al. (2003). S18Y polymorphism in the UCH-L1 gene and Parkinson's disease: evidence for an age-dependent relationship. Mov Disord 18, 130-137. 
Eliezer, D., Kutluay, E., Bussell, R., Jr., and Browne, G. (2001). Conformational properties of alphasynuclein in its free and lipid-associated states. J Mol Biol 307, 1061-1073.

Enochs, W.S., Sarna, T., Zecca, L., Riley, P.A., and Swartz, H.M. (1994). The roles of neuromelanin, binding of metal ions, and oxidative cytotoxicity in the pathogenesis of Parkinson's disease: a hypothesis. J Neural Transm Park Dis Dement Sect 7, 83-100.

Facheris, M., Strain, K.J., Lesnick, T.G., de Andrade, M., Bower, J.H., Ahlskog, J.E., Cunningham, J.M., Lincoln, S., Farrer, M.J., Rocca, W.A., et al. (2005). UCHL1 is associated with Parkinson's disease: a case-unaffected sibling and case-unrelated control study. Neurosci Lett 381, 131-134.

Fahn, S. (2003). Description of Parkinson's disease as a clinical syndrome. Ann N Y Acad Sci 991, 114.

Farrer, M., Kachergus, J., Forno, L., Lincoln, S., Wang, D.S., Hulihan, M., Maraganore, D., GwinnHardy, K., Wszolek, Z., Dickson, D., et al. (2004). Comparison of kindreds with parkinsonism and alpha-synuclein genomic multiplications. Ann Neurol 55, 174-179.

Finkel, T. (2011). Signal transduction by reactive oxygen species. J Cell Biol 194, 7-15.

Fong, C.Y., Rolfs, A., Schwarzbraun, T., Klein, C., and O'Callaghan, F.J. (2011). Juvenile parkinsonism associated with heterozygous frameshift ATP13A2 gene mutation. Eur J Paediatr Neurol $15,271-275$.

Forno, L.S. (1996). Neuropathology of Parkinson's disease. J Neuropathol Exp Neurol 55, 259-272.

Fuchs, J., Tichopad, A., Golub, Y., Munz, M., Schweitzer, K.J., Wolf, B., Berg, D., Mueller, J.C., and Gasser, T. (2008). Genetic variability in the SNCA gene influences alpha-synuclein levels in the blood and brain. FASEB J 22, 1327-1334.

Fukuda, T., Ewan, L., Bauer, M., Mattaliano, R.J., Zaal, K., Ralston, E., Plotz, P.H., and Raben, N. (2006). Dysfunction of endocytic and autophagic pathways in a lysosomal storage disease. Ann Neurol 59, 700-708.

Funayama, M., Hasegawa, K., Kowa, H., Saito, M., Tsuji, S., and Obata, F. (2002). A new locus for Parkinson's disease (PARK8) maps to chromosome 12p11.2-q13.1. Ann Neurol 51, 296-301.

Gartner, A., Boag, P.R., and Blackwell, T.K. (2008). Germline survival and apoptosis. WormBook, 120.

Gegg, M.E., and Schapira, A.H. (2011). PINK1-parkin-dependent mitophagy involves ubiquitination of mitofusins 1 and 2: Implications for Parkinson disease pathogenesis. Autophagy 7, 243-245.

Geisler, S., Holmstrom, K.M., Skujat, D., Fiesel, F.C., Rothfuss, O.C., Kahle, P.J., and Springer, W. (2010). PINK1/Parkin-mediated mitophagy is dependent on VDAC1 and p62/SQSTM1. Nat Cell Biol $12,119-131$.

Giasson, B.I., Murray, I.V., Trojanowski, J.Q., and Lee, V.M. (2001). A hydrophobic stretch of 12 amino acid residues in the middle of alpha-synuclein is essential for filament assembly. $J$ Biol Chem $276,2380-2386$.

Giasson, B.I., Uryu, K., Trojanowski, J.Q., and Lee, V.M. (1999). Mutant and wild type human alphasynucleins assemble into elongated filaments with distinct morphologies in vitro. J Biol Chem 274, 7619-7622.

Gilks, W.P., Abou-Sleiman, P.M., Gandhi, S., Jain, S., Singleton, A., Lees, A.J., Shaw, K., Bhatia, K.P., Bonifati, V., Quinn, N.P., et al. (2005). A common LRRK2 mutation in idiopathic Parkinson's disease. Lancet 365, 415-416.

Gitler, A.D., Chesi, A., Geddie, M.L., Strathearn, K.E., Hamamichi, S., Hill, K.J., Caldwell, K.A., Caldwell, G.A., Cooper, A.A., Rochet, J.C., et al. (2009). Alpha-synuclein is part of a diverse and 
highly conserved interaction network that includes PARK9 and manganese toxicity. Nat Genet 41, 308-315.

Glabe, C.G. (2006). Common mechanisms of amyloid oligomer pathogenesis in degenerative disease. Neurobiol Aging 27, 570-575.

Gloeckner, C.J., Kinkl, N., Schumacher, A., Braun, R.J., O'Neill, E., Meitinger, T., Kolch, W., Prokisch, H., and Ueffing, M. (2006). The Parkinson disease causing LRRK2 mutation I2020T is associated with increased kinase activity. Hum Mol Genet 15, 223-232.

Goldberg, M.S., and Lansbury, P.T., Jr. (2000). Is there a cause-and-effect relationship between alpha-synuclein fibrillization and Parkinson's disease? Nat Cell Biol 2, E115-119.

Gomes, L.C., Di Benedetto, G., and Scorrano, L. (2011). During autophagy mitochondria elongate, are spared from degradation and sustain cell viability. Nat Cell Biol 13, 589-598.

Gosavi, N., Lee, H.J., Lee, J.S., Patel, S., and Lee, S.J. (2002). Golgi fragmentation occurs in the cells with prefibrillar alpha-synuclein aggregates and precedes the formation of fibrillar inclusion. J Biol Chem 277, 48984-48992.

Gray, M.W., and Doolittle, W.F. (1982). Has the endosymbiont hypothesis been proven? Microbiol Rev 46, 1-42.

Greenbaum, E.A., Graves, C.L., Mishizen-Eberz, A.J., Lupoli, M.A., Lynch, D.R., Englander, S.W., Axelsen, P.H., and Giasson, B.I. (2005). The E46K mutation in alpha-synuclein increases amyloid fibril formation. J Biol Chem 280, 7800-7807.

Greene, J.C., Whitworth, A.J., Kuo, I., Andrews, L.A., Feany, M.B., and Pallanck, L.J. (2003). Mitochondrial pathology and apoptotic muscle degeneration in Drosophila parkin mutants. Proc Natl Acad Sci U S A 100, 4078-4083.

Greenwald, I.S., Sternberg, P.W., and Horvitz, H.R. (1983). The lin-12 locus specifies cell fates in Caenorhabditis elegans. Cell 34, 435-444.

Greggio, E., Zambrano, I., Kaganovich, A., Beilina, A., Taymans, J.M., Daniels, V., Lewis, P., Jain, S., Ding, J., Syed, A., et al. (2008). The Parkinson disease-associated leucine-rich repeat kinase 2 (LRRK2) is a dimer that undergoes intramolecular autophosphorylation. J Biol Chem 283, 1690616914.

Griffin, E.E., Detmer, S.A., and Chan, D.C. (2006). Molecular mechanism of mitochondrial membrane fusion. Biochim Biophys Acta 1763, 482-489.

Grunewald, A., Arns, B., Seibler, P., Rakovic, A., Munchau, A., Ramirez, A., Sue, C.M., and Klein, C. (2012). ATP13A2 mutations impair mitochondrial function in fibroblasts from patients with Kufor-Rakeb syndrome. Neurobiol Aging.

Guo, L., Gandhi, P.N., Wang, W., Petersen, R.B., Wilson-Delfosse, A.L., and Chen, S.G. (2007). The Parkinson's disease-associated protein, leucine-rich repeat kinase 2 (LRRK2), is an authentic GTPase that stimulates kinase activity. Exp Cell Res 313, 3658-3670.

Gusdon, A.M., Zhu, J., Van Houten, B., and Chu, C.T. (2011). ATP13A2 regulates mitochondrial bioenergetics through macroautophagy. Neurobiol Dis.

Guzman, J.N., Sanchez-Padilla, J., Wokosin, D., Kondapalli, J., Ilijic, E., Schumacker, P.T., and Surmeier, D.J. (2010). Oxidant stress evoked by pacemaking in dopaminergic neurons is attenuated by DJ-1. Nature 468, 696-700.

Haas, R.H., Nasirian, F., Nakano, K., Ward, D., Pay, M., Hill, R., and Shults, C.W. (1995). Low platelet mitochondrial complex I and complex II/III activity in early untreated Parkinson's disease. Ann Neurol 37, 714-722. 
Hales, K.G., and Fuller, M.T. (1997). Developmentally regulated mitochondrial fusion mediated by a conserved, novel, predicted GTPase. Cell 90, 121-129.

Hamamichi, S., Rivas, R.N., Knight, A.L., Cao, S., Caldwell, K.A., and Caldwell, G.A. (2008). Hypothesis-based RNAi screening identifies neuroprotective genes in a Parkinson's disease model. Proc Natl Acad Sci U S A 105, 728-733.

Hardie, D.G. (2004). The AMP-activated protein kinase pathway--new players upstream and downstream. J Cell Sci 117, 5479-5487.

Hardie, D.G. (2011). AMP-activated protein kinase: an energy sensor that regulates all aspects of cell function. Genes Dev 25, 1895-1908.

Harfe, B.D., Branda, C.S., Krause, M., Stern, M.J., and Fire, A. (1998). MyoD and the specification of muscle and non-muscle fates during postembryonic development of the C. elegans mesoderm. Development 125, 2479-2488.

Harman, D. (1956). Aging: a theory based on free radical and radiation chemistry. J Gerontol 11, 298300.

Hartman, P.S., Ishii, N., Kayser, E.B., Morgan, P.G., and Sedensky, M.M. (2001). Mitochondrial mutations differentially affect aging, mutability and anesthetic sensitivity in Caenorhabditis elegans. Mech Ageing Dev 122, 1187-1201.

Hatano, T., Kubo, S., Imai, S., Maeda, M., Ishikawa, K., Mizuno, Y., and Hattori, N. (2007). Leucinerich repeat kinase 2 associates with lipid rafts. Hum Mol Genet 16, 678-690.

Hawley, S.A., Davison, M., Woods, A., Davies, S.P., Beri, R.K., Carling, D., and Hardie, D.G. (1996). Characterization of the AMP-activated protein kinase kinase from rat liver and identification of threonine 172 as the major site at which it phosphorylates AMP-activated protein kinase. J Biol Chem $271,27879-27887$.

Haynes, C.M., Petrova, K., Benedetti, C., Yang, Y., and Ron, D. (2007). ClpP mediates activation of a mitochondrial unfolded protein response in C. elegans. Dev Cell 13, 467-480.

Haynes, C.M., Yang, Y., Blais, S.P., Neubert, T.A., and Ron, D. (2010). The matrix peptide exporter HAF-1 signals a mitochondrial UPR by activating the transcription factor ZC376.7 in C. elegans. Mol Cell 37, 529-540.

Haywood, A.F., and Staveley, B.E. (2006). Mutant alpha-synuclein-induced degeneration is reduced by parkin in a fly model of Parkinson's disease. Genome 49, 505-510.

Head, B.P., Zulaika, M., Ryazantsev, S., and van der Bliek, A.M. (2011). A novel mitochondrial outer membrane protein, MOMA-1, that affects cristae morphology in Caenorhabditis elegans. Mol Biol Cell 22, 831-841.

Heise, H., Hoyer, W., Becker, S., Andronesi, O.C., Riedel, D., and Baldus, M. (2005). Molecular-level secondary structure, polymorphism, and dynamics of full-length alpha-synuclein fibrils studied by solidstate NMR. Proc Natl Acad Sci U S A 102, 15871-15876.

Henchcliffe, C., and Beal, M.F. (2008). Mitochondrial biology and oxidative stress in Parkinson disease pathogenesis. Nat Clin Pract Neurol 4, 600-609.

Hill, R.J., and Sternberg, P.W. (1992). The gene lin-3 encodes an inductive signal for vulval development in C. elegans. Nature 358, 470-476.

Hirsh, D., Oppenheim, D., and Klass, M. (1976). Development of the reproductive system of Caenorhabditis elegans. Dev Biol 49, 200-219.

Hoppins, S., Lackner, L., and Nunnari, J. (2007). The machines that divide and fuse mitochondria. Annu Rev Biochem 76, 751-780. 
Hsu, L.J., Sagara, Y., Arroyo, A., Rockenstein, E., Sisk, A., Mallory, M., Wong, J., Takenouchi, T., Hashimoto, M., and Masliah, E. (2000). alpha-synuclein promotes mitochondrial deficit and oxidative stress. Am J Pathol 157, 401-410.

Huang, L., and Hanna-Rose, W. (2006). EGF signaling overcomes a uterine cell death associated with temporal mis-coordination of organogenesis within the C. elegans egg-laying apparatus. Dev Biol 300, 599-611.

Hudson, S.A., Ecroyd, H., Kee, T.W., and Carver, J.A. (2009). The thioflavin T fluorescence assay for amyloid fibril detection can be biased by the presence of exogenous compounds. FEBS J 276, 59605972.

Ibanez, P., Bonnet, A.M., Debarges, B., Lohmann, E., Tison, F., Pollak, P., Agid, Y., Durr, A., and Brice, A. (2004). Causal relation between alpha-synuclein gene duplication and familial Parkinson's disease. Lancet 364, 1169-1171.

Imai, Y., and Lu, B. (2011). Mitochondrial dynamics and mitophagy in Parkinson's disease: disordered cellular power plant becomes a big deal in a major movement disorder. Curr Opin Neurobiol 21, 935941.

Ishii, N., Fujii, M., Hartman, P.S., Tsuda, M., Yasuda, K., Senoo-Matsuda, N., Yanase, S., Ayusawa, D., and Suzuki, K. (1998). A mutation in succinate dehydrogenase cytochrome b causes oxidative stress and ageing in nematodes. Nature 394, 694-697.

Ishii, N., Senoo-Matsuda, N., Miyake, K., Yasuda, K., Ishii, T., Hartman, P.S., and Furukawa, S. (2004). Coenzyme Q10 can prolong C. elegans lifespan by lowering oxidative stress. Mech Ageing Dev 125, 41-46.

Ishii, N., Takahashi, K., Tomita, S., Keino, T., Honda, S., Yoshino, K., and Suzuki, K. (1990). A methyl viologen-sensitive mutant of the nematode Caenorhabditis elegans. Mutat Res 237, 165-171.

Jennings, J.J., Jr., Zhu, J.H., Rbaibi, Y., Luo, X., Chu, C.T., and Kiselyov, K. (2006). Mitochondrial aberrations in mucolipidosis Type IV. J Biol Chem 281, 39041-39050.

Jung, C.H., Ro, S.H., Cao, J., Otto, N.M., and Kim, D.H. (2010). mTOR regulation of autophagy. FEBS Lett 584, 1287-1295.

Kahle, P.J., Neumann, M., Ozmen, L., Muller, V., Jacobsen, H., Schindzielorz, A., Okochi, M., Leimer, U., van Der Putten, H., Probst, A., et al. (2000). Subcellular localization of wild-type and Parkinson's disease-associated mutant alpha -synuclein in human and transgenic mouse brain. J Neurosci 20 , 6365-6373.

Kamath, R.S., and Ahringer, J. (2003). Genome-wide RNAi screening in Caenorhabditis elegans. Methods 30, 313-321.

Kamp, F., Exner, N., Lutz, A.K., Wender, N., Hegermann, J., Brunner, B., Nuscher, B., Bartels, T., Giese, A., Beyer, K., et al. (2010). Inhibition of mitochondrial fusion by alpha-synuclein is rescued by PINK1, Parkin and DJ-1. EMBO J 29, 3571-3589.

Kanazawa, T., Zappaterra, M.D., Hasegawa, A., Wright, A.P., Newman-Smith, E.D., Buttle, K.F., McDonald, K., Mannella, C.A., and van der Bliek, A.M. (2008). The C. elegans Opa1 homologue EAT3 is essential for resistance to free radicals. PLoS Genet 4, e1000022.

Kang, C., You, Y.J., and Avery, L. (2007). Dual roles of autophagy in the survival of Caenorhabditis elegans during starvation. Genes Dev 21, 2161-2171.

Kanki, T., and Klionsky, D.J. (2010). The molecular mechanism of mitochondria autophagy in yeast. Mol Microbiol 75, 795-800.

Karpinar, D.P., Balija, M.B., Kugler, S., Opazo, F., Rezaei-Ghaleh, N., Wender, N., Kim, H.Y., Taschenberger, G., Falkenburger, B.H., Heise, H., et al. (2009). Pre-fibrillar alpha-synuclein variants 
with impaired beta-structure increase neurotoxicity in Parkinson's disease models. EMBO J 28, 32563268.

Katz, W.S., Hill, R.J., Clandinin, T.R., and Sternberg, P.W. (1995). Different levels of the C. elegans growth factor LIN-3 promote distinct vulval precursor fates. Cell 82, 297-307.

Kayser, E.B., Morgan, P.G., and Sedensky, M.M. (1999). GAS-1: a mitochondrial protein controls sensitivity to volatile anesthetics in the nematode Caenorhabditis elegans. Anesthesiology $90,545-$ 554.

Kayser, E.B., Sedensky, M.M., and Morgan, P.G. (2004). The effects of complex I function and oxidative damage on lifespan and anesthetic sensitivity in Caenorhabditis elegans. Mech Ageing Dev $125,455-464$.

Kim, J., Kundu, M., Viollet, B., and Guan, K.L. (2011). AMPK and mTOR regulate autophagy through direct phosphorylation of Ulk1. Nat Cell Biol 13, 132-141.

Kimble, J. (1981). Alterations in cell lineage following laser ablation of cells in the somatic gonad of Caenorhabditis elegans. Dev Biol 87, 286-300.

Kitada, T., Asakawa, S., Hattori, N., Matsumine, H., Yamamura, Y., Minoshima, S., Yokochi, M., Mizuno, Y., and Shimizu, N. (1998). Mutations in the parkin gene cause autosomal recessive juvenile parkinsonism. Nature 392, 605-608.

Knott, A.B., Perkins, G., Schwarzenbacher, R., and Bossy-Wetzel, E. (2008). Mitochondrial fragmentation in neurodegeneration. Nat Rev Neurosci 9, 505-518.

Kondo, M., Senoo-Matsuda, N., Yanase, S., Ishii, T., Hartman, P.S., and Ishii, N. (2005). Effect of oxidative stress on translocation of DAF-16 in oxygen-sensitive mutants, mev-1 and gas-1 of Caenorhabditis elegans. Mech Ageing Dev 126, 637-641.

Korolchuk, V.I., Saiki, S., Lichtenberg, M., Siddiqi, F.H., Roberts, E.A., Imarisio, S., Jahreiss, L., Sarkar, S., Futter, M., Menzies, F.M., et al. (2011). Lysosomal positioning coordinates cellular nutrient responses. Nat Cell Biol 13, 453-460.

Koyanagi, M., Asahara, S., Matsuda, T., Hashimoto, N., Shigeyama, Y., Shibutani, Y., Kanno, A., Fuchita, M., Mikami, T., Hosooka, T., et al. (2011). Ablation of TSC2 enhances insulin secretion by increasing the number of mitochondria through activation of mTORC1. PLoS One 6, e23238.

Krige, D., Carroll, M.T., Cooper, J.M., Marsden, C.D., and Schapira, A.H. (1992). Platelet mitochondrial function in Parkinson's disease. The Royal Kings and Queens Parkinson Disease Research Group. Ann Neurol 32, 782-788.

Kruger, R., Kuhn, W., Muller, T., Woitalla, D., Graeber, M., Kosel, S., Przuntek, H., Epplen, J.T., Schols, L., and Riess, O. (1998). Ala30Pro mutation in the gene encoding alpha-synuclein in Parkinson's disease. Nat Genet 18, 106-108.

Kubo, S., Nemani, V.M., Chalkley, R.J., Anthony, M.D., Hattori, N., Mizuno, Y., Edwards, R.H., and Fortin, D.L. (2005). A combinatorial code for the interaction of alpha-synuclein with membranes. J Biol Chem 280, 31664-31672.

Kudin, A.P., Bimpong-Buta, N.Y., Vielhaber, S., Elger, C.E., and Kunz, W.S. (2004). Characterization of superoxide-producing sites in isolated brain mitochondria. J Biol Chem 279, 4127-4135.

Kuhlbrandt, W. (2004). Biology, structure and mechanism of P-type ATPases. Nat Rev Mol Cell Biol 5, 282-295.

Kumar, A., and Cookson, M.R. (2011). Role of LRRK2 kinase dysfunction in Parkinson disease. Expert Rev Mol Med 13, e20. 
Kuwahara, T., Koyama, A., Gengyo-Ando, K., Masuda, M., Kowa, H., Tsunoda, M., Mitani, S., and Iwatsubo, T. (2006). Familial Parkinson mutant alpha-synuclein causes dopamine neuron dysfunction in transgenic Caenorhabditis elegans. J Biol Chem 281, 334-340.

Kuznetsov, A.V., Hermann, M., Saks, V., Hengster, P., and Margreiter, R. (2009). The cell-type specificity of mitochondrial dynamics. Int J Biochem Cell Biol 41, 1928-1939.

Labrousse, A.M., Zappaterra, M.D., Rube, D.A., and van der Bliek, A.M. (1999). C. elegans dynaminrelated protein DRP-1 controls severing of the mitochondrial outer membrane. Mol Cell 4, 815-826.

Lackner, L.L., and Nunnari, J.M. (2009). The molecular mechanism and cellular functions of mitochondrial division. Biochim Biophys Acta 1792, 1138-1144.

Lakso, M., Vartiainen, S., Moilanen, A.M., Sirvio, J., Thomas, J.H., Nass, R., Blakely, R.D., and Wong, G. (2003). Dopaminergic neuronal loss and motor deficits in Caenorhabditis elegans overexpressing human alpha-synuclein. J Neurochem 86, 165-172.

Larsen, C.N., Krantz, B.A., and Wilkinson, K.D. (1998). Substrate specificity of deubiquitinating enzymes: ubiquitin C-terminal hydrolases. Biochemistry 37, 3358-3368.

Lee, H., Cho, J.S., Lambacher, N., Lee, J., Lee, S.J., Lee, T.H., Gartner, A., and Koo, H.S. (2008). The Caenorhabditis elegans AMP-activated protein kinase AAK-2 is phosphorylated by LKB1 and is required for resistance to oxidative stress and for normal motility and foraging behavior. $\mathrm{J}$ Biol Chem 283, 14988-14993.

Lee, H.J., Khoshaghideh, F., Lee, S., and Lee, S.J. (2006). Impairment of microtubule-dependent trafficking by overexpression of alpha-synuclein. Eur J Neurosci 24, 3153-3162.

Lee, M., Hyun, D., Halliwell, B., and Jenner, P. (2001). Effect of the overexpression of wild-type or mutant alpha-synuclein on cell susceptibility to insult. J Neurochem 76, 998-1009.

Lee, M.K., Stirling, W., Xu, Y., Xu, X., Qui, D., Mandir, A.S., Dawson, T.M., Copeland, N.G., Jenkins, N.A., and Price, D.L. (2002). Human alpha-synuclein-harboring familial Parkinson's disease-linked Ala-53 --> Thr mutation causes neurodegenerative disease with alpha-synuclein aggregation in transgenic mice. Proc Natl Acad Sci U S A 99, 8968-8973.

Lees, A.J., and Singleton, A.B. (2007). Clinical heterogeneity of ATP13A2 linked disease (KuforRakeb) justifies a PARK designation. Neurology 68, 1553-1554.

Leroy, E., Boyer, R., Auburger, G., Leube, B., Ulm, G., Mezey, E., Harta, G., Brownstein, M.J., Jonnalagada, S., Chernova, T., et al. (1998). The ubiquitin pathway in Parkinson's disease. Nature 395, 451-452.

Lesage, S., Janin, S., Lohmann, E., Leutenegger, A.L., Leclere, L., Viallet, F., Pollak, P., Durif, F., Thobois, S., Layet, V., et al. (2007). LRRK2 exon 41 mutations in sporadic Parkinson disease in Europeans. Arch Neurol 64, 425-430.

Lewy, F.H. (1912). Paralysis agitans - I. Pathologische Anatomie. In Handbuch der Neurologie, M. Lewandowski, ed. (Springer Verlag, Berlin), pp. 920-933.

Li, J., Uversky, V.N., and Fink, A.L. (2001). Effect of familial Parkinson's disease point mutations A30P and $\mathrm{A} 53 \mathrm{~T}$ on the structural properties, aggregation, and fibrillation of human alpha-synuclein. Biochemistry 40, 11604-11613.

Li, W.W., Yang, R., Guo, J.C., Ren, H.M., Zha, X.L., Cheng, J.S., and Cai, D.F. (2007). Localization of alpha-synuclein to mitochondria within midbrain of mice. Neuroreport 18, 1543-1546.

Lin, C.H., Tan, E.K., Chen, M.L., Tan, L.C., Lim, H.Q., Chen, G.S., and Wu, R.M. (2008). Novel ATP13A2 variant associated with Parkinson disease in Taiwan and Singapore. Neurology 71, 17271732. 
Link, C.D., Cypser, J.R., Johnson, C.J., and Johnson, T.E. (1999). Direct observation of stress response in Caenorhabditis elegans using a reporter transgene. Cell Stress Chaperones 4, 235-242.

Locke, C.J., Fox, S.A., Caldwell, G.A., and Caldwell, K.A. (2008). Acetaminophen attenuates dopamine neuron degeneration in animal models of Parkinson's disease. Neurosci Lett 439, 129-133.

Lotharius, J., and Brundin, P. (2002). Pathogenesis of Parkinson's disease: dopamine, vesicles and alpha-synuclein. Nat Rev Neurosci 3, 932-942.

MacLeod, D., Dowman, J., Hammond, R., Leete, T., Inoue, K., and Abeliovich, A. (2006). The familial Parkinsonism gene LRRK2 regulates neurite process morphology. Neuron 52, 587-593.

Marin, I., van Egmond, W.N., and van Haastert, P.J. (2008). The Roco protein family: a functional perspective. FASEB J 22, 3103-3110.

Martin, L.J., Pan, Y., Price, A.C., Sterling, W., Copeland, N.G., Jenkins, N.A., Price, D.L., and Lee, M.K. (2006). Parkinson's disease alpha-synuclein transgenic mice develop neuronal mitochondrial degeneration and cell death. J Neurosci 26, 41-50.

Martinez-Vicente, M., Talloczy, Z., Kaushik, S., Massey, A.C., Mazzulli, J., Mosharov, E.V., Hodara, R., Fredenburg, R., Wu, D.C., Follenzi, A., et al. (2008). Dopamine-modified alpha-synuclein blocks chaperone-mediated autophagy. J Clin Invest 118, 777-788.

Maruyama, W., Shamoto-Nagai, M., Akao, Y., Riederer, P., and Naoi, M. (2006). The effect of neuromelanin on the proteasome activity in human dopaminergic SH-SY5Y cells. J Neural Transm Suppl, 125-132.

Masliah, E., Rockenstein, E., Veinbergs, I., Mallory, M., Hashimoto, M., Takeda, A., Sagara, Y., Sisk, A., and Mucke, L. (2000). Dopaminergic loss and inclusion body formation in alpha-synuclein mice: implications for neurodegenerative disorders. Science 287, 1265-1269.

Mata, I.F., Lockhart, P.J., and Farrer, M.J. (2004). Parkin genetics: one model for Parkinson's disease. Hum Mol Genet 13 Spec No 1, R127-133.

Mata, I.F., Wedemeyer, W.J., Farrer, M.J., Taylor, J.P., and Gallo, K.A. (2006). LRRK2 in Parkinson's disease: protein domains and functional insights. Trends Neurosci 29, 286-293.

Matsuda, N., Sato, S., Shiba, K., Okatsu, K., Saisho, K., Gautier, C.A., Sou, Y.S., Saiki, S., Kawajiri, S., Sato, F., et al. (2010). PINK1 stabilized by mitochondrial depolarization recruits Parkin to damaged mitochondria and activates latent Parkin for mitophagy. J Cell Biol 189, 211-221.

Matsumine, H., Saito, M., Shimoda-Matsubayashi, S., Tanaka, H., Ishikawa, A., Nakagawa-Hattori, Y., Yokochi, M., Kobayashi, T., Igarashi, S., Takano, H., et al. (1997). Localization of a gene for an autosomal recessive form of juvenile Parkinsonism to chromosome 6q25.2-27. Am J Hum Genet 60, 588-596.

McDonald, P.W., Jessen, T., Field, J.R., and Blakely, R.D. (2006). Dopamine signaling architecture in Caenorhabditis elegans. Cell Mol Neurobiol 26, 593-618.

Meeusen, S.L., and Nunnari, J. (2005). How mitochondria fuse. Curr Opin Cell Biol 17, 389-394.

Meissner, B., Warner, A., Wong, K., Dube, N., Lorch, A., McKay, S.J., Khattra, J., Rogalski, T., Somasiri, A., Chaudhry, I., et al. (2009). An integrated strategy to study muscle development and myofilament structure in Caenorhabditis elegans. PLoS Genet 5, e1000537.

Melendez, A., Hall, D.H., and Hansen, M. (2008). Monitoring the role of autophagy in C. elegans aging. Methods Enzymol 451, 493-520.

Melendez, A., Talloczy, Z., Seaman, M., Eskelinen, E.L., Hall, D.H., and Levine, B. (2003). Autophagy genes are essential for dauer development and life-span extension in C. elegans. Science 301, 13871391. 
Mello, C., and Fire, A. (1995). DNA transformation. Methods Cell Biol 48, 451-482.

Melov, S., Ravenscroft, J., Malik, S., Gill, M.S., Walker, D.W., Clayton, P.E., Wallace, D.C., Malfroy, B., Doctrow, S.R., and Lithgow, G.J. (2000). Extension of life-span with superoxide dismutase/catalase mimetics. Science 289, 1567-1569.

Meulener, M.C., Xu, K., Thomson, L., Ischiropoulos, H., and Bonini, N.M. (2006). Mutational analysis of DJ-1 in Drosophila implicates functional inactivation by oxidative damage and aging. Proc Natl Acad Sci U S A 103, 12517-12522.

Mihaylova, M.M., and Shaw, R.J. (2011). The AMPK signalling pathway coordinates cell growth, autophagy and metabolism. Nat Cell Biol 13, 1016-1023.

Mitsumoto, A., and Nakagawa, Y. (2001). DJ-1 is an indicator for endogenous reactive oxygen species elicited by endotoxin. Free Radic Res 35, 885-893.

Moller, A.B., Asp, T., Holm, P.B., and Palmgren, M.G. (2008). Phylogenetic analysis of P5 P-type ATPases, a eukaryotic lineage of secretory pathway pumps. Mol Phylogenet Evol 46, 619-634.

Moore, D.J., Zhang, L., Troncoso, J., Lee, M.K., Hattori, N., Mizuno, Y., Dawson, T.M., and Dawson, V.L. (2005). Association of DJ-1 and parkin mediated by pathogenic DJ-1 mutations and oxidative stress. Hum Mol Genet 14, 71-84.

Morgan, B., Sobotta, M.C., and Dick, T.P. (2011). Measuring EGSH and H2O2 with roGFP2-based redox probes. Free Radic Biol Med 51, 1943-1951.

Mortiboys, H., Johansen, K.K., Aasly, J.O., and Bandmann, O. (2010). Mitochondrial impairment in patients with Parkinson disease with the G2019S mutation in LRRK2. Neurology 75, 2017-2020.

Mozdy, A.D., and Shaw, J.M. (2003). A fuzzy mitochondrial fusion apparatus comes into focus. Nat Rev Mol Cell Biol 4, 468-478.

Mueller, J.C., Fuchs, J., Hofer, A., Zimprich, A., Lichtner, P., Illig, T., Berg, D., Wullner, U., Meitinger, T., and Gasser, T. (2005). Multiple regions of alpha-synuclein are associated with Parkinson's disease. Ann Neurol 57, 535-541.

Murphy, D.D., Rueter, S.M., Trojanowski, J.Q., and Lee, V.M. (2000). Synucleins are developmentally expressed, and alpha-synuclein regulates the size of the presynaptic vesicular pool in primary hippocampal neurons. J Neurosci 20, 3214-3220.

Najim al-Din, A.S., Wriekat, A., Mubaidin, A., Dasouki, M., and Hiari, M. (1994). Pallido-pyramidal degeneration, supranuclear upgaze paresis and dementia: Kufor-Rakeb syndrome. Acta Neurol Scand $89,347-352$.

Nakamura, K., Nemani, V.M., Azarbal, F., Skibinski, G., Levy, J.M., Egami, K., Munishkina, L., Zhang, J., Gardner, B., Wakabayashi, J., et al. (2011). Direct membrane association drives mitochondrial fission by the Parkinson disease-associated protein alpha-synuclein. J Biol Chem 286, 20710-20726.

Nakamura, K., Nemani, V.M., Wallender, E.K., Kaehlcke, K., Ott, M., and Edwards, R.H. (2008). Optical reporters for the conformation of alpha-synuclein reveal a specific interaction with mitochondria. J Neurosci 28, 12305-12317.

Narendra, D., Tanaka, A., Suen, D.F., and Youle, R.J. (2008). Parkin is recruited selectively to impaired mitochondria and promotes their autophagy. J Cell Biol 183, 795-803.

Narendra, D.P., Jin, S.M., Tanaka, A., Suen, D.F., Gautier, C.A., Shen, J., Cookson, M.R., and Youle, R.J. (2010). PINK1 is selectively stabilized on impaired mitochondria to activate Parkin. PLoS Biol 8 , e1000298. 
Narhi, L., Wood, S.J., Steavenson, S., Jiang, Y., Wu, G.M., Anafi, D., Kaufman, S.A., Martin, F., Sitney, K., Denis, P., et al. (1999). Both familial Parkinson's disease mutations accelerate alphasynuclein aggregation. J Biol Chem 274, 9843-9846.

Nass, R., and Blakely, R.D. (2003). The Caenorhabditis elegans dopaminergic system: opportunities for insights into dopamine transport and neurodegeneration. Annu Rev Pharmacol Toxicol 43, 521544.

Nass, R., Hall, D.H., Miller, D.M., 3rd, and Blakely, R.D. (2002). Neurotoxin-induced degeneration of dopamine neurons in Caenorhabditis elegans. Proc Natl Acad Sci U S A 99, 3264-3269.

Newman, A.P., Inoue, T., Wang, M., and Sternberg, P.W. (2000). The Caenorhabditis elegans heterochronic gene lin-29 coordinates the vulval-uterine-epidermal connections. Curr Biol 10, 14791488.

Newman, A.P., and Sternberg, P.W. (1996). Coordinated morphogenesis of epithelia during development of the Caenorhabditis elegans uterine-vulval connection. Proc Natl Acad Sci U S A 93, 9329-9333.

Newman, A.P., White, J.G., and Sternberg, P.W. (1995). The Caenorhabditis elegans lin-12 gene mediates induction of ventral uterine specialization by the anchor cell. Development 121, 263-271.

Newman, A.P., White, J.G., and Sternberg, P.W. (1996). Morphogenesis of the C. elegans hermaphrodite uterus. Development 122, 3617-3626.

Nishikawa, K., Li, H., Kawamura, R., Osaka, H., Wang, Y.L., Hara, Y., Hirokawa, T., Manago, Y., Amano, T., Noda, M., et al. (2003). Alterations of structure and hydrolase activity of parkinsonismassociated human ubiquitin carboxyl-terminal hydrolase L1 variants. Biochem Biophys Res Commun $304,176-183$

Norris, E.H., Giasson, B.I., Hodara, R., Xu, S., Trojanowski, J.Q., Ischiropoulos, H., and Lee, V.M. (2005). Reversible inhibition of alpha-synuclein fibrillization by dopaminochrome-mediated conformational alterations. J Biol Chem 280, 21212-21219.

Okatsu, K., Saisho, K., Shimanuki, M., Nakada, K., Shitara, H., Sou, Y.S., Kimura, M., Sato, S., Hattori, N., Komatsu, M., et al. (2010). p62/SQSTM1 cooperates with Parkin for perinuclear clustering of depolarized mitochondria. Genes Cells 15, 887-900.

Olanow, C.W., and McNaught, K.S. (2006). Ubiquitin-proteasome system and Parkinson's disease. Mov Disord 21, 1806-1823.

Olzmann, J.A., Brown, K., Wilkinson, K.D., Rees, H.D., Huai, Q., Ke, H., Levey, A.I., Li, L., and Chin, L.S. (2004). Familial Parkinson's disease-associated L166P mutation disrupts DJ-1 protein folding and function. J Biol Chem 279, 8506-8515.

Ostrerova, N., Petrucelli, L., Farrer, M., Mehta, N., Choi, P., Hardy, J., and Wolozin, B. (1999). alphaSynuclein shares physical and functional homology with 14-3-3 proteins. J Neurosci 19, 5782-5791.

Otera, H., and Mihara, K. (2011). Molecular mechanisms and physiologic functions of mitochondrial dynamics. J Biochem 149, 241-251.

Pals, P., Lincoln, S., Manning, J., Heckman, M., Skipper, L., Hulihan, M., Van den Broeck, M., De Pooter, T., Cras, P., Crook, J., et al. (2004). alpha-Synuclein promoter confers susceptibility to Parkinson's disease. Ann Neurol 56, 591-595.

Parihar, M.S., Parihar, A., Fujita, M., Hashimoto, M., and Ghafourifar, P. (2008). Mitochondrial association of alpha-synuclein causes oxidative stress. Cell Mol Life Sci 65, 1272-1284.

Park, J., Lee, S.B., Lee, S., Kim, Y., Song, S., Kim, S., Bae, E., Kim, J., Shong, M., Kim, J.M., et al. (2006). Mitochondrial dysfunction in Drosophila PINK1 mutants is complemented by parkin. Nature $441,1157-1161$. 
Park, J.S., Mehta, P., Cooper, A.A., Veivers, D., Heimbach, A., Stiller, B., Kubisch, C., Fung, V.S., Krainc, D., Mackay-Sim, A., et al. (2011). Pathogenic effects of novel mutations in the P-type ATPase ATP13A2 (PARK9) causing Kufor-Rakeb syndrome, a form of early-onset parkinsonism. Hum Mutat 32, 956-964.

Parker, W.D., Jr., Boyson, S.J., and Parks, J.K. (1989). Abnormalities of the electron transport chain in idiopathic Parkinson's disease. Ann Neurol 26, 719-723.

Parkinson, J. (2002). An essay on the shaking palsy. 1817. J Neuropsychiatry Clin Neurosci 14, 223236; discussion 222.

Parone, P.A., Da Cruz, S., Tondera, D., Mattenberger, Y., James, D.I., Maechler, P., Barja, F., and Martinou, J.C. (2008). Preventing mitochondrial fission impairs mitochondrial function and leads to loss of mitochondrial DNA. PLoS One 3, e3257.

Perez, R.G., Waymire, J.C., Lin, E., Liu, J.J., Guo, F., and Zigmond, M.J. (2002). A role for alphasynuclein in the regulation of dopamine biosynthesis. J Neurosci 22, 3090-3099.

Perrin, R.J., Woods, W.S., Clayton, D.F., and George, J.M. (2000). Interaction of human alphaSynuclein and Parkinson's disease variants with phospholipids. Structural analysis using site-directed mutagenesis. J Biol Chem 275, 34393-34398.

Petrucelli, L., O'Farrell, C., Lockhart, P.J., Baptista, M., Kehoe, K., Vink, L., Choi, P., Wolozin, B., Farrer, M., Hardy, J., et al. (2002). Parkin protects against the toxicity associated with mutant alphasynuclein: proteasome dysfunction selectively affects catecholaminergic neurons. Neuron $36,1007-$ 1019.

Polymeropoulos, M.H., Lavedan, C., Leroy, E., Ide, S.E., Dehejia, A., Dutra, A., Pike, B., Root, H., Rubenstein, J., Boyer, R., et al. (1997). Mutation in the alpha-synuclein gene identified in families with Parkinson's disease. Science 276, 2045-2047.

Poon, H.F., Frasier, M., Shreve, N., Calabrese, V., Wolozin, B., and Butterfield, D.A. (2005). Mitochondrial associated metabolic proteins are selectively oxidized in A30P alpha-synuclein transgenic mice--a model of familial Parkinson's disease. Neurobiol Dis 18, 492-498.

Ramirez, A., Heimbach, A., Grundemann, J., Stiller, B., Hampshire, D., Cid, L.P., Goebel, I., Mubaidin, A.F., Wriekat, A.L., Roeper, J., et al. (2006). Hereditary parkinsonism with dementia is caused by mutations in ATP13A2, encoding a lysosomal type 5 P-type ATPase. Nat Genet 38, 1184-1191.

Ramonet, D., Podhajska, A., Stafa, K., Sonnay, S., Trancikova, A., Tsika, E., Pletnikova, O., Troncoso, J.C., Glauser, L., and Moore, D.J. (2012). PARK9-associated ATP13A2 localizes to intracellular acidic vesicles and regulates cation homeostasis and neuronal integrity. Hum Mol Genet.

Rockenstein, E., Schwach, G., Ingolic, E., Adame, A., Crews, L., Mante, M., Pfragner, R., Schreiner, E., Windisch, M., and Masliah, E. (2005). Lysosomal pathology associated with alpha-synuclein accumulation in transgenic models using an eGFP fusion protein. J Neurosci Res 80, 247-259.

Ross, C.A., and Poirier, M.A. (2005). Opinion: What is the role of protein aggregation in neurodegeneration? Nat Rev Mol Cell Biol 6, 891-898.

Sakaguchi-Nakashima, A., Meir, J.Y., Jin, Y., Matsumoto, K., and Hisamoto, N. (2007). LRK-1, a C. elegans PARK8-related kinase, regulates axonal-dendritic polarity of SV proteins. Curr Biol 17, 592598.

Samann, J., Hegermann, J., von Gromoff, E., Eimer, S., Baumeister, R., and Schmidt, E. (2009). Caenorhabditits elegans LRK-1 and PINK-1 act antagonistically in stress response and neurite outgrowth. J Biol Chem 284, 16482-16491.

Santel, A., and Frank, S. (2008). Shaping mitochondria: The complex posttranslational regulation of the mitochondrial fission protein DRP1. IUBMB Life 60, 448-455. 
Sauvanet, C., Duvezin-Caubet, S., di Rago, J.P., and Rojo, M. (2010). Energetic requirements and bioenergetic modulation of mitochondrial morphology and dynamics. Semin Cell Dev Biol 21, 558-565.

Sawin, E.R., Ranganathan, R., and Horvitz, H.R. (2000). C. elegans locomotory rate is modulated by the environment through a dopaminergic pathway and by experience through a serotonergic pathway. Neuron 26, 619-631.

Schafer, W.R. (2005). Egg-laying. WormBook, 1-7.

Schapira, A.H., Cooper, J.M., Dexter, D., Jenner, P., Clark, J.B., and Marsden, C.D. (1989). Mitochondrial complex I deficiency in Parkinson's disease. Lancet 1, 1269.

Scherz-Shouval, R., and Elazar, Z. (2011). Regulation of autophagy by ROS: physiology and pathology. Trends Biochem Sci 36, 30-38.

Schmidt, K., Wolfe, D.M., Stiller, B., and Pearce, D.A. (2009). Cd2+, Mn2+, Ni2+ and Se2+ toxicity to Saccharomyces cerevisiae lacking YPK9p the orthologue of human ATP13A2. Biochem Biophys Res Commun 383, 198-202.

Schroder, B., Wrocklage, C., Pan, C., Jager, R., Kosters, B., Schafer, H., Elsasser, H.P., Mann, M., and Hasilik, A. (2007). Integral and associated lysosomal membrane proteins. Traffic 8, 1676-1686.

Schultheis, P.J., Hagen, T.T., O'Toole, K.K., Tachibana, A., Burke, C.R., McGill, D.L., Okunade, G.W., and Shull, G.E. (2004). Characterization of the P5 subfamily of P-type transport ATPases in mice. Biochem Biophys Res Commun 323, 731-738.

Schulz, T.J., Zarse, K., Voigt, A., Urban, N., Birringer, M., and Ristow, M. (2007). Glucose restriction extends Caenorhabditis elegans life span by inducing mitochondrial respiration and increasing oxidative stress. Cell Metab 6, 280-293.

Schumacher, B., Hanazawa, M., Lee, M.H., Nayak, S., Volkmann, K., Hofmann, E.R., Hengartner, M., Schedl, T., and Gartner, A. (2005). Translational repression of C. elegans p53 by GLD-1 regulates DNA damage-induced apoptosis. Cell 120, 357-368.

Schwartz, H.T. (2007). A protocol describing pharynx counts and a review of other assays of apoptotic cell death in the nematode worm Caenorhabditis elegans. Nat Protoc 2, 705-714.

Senoo-Matsuda, N., Hartman, P.S., Akatsuka, A., Yoshimura, S., and Ishii, N. (2003). A complex II defect affects mitochondrial structure, leading to ced-3- and ced-4-dependent apoptosis and aging. J Biol Chem 278, 22031-22036.

Senoo-Matsuda, N., Yasuda, K., Tsuda, M., Ohkubo, T., Yoshimura, S., Nakazawa, H., Hartman, P.S., and Ishii, N. (2001). A defect in the cytochrome b large subunit in complex II causes both superoxide anion overproduction and abnormal energy metabolism in Caenorhabditis elegans. J Biol Chem 276, 41553-41558.

Serpell, L.C., Berriman, J., Jakes, R., Goedert, M., and Crowther, R.A. (2000). Fiber diffraction of synthetic alpha-synuclein filaments shows amyloid-like cross-beta conformation. Proc Natl Acad Sci U S A 97, 4897-4902.

Settembre, C., Fraldi, A., Jahreiss, L., Spampanato, C., Venturi, C., Medina, D., de Pablo, R., Tacchetti, C., Rubinsztein, D.C., and Ballabio, A. (2008). A block of autophagy in lysosomal storage disorders. Hum Mol Genet 17, 119-129.

Seydoux, G., and Greenwald, I. (1989). Cell autonomy of lin-12 function in a cell fate decision in C. elegans. Cell 57, 1237-1245.

Shamoto-Nagai, M., Maruyama, W., Akao, Y., Osawa, T., Tribl, F., Gerlach, M., Zucca, F.A., Zecca, L., Riederer, P., and Naoi, M. (2004). Neuromelanin inhibits enzymatic activity of $26 \mathrm{~S}$ proteasome in human dopaminergic SH-SY5Y cells. J Neural Transm 111, 1253-1265. 
Sharon, R., Bar-Joseph, I., Frosch, M.P., Walsh, D.M., Hamilton, J.A., and Selkoe, D.J. (2003). The formation of highly soluble oligomers of alpha-synuclein is regulated by fatty acids and enhanced in Parkinson's disease. Neuron 37, 583-595.

Sharon, R., Goldberg, M.S., Bar-Josef, I., Betensky, R.A., Shen, J., and Selkoe, D.J. (2001). alphaSynuclein occurs in lipid-rich high molecular weight complexes, binds fatty acids, and shows homology to the fatty acid-binding proteins. Proc Natl Acad Sci U S A 98, 9110-9115.

Shaw, R.J., Kosmatka, M., Bardeesy, N., Hurley, R.L., Witters, L.A., DePinho, R.A., and Cantley, L.C. (2004). The tumor suppressor LKB1 kinase directly activates AMP-activated kinase and regulates apoptosis in response to energy stress. Proc Natl Acad Sci U S A 101, 3329-3335.

Shendelman, S., Jonason, A., Martinat, C., Leete, T., and Abeliovich, A. (2004). DJ-1 is a redoxdependent molecular chaperone that inhibits alpha-synuclein aggregate formation. PLoS Biol 2, e362.

Sherer, T.B., Betarbet, R., and Greenamyre, J.T. (2002). Environment, mitochondria, and Parkinson's disease. Neuroscientist 8, 192-197.

Shimura, H., Hattori, N., Kubo, S., Mizuno, Y., Asakawa, S., Minoshima, S., Shimizu, N., Iwai, K., Chiba, T., Tanaka, K., et al. (2000). Familial Parkinson disease gene product, parkin, is a ubiquitinprotein ligase. Nat Genet 25, 302-305.

Shults, C.W. (2006). Lewy bodies. Proc Natl Acad Sci U S A 103, 1661-1668.

Silvestri, L., Caputo, V., Bellacchio, E., Atorino, L., Dallapiccola, B., Valente, E.M., and Casari, G. (2005). Mitochondrial import and enzymatic activity of PINK1 mutants associated to recessive parkinsonism. Hum Mol Genet 14, 3477-3492.

Sim, C.H., Lio, D.S., Mok, S.S., Masters, C.L., Hill, A.F., Culvenor, J.G., and Cheng, H.C. (2006). Cterminal truncation and Parkinson's disease-associated mutations down-regulate the protein serine/threonine kinase activity of PTEN-induced kinase-1. Hum Mol Genet 15, 3251-3262.

Singleton, A.B., Farrer, M., Johnson, J., Singleton, A., Hague, S., Kachergus, J., Hulihan, M., Peuralinna, T., Dutra, A., Nussbaum, R., et al. (2003). alpha-Synuclein locus triplication causes Parkinson's disease. Science 302, 841.

Smirnova, E., Griparic, L., Shurland, D.L., and van der Bliek, A.M. (2001). Dynamin-related protein Drp1 is required for mitochondrial division in mammalian cells. Mol Biol Cell 12, 2245-2256.

Smirnova, E., Shurland, D.L., Ryazantsev, S.N., and van der Bliek, A.M. (1998). A human dynaminrelated protein controls the distribution of mitochondria. J Cell Biol 143, 351-358.

Smith, W.W., Jiang, H., Pei, Z., Tanaka, Y., Morita, H., Sawa, A., Dawson, V.L., Dawson, T.M., and Ross, C.A. (2005). Endoplasmic reticulum stress and mitochondrial cell death pathways mediate A53T mutant alpha-synuclein-induced toxicity. Hum Mol Genet 14, 3801-3811.

Smith, W.W., Pei, Z., Jiang, H., Dawson, V.L., Dawson, T.M., and Ross, C.A. (2006). Kinase activity of mutant LRRK2 mediates neuronal toxicity. Nat Neurosci 9, 1231-1233.

Snyder, H., Mensah, K., Theisler, C., Lee, J., Matouschek, A., and Wolozin, B. (2003). Aggregated and monomeric alpha-synuclein bind to the $\mathrm{S} 6$ ' proteasomal protein and inhibit proteasomal function. $\mathrm{J}$ Biol Chem 278, 11753-11759.

Sohal, R.S., and Orr, W.C. (2012). The redox stress hypothesis of aging. Free Radic Biol Med 52, 539-555.

Souza, J.M., Giasson, B.I., Lee, V.M., and Ischiropoulos, H. (2000). Chaperone-like activity of synucleins. FEBS Lett $474,116-119$. 
Spillantini, M.G., Crowther, R.A., Jakes, R., Hasegawa, M., and Goedert, M. (1998). alpha-Synuclein in filamentous inclusions of Lewy bodies from Parkinson's disease and dementia with lewy bodies. Proc Natl Acad Sci U S A 95, 6469-6473.

Spillantini, M.G., Schmidt, M.L., Lee, V.M., Trojanowski, J.Q., Jakes, R., and Goedert, M. (1997). Alpha-synuclein in Lewy bodies. Nature 388, 839-840.

Springer, W., Hoppe, T., Schmidt, E., and Baumeister, R. (2005). A Caenorhabditis elegans Parkin mutant with altered solubility couples alpha-synuclein aggregation to proteotoxic stress. Hum Mol Genet 14, 3407-3423.

Springer, W., and Kahle, P.J. (2011). Regulation of PINK1-Parkin-mediated mitophagy. Autophagy 7, 266-278.

St-Pierre, J., Buckingham, J.A., Roebuck, S.J., and Brand, M.D. (2002). Topology of superoxide production from different sites in the mitochondrial electron transport chain. J Biol Chem 277, 4478444790.

Stefanis, L. (2012). alpha-Synuclein in Parkinson's Disease. Cold Spring Harb Perspect Med 2, a009399.

Stefanis, L., Larsen, K.E., Rideout, H.J., Sulzer, D., and Greene, L.A. (2001). Expression of A53T mutant but not wild-type alpha-synuclein in PC12 cells induces alterations of the ubiquitin-dependent degradation system, loss of dopamine release, and autophagic cell death. J Neurosci 21, 9549-9560.

Sternberg, P.W., and Horvitz, H.R. (1986). Pattern formation during vulval development in C. elegans. Cell 44, 761-772.

Sulston, J., Dew, M., and Brenner, S. (1975). Dopaminergic neurons in the nematode Caenorhabditis elegans. J Comp Neurol 163, 215-226.

Sulston, J.E., Schierenberg, E., White, J.G., and Thomson, J.N. (1983). The embryonic cell lineage of the nematode Caenorhabditis elegans. Dev Biol 100, 64-119.

Sumakovic, M., Hegermann, J., Luo, L., Husson, S.J., Schwarze, K., Olendrowitz, C., Schoofs, L., Richmond, J., and Eimer, S. (2009). UNC-108/RAB-2 and its effector RIC-19 are involved in dense core vesicle maturation in Caenorhabditis elegans. J Cell Biol 186, 897-914.

Takeda, A., Mallory, M., Sundsmo, M., Honer, W., Hansen, L., and Masliah, E. (1998). Abnormal accumulation of NACP/alpha-synuclein in neurodegenerative disorders. Am J Pathol 152, 367-372.

Tan, J., Zhang, T., Jiang, L., Chi, J., Hu, D., Pan, Q., Wang, D., and Zhang, Z. (2011). Regulation of intracellular manganese homeostasis by Kufor-Rakeb syndrome-associated ATP13A2 protein. J Biol Chem 286, 29654-29662.

Tanaka, A. (2010). Parkin-mediated selective mitochondrial autophagy, mitophagy: Parkin purges damaged organelles from the vital mitochondrial network. FEBS Lett 584, 1386-1392.

Tipton, K.F., and Singer, T.P. (1993). Advances in our understanding of the mechanisms of the neurotoxicity of MPTP and related compounds. J Neurochem 61, 1191-1206.

Toulouse, A., and Sullivan, A.M. (2008). Progress in Parkinson's disease-where do we stand? Prog Neurobiol 85, 376-392.

Twig, G., Elorza, A., Molina, A.J., Mohamed, H., Wikstrom, J.D., Walzer, G., Stiles, L., Haigh, S.E., Katz, S., Las, G., et al. (2008a). Fission and selective fusion govern mitochondrial segregation and elimination by autophagy. EMBO J 27, 433-446.

Twig, G., Hyde, B., and Shirihai, O.S. (2008b). Mitochondrial fusion, fission and autophagy as a quality control axis: the bioenergetic view. Biochim Biophys Acta 1777, 1092-1097. 
Ugolino, J., Fang, S., Kubisch, C., and Monteiro, M.J. (2011). Mutant Atp13a2 proteins involved in parkinsonism are degraded by ER-associated degradation and sensitize cells to ER-stress induced cell death. Hum Mol Genet 20, 3565-3577.

Uversky, V.N., Li, J., and Fink, A.L. (2001). Metal-triggered structural transformations, aggregation, and fibrillation of human alpha-synuclein. A possible molecular NK between Parkinson's disease and heavy metal exposure. J Biol Chem 276, 44284-44296.

Uversky, V.N., Li, J., Souillac, P., Millett, I.S., Doniach, S., Jakes, R., Goedert, M., and Fink, A.L. (2002). Biophysical properties of the synucleins and their propensities to fibrillate: inhibition of alphasynuclein assembly by beta- and gamma-synucleins. J Biol Chem 277, 11970-11978.

Valente, E.M., Abou-Sleiman, P.M., Caputo, V., Muqit, M.M., Harvey, K., Gispert, S., Ali, Z., Del Turco, D., Bentivoglio, A.R., Healy, D.G., et al. (2004a). Hereditary early-onset Parkinson's disease caused by mutations in PINK1. Science 304, 1158-1160.

Valente, E.M., Salvi, S., lalongo, T., Marongiu, R., Elia, A.E., Caputo, V., Romito, L., Albanese, A., Dallapiccola, B., and Bentivoglio, A.R. (2004b). PINK1 mutations are associated with sporadic earlyonset parkinsonism. Ann Neurol 56, 336-341.

van Ham, T.J., Esposito, A., Kumita, J.R., Hsu, S.T., Kaminski Schierle, G.S., Kaminski, C.F., Dobson, C.M., Nollen, E.A., and Bertoncini, C.W. (2010). Towards multiparametric fluorescent imaging of amyloid formation: studies of a YFP model of alpha-synuclein aggregation. J Mol Biol 395, 627-642.

van Ham, T.J., Thijssen, K.L., Breitling, R., Hofstra, R.M., Plasterk, R.H., and Nollen, E.A. (2008). C. elegans model identifies genetic modifiers of alpha-synuclein inclusion formation during aging. PLoS Genet 4, e1000027.

Van Voorhies, W.A. (2002). The influence of metabolic rate on longevity in the nematode Caenorhabditis elegans. Aging Cell 1, 91-101.

Varkey, J., Isas, J.M., Mizuno, N., Jensen, M.B., Bhatia, V.K., Jao, C.C., Petrlova, J., Voss, J.C., Stamou, D.G., Steven, A.C., et al. (2010). Membrane curvature induction and tubulation are common features of synucleins and apolipoproteins. J Biol Chem 285, 32486-32493.

Ved, R., Saha, S., Westlund, B., Perier, C., Burnam, L., Sluder, A., Hoener, M., Rodrigues, C.M., Alfonso, A., Steer, C., et al. (2005). Similar patterns of mitochondrial vulnerability and rescue induced by genetic modification of alpha-synuclein, parkin, and DJ-1 in Caenorhabditis elegans. J Biol Chem $280,42655-42668$

Vergarajauregui, S., Connelly, P.S., Daniels, M.P., and Puertollano, R. (2008). Autophagic dysfunction in mucolipidosis type IV patients. Hum Mol Genet 17, 2723-2737.

Vila, M., and Przedborski, S. (2004). Genetic clues to the pathogenesis of Parkinson's disease. Nat Med 10 Suppl, S58-62.

Vives-Bauza, C., and Przedborski, S. (2011). Mitophagy: the latest problem for Parkinson's disease. Trends Mol Med 17, 158-165.

Vives-Bauza, C., Tocilescu, M., Devries, R.L., Alessi, D.M., Jackson-Lewis, V., and Przedborski, S. (2010a). Control of mitochondrial integrity in Parkinson's disease. Prog Brain Res 183, 99-113.

Vives-Bauza, C., Zhou, C., Huang, Y., Cui, M., de Vries, R.L., Kim, J., May, J., Tocilescu, M.A., Liu, W., Ko, H.S., et al. (2010b). PINK1-dependent recruitment of Parkin to mitochondria in mitophagy. Proc Natl Acad Sci U S A 107, 378-383.

Volles, M.J., and Lansbury, P.T., Jr. (2003). Zeroing in on the pathogenic form of alpha-synuclein and its mechanism of neurotoxicity in Parkinson's disease. Biochemistry 42, 7871-7878. 
Volles, M.J., Lee, S.J., Rochet, J.C., Shtilerman, M.D., Ding, T.T., Kessler, J.C., and Lansbury, P.T., Jr. (2001). Vesicle permeabilization by protofibrillar alpha-synuclein: implications for the pathogenesis and treatment of Parkinson's disease. Biochemistry 40, 7812-7819.

Wang, K., and Klionsky, D.J. (2011). Mitochondria removal by autophagy. Autophagy 7, 297-300.

Waterham, H.R., Koster, J., van Roermund, C.W., Mooyer, P.A., Wanders, R.J., and Leonard, J.V. (2007). A lethal defect of mitochondrial and peroxisomal fission. N Engl J Med 356, 1736-1741.

Webb, J.L., Ravikumar, B., Atkins, J., Skepper, J.N., and Rubinsztein, D.C. (2003). Alpha-Synuclein is degraded by both autophagy and the proteasome. J Biol Chem 278, 25009-25013.

Weinreb, P.H., Zhen, W., Poon, A.W., Conway, K.A., and Lansbury, P.T., Jr. (1996). NACP, a protein implicated in Alzheimer's disease and learning, is natively unfolded. Biochemistry 35, 13709-13715.

West, A.B., Moore, D.J., Biskup, S., Bugayenko, A., Smith, W.W., Ross, C.A., Dawson, V.L., and Dawson, T.M. (2005). Parkinson's disease-associated mutations in leucine-rich repeat kinase 2 augment kinase activity. Proc Natl Acad Sci U S A 102, 16842-16847.

West, A.B., Moore, D.J., Choi, C., Andrabi, S.A., Li, X., Dikeman, D., Biskup, S., Zhang, Z., Lim, K.L., Dawson, V.L., et al. (2007). Parkinson's disease-associated mutations in LRRK2 link enhanced GTPbinding and kinase activities to neuronal toxicity. Hum Mol Genet 16, 223-232.

Westermann, B. (2010a). Mitochondrial dynamics in model organisms: what yeasts, worms and flies have taught us about fusion and fission of mitochondria. Semin Cell Dev Biol 21, 542-549.

Westermann, B. (2010b). Mitochondrial fusion and fission in cell life and death. Nat Rev Mol Cell Biol $11,872-884$.

Williams, D.R., Hadeed, A., al-Din, A.S., Wreikat, A.L., and Lees, A.J. (2005). Kufor Rakeb disease: autosomal recessive, levodopa-responsive parkinsonism with pyramidal degeneration, supranuclear gaze palsy, and dementia. Mov Disord 20, 1264-1271.

Winner, B., Jappelli, R., Maji, S.K., Desplats, P.A., Boyer, L., Aigner, S., Hetzer, C., Loher, T., Vilar, M., Campioni, S., et al. (2011). In vivo demonstration that alpha-synuclein oligomers are toxic. Proc Natl Acad Sci U S A 108, 4194-4199.

Winslow, A.R., Chen, C.W., Corrochano, S., Acevedo-Arozena, A., Gordon, D.E., Peden, A.A., Lichtenberg, M., Menzies, F.M., Ravikumar, B., Imarisio, S., et al. (2010). alpha-Synuclein impairs macroautophagy: implications for Parkinson's disease. J Cell Biol 190, 1023-1037.

Witte, K., Schuh, A.L., Hegermann, J., Sarkeshik, A., Mayers, J.R., Schwarze, K., Yates, J.R., 3rd, Eimer, S., and Audhya, A. (2011). TFG-1 function in protein secretion and oncogenesis. Nat Cell Biol $13,550-558$.

Xilouri, M., Vogiatzi, T., Vekrellis, K., Park, D., and Stefanis, L. (2009). Abberant alpha-synuclein confers toxicity to neurons in part through inhibition of chaperone-mediated autophagy. PLoS One 4, e5515.

Yang, Y., Gehrke, S., Imai, Y., Huang, Z., Ouyang, Y., Wang, J.W., Yang, L., Beal, M.F., Vogel, H., and Lu, B. (2006). Mitochondrial pathology and muscle and dopaminergic neuron degeneration caused by inactivation of Drosophila Pink1 is rescued by Parkin. Proc Natl Acad Sci U S A 103, 10793-10798.

Yang, Y., Nishimura, I., Imai, Y., Takahashi, R., and Lu, B. (2003). Parkin suppresses dopaminergic neuron-selective neurotoxicity induced by Pael-R in Drosophila. Neuron 37, 911-924.

Yokota, T., Sugawara, K., Ito, K., Takahashi, R., Ariga, H., and Mizusawa, H. (2003). Down regulation of DJ-1 enhances cell death by oxidative stress, ER stress, and proteasome inhibition. Biochem Biophys Res Commun 312, 1342-1348. 
Yoneda, T., Benedetti, C., Urano, F., Clark, S.G., Harding, H.P., and Ron, D. (2004). Compartmentspecific perturbation of protein handling activates genes encoding mitochondrial chaperones. J Cell Sci 117, 4055-4066.

Yoshino, H., Nakagawa-Hattori, Y., Kondo, T., and Mizuno, Y. (1992). Mitochondrial complex I and II activities of lymphocytes and platelets in Parkinson's disease. J Neural Transm Park Dis Dement Sect 4, 27-34.

Youdim, M.B., Ben-Shachar, D., and Riederer, P. (1994). The enigma of neuromelanin in Parkinson's disease substantia nigra. J Neural Transm Suppl 43, 113-122.

Youle, R.J., and Karbowski, M. (2005). Mitochondrial fission in apoptosis. Nat Rev Mol Cell Biol 6, 657-663.

Zarranz, J.J., Alegre, J., Gomez-Esteban, J.C., Lezcano, E., Ros, R., Ampuero, I., Vidal, L., Hoenicka, J., Rodriguez, O., Atares, B., et al. (2004). The new mutation, E46K, of alpha-synuclein causes Parkinson and Lewy body dementia. Ann Neurol 55, 164-173.

Zhang, Y., Gao, J., Chung, K.K., Huang, H., Dawson, V.L., and Dawson, T.M. (2000). Parkin functions as an E2-dependent ubiquitin- protein ligase and promotes the degradation of the synaptic vesicleassociated protein, CDCrel-1. Proc Natl Acad Sci U S A 97, 13354-13359.

Zhang, Y., Qi, H., Taylor, R., Xu, W., Liu, L.F., and Jin, S. (2007). The role of autophagy in mitochondria maintenance: characterization of mitochondrial functions in autophagy-deficient $S$. cerevisiae strains. Autophagy 3, 337-346.

Zhao, Q., Wang, J., Levichkin, I.V., Stasinopoulos, S., Ryan, M.T., and Hoogenraad, N.J. (2002). A mitochondrial specific stress response in mammalian cells. EMBO J 21, 4411-4419.

Zhou, Z., Hartwieg, E., and Horvitz, H.R. (2001). CED-1 is a transmembrane receptor that mediates cell corpse engulfment in C. elegans. Cell 104, 43-56.

Zhu, P.P., Patterson, A., Stadler, J., Seeburg, D.P., Sheng, M., and Blackstone, C. (2004). Intra- and intermolecular domain interactions of the C-terminal GTPase effector domain of the multimeric dynamin-like GTPase Drp1. J Biol Chem 279, 35967-35974.

Zimprich, A., Muller-Myhsok, B., Farrer, M., Leitner, P., Sharma, M., Hulihan, M., Lockhart, P., Strongosky, A., Kachergus, J., Calne, D.B., et al. (2004). The PARK8 locus in autosomal dominant parkinsonism: confirmation of linkage and further delineation of the disease-containing interval. Am J Hum Genet 74, 11-19.

Zoncu, R., Bar-Peled, L., Efeyan, A., Wang, S., Sancak, Y., and Sabatini, D.M. (2011). mTORC1 senses lysosomal amino acids through an inside-out mechanism that requires the vacuolar $\mathrm{H}(+)$ ATPase. Science 334, 678-683.

Zuchner, S., Mersiyanova, I.V., Muglia, M., Bissar-Tadmouri, N., Rochelle, J., Dadali, E.L., Zappia, M., Nelis, E., Patitucci, A., Senderek, J., et al. (2004). Mutations in the mitochondrial GTPase mitofusin 2 cause Charcot-Marie-Tooth neuropathy type 2A. Nat Genet 36, 449-451. 


\section{Appendix}

\subsection{Alignment of C. elegans CATP-6 and human ATP13A2}

Alignment of the protein sequences of $C$. elegans CATP-- 6 and human ATP13A2 was performed using the program MUSCLE and displayed using the program BOXSHADE (http://www.ch.embnet.org/software/BOX_form.html).

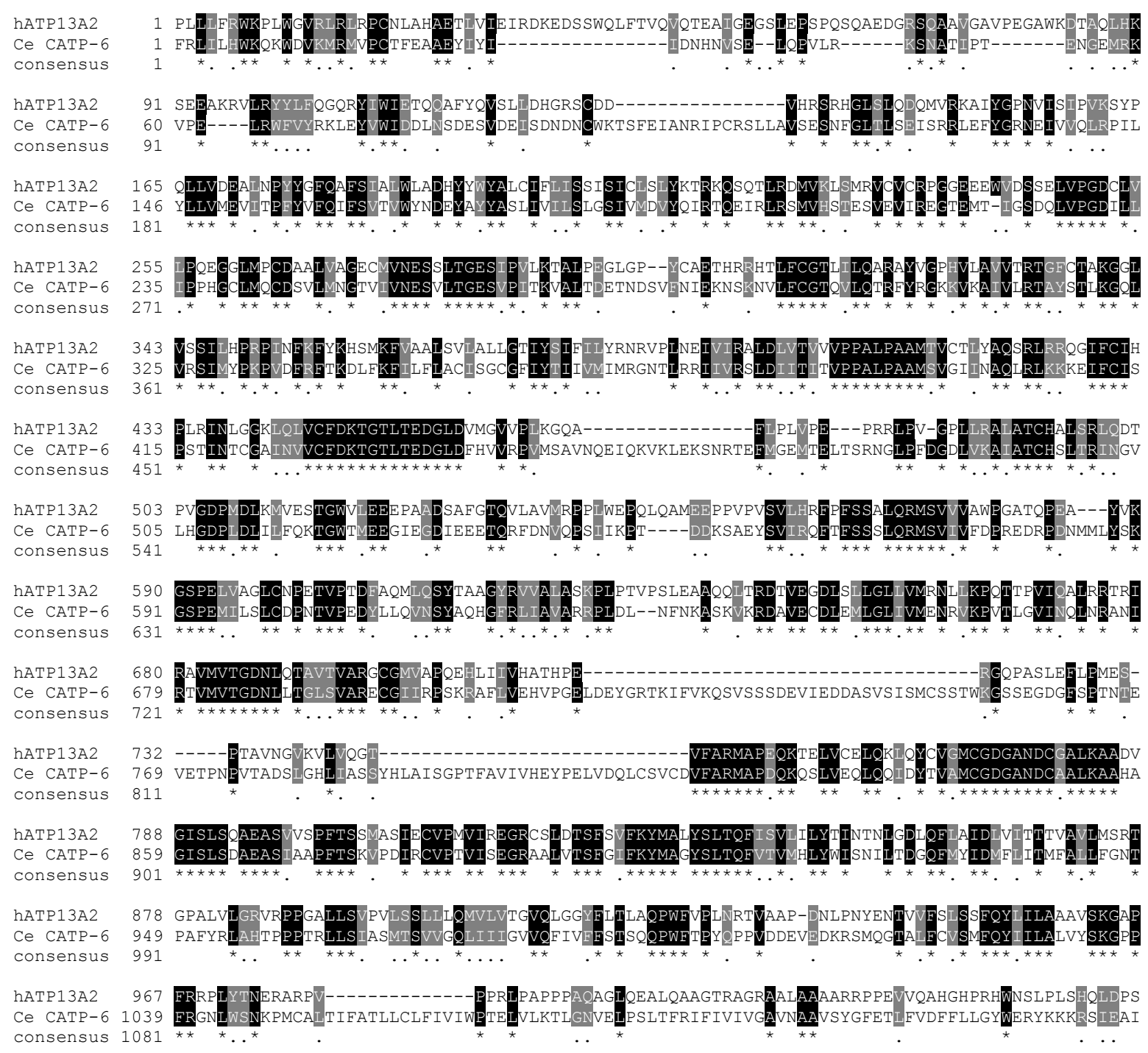

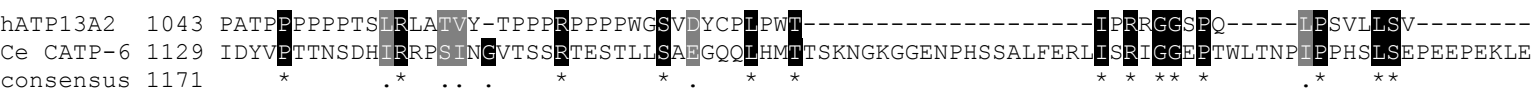




\subsection{Plasmids, primers and strains}

Table 8.1: List of plasmids used in this study.

\begin{tabular}{|c|c|c|c|}
\hline Plasmid name & Resistance & Description & Source \\
\hline pBluescript II SK(+) & $A m p^{R}$ & Entry vector for cloning & Stratagene \\
\hline pGEM-T & $A m p^{H}$ & Entry vector for TA cloning & Promega \\
\hline L4440 & $A m p^{R}$ & Entry vector for feeding RNAi & Addgene \\
\hline $\begin{array}{l}\text { pPD115.62 } \\
\text { (pmyo-3::gfp) }\end{array}$ & $A m p^{R}$ & $\begin{array}{l}\text { Muscle expression vector for } C \text {. elegans } \\
\text { contains unc-54 3' UTR }\end{array}$ & $\begin{array}{l}\text { Addgene, Andrew } \\
\text { Fire vector kit }\end{array}$ \\
\hline pmyo-3::yfp & $A m p^{R}$ & $\begin{array}{l}\text { pPD115.62 in which gfp was replaced by } \\
\text { yfp }\end{array}$ & $\begin{array}{l}\text { Kindly provided by } \\
\text { Dr. Ling Luo }\end{array}$ \\
\hline prab-3::mcherry & $A m p^{R}$ & $\begin{array}{l}\text { Neuronal expression vector for } C \text {. elegans } \\
\text { based on pPD155.62 backbone }\end{array}$ & $\begin{array}{l}\text { Kindly provided by } \\
\text { Dr. Marija Suma- } \\
\text { kovic }\end{array}$ \\
\hline pmyo-3::gfp-grip & $A m p^{R}$ & $\begin{array}{l}\text { Muscle expression vector for } C \text {. elegans } \\
\text { based on pPD115.62 backbone } \\
\text { for N-terminal Tags }\end{array}$ & $\begin{array}{l}\text { Kindly provided by } \\
\text { Dr. Ling Luo }\end{array}$ \\
\hline pT7_7 wt $\alpha S$ & $A m p^{R}$ & used as template for $\alpha S$ PCR & $\begin{array}{l}\text { Kindly provided by } \\
\text { Prof. Markus } \\
\text { Zweckstetter }\end{array}$ \\
\hline pT7_7 TP $\alpha S$ & $A m p^{R}$ & used as template for $\alpha S$ PCR & $\begin{array}{l}\text { Kindly provided by } \\
\text { Prof Markus } \\
\text { Zweckstetter }\end{array}$ \\
\hline pT7_7 A56P $\alpha S$ & $A m p^{R}$ & used as template for $\alpha S$ PCR & $\begin{array}{l}\text { Kindly provided by } \\
\text { Prof Markus } \\
\text { Zweckstetter }\end{array}$ \\
\hline pT7_7 A3OP $\alpha S$ & $A m p^{R}$ & used as template for $\alpha S$ PCR & $\begin{array}{l}\text { Kindly provided by } \\
\text { Prof Markus } \\
\text { Zweckstetter }\end{array}$ \\
\hline pT7_7 A53T aS & $A m p^{R}$ & used as template for $\alpha S$ PCR & $\begin{array}{l}\text { Kindly provided by } \\
\text { Prof Markus } \\
\text { Zweckstetter }\end{array}$ \\
\hline pQL41 & $A m p^{R}$ & TagRFP_T vector & Ephrogen \\
\hline pdat-1::mrfp & $A m p^{R}$ & $\begin{array}{l}\text { expression of mRFP in C. elegans DA } \\
\text { neurons }\end{array}$ & Dr. Stefan Eimer \\
\hline pdat-1::egfp & $A m p^{R}$ & $\begin{array}{l}\text { expression of eGFP in C. elegans DA } \\
\text { neurons }\end{array}$ & Dr. Stefan Eimer \\
\hline pdat-1::wt $\alpha S$ & $\mathrm{Amp}^{\mathrm{R}}$ & $\begin{array}{l}\text { expression of wt } \alpha \mathrm{S} \text { in } C \text {. elegans DA } \\
\text { neurons }\end{array}$ & Dr. Stefan Eimer \\
\hline pdat-1::A56P $\alpha S$ & $A m p^{R}$ & $\begin{array}{l}\text { expression of A56P aS in C. elegans DA } \\
\text { neurons }\end{array}$ & Dr. Stefan Eimer \\
\hline pdat-1::TP $\alpha S$ & $A m p^{R}$ & $\begin{array}{l}\text { expression of TP } \alpha \mathrm{S} \text { in C. elegans DA } \\
\text { neurons }\end{array}$ & Dr. Stefan Eimer \\
\hline pdat-1::A30P aS & $A m p^{R}$ & $\begin{array}{l}\text { expression of A30P aS in C. elegans DA } \\
\text { neurons }\end{array}$ & Dr. Stefan Eimer \\
\hline pdat-1::A53T aS & $A m p^{R}$ & $\begin{array}{l}\text { expression of A53T aS in C. elegans DA } \\
\text { neurons }\end{array}$ & Dr. Stefan Eimer \\
\hline pmyo-3::wt $\alpha S-m y f p$ & $A m p^{R}$ & $\begin{array}{l}\text { expression of wt } \alpha \mathrm{S}-\mathrm{YFP} \text { in } C \text {. elegans } \\
\text { muscle cells }\end{array}$ & $\begin{array}{l}\text { Cloned in this } \\
\text { study }\end{array}$ \\
\hline pmyo-3::TP $\alpha S-m y f p$ & $A m p^{R}$ & $\begin{array}{l}\text { expression of TP } \alpha \mathrm{S}-\mathrm{YFP} \text { in } C \text {. elegans } \\
\text { muscle cells }\end{array}$ & $\begin{array}{l}\text { Cloned in this } \\
\text { study }\end{array}$ \\
\hline $\begin{array}{l}\text { pmyo-3::A30P } \alpha S- \\
\text { myfp }\end{array}$ & $A m p^{R}$ & $\begin{array}{l}\text { expression of A30P aS-YFP in C. elegans } \\
\text { muscle cells }\end{array}$ & $\begin{array}{l}\text { Cloned in this } \\
\text { study }\end{array}$ \\
\hline $\begin{array}{l}\text { pmyo-3::A53T } \alpha S- \\
\text { myfp }\end{array}$ & $A m p^{R}$ & $\begin{array}{l}\text { expression of A53T aS-YFP in C. elegans } \\
\text { muscle cells }\end{array}$ & $\begin{array}{l}\text { Cloned in this } \\
\text { study }\end{array}$ \\
\hline pmyo-3::tom70-cfp & $A m p^{R}$ & $\begin{array}{l}\text { marker for outer mitochondrial membrane } \\
\text { (TM domain of yeast TOM70 fused to } \\
\text { CFP) in C. elegans muscle cells }\end{array}$ & $\begin{array}{l}\text { Kindly provided by } \\
\text { Alexander van der } \\
\text { Bliek }\end{array}$ \\
\hline
\end{tabular}




\begin{tabular}{|c|c|c|c|}
\hline prab-3::wt $\alpha S-m y f p$ & $A m p^{R}$ & $\begin{array}{l}\text { expression of wt } \alpha \mathrm{S}-\mathrm{YFP} \text { in } C \text {. elegans } \\
\text { neurons }\end{array}$ & $\begin{array}{l}\text { Cloned in this } \\
\text { study }\end{array}$ \\
\hline prab-3::tom70-cfp & $A m p^{R}$ & $\begin{array}{l}\text { marker for outer mitochondrial membrane } \\
\text { (TM domain of yeast TOM70 fused to } \\
\text { CFP) in C. elegans neurons }\end{array}$ & $\begin{array}{l}\text { Cloned in this } \\
\text { study }\end{array}$ \\
\hline pmyo-3::tagrfp-drp-1 & $A m p^{R}$ & $\begin{array}{l}\text { expression of TagRFP-DRP-1 in } \\
\text { C. elegans muscle cells }\end{array}$ & $\begin{array}{l}\text { Cloned in this } \\
\text { study }\end{array}$ \\
\hline prab-3::tagrfp-drp-1 & $A m p^{R}$ & $\begin{array}{l}\text { expression of TagRFP-DRP-1 in } \\
\text { C. elegans neurons }\end{array}$ & $\begin{array}{l}\text { Cloned in this } \\
\text { study }\end{array}$ \\
\hline pcatp-6::gfp & $A m p^{R}$ & For expression pattern analysis of catp- 6 & $\begin{array}{l}\text { Kindly provided by } \\
\text { Katrin Schwarze }\end{array}$ \\
\hline pmyo-3::catp-6 & $A m p^{R}$ & $\begin{array}{l}\text { Expression of CATP- } 6 \text { in C. elegans mus- } \\
\text { cle cells } \\
\text { pPD115.62 backbone }\end{array}$ & $\begin{array}{l}\text { Kindly provided by } \\
\text { Katrin Schwarze }\end{array}$ \\
\hline L4440 drp-1 & $A m p^{R}$ & For feeding RNAi against $d r p-1$ & $\begin{array}{l}\text { Cloned in this } \\
\text { study }\end{array}$ \\
\hline L4440 fis- 1 & $A m p^{R}$ & For feeding RNAi against fis-1 & $\begin{array}{l}\text { Cloned in this } \\
\text { study }\end{array}$ \\
\hline L4440 fzo-1 & $A m p^{R}$ & For feeding RNAi against $f z o-1$ & $\begin{array}{l}\text { Cloned in this } \\
\text { study }\end{array}$ \\
\hline L4440 eat-3 & $\mathrm{Amp}^{\mathrm{R}}$ & For feeding RNAi against eat-3 & $\begin{array}{l}\text { Cloned in this } \\
\text { study }\end{array}$ \\
\hline L4440 pos-1 & $\mathrm{Amp}^{\mathrm{K}}$ & For feeding RNAi against pos-1 & Kamath, 2003 \\
\hline $\begin{array}{l}\text { pGEM-T tagrfp_t w/o } \\
\text { stop }\end{array}$ & $A m p^{R}$ & $\begin{array}{l}\text { TagRFP_T in Entry vector for subsequent } \\
\text { cloning }\end{array}$ & $\begin{array}{l}\text { Cloned in this } \\
\text { study }\end{array}$ \\
\hline
\end{tabular}

Table 8.2: Cloning strategies for constructs used in this study.

\begin{tabular}{|c|c|c|}
\hline Plasmid name & PCR & Cloning \\
\hline pGEM-T wt $\alpha S$ & $\begin{array}{l}\text { template: pT7_7 wt } \alpha S \\
\text { primers: oGQ1340/oGQ1341 } \\
\text { polymerase: Taq }\end{array}$ & $\begin{array}{l}\text { Ligate PCR product into pGEM-T } \\
\text { (AT cloning) }\end{array}$ \\
\hline pGEM-T TP $\alpha S$ & $\begin{array}{l}\text { template: } \mathrm{pT7} 7 \mathrm{7P} \alpha \mathrm{SS} \\
\text { primers: oGQ1340/oGQ1341 } \\
\text { polymerase: Taq }\end{array}$ & $\begin{array}{l}\text { Ligate PCR product into pGEM-T } \\
\text { (AT cloning) }\end{array}$ \\
\hline pGEM-T $A 30 P \alpha S$ & $\begin{array}{l}\text { template: } \mathrm{pT7} 7 \mathrm{A30P} \alpha \mathrm{S} \\
\text { primers: oGQ1340/oGQ1341 } \\
\text { Polymerase: Taq }\end{array}$ & $\begin{array}{l}\text { Ligate PCR product into pGEM-T } \\
\text { (AT cloning) }\end{array}$ \\
\hline pGEM-T A53T $\alpha S$ & $\begin{array}{l}\text { template: pT7_7 A53T } \alpha S \\
\text { primers: oGQ1340/oGQ1341 } \\
\text { polymerase: Taq }\end{array}$ & $\begin{array}{l}\text { Ligate PCR product into pGEM-T } \\
\text { (AT cloning) }\end{array}$ \\
\hline Pmyo-3::wt $\alpha$ S-myfp & & $\begin{array}{l}\text { wt } \alpha S \text { from pGEM-T wt } \alpha S \text { cloned } \\
\text { into pmyo-3::myfp (Kpnl/Notl) }\end{array}$ \\
\hline pmyo-3::TP $\alpha S-m y f p$ & & $\begin{array}{l}\text { wt } \alpha S \text { in pmyo-3::wt } \alpha S-m y f p \text { re- } \\
\text { placed by TP } \alpha S \text { from pGEM-T TP } \\
\alpha S \text { (Kpnl/Notl) }\end{array}$ \\
\hline pmyo-3::A30P aS-myfp & & $\begin{array}{l}\text { wt } \alpha S \text { in pmyo-3::wt } \alpha S-m y f p \text { re- } \\
\text { placed by } A 30 P \alpha S \text { from pGEM-T } \\
\text { A3OP } \alpha S \text { (Kpnl/Notl) }\end{array}$ \\
\hline pmyo-3::A53T aS-myfp & & $\begin{array}{l}\text { wt } \alpha S \text { in pmyo-3::wt } \alpha S-m y f p \text { re- } \\
\text { placed by } A 53 T \alpha S \text { from pGEM-T } \\
\text { A53T } \alpha S \text { (Kpnl/Notl) }\end{array}$ \\
\hline prab-3::wt $\alpha S-m y f p$ & & $\begin{array}{l}\text { pmyo-3 in pmyo-3::wt } \alpha S-m y f p \\
\text { replaced by prab-3 from } \\
\text { prab-3::mcherry (Pstl/Kpnl) }\end{array}$ \\
\hline prab-3::tom70-cfp & & $\begin{array}{l}\text { pmyo-3 in pmyo-3::tom70-cfp re- } \\
\text { placed by prab-3 from } \\
\text { prab-3::mcherry (Pstl/Kpnl) }\end{array}$ \\
\hline
\end{tabular}




\begin{tabular}{|c|c|c|}
\hline pGEM-T tagrfp (w/o stop) & $\begin{array}{l}\text { template: pQL41 } \\
\text { primers: oGQ456/539 } \\
\text { polymerase: Taq }\end{array}$ & $\begin{array}{l}\text { TagRFP_T cloned into pGEM-T } \\
\text { (AT cloning) }\end{array}$ \\
\hline pBluescript II SK(+) $d r p-1$ & $\begin{array}{l}\text { template: C. elegans genomic DNA } \\
\text { primers: oGQ1216/oGQ1217 } \\
\text { polymerase: Pfu }\end{array}$ & $\begin{array}{l}\text { PCR product cloned into } \\
\text { pBluescript II SK(+) (Xhol/BamHI) }\end{array}$ \\
\hline pmyo-3::tagrfp-drp-1 & & $\begin{array}{l}\text { tagrfp from pGEM-T tagrfp (w/o } \\
\text { stop) (Kpnl/Notl) and drp-1 from } \\
\text { pBluescript II SK(+) drp-1 } \\
\text { (Notl/Xhol) cloned into } \\
\text { pmyo-3::gfp-grip (Kpnl/Xhol) }\end{array}$ \\
\hline prab-3::tagrfp-drp-1 & & $\begin{array}{l}\text { tagrfp from pGEM-T tagrfp (w/o } \\
\text { stop) (Kpnl/Notl) and drp-1 from } \\
\text { pBluescript II SK(+) drp-1 } \\
\text { (Notl/Xhol) cloned into } \\
\text { prab-3::mcherry (Kpnl/Xhol) }\end{array}$ \\
\hline L4440 drp-1 & & $\begin{array}{l}\text { drp-1 from pBluescript II SK(+) } \\
\text { cloned into L4440 (Notl/Xhol) }\end{array}$ \\
\hline L4440 fis-1 & $\begin{array}{l}\text { template: C. elegans RB3 cDNA } \\
\text { library } \\
\text { primers: oGQ1725/oGQ1726 } \\
\text { polymerase: Taq }\end{array}$ & $\begin{array}{l}\text { PCR product ligated in L4444 cut } \\
\text { with EcoRV }\end{array}$ \\
\hline L4440 fzo-1 & $\begin{array}{l}\text { template: C. elegans RB1 cDNA } \\
\text { library } \\
\text { primers: oGQ1727/1728 } \\
\text { polymerase: Pfu }\end{array}$ & $\begin{array}{l}\text { PCR product ligated in L4444 cut } \\
\text { with EcoRV }\end{array}$ \\
\hline L4440 eat-3 & $\begin{array}{l}\text { template: C. elegans RB3 cDNA } \\
\text { library } \\
\text { primers: oGQ1723/1724 } \\
\text { polymerase: Pfu }\end{array}$ & $\begin{array}{l}\text { PCR product cloned into L4440 } \\
\text { (Notl/Xhol) }\end{array}$ \\
\hline
\end{tabular}

Table 8.3: List of primers used in this study.

\begin{tabular}{|c|c|c|c|}
\hline Primer & Description & Sequence & $T_{m}$ \\
\hline oGQ456 & $\begin{array}{l}\text { TagRFP forward primer that matches the } \\
\text { Start codon and introduces a BamHI and } \\
\text { Kpnl site upstream }\end{array}$ & $\begin{array}{l}\text { ggatccggtaccgaaaaaatgag- } \\
\text { tgtgtctaagggcgaagagctg }\end{array}$ & $72^{\circ} \mathrm{C}$ \\
\hline oGQ539 & $\begin{array}{l}\text { TagRFP reverse primer that matches the end } \\
\text { of TagRFP and introduces a Notl, Nhel, } \\
\text { Eco } 47 \text { III and Agel site afterwards }\end{array}$ & $\begin{array}{l}\text { ccctaccggtgaa- } \\
\text { gcgctagcgcggccgcctccg- } \\
\text { gaattaagtttgtgcc }\end{array}$ & $80^{\circ} \mathrm{C}$ \\
\hline oGQ1216 & $\begin{array}{l}\text { fwd primer for drp-1 genomic DNA that intro- } \\
\text { duces a Eco47III half site and BamHI site } \\
\text { upstream of start ATG }\end{array}$ & $\begin{array}{l}\text { gctg- } \\
\text { gatcCATGGAAAATCTCATTCCT } \\
\text { GTCGTC }\end{array}$ & $57^{\circ} \mathrm{C}$ \\
\hline oGQ1217 & $\begin{array}{l}\text { rev primer drp-1 genomic DNA that introduc- } \\
\text { es Smal and Xhol sites after stop codon }\end{array}$ & $\begin{array}{l}\text { ccccccgggctcgagCAA- } \\
\text { GCATCATCAAAGAATTTCAT- } \\
\text { ATAAATCC }\end{array}$ & $56^{\circ} \mathrm{C}$ \\
\hline oGQ1340 & $\begin{array}{l}\text { for Primer for human } \alpha \text {-synuclein (w/o stop) } \\
\text { that introduces Kpn I and Age I sites up- } \\
\text { stream of the start codon (BamHI in the } \\
\text { gene) }\end{array}$ & $\begin{array}{l}\text { gggggtaccgg- } \\
\text { taATGGATGTATTCATGAAAG } \\
\text { GACTTTC }\end{array}$ & $55^{\circ} \mathrm{C}$ \\
\hline oGQ1341 & $\begin{array}{l}\text { rev Primer for human } \alpha \text {-synuclein that re- } \\
\text { moves the stop codon and introduces Not I } \\
\text { and Nhel sites downstream }\end{array}$ & $\begin{array}{l}\text { ggggctagcgcggccgccac- } \\
\text { cGGCTTCAGGTTCGTAG- } \\
\text { TCTTG }\end{array}$ & $56^{\circ} \mathrm{C}$ \\
\hline oGQ1723 & $\begin{array}{l}\text { fwd primer for eat-3 cDNA for N-terminal } \\
\text { fusions that introduces Eco47III, Notl and } \\
\text { Agel sites upstream of start ATG }\end{array}$ & $\begin{array}{l}\text { cccagcgctggcggccgcggaccgg- } \\
\text { tagccATGCGGATCGCCACAA- } \\
\text { GACGCCGATT }\end{array}$ & $68^{\circ} \mathrm{C}$ \\
\hline
\end{tabular}




\begin{tabular}{|c|c|c|c|}
\hline oGQ1724 & $\begin{array}{l}\text { rev primer for eat-3 cDNA for N-terminal fu- } \\
\text { sions that introduces Xhol and Smal sites } \\
\text { downstream of the stop codon }\end{array}$ & $\begin{array}{l}\text { ccccccgggctcgagTCAGAT- } \\
\text { TTTTTCACGTTGTAATTGAGC }\end{array}$ & $57^{\circ} \mathrm{C}$ \\
\hline oGQ1725 & $\begin{array}{l}\text { fwd Primer for fis-1 cDNA for N-terminal fu- } \\
\text { sions that introduces Eco47III, Notl and Agel } \\
\text { sites upstream of start ATG }\end{array}$ & $\begin{array}{l}\text { cccagcgctggcggccgcggaccgg- } \\
\text { tagccATGGAAC- } \\
\text { CTGAATCGATTCTCGA }\end{array}$ & $57^{\circ} \mathrm{C}$ \\
\hline oGQ1726 & $\begin{array}{l}\text { rev primer for fis-1 cDNA for N-terminal fu- } \\
\text { sions that introduces Xhol and Smal sites } \\
\text { downstream of the stop codon }\end{array}$ & $\begin{array}{l}\text { cccccgggctcgag- } \\
\text { TCATTTCCTGAAA- } \\
\text { GCAAGTCCGG }\end{array}$ & $59^{\circ} \mathrm{C}$ \\
\hline oGQ1727 & $\begin{array}{l}\text { fwd primer for fzo-1 cDNA for N-terminal } \\
\text { fusions that introduces Eco } 47 \text { III and Notl } \\
\text { sites upstream of start ATG (Agel cuts inside } \\
\text { gene) }\end{array}$ & $\begin{array}{l}\text { cccagcgctggcggccgcggaccgg- } \\
\text { tagccATGTCTGGCACAGCAA- } \\
\text { GCTTAGTTC }\end{array}$ & $61^{\circ} \mathrm{C}$ \\
\hline oGQ1728 & $\begin{array}{l}\text { rev primer for fzo-1 cDNA for N-terminal fu- } \\
\text { sions that introduces a Smal sites down- } \\
\text { stream of the stop codon (Xhol and EcoRI } \\
\text { cut inside gene): }\end{array}$ & $\begin{array}{l}\text { ccccccgggctcgag- } \\
\text { TCATGGCGTTGGCGGAGAG- } \\
\text { TCC }\end{array}$ & $65^{\circ} \mathrm{C}$ \\
\hline oGQ1948 & $\begin{array}{l}\text { forward primer to amplify the catp- } 6 \text { promoter } \\
4 \text { kb fragment that introduces a Pstl site up- } \\
\text { stream }\end{array}$ & gggctgcagtgatcattgcggtcgtctgga & $60^{\circ} \mathrm{C}$ \\
\hline oGQ1949 & $\begin{array}{l}\text { reverse primer to amplify the catp-6 promoter } \\
\text { that start just upstream of the start ATG and } \\
\text { introduces a BamHI and Kpnl site }\end{array}$ & $\begin{array}{l}\text { gggggtaccg- } \\
\text { gatcctcggtggcgtcgtgcgccacc }\end{array}$ & $79^{\circ} \mathrm{C}$ \\
\hline oGQ1358 & $\begin{array}{l}\text { Forward primer } 1 \text { for detecting the eri-1 } \\
\text { (mg366) insertion }\end{array}$ & actgatgggtaaggaatcgaagacg & $60^{\circ} \mathrm{C}$ \\
\hline oGQ1359 & $\begin{array}{l}\text { Reverse primer } 1 \text { for detecting the eri-1 } \\
\text { (mg366) insertion }\end{array}$ & gataaaacttcggaacatatggggc & $58^{\circ} \mathrm{C}$ \\
\hline oGQ1849 & $\begin{array}{l}\text { Deletion Primer for catp-6 (ok3473) outer left } \\
\text { Sequence: }\end{array}$ & cttaaaatttcgcggctgag & $53^{\circ} \mathrm{C}$ \\
\hline oGQ1852 & $\begin{array}{l}\text { Deletion Primer for catp-6 (ok3473) inner } \\
\text { right sequence: }\end{array}$ & tggtgagctcgatgaatacg & $55^{\circ} \mathrm{C}$ \\
\hline oGQ2221 & $\begin{array}{l}\text { Fwd Primer (external) catp-6 for screening for } \\
\text { tm3190 }\end{array}$ & ccagctcttccaggtaacct & $57^{\circ} \mathrm{C}$ \\
\hline oGQ2222 & $\begin{array}{l}\text { Primer (external) catp-6 for screening for } \\
\text { tm3190 deletion }\end{array}$ & ggttgaacattatcgaatcgttgag & $56^{\circ} \mathrm{C}$ \\
\hline oGQ1906 & $\begin{array}{l}\text { Fwd primer for gfp w/o stop that introduces a } \\
\text { Bglll and Nhel }\end{array}$ & $\begin{array}{l}\text { cccagatctgctagcATGAG- } \\
\text { TAAAGGAGAAGAACTT }\end{array}$ & $65^{\circ} \mathrm{C}$ \\
\hline oGQ1907 & $\begin{array}{l}\text { rev Primer for gfp w/o stop that introduces a } \\
\text { Nhel and Agel }\end{array}$ & $\begin{array}{l}\text { ccctaccggtccgctagcGCCAATT- } \\
\text { GGAGTATTTTGTTG }\end{array}$ & $71^{\circ} \mathrm{C}$ \\
\hline
\end{tabular}


Table 8.4: List of injection mixes used in this study.

\begin{tabular}{|c|c|c|}
\hline $\begin{array}{l}\text { Injection mix } \\
\text { name }\end{array}$ & Plasmids of interest & Markers \\
\hline InNo17 & $40 \mathrm{ng} / \mu \mathrm{L}$ pmyo-3::wt $\alpha \mathrm{S}-m Y F P$ & $\begin{array}{l}40 \mathrm{ng} / \mu \mathrm{L} \text { pRF4 (rol-6(su1006)) } \\
20 \mathrm{ng} / \mu \mathrm{L} \text { pttx-3::gfp }\end{array}$ \\
\hline InNo23 & $40 \mathrm{ng} / \mu \mathrm{L}$ pmyo-3::TP $\alpha \mathrm{S}-m Y F P$ & $\begin{array}{l}40 \mathrm{ng} / \mu \mathrm{L} \text { pRF4 (rol-6(su1006)) } \\
20 \mathrm{ng} / \mu \mathrm{L} \text { pttx-3::gfp }\end{array}$ \\
\hline InNo29 & $\begin{array}{l}5 \mathrm{ng} / \mu \mathrm{L} \text { pmyo-3::tagrfp-drp-1 } \\
5 \mathrm{ng} / \mu \mathrm{L} \text { pmyo-3::tom70-cfp }\end{array}$ & $\begin{array}{l}40 \mathrm{ng} / \mu \mathrm{L} \text { pRF4 (rol-6(su1006)) } \\
20 \mathrm{ng} / \mu \mathrm{L} \text { pttx-3::gfp }\end{array}$ \\
\hline InNo30 & $\begin{array}{c}5 \mathrm{ng} / \mu \mathrm{L} \text { pmyo-3::tom70-cfp } \\
55 \mathrm{ng} / \mu \mathrm{L} \text { pmyo-3::wt } \alpha S-m Y F P\end{array}$ & $40 \mathrm{ng} / \mu \mathrm{L}$ pRF4 (rol-6(su1006)) \\
\hline InNo31 & $5 \mathrm{ng} / \mu \mathrm{L}$ pmyo-3::tom70-cfp & $40 \mathrm{ng} / \mu \mathrm{L}$ pRF4 (rol-6(su1006)) \\
\hline InNo33 & $\begin{array}{c}5 \mathrm{ng} / \mu \mathrm{L} \text { pmyo-3::tom70-cfp } \\
30 \mathrm{ng} / \mu \mathrm{L} \text { pmy-o3::wt } \alpha S-m Y F P\end{array}$ & $40 \mathrm{ng} / \mu \mathrm{L}$ pRF4 (rol-6(su1006)) \\
\hline InNo39 & $\begin{array}{c}5 \mathrm{ng} / \mu \mathrm{L} \text { pmyo-3::tom70-cfp } \\
30 \mathrm{ng} / \mu \mathrm{L} \text { pmyo-3::TP } \alpha S-m Y F P\end{array}$ & $40 \mathrm{ng} / \mu \mathrm{L}$ pRF4 (rol-6(su1006)) \\
\hline InNo41 & $\begin{array}{l}5 \mathrm{ng} / \mu \mathrm{L} \text { pmyo-3::tom70-cfp } \\
5 \mathrm{ng} / \mu \mathrm{L} \text { pmyo-3::wt } \alpha S-m Y F P\end{array}$ & $40 \mathrm{ng} / \mu \mathrm{L}$ pRF4 (rol-6(su1006)) \\
\hline InNo42 & $5 \mathrm{ng} / \mu \mathrm{L}$ prab-3::tom70-cfp & $40 \mathrm{ng} / \mu \mathrm{L}$ pRF4 (rol-6(su1006)) \\
\hline InNo43 & $\begin{array}{c}5 \mathrm{ng} / \mu \mathrm{L} \text { prab-3::tom70-cfp } \\
30 \mathrm{ng} / \mu \mathrm{L} \text { prab-3::wt } \alpha S-m Y F P\end{array}$ & $40 \mathrm{ng} / \mu \mathrm{L}$ pRF4 (rol-6(su1006)) \\
\hline InNo47 & $\begin{array}{c}5 \mathrm{ng} / \mu \mathrm{L} \text { pmyo-3::tom70-cfp } \\
30 \mathrm{ng} / \mu \mathrm{L} \text { pmyo-3::A30P } \alpha S-m Y F P\end{array}$ & $40 \mathrm{ng} / \mu \mathrm{L}$ pRF4 (rol-6(su1006)) \\
\hline InNo50 & $\begin{array}{l}5 \mathrm{ng} / \mu \mathrm{L} \text { pmyo-3::tom70-cfp } \\
30 \mathrm{ng} / \mu \mathrm{L} \text { pmyo-3::A53T aS-mYFP }\end{array}$ & $40 \mathrm{ng} / \mu \mathrm{L}$ pRF4 (rol-6(su1006)) \\
\hline InNo61 & $\begin{array}{l}5 \mathrm{ng} / \mu \mathrm{L} \text { prab-3::tom70-cfp } \\
5 \mathrm{ng} / \mu \mathrm{L} \text { prab-3::tagrfp-drp-1 }\end{array}$ & $40 \mathrm{ng} / \mu \mathrm{L}$ pRF4 (rol-6(su1006)) \\
\hline InNo62 & $\begin{array}{l}5 \mathrm{ng} / \mu \mathrm{L} \text { pmyo-3::tagrfp-drp-1 } \\
5 \mathrm{ng} / \mu \mathrm{L} \text { pmyo-3::tom70-cfp }\end{array}$ & $40 \mathrm{ng} / \mu \mathrm{L}$ pRF4 (rol-6(su1006)) \\
\hline InNo68 & $5 \mathrm{ng} / \mu \mathrm{L}$ pmyo-3::yfp-catp-6 & $5 \mathrm{ng} / \mu \mathrm{L}$ pmyo-2::gfp \\
\hline InNo70 & $40 \mathrm{ng} / \mu \mathrm{L}$ pcatp-6::gfp & $30 \mathrm{ng} / \mu \mathrm{L}$ pRF4 (rol-6(su1006)) \\
\hline InNo71 & $80 \mathrm{ng} / \mu \mathrm{L}$ pcatp-6::gfp & $30 \mathrm{ng} / \mu \mathrm{L}$ pRF4 (rol-6(su1006)) \\
\hline
\end{tabular}

Table 8.5: List of strains used in this study.

\begin{tabular}{|c|c|c|}
\hline $\begin{array}{l}\text { Strain } \\
\text { name }\end{array}$ & Genotype & Injection mix/Line \\
\hline GQ155 & gzEx117 [pmyo-3::tom70-cfp; rol-6(su1006)] & $\ln N o 31 / 2$ \\
\hline GQ156 & gzEx118 [pmyo-3::tom70-cfp; rol-6(su1006)] & InNo31/9 \\
\hline GQ157 & $\begin{array}{l}\text { gzEx119 [pmyo-3::wt aS-myfp; pmyo-3::tom70-cfp; } \\
\text { rol-6(su1006)] }\end{array}$ & $\ln N 033 / 3$ \\
\hline GQ158 & $\begin{array}{l}\text { gzEx120 [pmyo-3::wt aS-myfp; pmyo-3::tom70-cfp; } \\
\text { rol-6(su1006)] }\end{array}$ & $\ln N o 33 / 4$ \\
\hline GQ159 & $\begin{array}{l}\text { gzEx121 [pmyo-3::wt aS-myfp; pmyo-3::tom70-cfp; } \\
\text { rol-6(su1006)] }\end{array}$ & $\ln N o 30 / 3$ \\
\hline GQ160 & gzEx122 [prab-3::tom70-cfp; rol-6(su1006)] & InNo42/1 \\
\hline GQ161 & gzEx123 [prab-3::tom-70-cfp; rol-6(su1006)] & InNo42/4 \\
\hline GQ162 & $\begin{array}{l}\text { gzEx124 [prab-3:: wt aS-myfp; prab-3::tom70-CFP; } \\
\text { rol-6(su1006sd)] }\end{array}$ & $\ln N 043 / 1$ \\
\hline GQ163 & $\begin{array}{l}\text { gzEx125 [prab-3:: wt aS-myfp; prab-3::tom70-CFP; } \\
\text { rol-6(su1006sd)] }\end{array}$ & $\ln N 043 / 2$ \\
\hline GQ164 & $\begin{array}{l}\text { gzEx126 [prab-3:: wt aS-myfp; prab-3::tom70-CFP; } \\
\text { rol-6(su1006sd)] }\end{array}$ & $\ln N 043 / 3$ \\
\hline GQ165 & $\begin{array}{l}\text { gzEx127 [pmyo-3::wt aS-myfp; pmyo-3::tom70-cfp; } \\
\text { rol-6(su1006)] }\end{array}$ & $\operatorname{InNo30/1}$ \\
\hline GQ166 & $\begin{array}{l}\text { gzEx128 [pmyo-3::wt aS-myfp; pmyo-3::tom70-cfp; } \\
\text { rol-6(su1006)] }\end{array}$ & $\ln N o 30 / 2$ \\
\hline GQ167 & $\begin{array}{l}\text { gzEx129 [pmyo-3::wt aS-myfp; pmyo-3::tom70-cfp; } \\
\text { rol-6(su1006)] }\end{array}$ & $\ln N 033 / 1$ \\
\hline
\end{tabular}




\begin{tabular}{|c|c|c|}
\hline CB5600 & him-8; ccls4251 [pmyo-3::Ngfp-lacZ; pmyo-3::Mtgfp] & \\
\hline GQ707 & catp-6(ok3473) 2* & \\
\hline GQ708 & catp-6(tm3190) $2^{*}$ & \\
\hline GQ709 & N2; gzEx214[pcatp-6::gfp] & InNo70/1 \\
\hline GQ710 & N2; gzEx215 [pcatp-6::gfp] & InNo71/1 \\
\hline GQ186 & $\begin{array}{l}\text { catp-6(ok3473) gzEx212 [pmyo-3::tom70-cfp; } \\
\text { pmyo-3::tagrfp-drp-1; rol-6(su1006)] }\end{array}$ & $\ln N o 29 / 3$ \\
\hline GQ670 & $\begin{array}{l}\text { gzEx212 [pmyo-3::tom70-cfp;tagrfp-drp-1; } \\
\text { rol-6(su1006)] }\end{array}$ & $\ln N o 29 / 3$ \\
\hline GQ671 & $\begin{array}{l}\text { eri-1(mg366); gzEx212 [pmyo-3::tom70-cfp; } \\
\text { pmyo-3::tagrfp-drp-1; rol-6(su1006)] }\end{array}$ & $\ln N 029 / 3$ \\
\hline GQ711 & $\begin{array}{l}\text { catp-6(ok3473) eri-1(mg366); } \\
\text { gzEx212[pmyo-3::tom70-cfp; pmyo-3::tagrfp-drp-1; } \\
\text { rol-6(su1006)] }\end{array}$ & InNo29/3 \\
\hline PD4595 & ccls4595 [ceh-24::gfp; rol-6(su1006)] & \\
\hline GQ712 & catp-6(ok3473); ccls4595 [ceh-24::gfp; rol-6(su1006)] & \\
\hline SJ4100 & $z c / s 13[h s p-6:: g f p]$ & \\
\hline GQ713 & catp-6(ok3473) ; zcls13 [hsp-6::gfp] & \\
\hline SJ4005 & $z c / s 4$ [hsp-4::gfp] & \\
\hline GQ714 & catp-6(ok3473) ; zcls4[hsp-4::gfp] & \\
\hline CL2070 & dvls70 [hsp-16.2::gfp; rol-6(su1006)] & \\
\hline GQ715 & catp-6(ok3473); dvls70 [hsp-16.2::gfp; rol-6(su1006)] & \\
\hline SS104 & $g / p-4(b n 2)$ & \\
\hline GQ716 & catp-6(ok3473); glp-4(bn2) & \\
\hline QG717 & catp-6(tm3190); glp-4(bn2) I & \\
\hline GQ718 & $\begin{array}{l}\text { N2; gzEx216[pmyo-3::catp-6; pmyo-3::tom70-cfp; } \\
\text { pmyo-3::tagrfp_t-drp-1] }\end{array}$ & InNo62-2 \\
\hline GQ719 & $\begin{array}{l}\text { catp-6(ok3473); gzEx216[pmyo-3::catp-6; } \\
\text { pmyo-3::tom70-cfp;p myo-3::tagrfp_t-drp-1] }\end{array}$ & InNo62-2 \\
\hline GQ720 & $\begin{array}{l}\text { N2; gzEx217[prab-3::tom70-cfp; } \\
\text { prab-3::tagrfp_t-drp-1] }\end{array}$ & InNo61-8 \\
\hline GQ721 & $\begin{array}{l}\text { catp-6(ok3473); gzEx217[prab-3::tom70-cfp; } \\
\text { prab-3::tagrfp_t-drp-1] }\end{array}$ & InNo61-8 \\
\hline MD701 & bcls39[plim-7::ced-1-gfp] & \\
\hline GQ722 & catp-6(ok3473); bcls39[plim-7::ced-1-gfp] & \\
\hline DA2123 & adls2122[lgg-1::GFP, rol-6(su1006)] & \\
\hline GQ723 & catp-6(ok3473); adls2122[lgg-1::GFP, rol-6(su1006)] & \\
\hline RW1596 & stEx30[pmyo-3::myo-3p::GFP, rol-6(su1006)] & \\
\hline GQ724 & $\begin{array}{l}\text { catp-6(ok3473);stEx30[pmyo-3::myo-3::GFP, } \\
\text { rol-6(su1006)] }\end{array}$ & \\
\hline CF1553 & muls84[psod-3::gfp] & \\
\hline GQ725 & catp-6(ok3473); muls84[psod-3::gfp] & \\
\hline GQ726 & catp-6(tm3190); muls84[psod-3::gfp] & \\
\hline GQ727 & N2; gzEx218[pmyo-3::yfp-catp-6; pmyo-2::gfp] & InNo/68-1 \\
\hline GQ728 & $\begin{array}{l}\text { catp-6(ok3473); gzEx218[pmyo-3::yfp-catp-6; } \\
\text { pmyo-2::gfp] }\end{array}$ & $\mathrm{InNo} / 68-1$ \\
\hline
\end{tabular}




\subsection{Media, solutions and buffers}

\author{
Luria-Bertani (LB) Medium, liquid \\ $5 \mathrm{~g}$ Peptone \\ $2.5 \mathrm{~g}$ Yeast extract \\ $5 \mathrm{~g} \mathrm{NaCl}$ \\ Add $\mathrm{ddH}_{2} \mathrm{O}$ to $1 \mathrm{~L}$ \\ $\rightarrow$ Autoclave
}

Luria-Bertani (LB) Medium, for plates

$5 \mathrm{~g}$ Peptone

$2.5 \mathrm{~g}$ Yeast extract

$5 \mathrm{~g} \mathrm{NaCl}$

$7.5 \mathrm{~g} \quad$ Agar

Add $\mathrm{ddH}_{2} \mathrm{O}$ to $1 \mathrm{~L}$

$\rightarrow$ Autoclave

If needed add Amp (1:500) after cooling down to

$60^{\circ} \mathrm{C}$ to achieve a final concentration of $100 \mu \mathrm{g} / \mathrm{mL}$

\section{M9 buffer}

$3 \mathrm{~g} \quad \mathrm{KH} 2 \mathrm{PO} 4$

$6 \mathrm{~g} \quad \mathrm{Na} 2 \mathrm{HPO} 4$

$5 \mathrm{~g} \quad \mathrm{NaCl}$

Add $\mathrm{ddH}_{2} \mathrm{O}$ to $1 \mathrm{~L}$

$\rightarrow$ Autoclave

Worm lysis/PCR buffer (10x)

$1 \mathrm{M}$ Tris $\mathrm{pH} 8.3$

$5 \mathrm{M} \mathrm{KCl}$

$200 \mathrm{mM} \mathrm{MgCl}_{2}$

\section{1x TBST}

$10 \mathrm{mM}$ Tris- $\mathrm{HCl} \mathrm{pH} 8.0$

$0.05 \%$ Tween 20

$150 \mathrm{mM} \mathrm{NaCl}$

\section{SDS PAGE Running buffer(10x)}

2x PAGE sample buffer with

$\beta$-Mercaptoethanol

$121.1 \mathrm{~g} / \mathrm{L}$ Tris $\mathrm{pH} 6.8$

$20 \% \quad$ Glycerol (v/v)

$100 \mathrm{~g} / \mathrm{L}$ SDS

$0.002 \% \quad$ Bromophenol blue (w/v)

$200 \mathrm{~mL} \quad \beta$-Mercaptoethanol (Sigma Aldrich)

Add $\mathrm{ddH}_{2} \mathrm{O}$ to $1 \mathrm{~L}$

\section{Blotting buffer \\ $5.8 \mathrm{~g} \quad$ Tris \\ $2.9 \mathrm{~g} \quad$ Glycine \\ $0.37 \mathrm{~g}$ SDS \\ $400 \mathrm{~mL} \quad \mathrm{MeOH}$ \\ Add $\mathrm{ddH}_{2} \mathrm{O}$ to $1 \mathrm{~L}$ \\ Adjust to $\mathrm{pH} 8.9$}

$30.3 \mathrm{~g}$ Tris

$144 \mathrm{~g}$ Glycine

$10 \mathrm{~g}$ SDS

Add $\mathrm{dd}_{2} \mathrm{O}$ to $1 \mathrm{~L}$

Adjust to $\mathrm{pH} 8.3$

\section{Stripping buffer}

$\begin{array}{ll}100 \mathrm{mM} & \beta \text {-Mercaptoethanol } \\ 2 \% & \text { SDS } \\ 62.5 \mathrm{mM} & \text { TRIS-HCl } \\ 400 \mathrm{~mL} & \mathrm{MeOH} \\ \text { Add ddH } \mathrm{H}_{2} 0 \text { to } 1 \mathrm{~L} & \\ \text { Adjust to } \mathrm{pH} 6.7 & \end{array}$




\section{Curriculum vitae}

\section{NORA WENDER}

European Neuroscience Institute

Grisebachstr. 5, 37077 Göttingen

Phone: +49-(0)551-39 13901

Email: nwender@gwdg.de

\section{EdUCATION}

2008 - 2012 PhD Student, University of Göttingen, Germany

European Neuroscience Institute, Department for Molecular Neurogenetics

(Dr. Stefan Eimer)

Cellular function and toxicity of Parkinson's disease-related genes in C. elegans

2007 - 2012 Master-/PhD-Program Neurosciences International Max Planck Research School, University of Göttingen, Germany

2004 - 2007 Bachelor of Science in Molecular Biotechnology University of Heidelberg; Germany

2004

Abitur (German general qualification for university entrance) Ludwigsgymnasium, Saarbrücken, Germany

AWARDS/HONORS/FELLOWSHIPS

2010 - 2011 PhD-Fellowship from the German National Academic Foundation ("Studienstiftung des deutschen Volkes")

2010 - 2012 PhD-Stipend from the Dorothea-Schlözer-Program of the University of Göttingen

2005 - 2009 Stipend from the German National Academic Foundation

2004 Awarded for the best exam in chemistry and best "Abitur" of the class of 2004 Ludwigsgymnasium, Saarbrücken, Germany

2003 1. Prize at the youth research competition "Jugend forscht", Saarland, Germany

Project: „Non-destructive measurement method to determine the sintering state of ceramics"

\section{Professional EXPERIENCE}

2011 Co-Organizer of the student-organized conference "Neurizons - From Molecules to Mind: Making Sense of the Brain", Göttingen, Germany

2009-2011 Supervision of three graduate students (2-months lab rotation each) Teaching Assistance in tutorials and methods courses about $C$. elegans for Master students in Neuroscience

2007 Internship at Boehringer-Ingelheim, Biberach, Germany, Department for Parkinson's Disease Research (Prof. Bastian Hengerer)

2005 - 2007 Student Research Assistant, Max-Planck-Institute for Medical Research, Heidelberg, Germany, Department of Cell Physiology (Prof. Bert Sakmann) 


\section{PATENTS}

2004 German Patent for a method to determine the sinter state of ceramics (together with Dr. Andreas Hoffmann und Lukas Wallrich)

\section{Conferences}

$2010 \quad 1^{\text {th }}$ International C. elegans Meeting, Los Angeles, USA, Poster: "Cellular toxicity of different alpha-synuclein variants in C. elegans"

2011 Conference "Intracellular trafficking processes", Rehovot, Israel, Talk: "Cellular toxicity of different alpha-synuclein variants in C. elegans"

2010 International PhD Symposium "Horizons in Molecular Biology", Göttingen, Germany, Poster: "Cellular toxicity of different alpha-synuclein variants in C. elegans"

$2010 \quad$ International PhD Symposium "Protein Trafficking in Health and Disease", Hamburg, Germany, Poster: "Cellular toxicity of different alphasynuclein variants in $C$. elegans"

2009 International PhD Symposium "Horizons in Molecular Biology", Göttingen, Germany, Poster: „The Parkinson's Disease-associated Kinases Pink-1 and Lrk-1 act antagonistically in C. elegans"

$2009 \quad$ ENInet (Network of European Neuroscience Institutes) Meeting and ENInet PhD-Symposium, Crete, Greece Poster: „The Parkinson's Diseaseassociated Kinases Pink-1 and Lrk-1 act antagonistically in C. elegans" Neurizons-Conference in Göttingen, Poster: „The Parkinson's Diseaseassociated Kinases Pink-1 and Lrk-1 act antagonistically in C. elegans"

\section{VOLUNTEER EXPERIENCE}

$2006-2008$ Board member of "juFORUM e.V." (Association of Young Researchers in Germany)

2007 Member of the Committee responsible for dispensing the tuition fees at the Institute for Pharmacy and Molecular Biotechnology at the University of Heidelberg

2004 - 2007 Member of the Student Council in the Department for Molecular Biotechnology at the University of Heidelberg

LANGUAGES German: native speaker, English: fluent (TOEFL iBT 112/120 points), French: fluent (DALF), Spanish: intermediate 


\section{List of publications}

Kamp F, Exner N, Lutz AK, Wender N, Hegermann J, Brunner B, Nuscher B, Bartels T, Giese A, Beyer K, Eimer S, Winklhofer KF, Haass C. (2010) Inhibition of mitochondrial fusion by $\alpha$-synuclein is rescued by PINK1, Parkin, and DJ-1. EMBO J 29(20):3571-89.

Karpinar DP, Balia MB, Kügler S, Opazo F, Rezaei-Ghaleh N, Wender N, Kim HY, Taschenberger G, Falkenburger BH, Heise H, Kumar A, Riedel D, Fichtner L, Voigt A, Braus GH, Giller K, Becker S, Herzig A, Baldus M, Jäckle H, Eimer S, Schulz JB, Griesinger C, Zweckstetter M. (2009) Prefibrillar alpha-synuclein variants with impaired beta-structure increase neurotoxicity in Parkinson's disease models. EMBO J 28(20):3256-68. 